Florida International University FIU Digital Commons

\title{
Creation and Evaluation of Polymer/Multiwall Carbon Nanotube Films for Structural Vibration Control and Strain Sensing Properties
}

weiwei lin

wlin005@fiu.edu

DOI: $10.25148 /$ etd.FIDC001208

Follow this and additional works at: https://digitalcommons.fiu.edu/etd

Part of the Nanoscience and Nanotechnology Commons, Other Materials Science and Engineering Commons, Polymer and Organic Materials Commons, and the Structural Materials Commons

\section{Recommended Citation}

lin, weiwei, "Creation and Evaluation of Polymer/Multiwall Carbon Nanotube Films for Structural Vibration Control and Strain Sensing Properties" (2016). FIU Electronic Theses and Dissertations. 3025.

https://digitalcommons.fiu.edu/etd/3025 


\section{FLORIDA INTERNATIONAL UNIVERSITY}

Miami, Florida

CREATION AND EVALUATION OF POLYMER/MULTIWALL CARBON NANOTUBE FILMS FOR STRUCTURAL VIBRATION CONTROL AND STRAIN SENSING PROPERTIES

A dissertation submitted in partial fulfillment of the requirements for the degree of DOCTOR OF PHILOSOPHY

in

MATERIALS SCIENCE AND ENGINEERING

by

Weiwei Lin 
To: Interim Dean Ranu Jung

College of Engineering and Computing

This dissertation, written by Weiwei Lin, and entitled Creation and Evaluation of Polymer/Multiwall Carbon Nanotube Films for Structural Vibration Control and Strain Sensing Properties, having been approved in respect to style and intellectual content, is referred to you for judgement.

We have read this dissertation and recommend that it be approved.

Arvind Agarwal

Benjamin Boesl

Kang K. Yen

Xiaohua Li

Cesar Levy, Major Professor

Date of Defense: November 10, 2016

The dissertation of Weiwei Lin is approved.

Interim Dean Ranu Jung

College of Engineering and Computing

Andres G. Gil

Vice President for Research and Economic Development and Dean of the University Graduate School

Florida International University, 2016 


\section{DEDICATION}

I dedicate this dissertation to my family: my parents, Wanneng LIN and Caijun Tian ; and my husband, Yue YU, without whose support and endless love, the completion of this work would not have been possible. Also, I dedicate this dissertation to both of my lovely sons, whom I love with all my life. 


\section{ACKNOWLEDGMENTS}

First of all, I want to express my deepest appreciation to my major professor, Dr. Cesar Levy, for his great guidance, continuous encouragement and financial support to me to finish this project. I would also like to thank Florida International University's Graduate Fellowship Committee for the Dissertation Year Fellowship funding that allowed me to write this dissertation. Also, I want to thank the members of my committee, Dr. Arvind Agarwal, Dr. Benjamin Boesl, Dr. Kang K. Yen and Dr. Xiaohua Li for their guidance and help in my work.

I would also like to thank Dr. Xiangxing Kong, Dr. Weiyu Bao and Dr. Brian Reding for their helpful suggestions and support by providing the instruments used for the experimental testing; Dr. Yanqing Liu for his help on the TEM, SEM and XRD images; Mr. Andy Nieto and Dr. Pranjal Nautiyal for their help on the nanoindentation tests; and Mr. Richard Zicarelli for his help in creation of the molds used to form the sensor samples.

I would like to thank my colleagues and friends for the numerous times they helped me out with my work. They are Dr. Hadi Fekrmandi, Mr. Yonatan Rotenberg, and Mr. Kevin Ward. 


\begin{abstract}
OF THE DISSERTATION
CREATION AND EVALUATION OF POLYMER/MULTIWALL CARBON

NANOTUBE FILMS FOR STRUCTURAL VIBRATION CONTROL AND STRAIN

SENSING PROPERTIES
\end{abstract}

by

Weiwei Lin

Florida International University, 2016

Miami, Florida

Professor Cesar Levy, Major Professor

Multifunctional materials both with damping properties and strain sensing properties are very important. They promise to be more weight-efficient, and provide volume-efficient performance, flexibility and potentially, less maintenance than traditional multi-component brass-board systems.

The goal of this dissertation work was to design, synthesize, investigate and apply polyaniline/Multiwall carbon nanotube (PANI/MWCNT) and polyurethane (PU) /MWCNT composites films for structural vibration control and strain sensors using free layer damping methods and static and dynamic strain sensing test methods.

The PANI/MWCNT was made by in situ polymerization of PANI in the presence of MWCNT, then frit compression was used to make circular and rectangular PANI/MWCNT composite films. PU/MWCNT composites were made by the layer-bylayer method. Free end vibration test results showed both of PANI/MWCNT and PU/MWCNT have better damping ratios than each of their components. Static sensing 
test indicated that though there appears to be residual strain in both composite sensors after the load is removed, both the sensor and the foil strain gage react linearly when re-engaged. A drift test of the sensor showed that it is stable. The dynamic sensing test results showed that over the range of $10-1000 \mathrm{~Hz}$, the PANI/MWCNT composite sensor was consistently superior to foil strain gage for sensing purposes since the highest peak consistently corresponded to the input frequency and was much higher, for example, at $20 \mathrm{~Hz}, 820$ times higher than those of the strain gage. Using the same criterion, the PU/Buckypaper composite sensor was comparable to or superior to the foil strain gage for sensing purposes over the range of $10 \mathrm{~Hz}$ to $200 \mathrm{~Hz}$.

The relationship of loss factor, $\eta$, and beam coverage length, $\mathrm{L} 1 / \mathrm{L}$, is discussed for single sided and double sided attachment. For both PANI/MWCNT and PU/MWCNT, the loss factor, $\eta$, was found to increase as coverage length, $\mathrm{L} 1 / \mathrm{L}$, increases. The loss factor, $\eta$, was found to have a maximum as with coverage length, $\mathrm{L} 1 / \mathrm{L}$, as the coverage length continues to increase. The trend for double sided attachment was found to follow the trends discussed by Rao (2004) and Levy and Chen (1994) for viscoelastic material constrained damping. 


\section{TABLES OF CONTENTS}

CHAPTER

PAGE

I. INTRODUCTION........................................................

1.1 Multifunctional Materials with Damping and Strain Sensing Properties.......1

1.2 Multifunctional Materials of Polymer/CNT composites......................2

1.3 Limitation of Current Research......................................... 4

1.4 Objective of Present Study............................................. 8

1.5 The Dissertation Outline................................................. 8

II. LITERATURE REVIEW ..............................................9

2.1 Multifunctional Materials................................................ 9

2.2 Structural Damping and Vibration Control................................12

2.2.1 Passive Structure Vibration and Noise Control......................12

2.2.2 Active and Hybrid Structure Vibration Control....................... 16

2.2.3 Active Vibration Control Using Strain Sensors......................18

2.3 Strain Sensors Research Background...................................20

2.4 Background of Multifunctional Materials Having Both Structural

Damping Properties and Strain Sensing Capabilities........................23

2.5 Polyaniline/MWCNT and Polyurethane/MWCNT .........................25

2.5.1 Polyaniline/MWCNT ............................................25

2.5.2 Polyurethane/MWCNT .........................................27

2.6 Novelty of The Present Work.......................................... 30

III. PREPARATION OF CIRCULAR POLYANILINE/MULTIWALLED

CARBON NANOTUBE AND CHARACTERIZATION OF THEIR

STRUCTURAL DAMPING AND SRTAIN SENSING PROPERTIES ..........31

3.1 Introduction .......................................................... 31

3.2 Experimental Sections............................................. 31

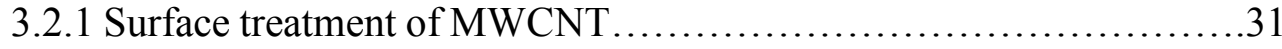

3.2.2 In situ synthesizing of circular PANI/11\% MWCNT composites......33

3.2.3 Characterization................................................. 35

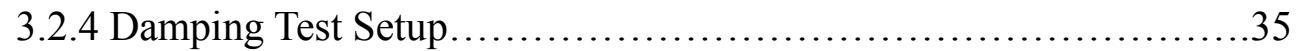

3.2.5 The Strain Sensing Experiment Setup.............................37

3.3 Results and Discussion............................................. 38

3.3.1 Electrical Conductivity of the PANI/MWCNT Film................. 38

3.3.2 Mechanical Properties of the PANI/MWNT Nanocomposite Films...39

3.3.3 Thermal Stability of the PANI/MWCNT Nanocomposites Films......40

3.3.4 Damping Ratios Discussion..................................42

3.3.5 Strain Sensor Properties of PANI/11\% MWCNT Nanocomposites...43

3.3.5.1 Theory of Strain Sensor..................................43

3.3.5.2 The Drift Test of PANI/MWCNT Sensor.....................45

3.3.5.3 The Linearity of the PANI/MWCNT .........................46 
IV. PREPARATION OF RECTANGULAR POLYANILINE/MULTIWALLED CARBON NANOTUBE AND CHARACTERIZATION OF THEIR STRUCTURAL DAMPING AND STRAIN SENSING PROPERTIES.........53

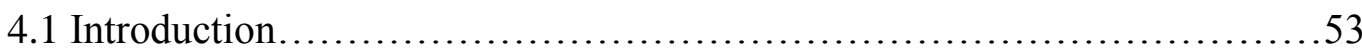

4.2 Experimental Sections............................................5 53

4.2.1 In Situ Synthesizing of Rectangular PANI/MWCNT Composites......53

4.2.2 Characterization...............................................56

4.2.3 Specimen Setup for Damping Test Purpose........................56

4.2.4 Damping Test Methodology......................................61

4.2.5 Specimen Setup for Strain Sensing Test Purpose...................61

4.2.5.1 Comparison of Different Components of PANI/MWCNT.....61

4.2.5.2 Sample Setup for Drift, Static Tensile Test for the PANI $/ 11 \%$ MWCNT................................. 62

4.2.5.3 Drift Test Procedures for the PANI/11\% MWCNT Sensor.......63

4.2.5.4 Static Test Procedures for the PANI/11\% MWCNT Sensor......63

4.2.6 Results and Discussion........................................64

4.2.6.1 Morphological Characterization of the PANI/

$22 \%$ MWCNT Film........................................64

4.2.6.2 Damping Ratios Comparison of Different Component PANI/MWCNT ......................................66

4.2.6.3 Comparison Single Side and Both Sides' Attachment of PANI/MWCNT ........................................68

4.2.6.4 The Thickness Effect of Aluminum Base Beam.................69

4.2.6.5 Damping Ratios of Different Compression Force of PANI/MWCNT ....................................... 70

4.2.6.6 Damping Results for Single Side Attachment...................71

4.2.6.7 Damping Results for Single Side Attachment..................73

4.2.7 Strain Sensing Properties of PANI/MWCNT ...........................75

4.2.7.1 Comparison of Different Components of PANI/MWCNT......75

4.2.7.2 The Repeatability of Strain Sensing Property of

PANI/ MWCNT Composites.................................76

4.2.7.3 Dynamic Sensing Capability of the PANI/MWCNT...........78

4.2.7.4 The Drift Test of PANI/MWCNT Sensor.................... 81

4.2.7.5 Linearity and Repeatability of PANI/MWCNT Sensor........ 84

4.3 Conclusions

V. PREPARATION OF POLYURETHANE/MULTIWALLED CARBON NANOTUBE AND CHARACTERIZATION OF THEIR STRUCTURAL DAMPING AND SRTAIN SENSING PROPERTIES

5.1 Introduction. 
5.2 Experimental Sections

5.2.1 Mold Casting Method to Make Pu/MWCNT/ZnO

Nanowires Composites .92

5.2.1.1 The procedure of Fabrication $\mathrm{ZnO}$ nanowires.................92

5.2.1.2 Dry Heat Treatment of MWCNT ..............................95

5.2.1.3 Fabrication of PU/MWCNT, PU/ZnO and

$\mathrm{PU} / \mathrm{MWCNT} / \mathrm{ZnO}$ nanowires films.........................95

5.2.1.4 Production of PU/DMF Solution............................96

5.2.2 Binding Layer Method to Make

PU/MWCNT/ZnO Nanorods/Nanowires Films......................97

5.2.3 Oriented Method to Make PU/MWCNT/ZnO

Nanorods/Nanowires Films.................................... 98

5.2.4 Problems Encountered with Those Methods........................ 99

5.2.4.1 Disadvantages of Mold Casting method....................... 99

5.2.4.2 Disadvantages of Binding Layer and Oriented Methods......100

5.2.5 Layer by Layer Attachment Methods to Prepare Pu/Buckypaper... 101

5.2.6 Sample Setup for Free Vibration Tests........................... 102

5.2.6.1 Single Side Attachment Mode..............................102

5.2.4.2 Both Sided Attachment Mode.............................. 104

5.2.7 Damping Test Methodology................................ 105

5.2.8 Sample Setup for Drift, Static Tensile Test, and Dynamic Tests....105

5.2.9 Drift Test Procedures.........................................106

5.2.10 Static Test Procedures.........................................106

5.2.11 Dynamic Test Procedures.........................................107

5.3 Results and discussion..........................................107

5.3.1 Damping Ratios of PU, Buckypaper and PU/Buckypaper..........107

5.3.2 Damping Results for Single Side Attachment......................109

5.3.3 Damping Results for Single Side Attachment....................110

5.3.4 Static Sensing Properties.................................... 113

5.3.4.1 The Drift Effect..........................................113

5.3.4.2 Linearity and Repeatability - Static Tensile Test.............. 115

5.3.5 Dynamic Sensing Characteristic..............................118

5.4 Conclusion.......................................................... 122

VI. DISCUSSION OF YOUNG'S MODULUS ................................. 125

VII. CONCLUSION AND FUTURE WORK ............................ 130

7.1 The Multifunctional PANI/MWCNT Composites Films..................130

7.2 The Multifunctional PU/Buckypaper Films............................ 132

$7.3 \mathrm{PU} / \mathrm{ZnO} / \mathrm{MWCNT}$ composites.......................................... 132

7.4 Future Research................................................ 133

REFERENCES........................................................ 134

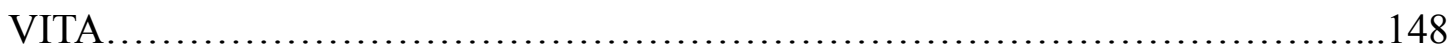




\section{LIST OF TABLES}

TABLE

PAGE

Table 2.1 Loss coefficient of materials (Rao, 2004) .14

Table 2.2 Damping ratios of different types of construction/ arrangement. (Rao, 2004) .16

Table 3.1 Different components of nanocomposites films .34

Table 3.2 Damping ratio values of PANI/MWCNT films in different components and different aluminum beam dimensions.

Table 3.3 Damping ratios of PANI/11\% MWCNT fixed on either the \# or * sides

Table 3.4 The comparison of PANI/22\% MWCNT and PANI/44\% MWCNT when covered by a very thin layer of glued to shield from the environment and when not covered by the glue.

Table 4.1 Synthesizing different components of PANI/MWCNT composites and its control.

Table 4.2 Different thickness and distance from the clamp and the samples were tested.

Table 4.3 Raw data strain sensing test comparison of different components of PANI/MWCNT composite films.

Table 4.4 Trend line slopes for Figure 4.26. .83

Table 4.5 Slopes and intercepts for the PANI/MWCNT sensor given in Figure 4.28.

Table 5.1 Conditions of synthesizing $\mathrm{ZnO}$ nanorods/nanowires

Table 5.2 Diameters, length and aspect ratio of nano-ZnO in different synthesizing conditions....

Table 5.3 Trend line slopes and R-values 


\section{LIST OF FIGURES}

FIGURE

PAGE

Figure 1.1 (a) Reliable contact and seal treatments of MWCNT film.................4

(b) test coupon in the MTS machine..................................

(c) Linear and repeatable relationship between the MWCNT resistance and the applied strain and ..................................

(d) MWCNT resistance vs. temperature ( $\mathrm{Li}$ et al., 2008)..................4

Figure 2.1 Damping treatment. (a) FLD treatments and......................... 15

(b) PCLD treatments (Renninger, 2004.)............................15

Figure 2.2 Representation of a piezoelectric capacitance in parallel with shunt impedance. piezoelectric patch represented as an equivalent capacitance (Trindade and Maio, 2008).

Figure 2.3 Schematic representation of the cantilever sandwich beam with three piezoelectric patches (not to scale) (Trindade and Maio, 2008). .............18

Figure 2.4 The experimental setup of the measurement system (Ma et al., 2011)......20

Figure 2.5 (a) The location of the PVDF sensor and the impact location on the top surface of the cantilever beam................................20

(b) The location of the strain gauge on the bottom surface of the cantilever beam (Ma et al., 2011)....

Figure 2.6 Experimental setup for testing the proposed nanotubebased sensors (Ramaratnam and Jalili, 2006)

Figure 3.1 The dry heat treatment processing of MWCNT. 32

Figure 3.2 (left) A frit compression system for making nanocomposites films.........34

(a) syringe column; (b) plungers; (c) polypropylene frits and................34

(d) composites suspension ....................................... 34

(right) Photo images of PANI/MWNT nanocomposites dics.......................34

Figure 3.3 Digital pictures of attached samples on different aluminum beams with dimension by (a) 5.13 inch $\times 1$ inch $\times 0.12$ inch, and...........36

(b) 2.4 inch $\times 1$ inch $\times 0.008$ inch.................................

Figure 3.4 Dimension of samples, aluminum beams, the locations where samples were held fixed. (a) and (b) $6 \mathrm{~cm}$ long beam (c) $9.3 \mathrm{~cm}$ long.......37 beam with fixed ends $0.8 \mathrm{~cm}$ from the left-hand side or $0.6 \mathrm{~cm} \ldots \ldots \ldots \ldots . . . . .37$ from the right-hand side; and (d) $12.6 \mathrm{~cm}$ long aluminum beams............37 with fixed ends $0.8 \mathrm{~cm}$ from the left-hand side or $0.9 \mathrm{~cm}$ from the...........37 right hand side. Red lines were the locations sample side \# and...............37 free end side * where the beam is held fixed..............................37 
Figure 3.5 Strain sensor test setup.

Figure 3.6 Electrical conductivity and standard deviations of PANI/11\% MWCNT,

PANI $/ 22 \%$ MWCNT, PANI/44\% MWCNT, pure PANI and

pure MWCNT films measured by multimeter.

Figure 3.7 Nanoindentation (loading-unloading curves) of pristine PANI, pure

MWCNT and PANI $/ 22 \%$ MWCNT nanocomposite film....

Figure 3.8 SDT curve for pristine MWCNT, pure PANI and

PANI $/ 22 \%$ MWCNT composites films.

Figure 3.9 Drift test comparison of pure PANI, PANI/11\% MWCNT,

PANI $/ 22 \%$ MWCNT, PANI $/ 44 \%$ MWCNT and strain gauge sensors..... . .46

(a) series 1 is pure PANI, (b) series 2 is PANI/11\% MWCNT.............. 46

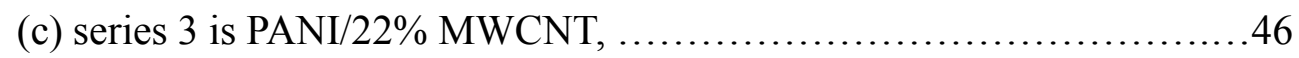

(d) series 4 is PANI/44\% MWCNT and............................... 46

(f) series 5 is traditional foil strain gauge.............................46

Figure 3.10 Strain sensor test of PANI/22\% MWCNT showing load vs. output........47

Figure 3.11 Strain sensor test of PANI/22\% MWCNT with glue showing load vs. output.

Figure 3.12 Strain sensor test of PANI/44\% MWCNT without glue covered showing load vs. output.....

Figure 3.13 Strain sensor test of PANI/44\% MWCNT with glue showing load vs. output. .48

Figure 3.14 TEM images of (a) pure MWCNT and (b) PANI/11\% MWCNT .49

Figure 3.15 SEM image of (a) pure MWCNT, (b) pure PANI and ....................50

(c) PANI $/ 1 \%$ MWCNT composite films. .50

Figure 4.1 The frit compression system for making PANI/MWCNT nanocomposite films 55

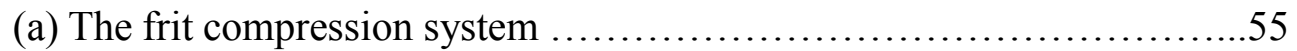

(b) are the photo image of PANI/MWCNT nanocomposites..............55

Inserts are A: cuboid; B: plungers; C: polypropylene frits and..............55

D: composites suspension.

Figure 4.2 Schematic image of $289 \mathrm{~N}$ and $334 \mathrm{~N}$ force applied to plungers measured by FlexiForce adapter. The adapter's load measurements have a $\pm 3 \%$ error.

Figure 4.3 (a) Size of samples, Al beams and the locations where samples were attached.

(b) Locations of sample side \# and free end side * 
where the beam was clamped.

Figure 4.4 (a) PANI/11\% MWCNT sandwiched Al base beam

(b) PANI $/ 11 \%$ MWCNT attached one side of Al base beam.

Figure 4.5 Dimension of samples and locations (red lines) where the clips clamped on (a) $0 \mathrm{~cm}$, (b) $1.7 \mathrm{~cm}$, (c) $5.4 \mathrm{~cm}$ and (d) $7.7 \mathrm{~cm}$ away to the samples.....59 The dimension of the sample is $4 \mathrm{~cm} \times 0.8 \mathrm{~cm} \times 1.9 \mathrm{~mm} \ldots \ldots \ldots \ldots \ldots \ldots . .59$

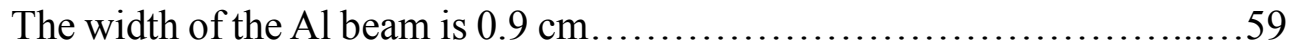

Figure 4.6 Schematic images of single side's attachment. The dimension of samples, $\mathrm{Al}$ beams and the locations where samples were attached, the locations of sample side \# and free end side * where the beam was clamped and also the directions and lengths for both cutting and clamping....

Figure 4.7 Schematic images for sandwich attachment. The dimension of samples, $\mathrm{Al}$ beams and the locations where samples were attached, the locations of sample side \# and free end side * where the beam was clamped and also the directions and lengths for both cutting and clamping.............60

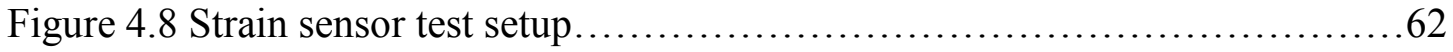

Figure 4.9 SEM image of (a) pure MWCNT, (b) pure PANI and ...................65

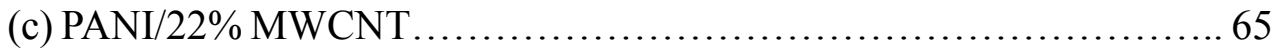

Figure 4.10 TEM images of (a) pure MWCNT and.............................66

(b) PANI $/ 22 \%$ MWCNT nanocomposite films.......................66

(a'), (b') and (c') were higher magnification $(\times 10,000) \ldots \ldots \ldots \ldots \ldots \ldots . \ldots 6$

images of (a), (b) and (c), respectively..........................66

Figure 4.11 Damping ratios and standard deviation of rectangular pure PANI,

PANI $/ 11 \%$ MWCNT, PANI/22\% MWCNT, PANI/44\% MWCNT and pure MWCNT clamped on the uncovered $*$ side and sample \# side.

Figure 4.12 Comparison of free vibration damping test between

PANI $/ 11 \%$ MWCNT single side covered (red) and PANI/11\% MWCNT

double sides covered (black) Al base beam..........................68

Figure 4.13 Damping ratios of $2.4 \mathrm{~mm}$ and $1.25 \mathrm{~mm}$ thickness $\mathrm{Al}$ beams with different distance from the clamped side of the beam to the right end of the sample

Figure 4.14 Damping ratio comparison between $289 \pm 3 \% \mathrm{~N}$ and $334 \pm 3 \% \mathrm{~N}$ compression force of PANI/MWCNT when clamped on the free end side. Error bars are provided for each test....

Figure 4.15 Damping ratios of single side attached PANI/MWCNT clamped on the sample side and on the free end side.

Error bars are provided for each test. 
Figure 4.16 Damping ratios of both sides attached PANI/MWCNT clamped on the free end side and on the sample side.

Error bars are provided for each test. ...

Figure 4.17 The relationship of loss factor $(\eta)$ and beam coverage L1/L between single side and double sided attachment of the PANI/MWCNT sample for both clamping conditions.

Figure 4.18 Strain sensor test comparison of different components

of PANI/MWCNT.

Figure 4.19 (a) The linearity and repeatability of strain sensor test of PANI $/ 11 \%$ MWCNT, PANI/22\% MWCNT and PANI $/ 44 \%$ MWCNT, showing load vs. output

Figure 4.19 (b) The linearity and repeatability of strain sensor test of PANI $/ 11 \%$ MWCNT, PANI $/ 22 \%$ MWCNT and PANI $/ 44 \%$ MWCNT, showing load vs. output

Figure 4.19 (c) The linearity and repeatability of strain sensor test of PANI $/ 11 \%$ MWCNT, PANI/22\% MWCNT and PANI $/ 44 \%$ MWCNT, showing load vs. output .77

Figure 4.20 Beam and sensor in dynamic testing mode. Beam and sensor being interrogated by the laser vibrometer. .78

Figure 4.21 Frequency response functions (FRF) of the PANI/MWCNT sensor and foil strain gage for frequency range 10-200 Hz.

Figure 4.22 Frequency response functions of the PANI/MWCNT sensor and foil strain gage for $400-1000 \mathrm{~Hz}$

Figure 4.23 Superposition of the frequency response functions of the PANI/MWCNT sensor and foil strain gage for low and high frequency. Graphs represent a superposition of the previous graphs to indicate the superior sensitivity of the PANI/MWCNT in the $10-1000 \mathrm{~Hz}$ range and the $1000-5000 \mathrm{~Hz}$ range

Figure 4.24 Wheatstone bridge setup for load sensing.

Figure 4.25 Same beam with strain gage showing and with sensor showing, both in loading mode

Figure 4.26 PANI/MWCNT sensor and strain gage drift test. Drift trend lines for typical PANI/MWCNT sensor and strain gage. Vertical scale is in $\mathrm{mV}$; horizontal scale is in minutes

Figure 4.27 Drift test for PANI/MWCNT (sensor 3) and drift test for strain gage......84 Figure 4.28 Voltage-strain curves for the PANI/MWCNT sensor. Note initial 
linearity of the sensor then marked change in slope in which there is an apparent step, then linear once again at much lower slope

Figure 4.29 Voltage- strain curve for strain gage. The strain gage shows linear voltage-strain behavior until unload and returns to the same start value.....85

Figure 5.1 TEM image of the hydrothermal synthesized $\mathrm{ZnO}$ at..................99

(a) $160{ }^{\circ} \mathrm{C}$, (b) $180{ }^{\circ} \mathrm{C}$, (c) $200{ }^{\circ} \mathrm{C}$ for $20 \mathrm{~h}$ and........................... 94

(d) a typical SAED pattern of a single $\mathrm{ZnO}$ nanowire....................94

Figure 5.2 TEM images of the hydrothermal synthesized $\mathrm{ZnO}$ at

(a) $160{ }^{\circ} \mathrm{C}$ and (b) $200{ }^{\circ} \mathrm{C}$ for $20 \mathrm{~h}$

Figure 5.3 Procedures of making PU/MWCNT/ZnO nanorods/nanowires composites films

Figure 5.4 Schematic diagram of make PU/MWCNT/ZnO nanorods composites films

Figure 5.5 Schematic diagram of make PU/MWCNT/ZnO nanorods composites films

Figure 5.6 Schematic image of PU/MWCNT/ZnO nanorods/nanowires composites films attached upon Al base beam with non-conductive adhesive.

Figure 5.7 The process for preparation of PU/Buckypaper.

Figure 5.8 Schematic images of single side attachment. The dimension of samples, Al beams and the locations where samples were attached, the locations (red line) where the beam was clamped and the directions and lengths for beam removal....

Figure 5.9 Schematic images of single side's attachment. The dimension of samples, Al beams and the locations where samples were attached, the locations of sample side \# and free end side * where the beam was clamped and the directions and lengths for both beam removal and clamping.

Figure 5.10 Schematic images of double sides' attachment. The dimension of samples, Al beams and the locations where samples were attached, the locations of sample side \# and free end side * where the beam was clamped and the directions and lengths for both beam removals and clamping....

Figure 5.11 Damping ratios comparison of PU, Buckypaper and PU/Buckypaper composites. Error bars are shown for each sample at each beam length....108

Figure 5.12 Damping ratios of single side attached PU/Buckypaper clamped on the free end $*$ side and the sample \# side. Error bars are shown for each result shown... 
Figure 5.13 Damping ratios of double sides attached PU/Buckypaper clamped on the uncovered $*$ side and sample \# side.

Error bars are shown for each result shown.

Figure 5.14 Comparison between single side and double sided attachment of The PU/Buckypaper sample for both clamping conditions....

Figure 5.15 (a) Drift trendlines for typical PU/Buckypaper sensor and strain gage...114

Figure 5.15 (b) Drift test for PU/Buckypaper sample (sensor 5) and.

(c) Drift test for strain gage.

Figure 5.16 The repeat loading comparison of PU/Buckypaper and foil strain gage-the raw data.

Figure 5.17 The repeat loading comparison of PU/Buckypaper and foil strain gage-data linearity.

Figure 5.18 The loading/unloading comparison of PU/Buckypaper versus strain--first load (gray)/unload (red) and second load (yellow)/ unload (blue) of the sensor.

Figure 5.19 Frequency response comparison of the strain gages and the PU/Buckypaper.

Figure 5.20 Frequency response comparison of the strain gages and the PU/Buckypaper.

Figure 5.21 Frequency response comparison of the strain gages and the PU/Buckypaper.

Figure 5.22 Superposition of the frequency response functions of the PU/ Buckypaper sensor and foil strain gage in the $10-400 \mathrm{~Hz}$ range.

Figure 6.1 Nanoindentation (loading-unloading curves) of PANI/11\%

MWCNT sensor.

Figure 6.2 Nanoindentation (loading-unloading curves) of PANI/22\%

MWCNT sensor

Figure 6.3 Tension test method (load-displacement) result for PANI/11\% MWCNT. .128

Figure 6.4 Tension test ( $1^{\text {st }}$ and $2^{\text {nd }}$ load-displacement) result for PANI/11\% MWCNT. 


\section{LIST OF SYMBOLS}

b

E

$\mathrm{F}_{\mathrm{o}}$

G

$\mathrm{H}$

$\mathrm{h}$

L

$\mathrm{L}_{1}$

$\Delta \mathrm{L}$

I

P

$\mathrm{R}$

$\Delta \mathrm{R}$

V

$\mathrm{V}_{\mathrm{s}}$

$\mathrm{V}_{\mathrm{o}}$

W

$\Delta \mathrm{W}$

$\mathrm{X}$

$\eta$

$\varepsilon$
The width of the beam

Young's modulus

Amplitude

The gauge factor

Hardness

The thickness of the beam

The length from the support to the free end of the beam

The distance from the gauge to the load

The change in length of the beam

The moment of inertia of the beam

Applied load at the tip of the beam

Electrical resistance

The change in resistance of the gauge

Voltage output

The source voltages

The output voltages

Maximum strain energy in cycle

Energy dissipated during 1 cycle of harmonic displacement

Displacement

Loss factor (or loss coefficient)

The strain on the upper surface of the beam 


\section{$\underline{\text { ABBREVIATIONS }}$}

APS

$\mathrm{Al}$

AFM

BMA

Buckypaper

CNT

CVD

CLD

DMF

DMA

EMI

FLD

FRF

FMWCNT

$\mathrm{HNO}_{3}$

$\mathrm{HCl}$

$\mathrm{H}_{2} \mathrm{SO}_{4}$

MWCNT

MMA

$\mathrm{NiTi}$

$\left(\mathrm{NH}_{4}\right)_{2} \mathrm{CO}_{3}$
Ammonium persulphate

Aluminum

Atomic force microscopy

Butyl methacrylate

Multi-wall carbon nanotubes films

Carbon nanotubes

Chemical vapor deposition

Passive constrained layer damping

Dimethylfuoride

Dynamic mechanical analysis

Electromagnetic interference

Free layer damping

Frequency response functions

Functionalized multi-wall carbon nanotubes

Nitric acid

Hydrochloric acid

Sulfuric acid

Multi-wall carbon nanotubes

Methyl methacrylate

Nickel titanium

Ammonium carbonate 
PU

PANI

PDMS

PPL

PCLD

PEM

PVDF

$\mathrm{P}(\mathrm{VDF}-\mathrm{TrFE})$

SWCNT

SMA

SEM

SDT

SAED

$\mathrm{TiO}_{2}$

TPU

TDI

TEM

VEM

WPC

$\mathrm{ZnO}$

$\mathrm{Zn}\left(\mathrm{NO}_{3}\right)_{2} \cdot 6 \mathrm{H}_{2} \mathrm{O}$
Polyurethane

Polyaniline

Polydimethylsiloxane

Polymer Pen Lithography

Passive constrained layer damping

Piezoelectric materials

Polyvinylidene fluoride

Poly (vinylidene fluoride-trifluoroethylene)

Single-wall carbon nanotubes

Shape memory materials

Scanning electron microscopy

Simultaneous DSC-TGA

Selected area electron diffraction

Titanium dioxide

Thermoplastic polyurethanes

Toluene diisocyanate

Transmission electron microscopy

Viscoelastic materials

Wood polymer composites

Zinc oxide

Zinc Nitrate Hexahydrate 6 DiHydrogen Monoxide 


\section{CHAPTER I}

\section{INTRODUCTION}

\subsection{Multifunctional Materials with Damping and Strain Sensing Properties}

Multifunctional materials were designed to improve overall system performance, which has sought to integrate at least one other function into a material that is capable of, for example, bearing mechanical loads and serves as a structural material element. Because with conventional structural materials, it has been difficult to achieve simultaneous improvement in multiple structural functions. Multifunctional materials that can be attained are electrical/thermal conductivity, sensing and actuation, electromagnetic interference (EMI) shielding, recyclability and biodegradability, et al.

Structural-borne vibration and noise control is one of the key issues associated with the application of structures and machines. Consequently, the demand of materials with high structural damping capacity is growing in a variety of sectors (Koratkar et al., 2002; Suhr et al., 2005; Suhr et al., 2006). Multifunctional materials, both with damping properties and strain sensing properties are very important. They promise more weightefficient, volume-efficient performance, flexibility and potentially, less maintenance than traditional multi-component brass-board systems, which have attracted many researchers' interests recently. For example, it is beneficial and crucial to retrofit critical infrastructures, including aircraft, ships, highways, bridges, buildings, and pipelines, both with sensors that have damping and strain sensing properties. This is important because these infrastructures are sometimes subjected to severe loading conditions due to hurricanes, earthquakes and other natural disasters during their lifetime. 
Multifunctional materials having damping properties can sense and prevent or at least minimize catastrophic failures and subsequent loss of life, while strain sensing property materials provide further inspection tools that can evaluate the problems caused by these types of disasters. Also, the structural-borne vibration is one of the key issues for army combat vehicles, gun platforms and the gun barrels. Successful vibration suppression improves the accuracy of surveillance, targeting and firing.

\subsection{Multifunctional Materials of Polymer/CNT composites}

Carbon nanotubes (CNT) have many excellent properties which have fascinated scientists for decades. Particularly, their extraordinary mechanical properties and electrical conductivities have been studied widely. CNT are the ideal structural component candidates, for they are the strongest and stiffest materials yet discovered in terms of tensile strength and elastic modulus respectively. Researches have shown that nanocomposites containing single-wall or multi-wall carbon nanotubes (SWCNT or MWCNT) can achieve high damping capacity by taking advantage of the weak bonding and interfacial friction between individual nanotubes and the matrix (Sasikumar et al., 2012; Liu et al., 2012; Gong et al., 2012; Agrawal et al., 2013; Tehrani et al., 2013). Also, the unique electrical conductivities of CNT make them very popular in producing conductive nanocomposites. For example, due to the piezoresistivity property of CNT themselves, pure MWCNT films (bulky paper) were found that serve as strain sensors if they were bonded to the structural surface as conventional foil strain gauges (Li and Levy, 2007a; Hong et al., 2012). Furthermore. the new type of 
electrically conductive $\mathrm{CNT} /$ polymer nanocomposites have been broadly studied to apply to piezoresistive or resistance-type strain sensors (Alamusi et al., 2013; Qu et al., 2014; Costa et al., 2014).

Koratkar research group (2002) has demonstrated that a thin layer of MWCNT deposited by chemical vapor deposition method (CVD) and sandwiched between two silicon substrates can dramatically increase the damping ratio and stiffness of the sandwich structure simultaneously. After that, many papers, for example, Y. Li et al. (2015), Alva and Raja (2014), Alnefaie et al. (2013), Tehrani et al. (2013), Hazarika and Maji (2014) and Xiong et al. (2006) were published in which the damping index of polymer composites was increased when CNT were introduced. Theoretical analysis also showed that the addition of CNT into a polymer matrix can improve the damping ratio and stiffness of the matrix (Buffa et al., 2007).

Li and Levy's preliminary research results (2007a) have indicated that Buckypaper (MWCNT films) sandwiched with two electrical non-conductive adhesives are capable of being strain sensors. Also, free vibration test of this new type of carbon nanotubes based composite showed some small damping properties. In a word, the electrical nonconductive adhesive sandwiched buckypaper was potentially capable of functioning simultaneously as a strain sensor and damping treatment for structural vibration control but needed a larger damping component.

Hazarika and Maji (2013) used Raman spectroscopy to study the relationship between MWCNT and wood polymer composites (WPC). They found that there was a linear relationship (with a negative slope) between the $\mathrm{G}^{\prime}$-band shift and the applied 
strain for the WPC samples loaded with MWCNT. They noticed that the higher the amount of MWCNT, the more efficient was the load transfer from wood/polymer to MWCNT. Also, they found an improvement in elastic modulus, loss modulus and damping index when adding MWCNT in WPC indicated by the dynamic mechanical analysis (DMA) test.

$\mathrm{Cu}$ layer by thermal deposition

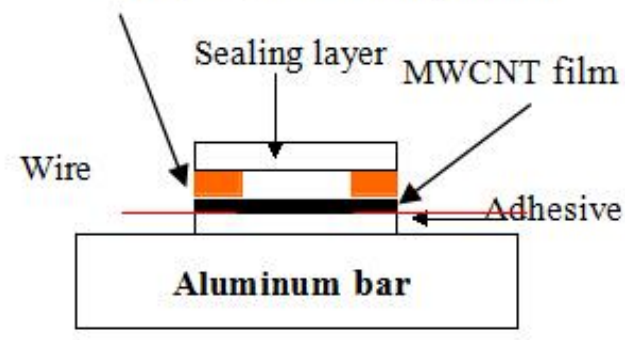

(a)

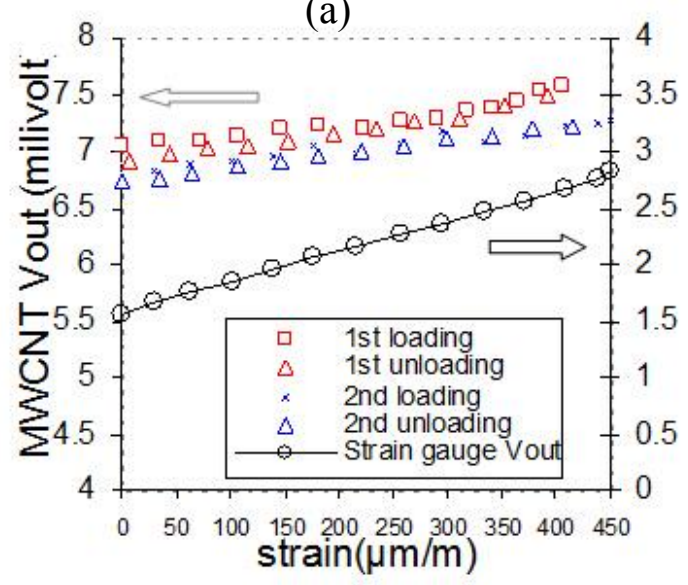

(c)

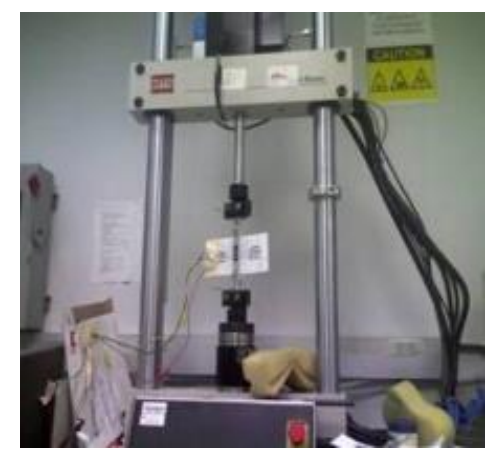

(b)

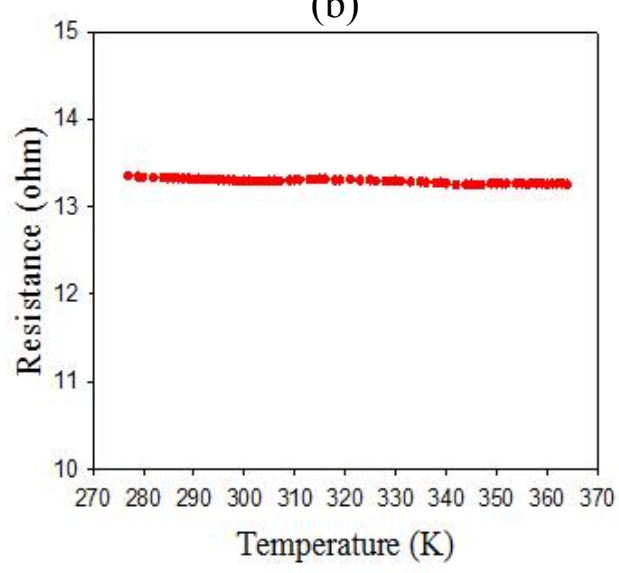

(d)

Figure 1.1 (a) Reliable contact and seal treatments of MWCNT film; (b) Test coupon in the MTS machine. (c) Linear and repeatable relationship between the MWCNT resistance and the applied strain and (d) MWCNT resistance vs. temperature (Li et al., 2008).

\subsection{Limitation of Current Research}

Vibration control usually applies viscoelastic materials (VEM) together with a constraining layer bonded to the original structure making up a sandwich structure 
(Kerwin, 1959). The most commonly used viscoelastic materials for vibration control are polyurethanes (PU), such as the DYAD 601 polymer from Soundcoat Inc. The main advantages of using VEM as damping layer includes low cost, safety, easy installation and no need of external power. However, the conventional VEM such as polyurethane are frequency and temperature-dependent materials, thus optimal damping is restricted in a narrow range of frequencies and temperatures (Johnson, 1995). Besides, the VEM layers must be thick enough for tough structures to achieve satisfactory damping, which leads to the addition of considerable weight to the original structures. In order to reduce the weight added to the original structure, partially covered sandwich beams and plates were studied by Lall (1987), Levy and Chen (1994), et al. The applications of constraining VEM treatment for cylindrical shell structures were also investigated by El-Raheb and Wagner (1986), Lall et al. (1987) and Ramesh and Ganesan (1994).

Typical sensors for structural vibration control such as optical fiber sensors, accelerometers, foil strain gauges and piezoelectric ceramics/polymers are not multifunctional and need extra effort of installation. The large number (usually hundreds and thousands) of sensors required for application usually led to higher financial cost, complicated network and sophisticated data analysis. We want to create a sensor which is simple, effective, economic, which owning multifunctional properties for passive/active vibration and noise control.

Although our preliminary research results showed the electrical non-conductive adhesive sandwiched buckypaper acted like a strain sensor, CNT are too expensive. 1 $\mathrm{g}$ of 5-20 $\mu \mathrm{m}$ length and diameter about $15 \pm 5 \mathrm{~nm}$ costs $\$ 110$ (Nanolab). Furthermore, 
there is limited knowledge about the change of either chemical or physical properties when adding NanoSperse AQ surfactant into MWCNT when making buckypaper, a step which is very essential for the creation of buckypaper. It's for this reason that research of polymer/MWCNT composite is being undertaken.

Multifunctional materials are necessarily composite materials, and the strong growth in the use of composites has been greatly influenced by multifunctional design requirements. The most widely used composites have polymer matrix materials, which are typically poor conductors. Enhanced conductivity could be achieved with reinforcing the composite with $\mathrm{CNT}$ for instance. Carbon nanotubes have attracted much attention since their discovery in 1991 due to their unique mechanical and electronic properties (Iijima, 1991). Addition of carbon nanotube enhances the properties of polymer even at very low concentrations due to their large aspect ratio and ultra-high conductivity (Sobha and Narayanankutty, 2015). And these carbon nanotube based polymer composites have potential uses in many field, such as strain sensors, damping augmentation, super capacitors, electromagnetic radiation shield coatings, actuators etc (Xu and Allen, 2013; Ansari et al., 2014; Sivakkumar et al., 2007; Saini et al., 2009; Mottaghitalab et al., 2006).

However, despite many reports claiming successful preparation, controlling the microstructure of such a composite system remains a challenge. This is primarily due to the strong interaction forces that cause the formations of bundles and clusters in asproduced CNT. Conventional methods of producing a composite involves directly mixing CNT into a polymer matrix. The dispersion and arrangement of CNT in the 
matrix hold a central role in controlling the properties of the resulting composites. Hence, there is a need to develop the new type of CNT involved multifunctional materials both with damping and strain sensing properties. And the way how to disperse the CNT into the polymer matrix should investigated.

Polyaniline (PANI) is one of the most studied conducting polymers of the past 50 years, and it is the cheapest one among its conducting polymer family. Up to now, most PANI/CNT nanocomposites materials obtained by the chemical methods were in the powder form (Huang et al., 2010). Though some papers stated that binding materials were used when making PANI/CNT composites (Qiao et al., 2007; Wang et al., 2009; Lu et al., 2011), they were thought to change the mechanical or chemical properties of PANI/CNT composites films. Flexible carbon nanotube/polyaniline films were fabricated by immersing the MWCNT network template into the in situ aniline polymerization solution (Lu et al., 2011). However, their electrical, electrochemical or mechanical properties could not be mostly optimized due to their heterogeneous nature. Furthermore, due to its difficulty to control the thickness of PANI/MWCNT nanocomposites films, there is few papers reporting their damping properties.

We are investigating the creation of an inexpensive polymer/MWCNT composite that can act both as a strain sensor as well as the damping material for the purpose of structural vibration control. However, due to the large aspect ratio of the CNT, the dispersion and directional alignment of the MWCNT in the polymer matrix creation of such a sensor is still a great challenge. 


\subsection{Objective of Present Study}

The objective of the present work consisted of the creation of sensor-actuators which addressed two main issues, namely: the ability to sense strain in a linear and repeatable fashion; and, the ability to attenuate vibrations that were sensed. These tasks were investigated through the determination of the damping properties and the strain sensing properties-including sensing repeatability-of the sensors that were created and evaluated, namely sensor-actuators made of PANI/MWCNT and PU/MWCNT films. Furthermore, the best locations of these sensors were investigated as a means of optimizing the vibration attenuation. These sensors were created simply and inexpensively and with minimal weight addition. The objective was achieved by: (1) Synthesizing of PANI/MWCNT composites and PU/MWCNT composites by different methods; (2) Use of free end vibration methods to characterize their damping properties; (3) and use of static and dynamic strain sensing tests to evaluate these composites.

\subsection{The Dissertation Outline}

Chapter II provides a detailed literature reviews on multifunctional materials both as structural damping materials and strain sensors; the details of structural damping and vibration control and the PANI, MWCNT, PU and their composites as strain sensing and damping material. Chapter III and Chapter IV characterized the circular and rectangular PANI/MWCNT of damping and strain sensing properties. Damping and strain sensing properties of PU/MWCNT were described in Chapter V. Chapter VI discussed the Young's modulus of the PANI/MWCNT. Chapter VII summarized the important conclusions and recommendations for future research. 


\section{CHAPTER II}

\section{LITERATURE REVIEW}

2.1 Multifunctional Materials

By contrast to conventional materials, which are difficult to achieve simultaneous improvement in multiple functions, multifunctional materials are designed to meet specific requirements through tailored properties to improve the overall system performance. Multifunctional materials can have many functions vary from mechanical to electrical and thermal functions, which promise more weight-efficient, volume-efficient performance flexibility and potentially less maintenance than traditional multicomponent brass-board systems. For examples, multifunctional materials can be attained are electrical/thermal conductivity (Liu et al., 2013; Liu et al., 2014), energy harvesting/storage ( Anton et al., 2010; Xu et al., 2015; Xie et al., 2015), self-healing capability (Zheng et al., 2016; Liu et al., 2016), EMI shielding (Luo et al., 2016; Mondal et al., 2016; Fazita et al., 2015) and recyclability and biodegradability (Fazita et al., 2015; Nakamura et al., 2004), etc. A multifunctional material is typically a composite or hybrid of several distinct material phases, which required a new design methodology in which system-level performance is emphasized over the optimization of individual functions. Also, composite materials are ideally suited to achieve multifunctionality since the best features of different materials can be combined to form a material that has a broad spectrum of desired properties.

The polymer composites can be designed to have multifunctional functions by modifying their surface (Xie et al., 2016). For examples, Feng's research group (2012) 
created polyethylene superhydrophobic surface by thermal micro-molding method with a thin layer titanium dioxide $\left(\mathrm{TiO}_{2}\right)$ nanoparticles coated upon it. This designed polyethylene surface could attain the superhydrophobic capability and can act like lotus leaves, which are even too vulnerable to be used in daily life. Also, they innovatively created polymer superhydrophobic surface by the thermal pressing method with the assistance of polydimethylsiloxane (PDMS) as a soft template. The attained polymer surfaces are such that in micro/nano second-order structure showed superhydrophobic properties. In other words, additional functions are obtained, such as superhydrophobic properties, by only giving certain structures to polymer surface (Feng, 2009). Also, Polymer Pen Lithography (PPL) was fabricated by polymerization of methyl methacrylate (MMA) with butyl methacrylate (BMA) with various ratios. The obtained PPL showed much more printing ability than the traditional printing materials PDMS, which can be replaced by PPL (Zhong et al., 2013). Morphology into cross-linked polymer network was achieved by photoinduced cross-linking of reactive monomers within highly evaporative solutions in the presence of water vaper. The resulting films show superior resistance against solvent dissolution, enhanced thermal stability and water repellence (Wang et al., 2012).

Carbon nanotube can lend new functionality to polymer by enhancing their strength and their thermal conductivity and electrical conductivity. CNT-based composites have been world widely studied because they have the potential to revolutionize structural materials for aerospace, electrical and thermal conductors for energy applications, nano-biotechnology, and other disciplines. However, though CNT typically have very 
high aspect ratios, their absolute lengths remain short. Also, along with the difficulty in dispersion in polymer matrix, interfacial stress transfer problems, the demand on the alignment and even the high cost of CNT, which prevent us from developing composite structures that could supplement or replace like the conventional aerospace materials. However, there are a number of research efforts underway that address these and other concerns and achievement was attained (Balani et al., 2007; Suzuki et al., 2016; Almkhelfe et al., 2016). For example, Wang research group (2013) fabricated the novel CNT composites with the long length, high volume fraction, good alignment and reduced waviness of the CNT, which have been demonstrated to have unprecedented multifunctionalities, including record high strength $(3.8 \mathrm{GPa})$, high Young's modulus $(293 \mathrm{GPa})$, electrical conductivity $\left(1230 \mathrm{~S} \cdot \mathrm{cm}^{-1}\right)$ and thermal conductivity $\left(41 \mathrm{Wm}^{-1}\right.$ $\left.\mathrm{K}^{-1}\right)$.

The multifunctional materials with both structural vibration damping properties and strain sensing properties are one of the most importance both in civil infrastructures and military use. Because for civil infrastructures, such as aircraft, ships, highways, bridges, buildings, pipelines, are sometimes subjected to severe loading conditions due to hurricanes, earthquakes and other natural disasters during their lifetime. Multifunctional materials having damping properties can sense and prevent or at least minimize catastrophic failures and subsequent loss of life, while strain sensing property materials provide further inspection tools that can evaluate the problems caused by these types of disasters. 


\subsection{Structural Damping and Vibration Control}

Structural vibration and noise control is one of great interest and importance in the worldwide engineering fields. Damping is the energy dissipation properties of a material or system under cyclical stress. Its mechanism is the conversion of mechanical energy into thermal energy. Vibration damping materials is used to reduce or eliminate noise caused by resonance and vibration. There are three conventional methods for structural vibration control which can be classified as passive, active and hybrid.

\subsubsection{Passive Structure Vibration and Noise Control}

The passive control methods usually include an add-on method which put additional isolator such as absorbers, barriers, mufflers, dampers to separate the structure from external vibrating sources, then to reduce the vibration or noise in an enclosure (Ji et al., 2014). The second passive vibration control is design the system initially to avoid potential resonance (Torquato et al., 2003; Sreekala et al., 2010). Shape memory materials (SMA) such as nickel titanium (NiTi) alloys were used as passive vibration control for vibration control applications (Sreekala et al., 2010). For the existing structure with no possibility of isolation, damping is introduced by the addition of VEM onto the surface of the host structures (Xia, 1993; Masuda et al., 1997; Torvik, 2009). Viscoelastic materials have been applied to damping technologies from the second half of the twentieth century (Ferry, 1970; Ungar and Kerwin, 1962; Grootenhuis, 1970). 
Rao (2004) has described the applications of viscoelastic damping materials in automotive and aircraft structures. He stated that in a viscously damped system, the definition of the loss factor (or loss coefficient) $\eta$ is as:

$$
\begin{aligned}
& \eta=\frac{\left(\frac{\Delta W}{2 \pi}\right)}{W} \\
& =\left(\frac{\text { Energy dissipated during } 1 \text { cycle of harmonic displacement/radian }}{\text { Maximum strain energy in cycle }}\right.
\end{aligned}
$$

The amplitude of the response of the system at resonance $\left(\omega=\omega_{n}\right)$ is given by

$$
\frac{F_{0}}{k \eta}=\frac{F_{0}}{a E \eta}
$$

where $F_{0}$ is the amplitude, $E$ is the Young's modulus, and $a$ is a constant; The viscoelastic materials have larger values of the loss factor and hence are used to provide internal damping. When viscoelastic materials are used for vibration control, they are subjected to shear or direct strains. Equation (2.2) shows that a material with the highest value of $\eta$ gives the smallest resonance amplitude. Since the strain is proportional to the displacement $\mathrm{x}$ and the stress is proportional to $\mathrm{E}$ times $\mathrm{x}$, the material with the largest value of the loss factor will be subjected to the smallest stresses. The values of loss coefficient for some materials are given below: 
Table 2.1 Loss coefficient of materials (Rao, 2004).

\begin{tabular}{|l|l|}
\hline Material & Loss Factor $(\eta)$ \\
\hline Polystyrene & 2.0 \\
\hline Hard rubber & 1.0 \\
\hline Fiber mats with matrix & 0.1 \\
\hline Cork & $0.13-0.17$ \\
\hline Aluminum & $1 \times 10^{-4}$ \\
\hline Iron and steel & $2-6 \times 10^{-4}$ \\
\hline
\end{tabular}

With regards to adding VEM to the systems to have the passive vibration control effects, sandwich structures have always been constructed. There are two types of passive damping treatments for sandwich structures, namely free layer damping (FLD) and passive constrained layer damping (PCLD). FLD treatment means just one layer of VEM to one side or to both sides of the base structures, while PCLD treatments usually need to put an extra metallic layer upon the VEM layer as a constraining layer.

Free layer damping is the damping configuration which was treated by spraying or bonding the VEM layer to the host structure surface, either on one side or on both sides (See Figure 2.1 (a)). The material is simply attached with a strong bonding agent to the surface of a structure. Energy is dissipated because of extension and compression of the damping material under flexural stress from the base structure. Passive constrained layer damping (CLD) systems are usually used for very stiff structures (See Figure 2.1 
(b)). A "sandwich" is formed by laminating the base layer to the damping layer and adding a third constraining layer. When the system flexes during vibration, shear strains develop in the damping layer. Energy is lost through shear deformation, rather than extension, of the material.

The damping ratios obtainable with different types of construction/arrangement are indicated in Table 2.1.
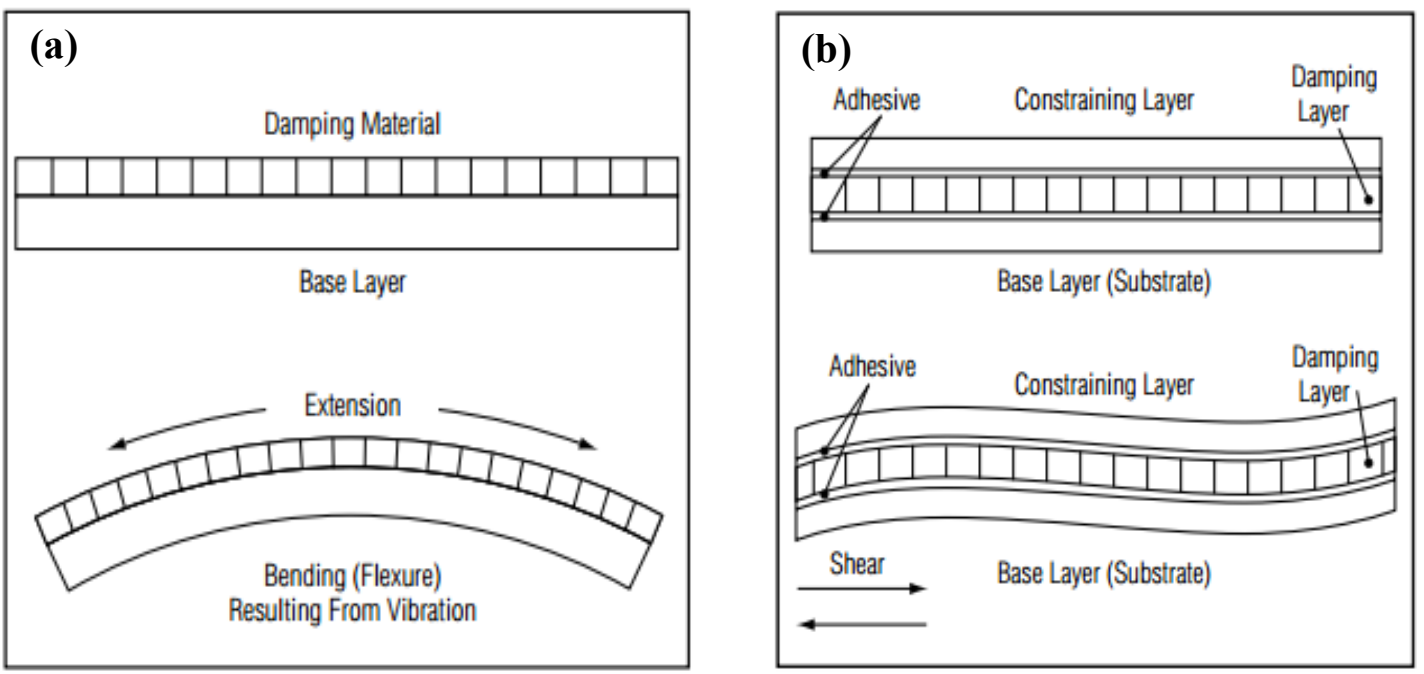

Figure 2.1 Damping treatment. (a) FLD treatments and (b) PCLD treatments (Renninger, 2004).

A disadvantage with the use of viscoelastic materials is that they are heavy along with the fact that their properties change with temperature, frequency, and strain. There are other materials that have been used for vibration damping properties. For example, MWCNT have been demonstrated to have damping properties (Li et al., 2015; Alva and Raja, 2014; Alnefaie et al., 2013; Tehrani et al., 2013; Hazarika and Maji, 2014; Xiong et al., 2006) no matter in free layer damping construction (Li and Levy, 2007b) and passive constrained layer damping construction (Koratkar et al., 2002). 
Table 2.2 Damping ratios of different types of construction/arrangement (Rao, 2004).

\begin{tabular}{|l|l|}
\hline Type of Construction/Arrangement & $\begin{array}{l}\text { Equivalent Viscous } \\
\text { Damping Ratio (\%) }\end{array}$ \\
\hline Welded construction & $1-4$ \\
\hline Bolted construction & $3-10$ \\
\hline Steel frame & $5-6$ \\
\hline Unconstrained viscoelastic layer on steel-concrete girder & $4-5$ \\
\hline Constrained viscoelastic layer on steel-concrete girder & $5-8$ \\
\hline
\end{tabular}

\subsubsection{Active and Hybrid Structure Vibration Control}

Different from passive vibration control, the active vibration control construction is to use a set of actuators, sensors and controllers connected by a feedback loop. Active vibration control methods include pure active control treatment and hybrid treatment. For the former treatments, it usually applies actuators in form of patches and metallic dampers directly mounted (or embedded) onto the vibrating structures, while for the latter one, they applied both sensor/actuators and passive VEM damping treatment at the same time.

There have been many investigations on the topic of pure active vibration control (Hong et al., 1992; Levy and Chen, 1994; Paulitsch et al., 2006; Boz and Basdogan, 2015; Takahashi et al., 2016). The advantages of this active vibration control are that the actuators, sensors and controllers can adapt the system to the environmental changes, 
such as temperature, frequency and strain, which restrained the passive vibration control. For example, most sensors found within active isolation systems are capable of detecting parameters such as velocity, acceleration and displacement. However, there are also some disadvantages for pure active vibration control. For example, the sensors, actuators or controllers depend on the external power to work, which is vulnerable to power failure. Another disadvantage is the additional sensors, actuators or controllers produced secondary control action upon the structure that will affect the effects of a primary excitation (disturbance).

Hybrid active/passive vibration control treatments are characterized by a blend of smart materials properties such as piezoelectric and viscoelastic damping capabilities. Reviews, surveys and assessments on hybrid active/passive vibration control treatments and related technologies can be found, for example, Park and Baz (1999), Trindade and Maio (2008) and Stanway et al. (2003). Therefore, hybrid active/passive vibration control treatments possess both passive vibration control treatments and pure active vibration control treatments, which is widely studied by researchers (Stanway et al., 1996; Stanway et al., 2004; Odden, 2010; Chen et al., 2001; Ikhouane and Dyke, 2007). There are also some other vibration control constructions been studied. Piezoelectric materials (PEM) have been studied as one of the actuators for pure active vibration control treatment. For example, piezoelectric materials have been studied to have vibration damping properties. Trindade research group (2008) use PCLD treatments found that shear piezoelectric materials added between two layers of steel (see Figure 2.2) could achieve good damping results by connecting to properly tuned 
resistive shunt circuits. A loss factor of $31 \%$ and average damping factors up to $1.5 \%$ are achievable, which could yield a reduction of approximately $20 \mathrm{~dB}$ in the vibration amplitude at resonance.
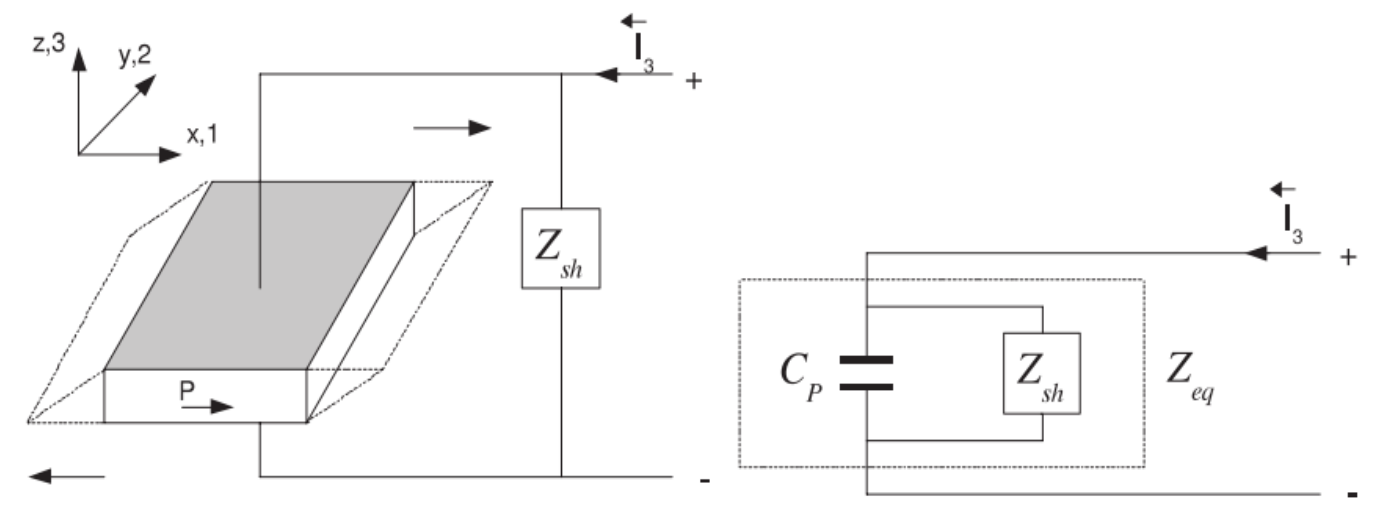

Figure 2.2 Representation of a piezoelectric capacitance in parallel with shunt impedance. piezoelectric patch represented as an equivalent capacitance (Trindade and Maio, 2008).

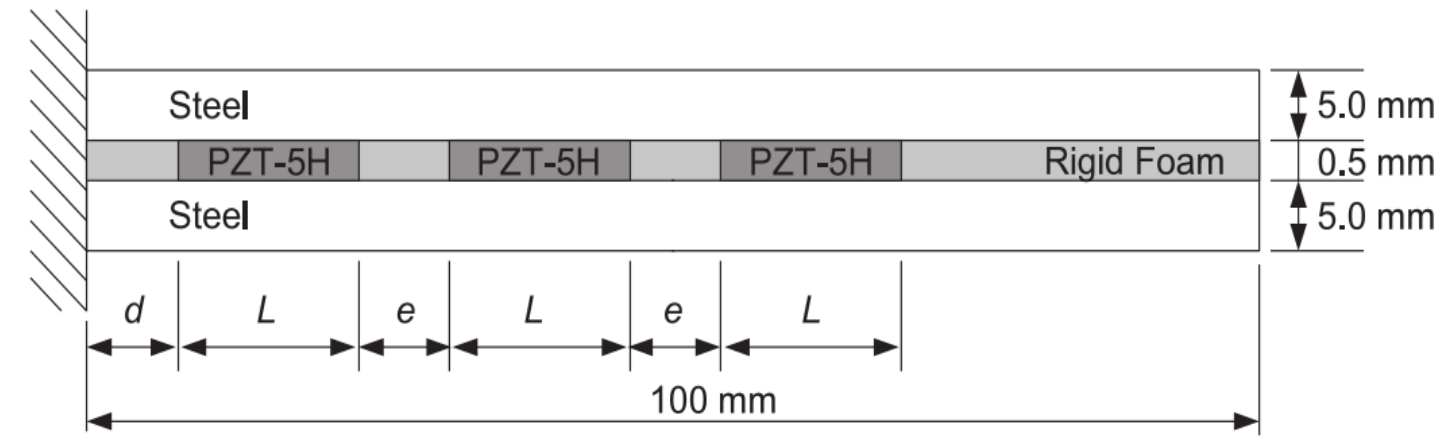

Figure 2.3 Schematic representation of the cantilever sandwich beam with three piezoelectric patches (not to scale) (Trindade and Maio, 2008).

\subsubsection{Active Vibration Control Using Strain Sensors}

As previously stated, the active vibration control construction is to use a set of actuators, sensors and controllers. In 1980, Crosby constructed a system which been monitored the vibration of a bowl centrifuge with a rotating conveyer consists of 
strain gages that are fixed on a bar which is connected, indirectly, to the gear box. In 1998, Varadan created a programmable wireless accelerometer by integrating MEMS, SAW devices and required microelectronics and conformal antenna, which can be used as strain sensors. In 2003, Michael Kehlenbach designed a piezo-ceramic actuator as piezo fiber modules and the fiber Bragg grating sensors, which are at least partially embedded in the piezo fiber modules. This invented actuator and sensor system is for composite structures, especially carbon fiber reinforced plastic structures with piezoceramic actuators, particularly for active vibration dampening and/or shape control purposes, as well as fiber Bragg grating sensors. In 2006, Kawabe research group constructed an active vibration control system, which consists of a three-layer-type neural network controller, a strain gauge sensor and an actuator, generate electromagnetically interactive control force. It is found that such active vibration control system has about 6.2 times the maximum damping effect observed in the Fe-based ferromagnetic high damping metal.

Ma and his coworkers (2011) found that polyvinylidene fluoride (PVDF) films have good performance as strain sensors by comparing them with conventional strain gauge through dynamic sensing test (see Figure 2.4 and 2.5). Also, they built four simple, robust active vibration controllers, which are all employed with the collocated PZT/PVDF pair, successfully suppressed the vibration of the cantilever beam subjected to impact loadings. And among those active vibration controllers, the integral resonant controller (irc) can sufficiently suppress all modes vibrations. 


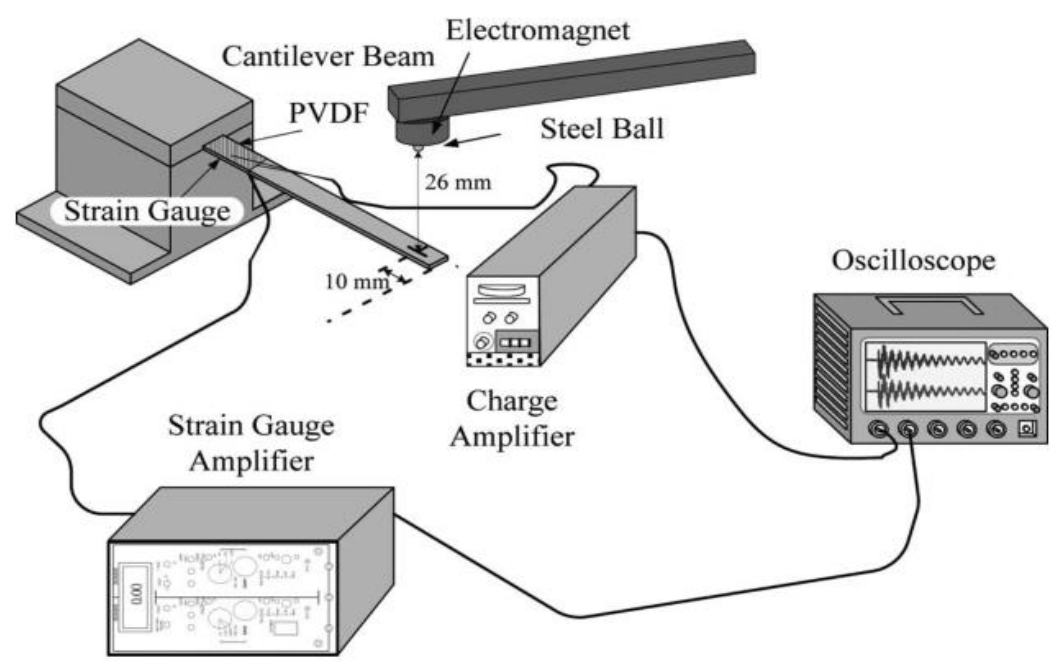

Figure 2.4 The experimental setup of the measurement system (Ma et al., 2011).

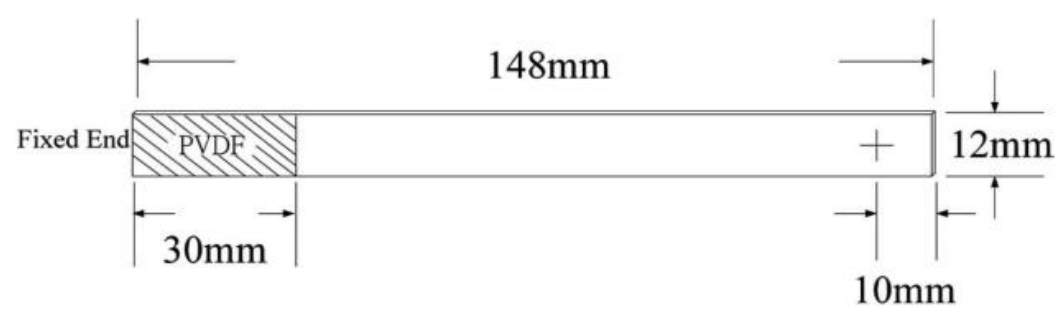

(a)

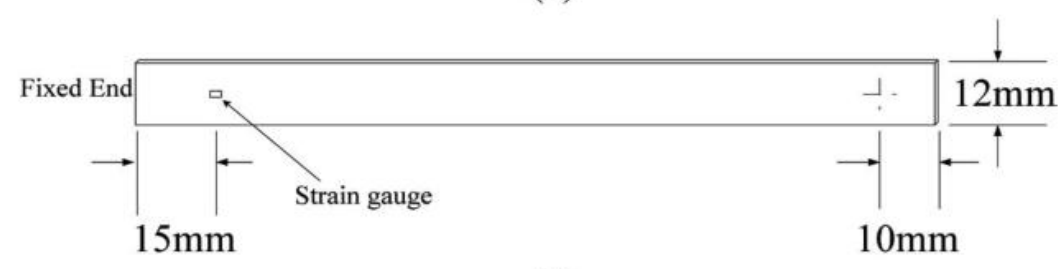

(b)

Figure 2.5 (a) The location of the PVDF sensor and the impact location on the top surface of the cantilever beam. (b) The location of the strain gauge on the bottom surface of the cantilever beam (Ma et al., 2011).

\subsection{Strain Sensors Research Background}

There are four main sensors that have been studied and used. They are electrostatic sensors, piezoresistive sensors, piezoelectric sensors and optical sensors. Among these sensors, piezoresistvie sensors refer to the change in resistance upon an induced strain and deformation. By strict definition, piezoresistors refer to devices in which change in 
resistance is caused by alteration in resistivity (Chang Liu, 2005). A device in which resistance change is mainly by the dimension deformation is referred to as a strain gauge. However, researchers showed great interests in the strain sensing ability of polymer composites which depends markedly on external loading (see Lundberg and Sundqvist (1986), Carmona et al. (1987), Pramanik et al. (1990), Yoshikawa et al. (1990), McLachlan et al. (1990), Taya et al. (1994) and Carmona and Amarti (2016)).

Carbon nanotubes have many excellent properties, especially their extraordinary mechanical properties and electrical conductivities have been studied widely recently (Hu et al., 2007; Li et al., 2009, 2010). Therefore, CNT are ideal structural component candidates in various composites and functional composites because of their mechanical reinforcement effects (Hu et al., 2005, 2012; Li et al., 2011; Liu et al., 2011). Also, it will produce conductive polymer/CNT nanocomposites with CNT dispersed in the insulating polymers. Such electrically conductive CNT/polymer nanocomposite can be applied to various fields, for example, resistance-type strain sensors of high sensitivity or piezoresistive, electromagnetic interference materials. When the SWCNT are used as resistance-type strain sensors, it was found that their electrical conductivity could be dramatically changed by introduction of strain using atomic force microscopy (AFM), as a consequence of the band-gap and structural changes under the effect of mechanical strain (Tombler et al., 2000). After that, CNT were found to have strain sensing properties by many researchers (Dharap et al., 2004; Li et al., 2004; Kang et al., 2006; Rein et al., 2011). Furthermore, as mentioned before, Li and Levy's (2008) preliminary research results have indicated that buckypaper 
(MWCNT films) sandwiched with two electrical non-conductive adhesives were capable of being strain sensors.

Thus, CNT have been confirmed to have the piezoresistivity properties. Consequently, CNT aroused great interest of worldwide researchers in building new types of polymer/CNT composites strain sensors. In 1996, Shui and Chung first found that the relationship between strain and the fractional increase in electrical resistance $\left(\Delta \mathrm{R} / \mathrm{R}_{0}\right)$ of piezoresistive polyether-sulfone-matrix composite strain sensors was much more linear and less noisy when the electrically conducting filler was $0.1 \mu \mathrm{m}$ diameter carbon filaments rather than the conventionally used $10 \mu \mathrm{m}$ diameter carbon fibers. After that, the nanocomposition made by CNT combining/blending with different polymers has found to have the capability of strain sensors (Frogley et al., 2002; Ramaratnam and Jalili, 2006; Zhang et al., 2006; Park et al., 2008; Loh et al., 2008; Hu et al., 2008; Wichmann et al., 2008, 2009; Lin et al., 2015, 2016).

Ramaratnam and Jalili (2006) studied the strain sensing ability of the piezoelectric polymers Poly (vinylidene fluoride-trifluoroethylene) $\quad(\mathrm{P}(\mathrm{VDF}-\mathrm{TrFE})$ ) /CNT nanocomposites using free end vibration test method (see Figure 2.6). The experimental results along with theoretical verification showed that the nanotube-based polymers yielded better response characteristics than those of the plain piezoelectric polymers. Also, he stated that the sensing performance of the PVDF/CNT nanocomposites improved by the increased of the Yong's modulus of the system, which the comparisons been made between plain $\mathrm{P}(\mathrm{VDF}-\mathrm{TrFE})$, multiwalled carbon nanotube (MWCNT)/ $\mathrm{P}(\mathrm{VDF}-\mathrm{TrFE})$ and SWCNT. 


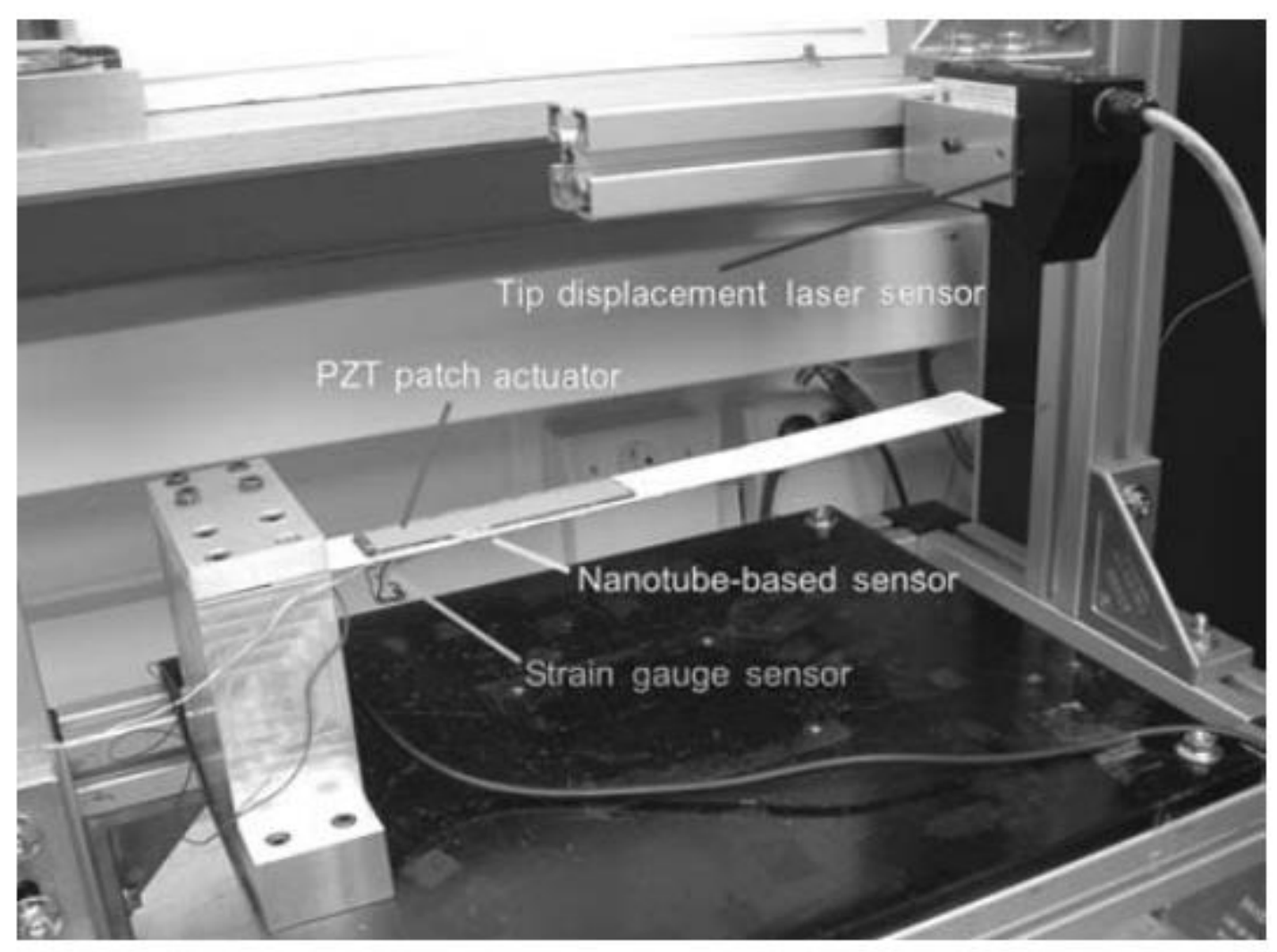

Figure 2.6 Experimental setup for testing the proposed nanotubebased sensors (Ramaratnam and Jalili, 2006).

2.4 Background of Multifunctional Materials Having Both Structural Damping Properties and Strain Sensing Capabilities

The studies on multifunctional materials having both structural damping properties and strain sensing properties are very important in many fields. Specifically, it is a key problem in structural health monitoring domains. Although, hybrid active/passive vibration control treatments created such multifunctional materials as stated in section 2.2.2 of this chapter, there are some limitations which are given below: 
(1) The conventional VEM are temperature and frequency dependent, which narrows the application of such systems; (2) the conventional VEM always has considerable weight, which sometimes restricts its used in certain situations, for example when applied to aeronautical and aerospace engineering, where a little weight will dramatically increase the entire cost; and (3) for hybrid vibration control treatments, which is so called "add-on" methods, there will be additional isolators added such as absorbers, barriers, dampers to separate the structure from external vibrating sources, thus reducing the vibration in an enclosure. Therefore, technologies and methods to make new multifunctional materials with both structural damping properties and strain sensing properties are needed.

Carbon nanotube systems have been found to have the capability of being multifunctional materials for both structural damping properties and strain sensing properties (Li and Levy, 2007b; Chen and Weng, 2007). However, there are few researchers who have directly studied the multifunctional properties of polymer composites which have both structural damping properties and strain sensing properties (Ramaratnam et al., 2004; Kawabe et al., 2006; Tian and Wang, 2008; Tian et al., 2008; Hazarika and Maji, 2014). However, there is huge potential for them possessing both properties, especially for the CNT, carbon nanoparticles, carbon nanofiber or even graphene modified polymers nanocomposites. For examples, epoxy composites modified with carbon nanoparticles (Wichmann et al., 2009), epoxy composites modified with carbon nanotube (Wichmann et al., 2008; Yin et al., 2011), epoxy composites modified with carbon nanofiber (Shimamura et al., 2011), and even epoxy 
based on grapheme (Kim et al., 2011), all have been confirmed as being capable of strain sensing. And those epoxy based composites have already been studied for their good structural damping properties (Suhr et al., 2005; Alnefaie et al., 2013; Alva and Raja, 2014). Similarly, carbon nanotube/polycarbonate composites (Zhang et al., 2006), polyurethane/carbon nanotube composites (Chang et al., 2010), polypropylene/carbon nanofibre composites (Costa et al., 2014), polyamide-6/ carbon nanotube composites (Meincke et al., 2004), and others have been studied for their strain sensing properties (Shui and Chung, 1996). And those polymer composites have also been studied for their structural damping properties by other researchers (Liu et al., 2010; Li et al., 2016; Finegan et al., 2003; Kireitseu, 2006).

Although, there are many advantages for polymer/CNT nanocomposites to be multifunctional materials having both structural damping properties and strain sensing properties, there are still several problems encountered. Examples encountered include the difficulty dispersing CNT into the polymer matrix and the electrical non-conductive properties of most polymers. Therefore, methods and technologies to make good dispersion of polymer/CNT needed to be found.

\subsection{Polyaniline/MWCNT and Polyurethane/MWCNT}

\subsubsection{Polyaniline/MWCNT}

Polyaniline was discovered over 150 years ago, however, only since the early 1980s has polyaniline captured the intense attention of the scientific community mainly due to the rediscovery of its high electrical conductivity (Okamoto and Brenner, 1964). 
Also, PANI is the cheapest polymer among its conducting polymer family, which has a rich chemistry.

Most polyaniline/carbon nanotube composites have been invented and characterized with their electrochemistry properties and have been confirmed as capable of supercapacitor applications (Simotwo et al., 2016; He et al., 2016; Tran et al., 2016). And some other researchers studied their properties as catalysts, such as photocatalytic properties (Wang et al., 2016) and electrocatalytic properties (Liu et al., 2016; Zhao et al., 2015; Li et al., 2015). Narayanankutty (2015) research group found that the well-dispersed filler in functionalized MWCNT (FMWCNT)-PANI/ thermoplastic PU (TPU) (I) composites improved strain sensitivity. They stated that the coating of PANI on FMWCNT reduced its entanglement and enhanced the interfacial interaction between the nano fillers and TPU, leading to improved strain sensitivity. And some other investigators (Huang et al., 2014) also showed that PANI composites has superior strain sensitive properties. Furthermore, polyaniline composites were found to have the damping properties (He et al., 2008; Kim et al., 2001).

There are a few investigations in which both of their damping and strain sensing properties are being studied. This is because, up to now, most polyaniline/carbon nanotube (PANI/CNT) nanocomposites materials obtained by the chemical methods were in powder form (Huang et al., 2010; Qiao et al., 2007). Though some papers stated that binding materials were used when making PANI/CNT nanocomposites (Huang et al., 2010; Wang et al., 2007; Lu et al., 2011), the binding materials were thought to deteriorate the chemical or mechanical properties of PANI/CNT nanocomposites films. 
Furthermore, because it is difficult to control the thickness of PANI/MWCNT nanocomposites films, there are very few papers reporting their damping properties.

It suggested that in situ polymerization is an ideal technique to make a perfect dispersion of carbon nanotube into polymer matrixes. In situ polymerization of aniline with the presence of MWCNT solved the MWCNT dispersion problems (Tan and Xu, 2016). Frit compression is a technique used to fabricate buckypaper and buckydiscs from a suspension of carbon nanotubes in a solvent. The Whitby research group (2008) first used the frit compression method to successfully fabricate MWCNT buckypaper/buckydiscs. Buckypaper has been created using MWCNT in a water suspension with surfactant added technique. Compared with surfactant casting or acid oxidation filtration methods, the buckypaper/buckydiscs created by the frit compression method avoids any surfactant or surface modification techniques. Also, the distribution of CNT in solvent did not need to be a thermally stable suspension. A general dispersion of CNT in solvent will keep the nanotubes between the frits rather than allowing them to pass through the frits. This methodology rapidly speeds up the casting process and the solvent can be fully recovered, making it an efficient method to fabricate polymer composites films/discs.

\subsubsection{Polyurethane/MWCNT}

In 1937, Otto Bayer and his coworkers at IG Farben in Leverkusen, Germany, first made polyurethanes (Bayer, 1937; Seymour and Kauffman, 1992). In the early work, PU was used as a coating during World War II. Polyisocyanates became commercially 
available in 1952, and production of flexible polyurethane foam began in 1954 using toluene diisocyanate (TDI) and polyester polyols. PU is well-known as 'wearresistant rubber' and has excellent flexibility, anti-friction, high strength, oil resistance, etc. The dynamic properties of PU along with its high load-bearing capacity make it an excellent choice for many vibration control applications.

In 1992, Kogelnik research group compared the polyurethane elastomers with rubber when they are both used in automotive and nonautomotive applications as damping components. They found polyurethane elastomers has many advantages over rubber both in static and dynamic applications. For examples, it shows high-volume compressibility, lighter weight, better flexibility, smaller size with similar performance, and resistance to ozone UV light and petroleum products. Recently, Stegmaier, Peter (2014) invented a supporting and vibration damping component which was comprised of polyurethane elastomer by a novel method. Toker, Mehmet (2014) presented an invention that relates to a seismic isolator that exhibits resistance against the dynamic and static vertical column loads and against the horizontal seismic shear forces experienced by the structures during an earthquake, absorbs these forces, does not transmit forces to the building, is reinforced with steel metal based plates and is laminated by the solid polyurethane elastomer materials. Jing (2015) demonstrated a synthesizing method which made polyurethane elastomer vibration damper plates used in high-speed railway, which have good vibration damping function, good buffer property, stretch resistance, tear resistance, mechanical property and high hardness and can adapt to wide temperature range. 
Recently, many investigations have been focused on inventing the synthesizing method and applications of carbon nanotube modified polyurethane (Yan et al., 2016; Oh et al., 2016; Fang et al., 2015; Li et al., 2016). Sasikumar research group (2012) reported that the PU/ MWCNT nanocomposites have good damping properties by characterizing with the DMA and FLD methods. The group demonstrated that the effectiveness of the developed nanocomposite which has good damping of the order of 5-15 $\mathrm{dB}$ is observed in the frequency range of $10-1000 \mathrm{~Hz}$, over that of neat PU. And the added advantage of the system is the damping capability is also in the low frequency region. Such composites are expected to be helpful in finding damping applications in aircraft and missile substructures, ships and submarines, machinery supports, mounting platforms of electronic equipment, bridges, buildings, etc. Bandarian research group (2011) studied that only $0.1 \%$ MWCNT dispersed in PU will dramatically increase their damping properties.

However, the dispersion of MWCNT in the polyurethane is a concern. So, some researchers such as the Yuqi Li research group (2015) used the in situ polymerization in the presence of polyisobutylene-based polyurethane MWCNT method to get a well dispersed MWCNT/polyisobutylene-based polyurethane, which also displayed good vibration damping properties. Zhan (2010) used a novel minimixer to make PU/MWCNT nanocomposites. The dynamic mechanical analysis results showed that the storage modulus at room temperature was increased by adding with $1 \%$ MWCNT in PU. 
2.6 Novelty of The Present Work

This dissertation work addressed two main issues, which were the damping properties and the strain sensing properties of PANI/MWCNT and PU/MWCNT buckypaper.

Firstly, in situ polymerization of PANI with the presence of the MWCNT was carried out to solve the dispersion issue of the MWCNT in the polymer matrix. For PU/MWCNT composites, layer-by-layer method was used to get good electrical conductive samples. The damping properties of both PANI/MWCNT and PU/MWCNT composites were tested by the free vibration method. Both composites were found to show good performance on the vibration damping systems. Several attachment methods of the PANI/MWCNT and PU/MWCNT samples onto the aluminum base beam were employed and the results were compared.

Also, the second main issue to be evaluated was the strain sensing property. Our work demonstrated that PANI could be used for strain sensing. As mentioned previously, a specific type of PANI is usually synthesized, which has led to the technical difficulty of setting up the experiment to do both the strain sensing tests and the free end vibration test. Frit compression method was used in this dissertation to make either circular or rectangular samples on which those tests were carried out. Also, to get the electrical conductivity of the PU/MWCNT, a simple layer-by-layer method was used to successfully set up the static and dynamic strain sensing test. Our results have been found to follow the trends discussed by Rao ( 2004) and Levy and Chen (Levy and Chen, 1994; Chen and Levy, 1998) for constrained viscoelastic material layers. 
CHAPTER III

PREPARATION OF CIRCULAR POLYANILINE/MULTIWALLED CARBON NANOTUBE AND CHARACTERIZATION OF THEIR STRUCTURAL DAMPING

\section{AND STRAIN SENSING PROPERTIES}

\subsection{Introduction}

Our initial attempt to evaluate PANI/MWCNT for strain sensing and damping had to overcome the fabrication issues discussed in the previous chapters. It was for this reason that the process began by fabricating circular samples using a simple fabrication method. In situ synthesizing method was used to fabricate PANI/MWCNT. Frit compression method was used to make circular PANI/MWCNT samples. The reason we made circular samples here is because they were easy to fabricate (Whitby et al., 2008). The thickness of these PANI/MWCNT films/discs can also be controlled. The properties of electrical resistance and thermal stability of these films/discs are characterized and the mechanical properties, such as Young's modulus and hardness of the nanocomposite films/discs, are discussed. Furthermore, their preliminary damping and strain sensing properties are evaluated.

\subsection{Experimental Sections}

\subsubsection{Surface Treatment of MWCNT}

Two kinds of surface treatment of MWCNT were carried out: dry heat treatment and mixture acid oxidation to purify MWCNT and form ketone and acid groups. The experimental details are described below: 
Dry heat treatment of MWCNT. Eight grams of MWCNT was spread equally into the bottom of a $2 \times 4 \mathrm{~cm}$ ceramic bowl. The bowl was put into a muffle furnace for 80 minutes at a temperature of $450{ }^{\circ} \mathrm{C}$ for refinement. Figure 3.1 shows the heat graph of the muffle furnace.

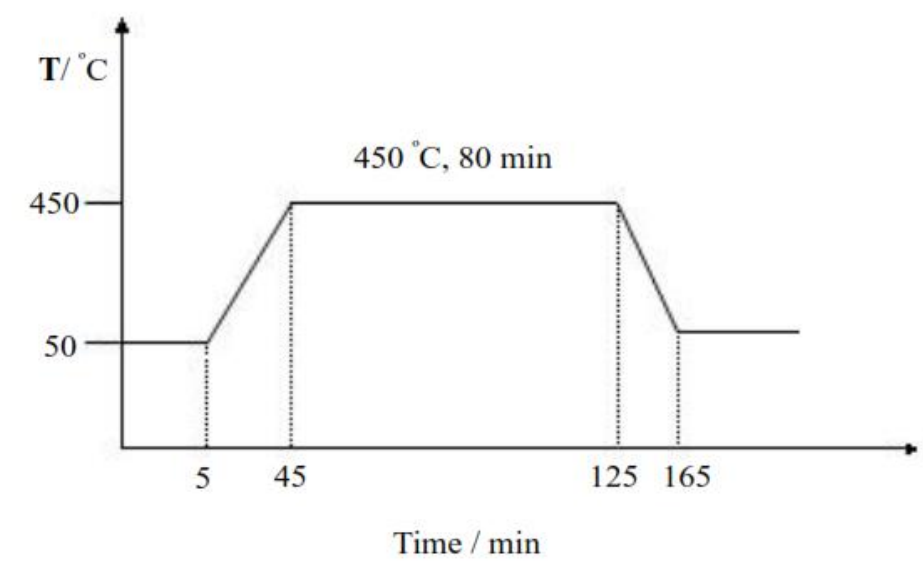

Figure 3.1 The dry heat treatment processing of MWCNT

Chemical modification of MWCNT. After heat treatment, a mixed acid solution $\left(\mathrm{H}_{2} \mathrm{SO}_{4}: \mathrm{HNO}_{3}=3: 1\right)$ treatment under supersonic bath was used to improve the MWCNT purity by removing metal catalyst impurities (Tasis et al., 2006), and to improve interfacial adhesion with polymer matrix by surface oxidation of the MWCNT to form ketone and acid groups. A large amount of distilled water was added to the MWCNT suspension. After cooling down, the suspension was added into centrifuge tubes and centrifuged at $4000 \mathrm{rpm}$ for $10 \mathrm{~min}$. The black MWCNT was precipitated to the bottom of the centrifuge tube. The top acid solution was safely discarded and the precipitate of MWCNT remained. The MWCNT precipitate was washed with distilled water at least five times to reach a $\mathrm{PH} \approx 7$. Its neutralization was checked with $\mathrm{pH}$ measurement paper. The washed MWCNT were dried in vacuum at $60{ }^{\circ} \mathrm{C}$ for more than $12 \mathrm{hrs}$. 


\subsubsection{In situ synthesizing of circular PANI/11\% MWCNT composites}

$64.375 \pm 0.010 \mathrm{mg}$ of MWCNT (Newton, MA, www.nano-lab.com : PD15L5-20) with length of 5-20 $\mu \mathrm{m}$ and outer diameter of $15 \pm 5 \mathrm{~nm}$ were dispersed by sonication in $125 \mathrm{ml}$ of $1 \mathrm{M} \mathrm{HCl}$ for $30 \mathrm{~min}$. Then, $1.287 \pm 0.003 \mathrm{~g}$ of aniline was added and the mixture was sonicated for another $30 \mathrm{~min}$. After that, $1.027 \pm 0.003 \mathrm{~g}$ of ammonium persulphate (APS) dissolved in $125 \mathrm{ml}$ of $1 \mathrm{M} \mathrm{HCl}$ was added at once and polymerization was conducted at $0{ }^{\circ} \mathrm{C}$ with water-ice bath under sonication for $4 \mathrm{hrs}$. The precipitated products were washed with deionized water. Then the PANI/ $11 \% \mathrm{wt}$ MWCNT composite was made. Here we use the column frit compression to compress the sample to remove the excess liquid. The details are as follows:

The PANI/MWCNT nanocomposites films were made by using frit compression methods. Certain amount of obtained PANI/MWCNT suspension was transferred into $50 \mathrm{ml}$ column, which was equipped with 70 micrometer pore diameter frit. When squeezing out the solvent, the frits- PANI/MWCNT sandwich with the syringe column was dried in a $110{ }^{\circ} \mathrm{C}$ oven for $12 \mathrm{~h}$. The disc-like PANI/MWCNT nanocomposites film was obtained when removed from the syringe housing. The disc-like MWCNT film was made by means of the same procedures. Four different components of nanocomposites were synthesized by the same procedures (See Table 3.1).

In addition, the pure raw MWCNT film was made by the same frit compression procedure. About $0.4 \mathrm{~g}$ pure MWCNT was put into a certain amount of deionized water. Then the suspension was transferred into the syringe column to squeeze out the solvent. 
The MWCNT sample was dried in the same manner as the PANI/MWCNT, and the disc-like MWCNT film was obtained by removing it from the syringe mold.

Table 3.1 Different components of nanocomposites films

\begin{tabular}{|l|l|l|l|}
\hline & MWCNT / mg & Aniline /g & APS / g \\
\hline Pure PANI & 0 & 1.28 & 1.03 \\
\hline PANI/11\% MWCNT & 50 & 1.14 & 0.91 \\
\hline PANI/22\% MWCNT & 100 & 1.00 & 0.80 \\
\hline PANI/44\% MWCNT & 200 & 0.72 & 0.57 \\
\hline Pure MWCNT & 454.54 & 0 & 0 \\
\hline
\end{tabular}

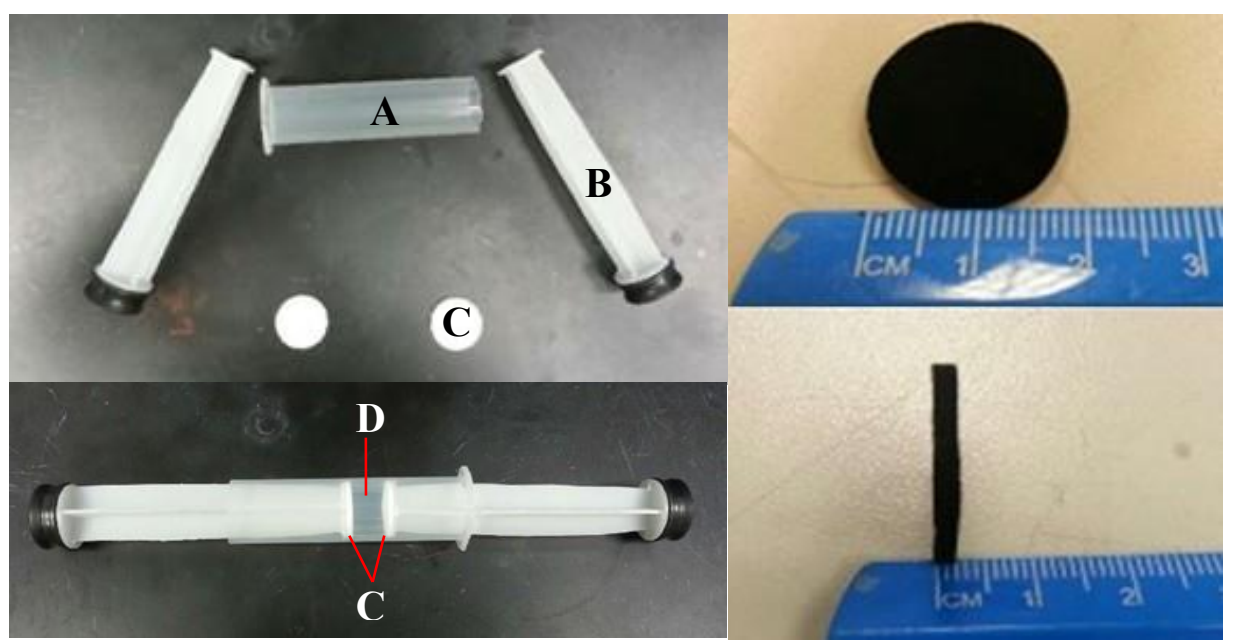

Figure 3.2 (left) A frit compression system for making nanocomposites films. (a) syringe column; (b) plungers; (c) polypropylene frits and (d) composites suspension. (right) Photo images of PANI/MWNT nanocomposites dics.

The polymer compositions we used here was PANI/11\% MWCNT with the radius of $1.9 \mathrm{~cm}$ and thickness of $1.7 \mathrm{~mm}$, which were bonded to the aluminum beams by 
nonconductive 3M CA100 liquid instant adhesive. There were three sizes of aluminum beams used as the base beam. The width and thickness of the beams were the same, namely, $2.5 \mathrm{~cm} \times 0.0051 \mathrm{~cm}$. The shortest distance from circular sample's edge to the end of the aluminum beam was fixed by $0.8 \mathrm{~cm}$. However, the length of the beams was $6 \mathrm{~cm}$ (see Figure 3.2 (left)), $9.3 \mathrm{~cm}$ and $12.6 \mathrm{~cm}$. Three very, thin layers of adhesive were used to cover these samples to separate samples from the environment. Each specimen was dried at room temperature for $24 \mathrm{~h}$.

\subsubsection{Characterization}

Transmission electron microscopy (TEM) and scanning electron microscopy (SEM) were used to their morphology properties. Two probe electrical measurement was used to study the electrical conductivity of different composition of PANI/MWCNT composites. Thermal stability of the PANI/MWCNT was studies by simultaneous DSCTGA (SDT) test. Free end vibration test method was used to study the damping properties of these samples. The location of the samples and the size of aluminum (Al) base beams were studied to test the damping properties of the PANI/MWCNT nanocomposites. Wheatstone bridge methods was used to test strain sensing capability of PANI/ MWCNT composites. The traditional foil strain gauge was used to compare with PANI/ MWCNT composites disc that were made.

\subsubsection{Damping Test Setup}

Free vibration displacement measurement was adopted for the damping test. The damping ratio was determined from data taken from vibration tests. The PSV-400 
PolyTech Laser Vibrometer was used to obtain vibration wave velocity data. Five different ratios of prepared PANI/MWCNT films were using $3 \mathrm{M}$ nonconductive adhesive glued on two different types of aluminum beams. Figure 3.3 shown the digital pictures of attached samples in different aluminum base beams.

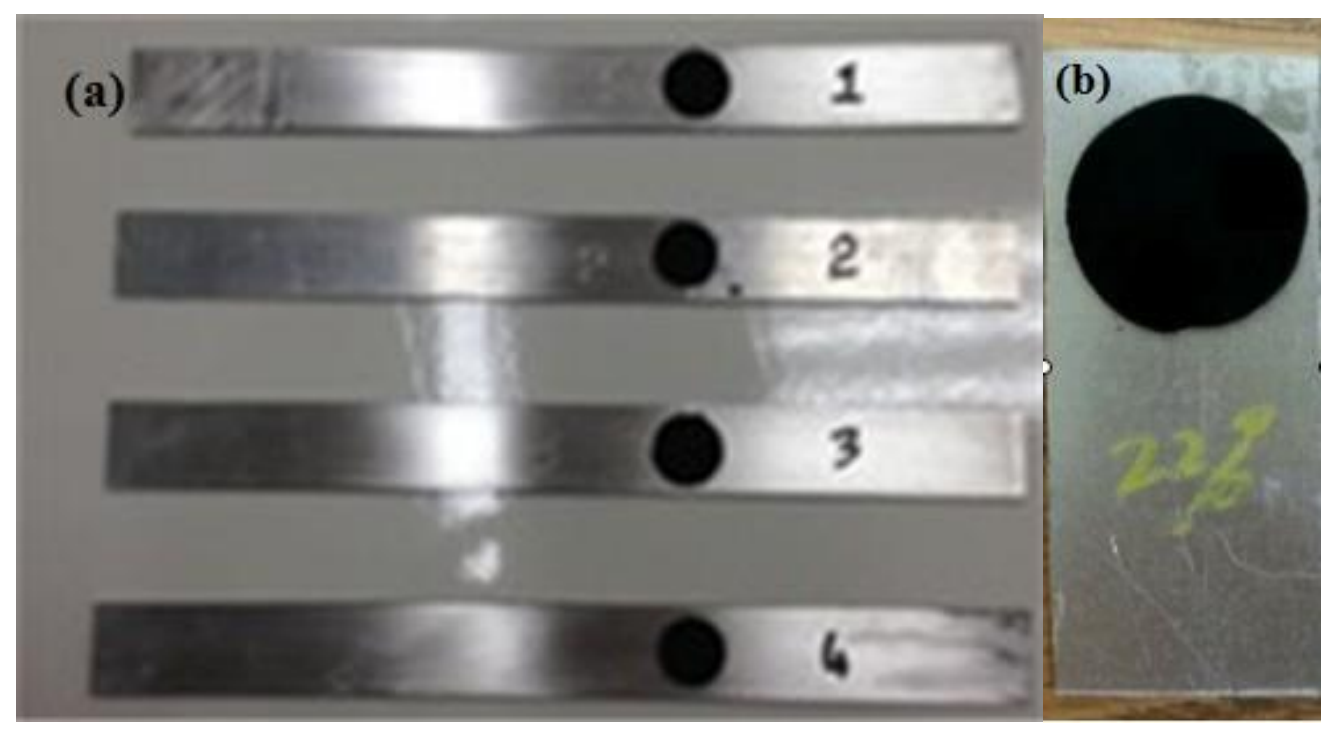

Figure 3.3 Digital pictures of attached samples on different aluminum beams with dimension by (a) 5.13 inch $\times 1$ inch $\times 0.12$ inch, and (b) 2.4 inch $\times 1$ inch $\times 0.008$ inch.

PANI/11\% MWCNT with the radius of $1.9 \mathrm{~cm}$ and thickness of $1.7 \mathrm{~mm}$ were used to study their location's effect on damping ratio when attached upon 2.4 inch $\times 1$ inch $\times 0.008$ inch Al base beam. The symbols \# and * indicate the location of the sample and are called sample side and free end side, respectively. Red lines were the locations on the sample side \# and free end side * where the beam is held fixed, and is shown in Figure 3.4.

The red line shown in Figures 3.4 (b), 3.4 (c) and 3.4 (d) indicated the places where the beam was fixed. Cantilever beams were given the same, small initial displacement 
and then released for free vibration. Free end displacements were measured by a laser vibrometer, and a two-channel digital oscilloscope was used to display and store the signal.

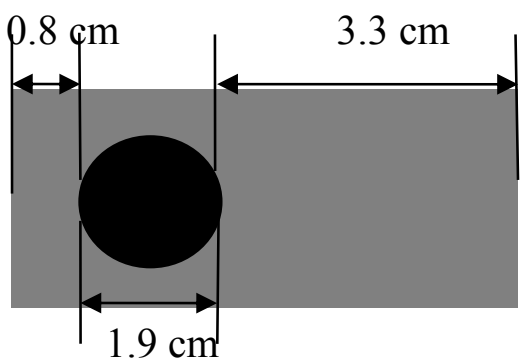

(a)

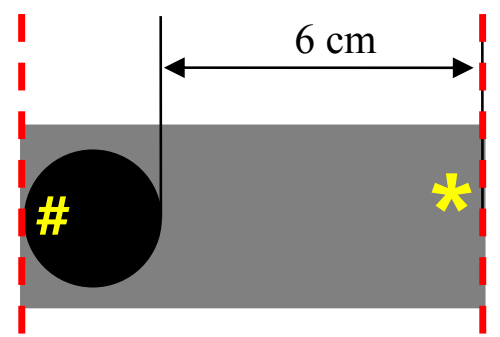

(c)

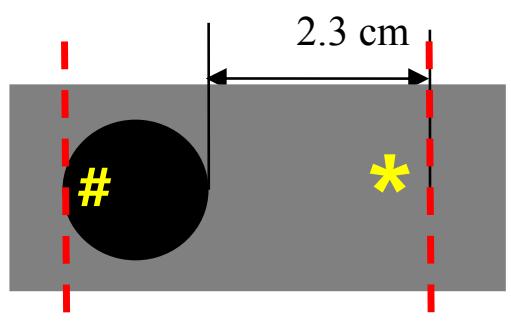

(b)

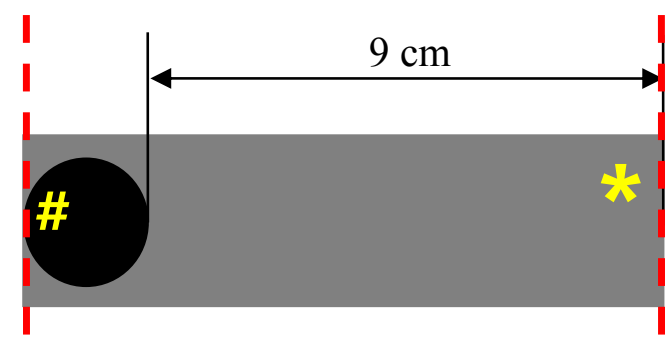

(d)

Figure 3.4 (a) Dimension of samples, aluminum beams, the locations where samples were held fixed. (a) and (b) $6 \mathrm{~cm}$ long beam (c) $9.3 \mathrm{~cm}$ long beam with fixed ends 0.8 $\mathrm{cm}$ from the left-hand side OR $0.6 \mathrm{~cm}$ from the right-hand side; and (c) $12.6 \mathrm{~cm}$ long aluminum beams with fixed ends $0.8 \mathrm{~cm}$ from the left-hand side or $0.9 \mathrm{~cm}$ from the right-hand side. Red lines were the locations sample side \# and free end side * where the beam is held fixed.

\subsubsection{The Strain Sensing Experiment Setup}

The samples PANI/MWCNT with the radius of $1.9 \mathrm{~cm}$ and thickness of $1.7 \mathrm{~mm}$ were attached onto the $26.67 \mathrm{~cm} \times 2.54 \mathrm{~cm} \times 0.3175 \mathrm{~cm}$ aluminum beam. Samples covered with a three-thin layer of electrical non-conductive adhesive (glue) were studied. The aluminum beam was fixed on the lab table. 
The wires from the sample were connected to the Wheatstone bridge box. Loads of $2.4525 \mathrm{~N}, 4.4145 \mathrm{~N}, 6.3765 \mathrm{~N}, 8.3385 \mathrm{~N}$ and $10.3005 \mathrm{~N}$, equivalent to weight loads of $250 \mathrm{~g}, 450 \mathrm{~g}, 650 \mathrm{~g}, 850 \mathrm{~g}$ and $1050 \mathrm{~g}$, were applied to the end of the aluminum beam, which led to the bending of the beam and the sample attached onto it. The strain change of the samples was calculated for each load. Finally, the data were recorded for each load and V output read directly from the Wheatstone bridge.

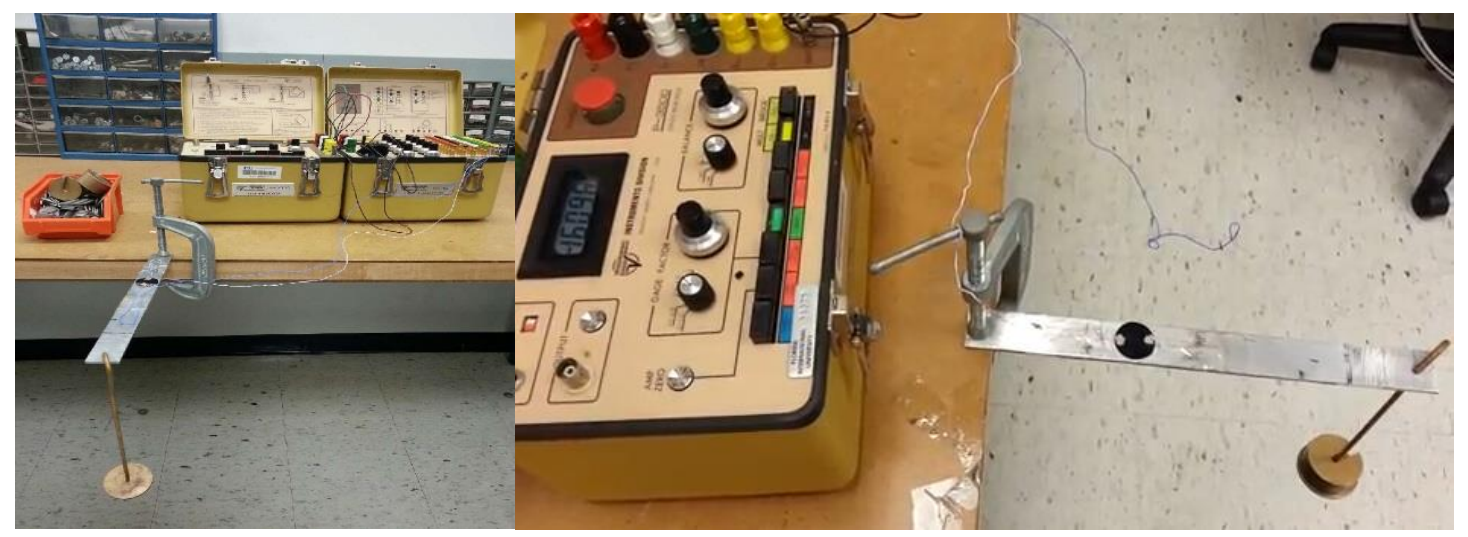

Figure 3.5 Strain sensor test setup.

\subsection{Results and Discussion}

\subsubsection{Electrical Conductivity of the PANI/MWCNT Nanocomposites Film}

The electrical resistance of the composites was measured because these were needed to correlate with the strain sensing capability of the composites. The electrical resistance (R) of PANI/11\% MWCNT, PANI/22\% MWCNT, PANI/44\% MWCNT, pure PANI and pure MWCNT films were measured by a multimeter at room temperature. The measure distance between two electrodes for measurement is $1.5 \mathrm{~cm}$. Three samples were measured for each composites film and the mean values and deviations are shown in Figure 3.6. 


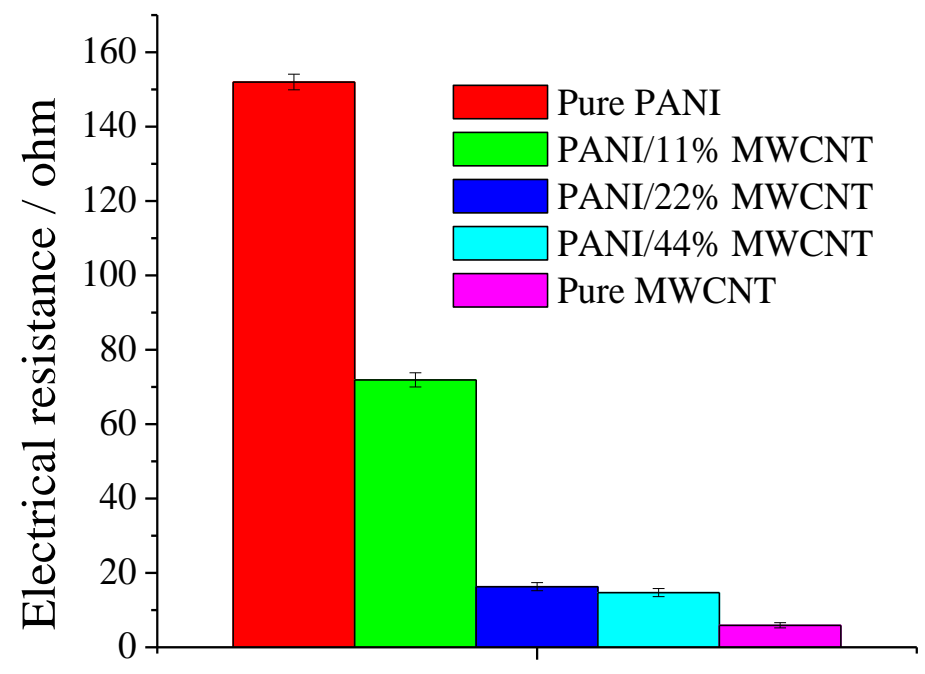

Figure 3.6 Electrical conductivity and standard deviations of PANI/11\% MWCNT, PANI $/ 22 \%$ MWCNT, PANI $/ 44 \%$ MWCNT, pure PANI and pure MWCNT films measured by multimeter.

It is found that the electrical resistance of pure MWCNT film was $5.9 \Omega$, while the pure PANI film's resistance is $152 \Omega$ showing the highest $R$ value compared to the other four films. The resistance of PANI/11\% MWCNT was reduced to around $70 \Omega$. Lower resistances were achieved by adding more MWCNT into the composition. The resistance values of PANI/22\% MWCNT and PANI/44\% MWCNT are about $16.3 \Omega$ and 14.6 $\Omega$, respectively, which are close. It indicated that the MWCNT network was firmly established when $22 \%$ MWCNT was added into the composition and adding more MWCNT would change the conductivity of the composition films marginally.

\subsubsection{Mechanical Properties of the PANI/MWNT Nanocomposite Films}

A nanoindentation test was used to study the mechanical properties of PANI/22\% MWCNT nanocomposite films. Six different locations were randomly selected to do the test. Figure 3.7 shows the most typical curve of the test. 


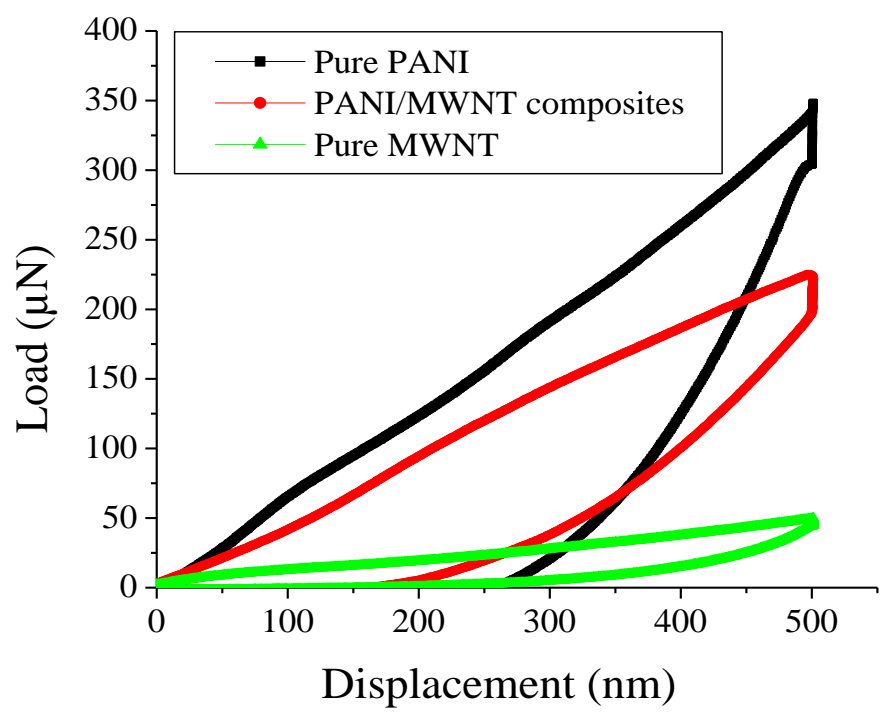

Figure 3.7 Nanoindentation (loading-unloading curves) of pristine PANI, pure MWCNT and PANI $/ 22 \%$ MWCNT nanocomposite film.

The hardness standard deviation of PANI/22\% MWCNT nanocomposite films was 0.0066 GPa and the Young's modulus deviation was $0.0803 \mathrm{GPa}$. This can be explained by the random spaghetti-like arrangement of PANI/MWCNT microstructure that resulted in local porosity and agglomerates. Also, the nanoindentation test results clearly showed that the PANI greatly contributed to increase the Young's modulus and hardness values of the PANI/22\% MWCNT nanocomposite films.

\subsubsection{Thermal Stability of the PANI/MWCNT Nanocomposites Films}

Thermal stability of the composite was evaluated because one of the primary tasks of the sample would be its use in high temperature applications. The results of SDT test in nitrogen are shown in Figure 3.8. It could be seen that the pure MWCNT could not be decomposed until the temperatures reached around $550{ }^{\circ} \mathrm{C}$, while the pure PANI started to decompose when the temperature reached $350{ }^{\circ} \mathrm{C}$. 
There are two major stages of weight loss for the polyaniline, regardless of the experimental environments. The first weight loss occurs at the lower temperature of about $50{ }^{\circ} \mathrm{C}$ and results from moisture evaporation and outgassing of unknown small molecules like water, carbon dioxide, etc. The second weight loss indicates a structural decomposition of the polymer and occurs at the higher temperature which starts at about $350{ }^{\circ} \mathrm{C}$. For PANI $/ 22 \%$ MWCNT composites, there are two strong exothermic peaks at $400{ }^{\circ} \mathrm{C}$ and $600{ }^{\circ} \mathrm{C}$, which correspond to the exothermic peak of PANI and MWCNT, respectively. It could be easily concluded that the thermal stability of the PANI/ $22 \%$ MWCNT was improved by about $50^{\circ} \mathrm{C}$. It also shows two major stages of weight loss in the nanocomposites. The first one is caused by removal of water and small molecules, while the second one is because of the decomposition of MWCNT and PANI.
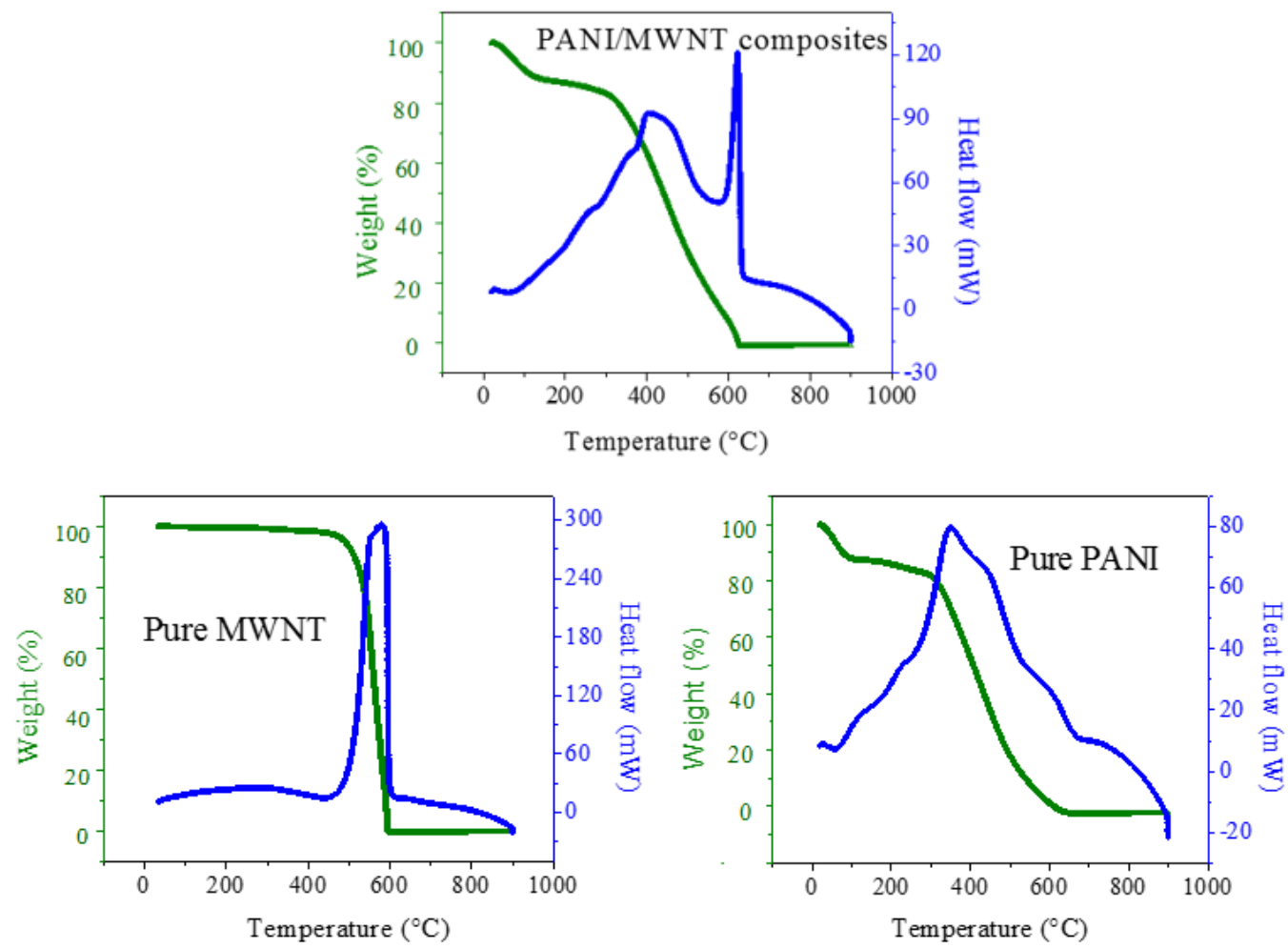

Figure 3.8 SDT curve for pristine MWCNT, pure PANI and PANI/22\% MWCNT composites films. 


\subsubsection{Damping Ratios Discussion}

Table 3.2 shown the damping property test results of PANI/MWCNT films. It shows that with the proportional increase of MWCNT in the PANI/MWCNT films, the damping ratio value increased, which means the PANI/44\% MWCNT films have a little bit higher of damping ratios than PANI/11\% MWCNT and PANI $/ 22 \%$ MWCNT. Also, when tests were performed with smaller scale of aluminum beam, the damping ratio value almost doubled. So, it indicates that the damping property of PANI/MWCNT composites films are related with the frequency of attached beams both through mass change and equivalent spring constant. However, with 33\% more of MWCNT added to the PANI/11\% MWCNT, the damping ratio increased only $10 \%$ for the 5.13 inch $\times$ linch $\times 0.12$ inch beam.

Table 3.2 Damping ratio values of PANI/MWCNT films in different components and different aluminum beam dimensions.

\begin{tabular}{|l|c|c|}
\hline & 5.13 inch $\times 1$ inch $\times 0.12$ inch & 2.4 inch $\times 1$ inch $\times 0.008$ inch \\
\hline PANI/11\% MWCNT & 0.00108 & 0.0020 \\
\hline PANI/22\% MWCNT & 0.00118 & 0.0023 \\
\hline PANI/44\% MWCNT & 0.0012 & broke \\
\hline
\end{tabular}

The data shown in Table 3.3 indicated that circular PANI/11\% MWCNT attached onto short aluminum beam ( $6 \mathrm{~cm}$ in length) displayed higher damping ratios value than any of the mid-length beam $(9.3 \mathrm{~cm}$ long $)$ and long beam in length of $12.6 \mathrm{~cm}$ when 
fixed on specimen side, \#. Also, the results showed the same trend, i.e., that short beam displays higher damping ratio values than middle and long beams when clipped on the free end $*$ sides. Furthermore, when the beam was clipped on \# side, it showed much higher damping values compared with the case of the beam clipped on * side of each sample. Circular PANI/11\% MWCNT composites achieved the highest damping ratio value to 0.00798 when clipped on \# side of short beams.

Table 3.3 Damping ratios of PANI/11\% MWCNT fixed on either the \# or * sides.

\begin{tabular}{|l|c|c|c|c|c|c|}
\hline \multirow{2}{*}{} & \multicolumn{2}{|c|}{ short beam } & \multicolumn{2}{c|}{ middle beam } & \multicolumn{2}{c|}{ long beam } \\
\hline & fix on \# & fix on * & fix on \# & fix on * & fix on \# & fix on * \\
\hline & 0.0084 & 0.0032 & 0.004 & 0.0031 & 0.0041 & 0.0022 \\
\hline & 0.0083 & 0.0035 & 0.0045 & 0.0031 & 0.0037 & 0.0024 \\
\hline & 0.0078 & 0.0038 & 0.004 & 0.0032 & 0.0034 & 0.0025 \\
\hline & 0.0075 & 0.0031 & 0.0044 & & 0.0035 & 0.0021 \\
\hline Average & 0.00798 & 0.00336 & 0.00428 & 0.003133 & 0.00372 & 0.0023 \\
\hline
\end{tabular}

\subsubsection{Strain Sensor Properties of PANI/11\% MWCNT Nanocomposites}

\subsubsection{Theory of Strain Sensor}

A strain gauge is an electrically conducting device where the resistance of the device is directly proportional to the strain imposed. The strain gauge is bonded to the 
specimen and the strain is transmitted from the specimen to the gauge. This type of transducer is widely used to determine the strain over a small area of a component subjected to a deflection. If a relationship between force and strain for the instrumented component can be found, a strain gauge transducer can be used to infer the value of an applied force.

The displacement of the tip of a cantilevered beam for a tip point load is known as:

$$
y=\frac{P L^{3}}{3 E I}
$$

where: $\mathrm{P}$ is an applied load at the tip of the beam.

$\mathrm{L}$ is the length from the support to the free end of the beam.

$\mathrm{E}$ is the modulus of elasticity of the beam.

I is the moment of initial of the beam, $I=b h^{3} / 12 . b$ is the width of the beam and $\mathrm{h}$ is the thickness of the beam.

The strain on the upper surface of the beam is given by the formula:

$$
\varepsilon=\frac{\Delta L}{L}=\frac{P L_{1} h}{2 E I}
$$

where: $\varepsilon$ is the strain on the upper surface of the beam.

$\Delta \mathrm{L}$ is the deformation on the upper surface of the beam.

$\mathrm{L}_{1}$ is the distance from the gauge to the load $\mathrm{P}$.

The relationship between change of the length and change in the resistance is:

$$
\frac{\Delta R}{R}=G \frac{\Delta L}{L}
$$

where: $\mathrm{R}$ and $\Delta \mathrm{R}$ are the resistance of the gauge and its change.

$\mathrm{G}$ is the gauge factor. 
The relationship between strain and output voltage in a quarter bridge circuit is:

$$
\varepsilon=\frac{4}{G} \frac{V_{0}}{V_{s}}
$$

Where: $V_{s}$ and $V_{o}$ are source and output voltages.

Herein, the dimension of the Aluminum beam is fixed, which the thickness $\mathrm{h}=0.3175$ $\mathrm{cm}$, width $\mathrm{b}=2.54 \mathrm{~cm}$. And the distance from the gauge to the load is fixed too. So, the deformation of the surface would be corresponding to a certain load $(2.4525 \mathrm{~N}, 4.4145$ $\mathrm{N}, 6.3765 \mathrm{~N}, 8.3385 \mathrm{~N}$ and $10.3005 \mathrm{~N}$ which converted by weight of $250 \mathrm{~g}, 450 \mathrm{~g}$, $650 \mathrm{~g}, 850 \mathrm{~g}$ and $1050 \mathrm{~g}$ ) which applied to the end of the beam. So, the strain change of the samples could be calculated for each load. Then, the V output was read directly from the Wheatstone bridge, leading to the strain voltage relationship.

\subsubsection{The Drift Test of PANI/MWCNT Sensor}

The drift test was executed for the sample and for the strain gage for a period of 30 minutes. Figure 3.9 shows typical drift test results for traditional foil strain gage and four sample sensors. We note that the traditional foil strain gauge remains stable, which shown in blue line in Figure 3.9 (f). Although both PANI/11\% MWCNT (series 2) and PANI/22\% MWCNT (series 3) showed stabilities, the light blue line pure PANI (series 1) and the yellow line PANI/44\% MWCNT (series 4) displayed a little more stability than both of PANI/11\% MWCNT and PANI/22\% MWCNT. Overall, for all these threedifferent composition nanocomposite, namely, PANI/11\% MWCNT, PANI/22\% MWCNT and PANI/44\% MWCNT, all showed small drift results during the half hour drift test. 


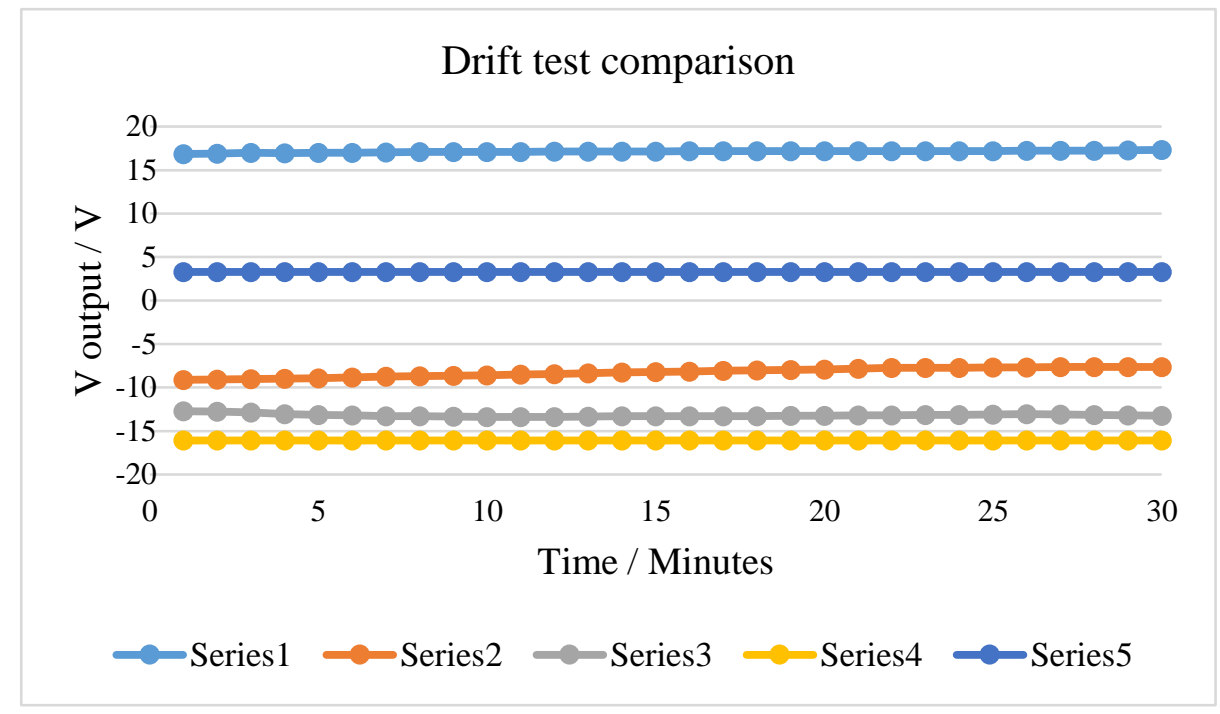

Figure 3.9 Drift test comparison of pure PANI, PANI/11\% MWCNT, PANI $/ 22 \%$ MWCNT, PANI $/ 44 \%$ MWCNT and strain gauge sensors. (a) series 1 is pure PANI, (b) series 2 is PANI/11\% MWCNT, (c) series 3 is PANI/22\% MWCNT, (d) series 4 is $\mathrm{PANI} / 44 \%$ MWCNT and (f) series 5 is traditional foil strain gauge.

\subsubsection{The Linearity of the PANI/MWCNT}

The environment such as temperature, humidity and so on can have effect on sensors performance. Here we compared sensors by sealing our samples from the environment by covering with a three thin layers of electrical non-conductive adhesive (glue). Table 3.4 shows a comparison between both PANI/22\% MWCNT and PANI/44\% MWCNT in the cases where the sample was isolated and not isolated from the environment. The electrical resistance of both PANI/22\% MWCNT and PANI $/ 44 \%$ MWCNT without glue upon them are $19 \Omega$ and $9 \Omega$, respectively, while those with glue are $27 \Omega$ and $15 \Omega$, respectively. It also showed that the more MWCNT was added in the composites, the lower of their electrical resistance $(\mathrm{R})$, which is consistent with result concluded in section 3.2. Also, both $\mathrm{R}$ increased when the samples were shielded from the environment by covering them with the glue. 
Table 3.4 The comparison of PANI/22\% MWCNT and PANI/44\% MWCNT when covered and without covered by a thin layer of glued to shield from the environment.

\begin{tabular}{|l|l|l|l|l|l|}
\hline & & \multicolumn{2}{|l|}{ PANI/22\% MWCNT } & \multicolumn{2}{l|}{ PANI/44\% MWCNT } \\
\hline Mass load/g & strain $(\varepsilon) / \mu \mathrm{m}$ & Vo1/mV & Vo1-glue/mV & Vo2 / mV & Vo2-glue/mV \\
\hline 250 & 132.63 & -13.218 & -10.036 & -16.095 & -16.074 \\
\hline 450 & 238.734 & -13.201 & -10.029 & -16.098 & -16.076 \\
\hline 650 & 344.838 & -13.194 & -10.018 & -16.099 & -16.077 \\
\hline 850 & 450.942 & -13.183 & -10.009 & -16.101 & -16.078 \\
\hline 1050 & 557.046 & -13.158 & -9.995 & -16.102 & -16.079 \\
\hline
\end{tabular}

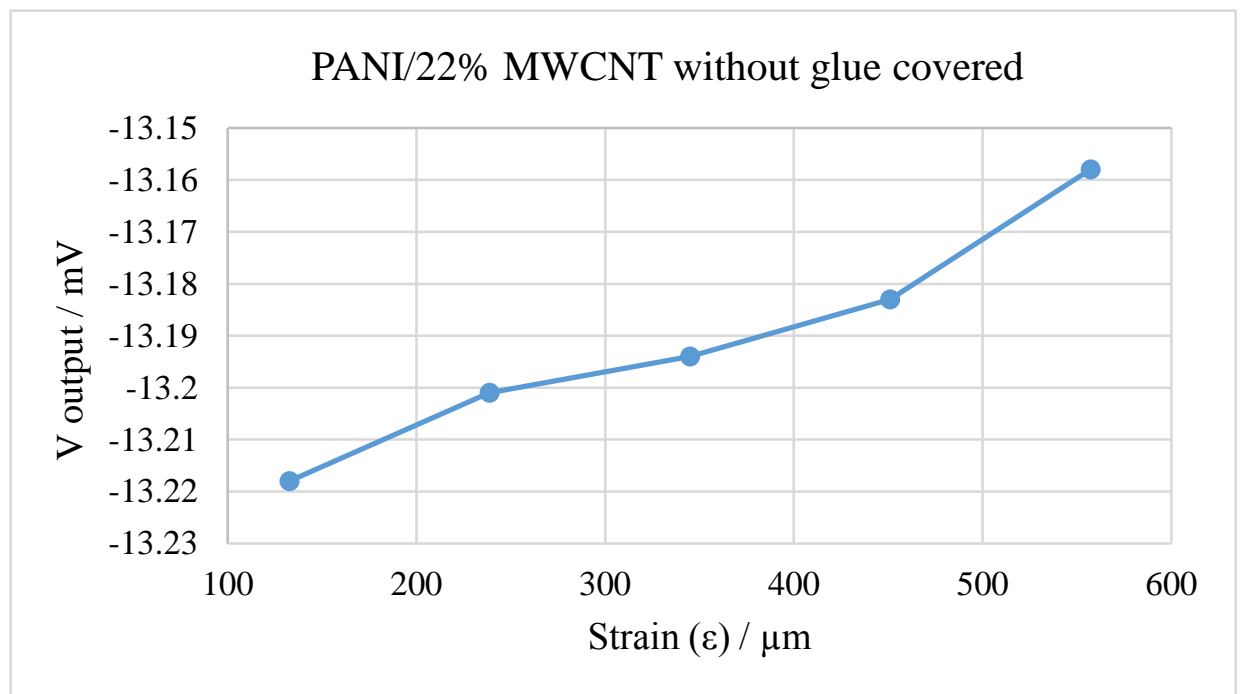

Figure 3.10 Strain sensor test of PANI/22\% MWCNT showing load vs. output.

Table 3.4 indicated that the differences for all those samples during the strain range $132.63 \mu \mathrm{m}$ to $557.046 \mu \mathrm{m}$ are slight. The biggest difference is $0.06 \mathrm{mV}$ when PANI/22\% MWCNT is not covered with glue. However, both PANI/22\% MWCNT and PANI/44\% MWCNT covered with glue showed more linearity than those that were not covered with the glue, (see Figures 3.10-3.13). 


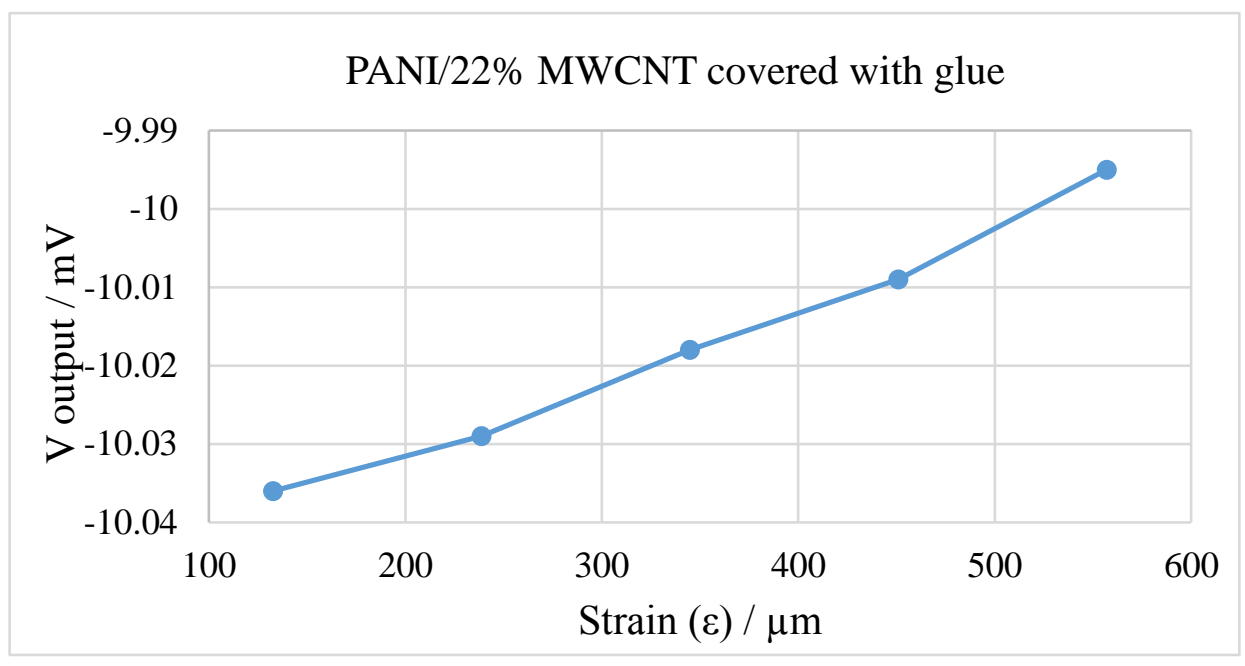

Figure 3.11 Strain sensor test of PANI/22\% MWCNT with glue showing load vs. output.

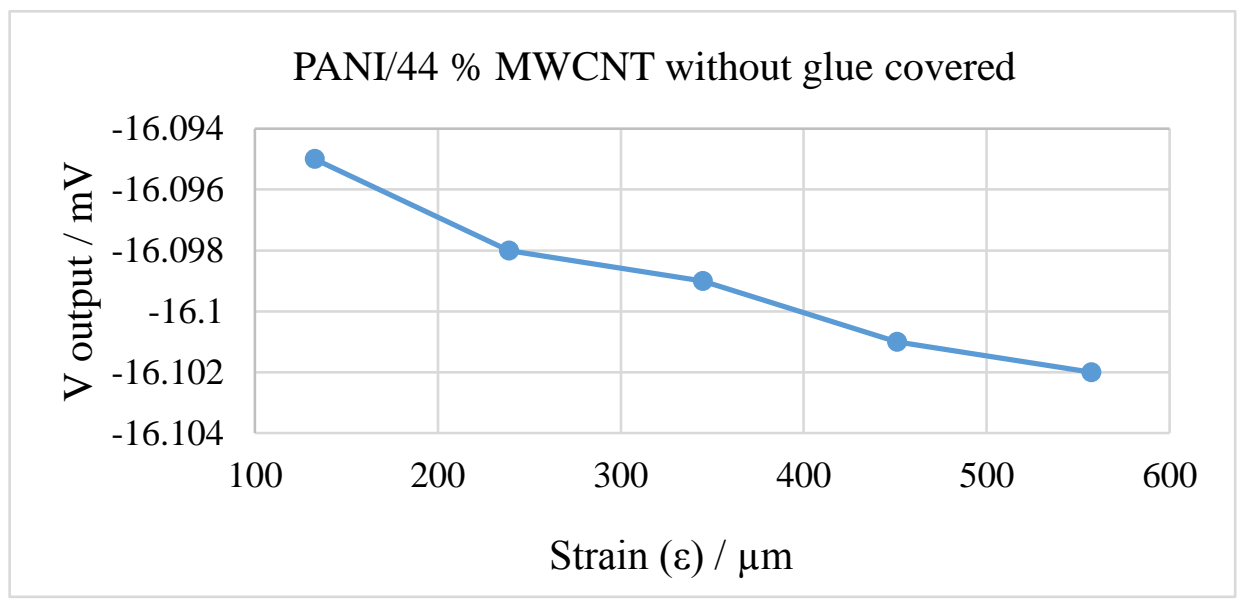

Figure 3.12 Strain sensor test of PANI/44\% MWCNT without glue covered showing load vs. output.

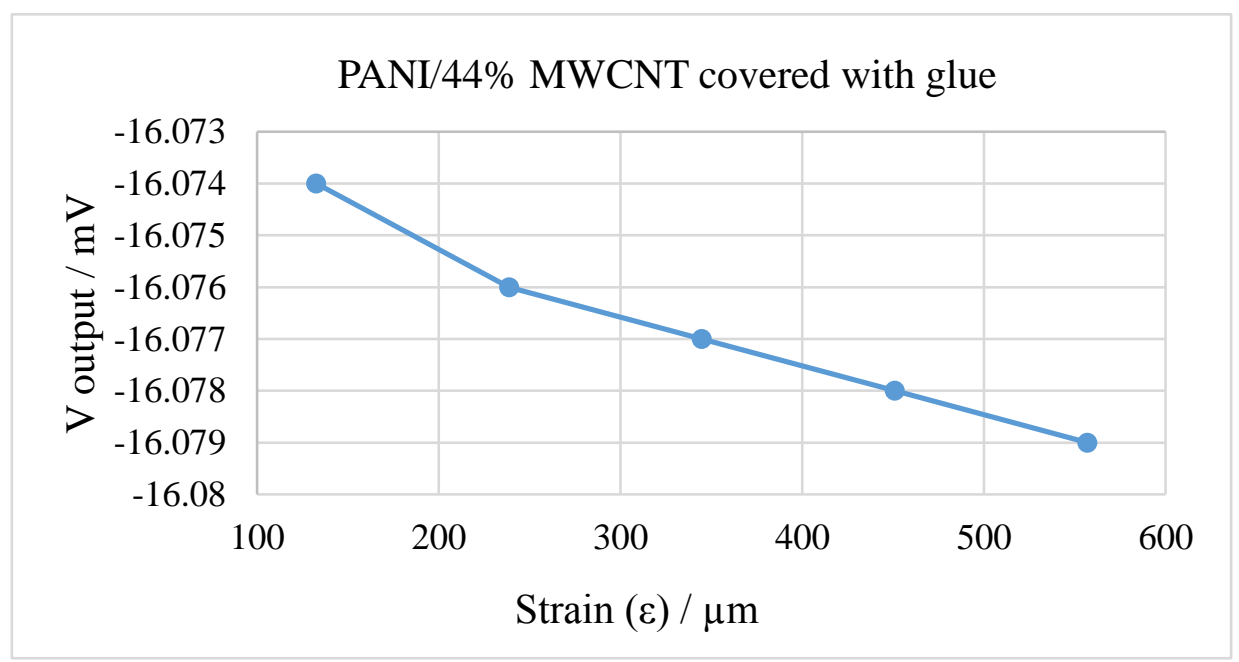

Figure 3.13 Strain sensor test of PANI/44\% MWCNT with glue showing load vs. output. 


\subsubsection{Morphological Characterization of the PANI/ MWCNT Film}

When one looks at the overall properties of the different PANI/MWCNT samples and what is to be accomplished, it is for this reason that the PANI/11\% MWCNT sample was concluded to be the best of the samples and why it was further tested for damping capabilities. Figure 3.14 provides the TEM images of pure MWCNT (Figure 3.14 (a)) and PANI/ 11\% MWCNT (Figure 3.14 (b)), which shows that the MWCNT and the PANI were integrated to form PANI/ 11\% MWCNT nanocomposites. The diameters of the MWCNT are about $15 \pm 5 \mathrm{~nm}$ (Figure 3.14 (a)) while the diameters of the PANI/11\% MWCNT are about $70 \mathrm{~nm}$ (Figure 3.14 (b)). It indicates that the MWCNT nanofibers were coated with a dense PANI layers. Furthermore, comparing both TEM images shown below, the walls of PANI/ 11\% MWCNT nanofibers were rougher than the walls of MWCNT nanofibers. It indicates that the polymerization of PANI was at least part of the nucleation growth and that the MWCNT was initially acting as the nucleating agent, which led to a new interwoven fibrous structure (Figure 3.15 (b)).
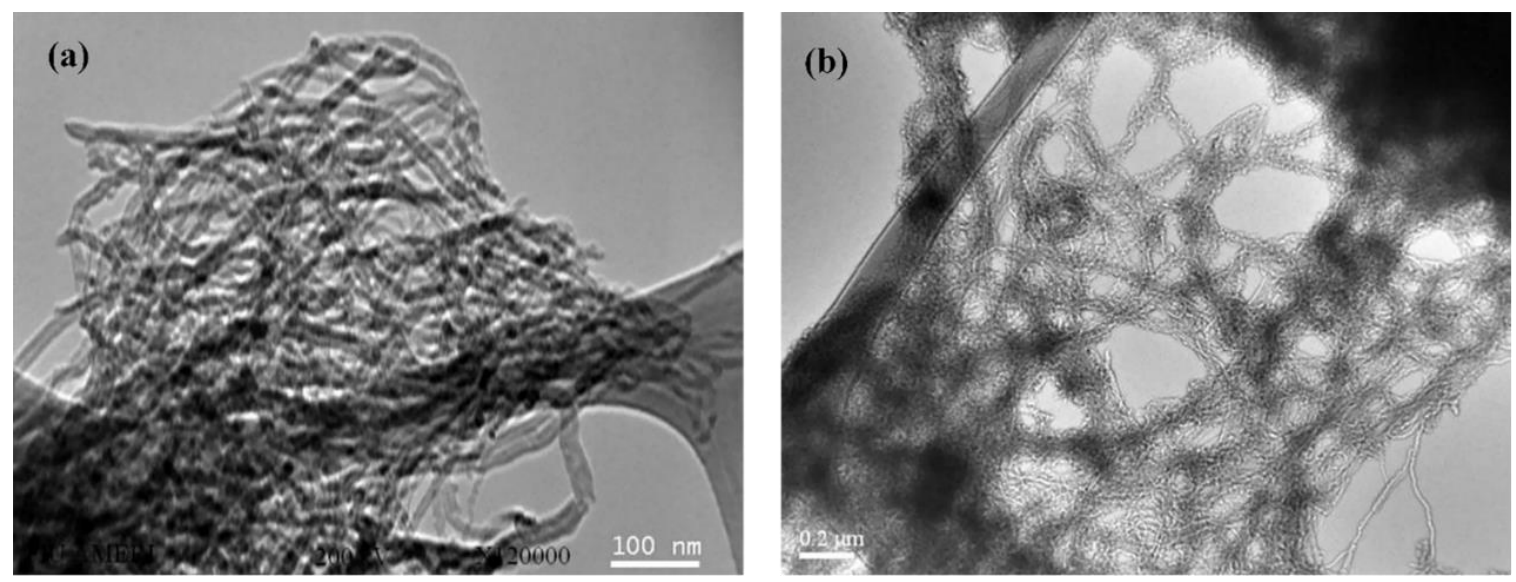

Figure 3.14 TEM images of (a) pure MWCNT and (b) PANI/11\% MWCNT. 

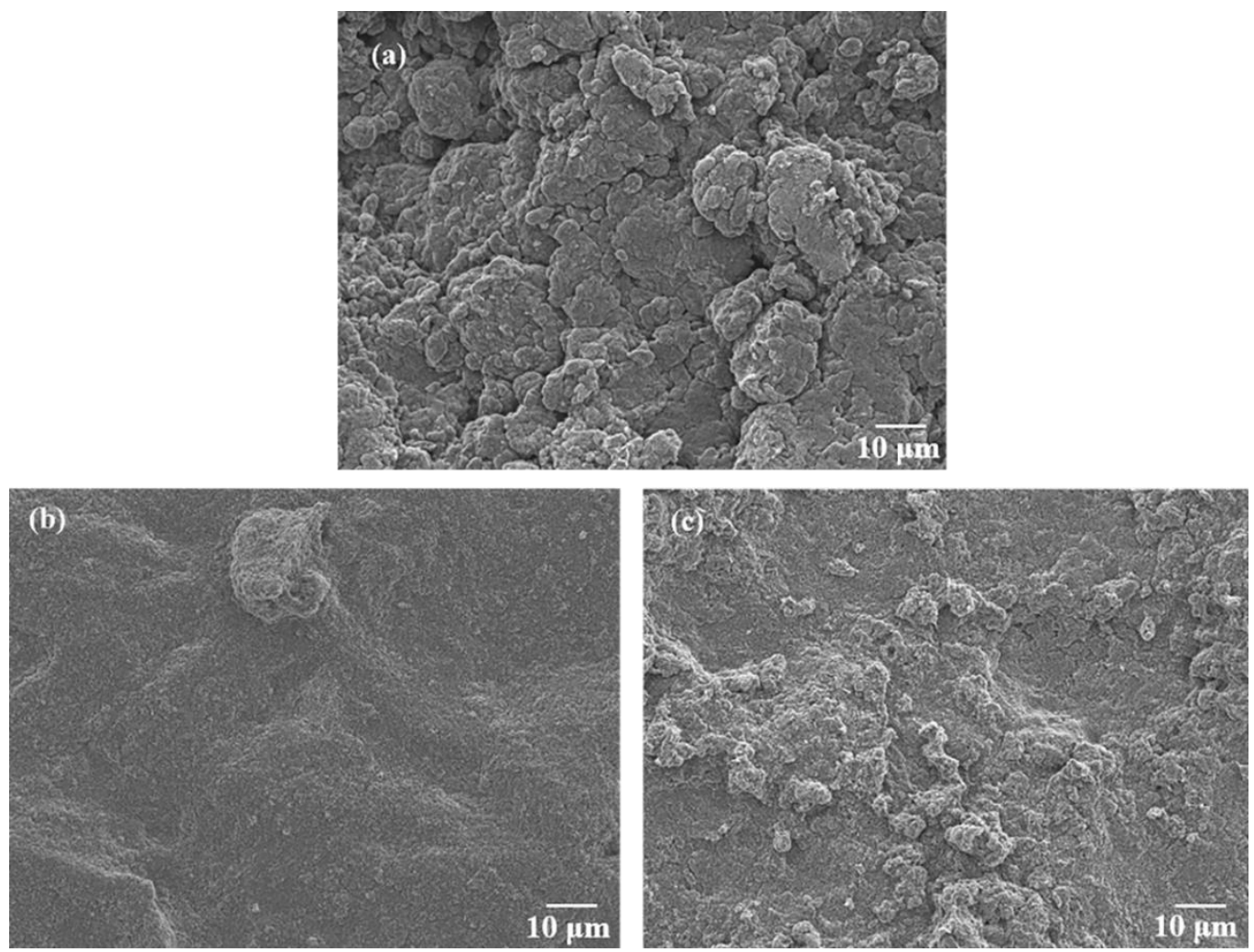

Figure 3.15 SEM image of (a) pure MWCNT, (b) pure PANI and (c) PANI/11\% MWCNT composite films.

Figure 3.15 is the SEM images of surfaces of pure MWCNT (Figure 3.15 (a)), pure PANI (Figure 3.15 (b)) and PANI/11\% MWCNT nanocomposites (Figure 3.15 (c)) films. It shows the different roughness of the three different films surfaces. The pure MWCNT have the roughest surface, while the PANI have the smoothest surface among three films surfaces. The roughness of the nanocomposites PANI/ 11\% MWCNT falls between the two, which means the order of the surfaces roughness of these three films are pure MWCNT $>$ PANI/11\% MWCNT nanocomposites $>$ pure PANI. Combined with the results of TEM results shown in Figure 3.9, it indicates that during the polymerization of PANI with the presence of MWCNT, most MWCNT have been entrapped into the PANI films. 


\subsection{Conclusion}

Different compositions of PANI/MWCNT nanocomposites were synthesized through an in-situ polymerization method. Frit compression method was introduced to make PANI/MWCNT nanocomposite films/discs. SEM and TEM results showed that this technique produced a coating of PANI onto the MWCNT (this implies a layer upon layer yet you say the CNT were well dispersed), which indicated that carbon nanotubes were well dispersed in the polymer matrix. The electrical resistance was largely reduced by introducing MWCNT into the PANI. The electrical resistance of the composites could be controlled by adding specific amounts of the MWCNT. Nanoindentation test results showed that the PANI greatly contributed to the increase in the Young's modulus and hardness of the PANI/22\% MWCNT nanocomposites films. The results of SDT test indicate that thermal stability of PANI $/ 22 \%$ MWCNT was improved by about $50{ }^{\circ} \mathrm{C}$.

Damping property test results of PANI/MWCNT films showed that with the proportional increase of MWCNT in the PANI/MWCNT films, the damping ratio value increased, which means the PANI/44\% MWCNT films have a little bit higher damping ratio than PANI/11\% MWCNT and PANI/22\% MWCNT samples. Also, when tests were performed with smaller scale aluminum beams, the damping ratio value almost doubled. However, with $33 \%$ more of MWCNT added to the PANI $/ 11 \%$ MWCNT, the damping ratio increased by only $10 \%$ for 5.13 inch $\times 1$ inch $\times 0.12$ inch beam.

Also, the shorter and the thinner the $\mathrm{Al}$ base, the higher the damping ratios for PANI/MWCNT when clamped on the same sides (free end side or sample side). Furthermore, the location of the clamped support of the cantilever beam while 
performing the free vibration damping tests was very important to the magnitude of the damping ratio. When the beam was clamped close to the sample end, it displayed higher damping ratios than when clamped to the free, uncovered end of the beam.

Last, both PANI $/ 22 \%$ MWCNT and PANI $/ 44 \%$ MWCNT covered with glue showed more linearity than those that were not covered with the glue. 


\section{CHAPTER IV}

\section{PREPARATION OF RECTANGULAR POLYANILINE/MULTIWALLED CARBON NANOTUBE AND CHARACTERIZATION OF THEIR STRUCTURAL DAMPING AND STRAIN SENSING PROPERTIES}

\subsection{Introduction}

The frit compression method is introduced to fabricate rectangular PANI/MWCNT nanocomposite films/discs. The reason we studied the rectangular PANI/MWCNT is that they can be considered one dimensional in form, which simplifies the derivation of displacement functions or to determine the modes of vibration we already have. The thickness of these films/discs can also be controlled. Four different compositions were synthesized and compared through their damping and strain sensing properties. Once the best composition was determined, namely PANI/11\% MWCNT samples, then different tests were conducted to further evaluate the damping and strain sensing properties.

\subsection{Experimental Sections}

\subsubsection{In Situ Synthesizing of Rectangular PANI/MWCNT Nanocomposites}

The MWCNT was treated employing the same procedure used in section 3.1 of Chapter III. $64.375 \pm 0.010 \mathrm{mg}$ of MWCNT (Newton, MA, www.nano-lab.com: PD15L5-20) with length of 5-20 $\mu \mathrm{m}$ and outer diameter of $15 \pm 5 \mathrm{~nm}$ were dispersed by sonication in $125 \mathrm{ml}$ of $1 \mathrm{M} \mathrm{HCl}$ for $30 \mathrm{~min}$. Then, $1.287 \pm 0.003 \mathrm{~g}$ of aniline was added and the mixture was sonicated for another $30 \mathrm{~min}$. After that, $1.027 \pm 0.003 \mathrm{~g}$ of ammonium persulphate (APS) dissolved in $125 \mathrm{ml}$ of $1 \mathrm{M} \mathrm{HCl}$ was added at once and polymerization was conducted at $0{ }^{\circ} \mathrm{C}$ with water-ice bath under sonication for $4 \mathrm{hrs}$. The precipitated products were washed 
with deionized water. Then the PANI/11\% wt. MWCNT composite was made. Three different ratios of PANI/MWCNT nanocomposites films listed in Table 4.1 were made. Pure PANI was made by not adding any MWCNT into it.

Table 4.1 Synthesizing different components of PANI/MWCNT composites and its control.

\begin{tabular}{|c|c|c|c|}
\hline & MWCNT (mg) & Aniline (g) & APS (g) \\
\hline Pure PANI & 0 & 1.445 & 1.163 \\
\hline PANI/11\% MWCNT & 64.375 & 1.287 & 1.027 \\
\hline PANI/22\% MWCNT & 128.75 & 1.129 & 0.903 \\
\hline PANI/44\% MWCNT & 257.50 & 0.813 & 0.643 \\
\hline Pure MWCNT & 513.06 & 0 & 0 \\
\hline
\end{tabular}

$454.54 \pm 0.005 \mathrm{mg}$ of obtained PANI/MWCNT suspension was transferred into a specially built $50 \mathrm{ml}$ rectangular mold (see Figure 4.1), which was equipped with frits with a pore diameter of $70 \mu \mathrm{m}$ (frits purchased from Sigma-Aldrich). To remove the solvent, two different compression forces of $289 \pm 3 \% \mathrm{~N}$ and $334 \pm 3 \% \mathrm{~N}$ were applied to the plungers, which were measured by FlexiForce adapter (See Figure 4.2).

After the solvent was squeezed out, the frits-PANI/MWCNT sandwich with the rectangular mold was dried at $110{ }^{\circ} \mathrm{C}$ in an oven for $12 \mathrm{hrs}$. Three different component PANI/MWCNT composite film and one pure PANI were obtained after removing it from the rectangular mold.

The pure raw rectangular MWCNT film was prepared by the same frit compression procedure. $454.54 \pm 0.005 \mathrm{mg}$ MWCNT was suspended into $3 \mathrm{ml}$ deionized water, then the mixture was simply transferred into the cuboid mold to squeeze out the solvent. After 
drying at $110^{\circ} \mathrm{C}$ in an oven for $12 \mathrm{hrs}$., the MWCNT was obtained by removing it from the rectangular mold.
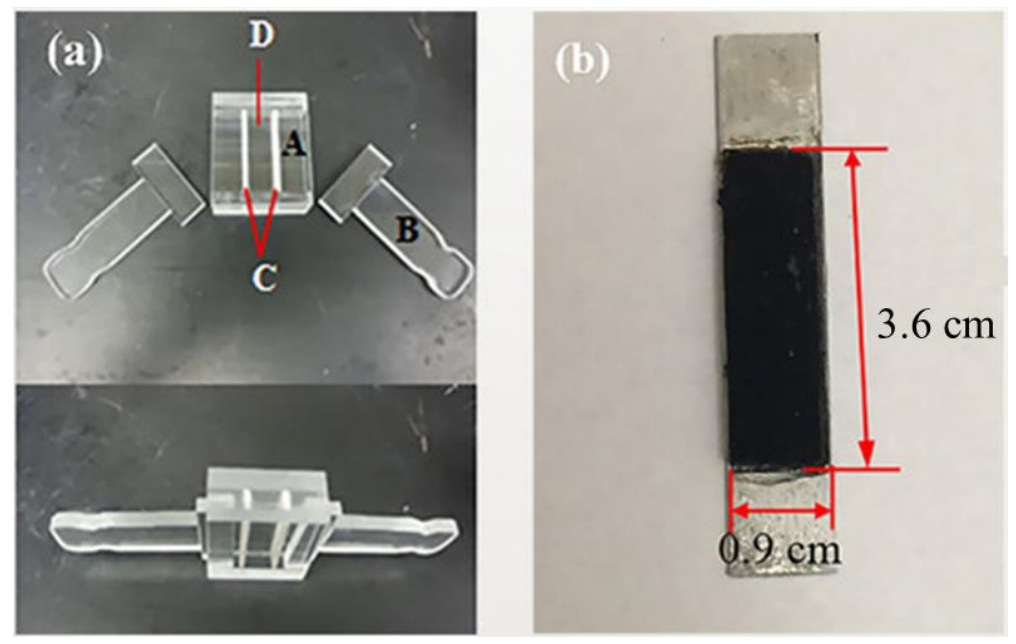

Figure 4.1 The frit compression system for making PANI/MWCNT nanocomposite films. (a) The frit compression system, (b) are the photo image of PANI/MWCNT nanocomposites. Inserts are A: cuboid; B: plungers; $\mathrm{C}$ : polypropylene frits and D: composites suspension.

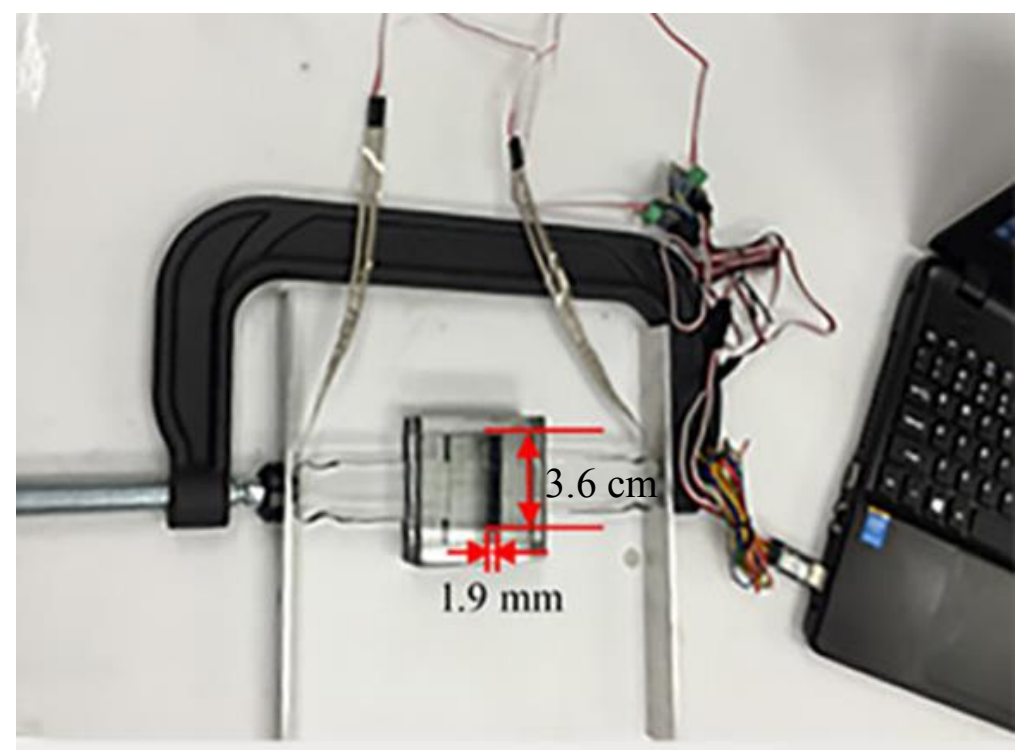

Figure 4.2 Schematic image of $289 \mathrm{~N}$ and $334 \mathrm{~N}$ force applied to plungers measured by FlexiForce adapter. The adapter's load measurements have a $\pm 3 \%$ error. 


\subsubsection{Characterization}

Transmission electron microscopy (TEM) and scanning electron microscopy (SEM) were used to determine their morphology properties. Free end vibration test method was adopted to study the damping properties of these samples. There were two different setups used to test the damping properties of the PANI/MWCNT nanocomposites: one was used to test the effect of sample size and location on the vibration damping of the beam; and, one was used to test how the sample's vibration damping capability was affected by single or double coverage of the base Al beam. Strain sensing properties were also studied.

\subsubsection{Specimen Setup for Damping Test Purpose}

Specimen for test 1: The polymer compositions of the samples used here were pure PANI, PANI $/ 11 \%$ MWCNT, PANI $/ 22 \%$ MWCNT, PANI $/ 44 \%$ MWCNT and pure MWCNT. The dimensions of the samples here is $3.6 \mathrm{~cm} \times 0.8 \mathrm{~cm} \times 1.9 \mathrm{~mm}$, which were bonded to the $\mathrm{Al}$ beams with dimension of $6 \mathrm{~cm} \times 2.5 \mathrm{~cm} \times 0.013 \mathrm{~cm}$ (Alloy 6061 aluminum, bought from MSC Industrial Co.) by nonconductive 3M CA100 liquid instant adhesive (See Figure 4.3 (a)). Three thin layers of 3M CA100 liquid instant adhesive were used to cover these samples to separate the samples from the environment. Each specimen was dried at room temperature for $24 \mathrm{hrs}$. Results from $\mathrm{Li}$ et al (Li et al., 2008) indicated that vibration related properties were affected by exposure to the environment.

Free vibration comparisons were made by using either the sample side, \#, or the uncovered side, called free end side *, alternately, as the fixed support for the cantilever beam. The red lines shown in Figure 4.3 (b) defined the locations where the beam was clamped. The unclamped side was then made to vibrate and a parameter called the damping ratio was determined. 


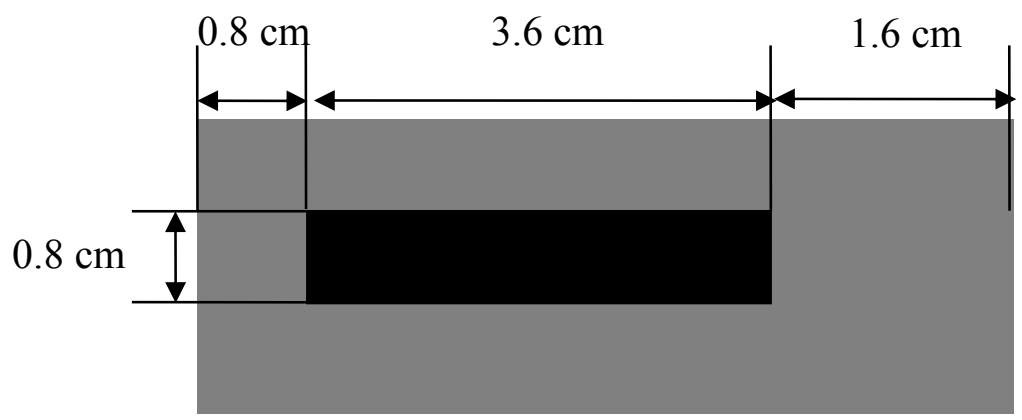

(a)

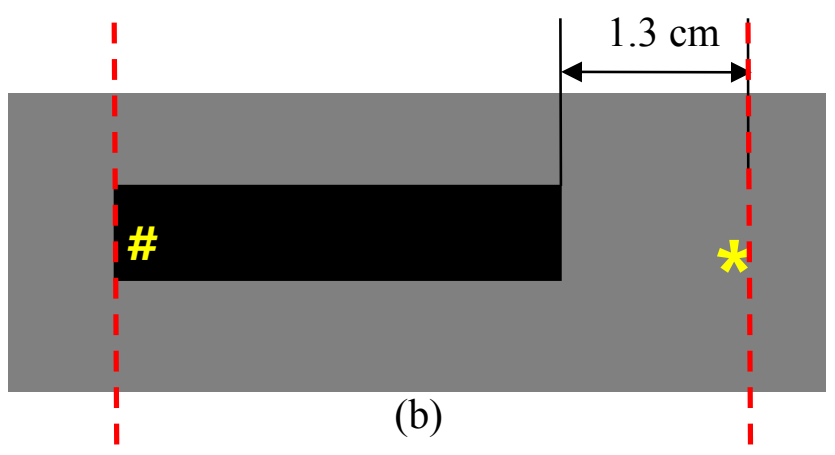

Figure 4.3 (a) Size of samples, Al beams and the locations where samples were attached. (b) Locations of sample side \# and free end side * where the beam was clamped.

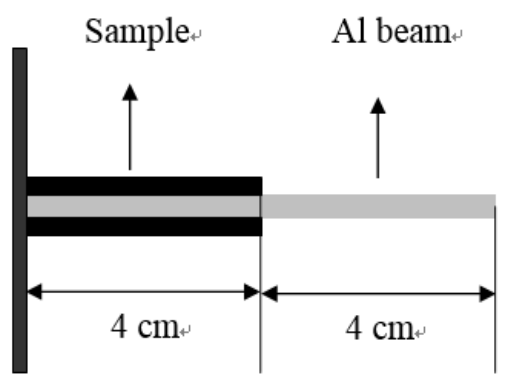

(a)

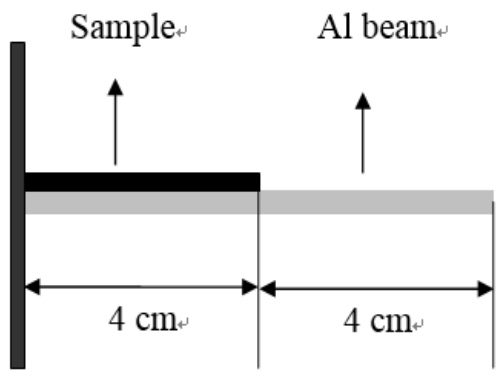

(b)

Figure 4.4 (a) PANI/11\% MWCNT sandwiched Al base beam. (b) PANI/11\% MWCNT attached one side of Al base beam.

Specimen for test 2: Two Aluminum (Al) beams were used with thickness of $2.4 \mathrm{~mm}$ and width of $0.9 \mathrm{~cm}$. Samples of $4 \mathrm{~cm} \times 0.9 \mathrm{~cm} \times 1.9 \mathrm{~mm}$ PANI $/ 11 \%$ MWCNT were 
attached the $\mathrm{Al}$ base beam, one beam with single coverage and one beam with both sides covered for comparison (See Figure 4.4). Three thin layers of 3M CA100 liquid instant adhesive were used to cover these samples to separate the samples from the environment. Each specimen was dried at room temperature for $24 \mathrm{hrs}$. The unclamped side was then made to vibrate and the damping ratio was determined.

Specimen for test 3: Two different thicknesses of $\mathrm{Al}$ beams were used to evaluate thickness effects. One is thin beam with $1.25 \mathrm{~mm}$ thickness and the other is of $2.4 \mathrm{~mm}$ thickness. The width of the beam used was $0.9 \mathrm{~cm}$ for both beams. The dimension of the PANI/ $11 \%$ MWCNT sample were $0.8 \mathrm{~cm} \times 4 \mathrm{~cm} \times 1.9 \mathrm{~mm}$, which was glued onto the Al beams by 3M CA100 liquid instant adhesive. Three very thin layers of adhesive were used to cover these samples to separate the samples from the environment. Each specimen was dried in room temperature for $24 \mathrm{hrs}$.

Table 4.2 Different thickness and distance from the clamp and the samples were tested.

\begin{tabular}{|c|c|c|c|c|}
\hline Al thickness & \multicolumn{4}{|c|}{ Distance from the clamp to sample (cm) } \\
\hline $2.4 \mathrm{~mm}$ & 0 & 1.7 & 5.4 & 7.7 \\
\hline $1.25 \mathrm{~mm}$ & 0 & 1.7 & 5.4 & 7.7 \\
\hline
\end{tabular}

For each thickness beam, several tests were conducted where the clamped edge was placed at different distances $(0 \mathrm{~cm}, 1.7 \mathrm{~cm}, 5.4 \mathrm{~cm}$ and $7.7 \mathrm{~cm})$, which correspond to redline marked as a, b, c and d (See Table 2.2 and Figure 4.5). The unclamped side was then made to vibrate and the damping ratio was determined. 


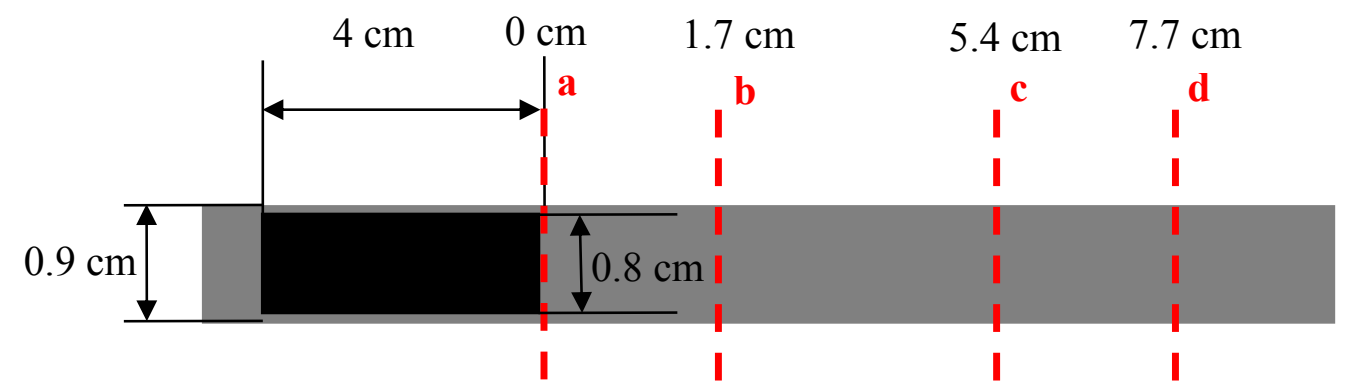

Figure 4.5 Dimension of samples and locations (red lines) where the clips clamped on (a) $0 \mathrm{~cm}$, (b) $1.7 \mathrm{~cm}$, (c) $5.4 \mathrm{~cm}$ and (d) $7.7 \mathrm{~cm}$ away to the samples. The dimension of the sample is $4 \mathrm{~cm} \times 0.8 \mathrm{~cm} \times 1.9 \mathrm{~mm}$. The width of the Al beam is $0.9 \mathrm{~cm}$.

Specimen for test 4 - Single side attachment: To determine if the "squeezing" load influenced the sensor properties when squeezing out the solvent, several levels of loading were used. We report on the two cases of $289 \mathrm{~N}$ and $334 \mathrm{~N}$ applied force, respectively. The force is applied via a FlexiForce adapter. The PANI/11\% MWCNT with dimension of $3 \mathrm{~cm}$ $\times 0.9 \mathrm{~cm} \times 1.9 \mathrm{~mm}$ were cut and attached to a $20.5 \mathrm{~cm} \times 0.9 \mathrm{~cm} \times 1.25 \mathrm{~mm}$ Aluminum base beam on one side (see Figure 4.6) using a very thin layer of glue. The specimen was dried at room temperature for $24 \mathrm{hrs}$. The unclamped side was made to vibrate and the damping ratio was determined for various lengths of the beam. The sample size did not change. Free vibration comparisons were made by using either the sample side, \#, or the uncovered side, *, alternately, as the fixed support for the cantilever beam. The red dashed lines shown in Figure 4.6 defined the locations where the beam was clamped. The side of the Al base beam that was without the sample was cut and the beam was clamped. The unclamped side was then made to vibrate and a parameter called the damping ratio was determined. This process was repeated after cutting the beam $1 \mathrm{~cm}$ at a time, until the cut end was next to the sample. The damping ratios then were compared. 


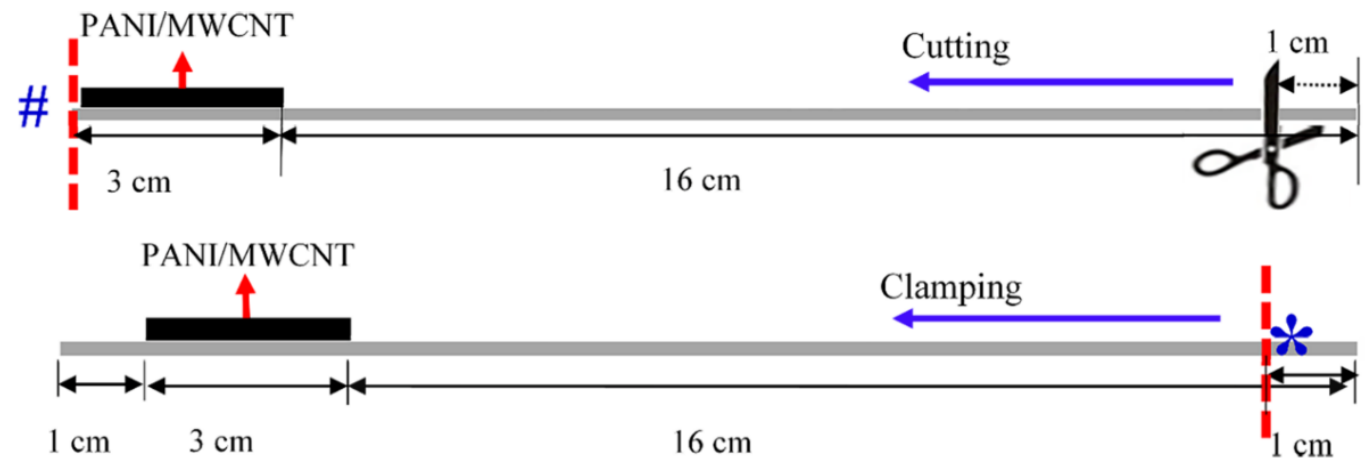

Figure 4.6 Schematic images of single side's attachment. The dimension of samples, Al beams and the locations where samples were attached, the locations of sample side \# and free end side * where the beam was clamped and the directions and lengths for both cutting and clamping.

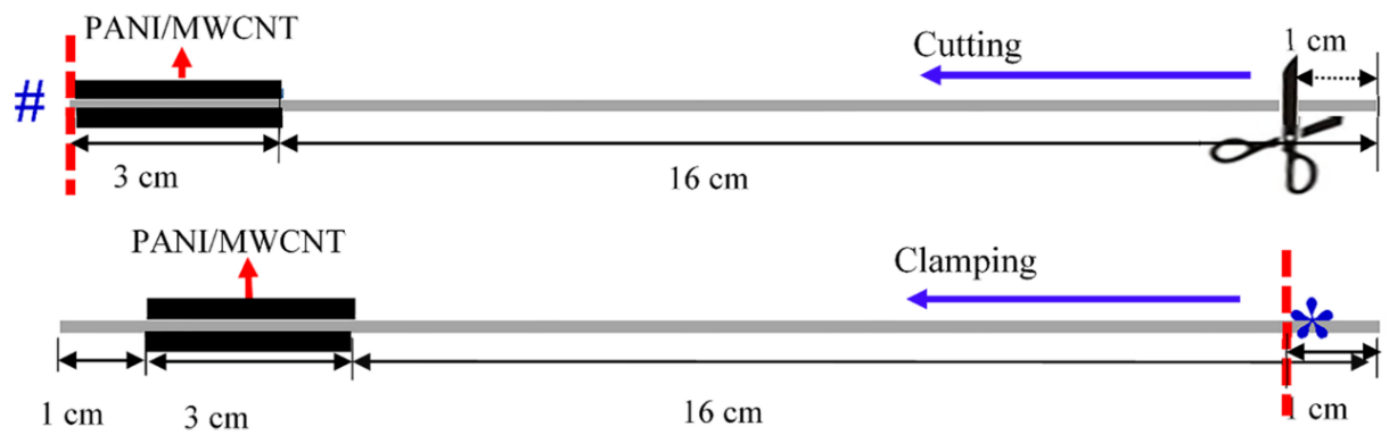

Figure 4.7 Schematic images for sandwich attachment. The dimension of samples, Al beams and the locations where samples were attached, the locations of sample side \# and free end side * where the beam was clamped and the directions and lengths for both cutting and clamping.

Specimen for test 5 - "Sandwich" attachment: The sample used in this experiment is with $334 \mathrm{~N}$ applied "squeezing" force on the PANI/11\% MWCNT. All the dimensions of sample and aluminum base beams, and the methodologies of testing applied to the single side are the same. The only change is that the Aluminum beam was "sandwiched" on both sides with the sample (see Figure 4.7). 


\subsubsection{Damping Test Methodology}

The cantilever beams of Figure 4.3-4.7 were given the same, small initial displacement and then released for free vibration. Free end displacements were measured by a laser vibrometer and a two channels digital oscilloscope was used display and store the signal. Five trials were made for each setup and the mean value of the damping ratio was calculated. This ratio can be related to the logarithmic decrement of consecutive maxima of end displacements and can be correlated to how much vibration energy is removed by the sample. The higher the damping ratio, the better the sample acts as a damper.

\subsubsection{Specimen Setup for Strain Sensing Test Purpose}

\subsubsection{Comparison of Different Components of PANI/MWCNT Nanocomposites}

The theory of the strain sensor is the same as described in section 3.3.5.1 of Chapter III. The components of PANI/MWCNT nanocomposites with dimension of $0.8 \mathrm{~cm} \times 4 \mathrm{~cm}$ $\times 0.19 \mathrm{~cm}$ were attached onto the $26.67 \mathrm{~cm} \times 2.54 \mathrm{~cm} \times 0.3175 \mathrm{~cm}$ Al beam. The sample attached to the $\mathrm{Al}$ beam were sealed with a three-thin layer of electrical non-conductive adhesive (glue). The Al beam was fixed on the lab table. The wires from the sample were connected to the Wheatstone bridge box. Loads of $2.453 \mathrm{~N}, 4.415 \mathrm{~N}, 6.377 \mathrm{~N}, 8.339 \mathrm{~N}$ and $10.301 \mathrm{~N}$, which are the equivalent weights for $250 \mathrm{~g}, 450 \mathrm{~g}, 650 \mathrm{~g}, 850 \mathrm{~g}$ and $1050 \mathrm{~g}$, were applied to the end of the aluminum beam, which led to the deformation of the beam and the sample attached onto it. The strain change of the samples could be calculated for each load. Finally, the data were recorded for each load and voltage output, V, read directly from the Wheatstone bridge. 

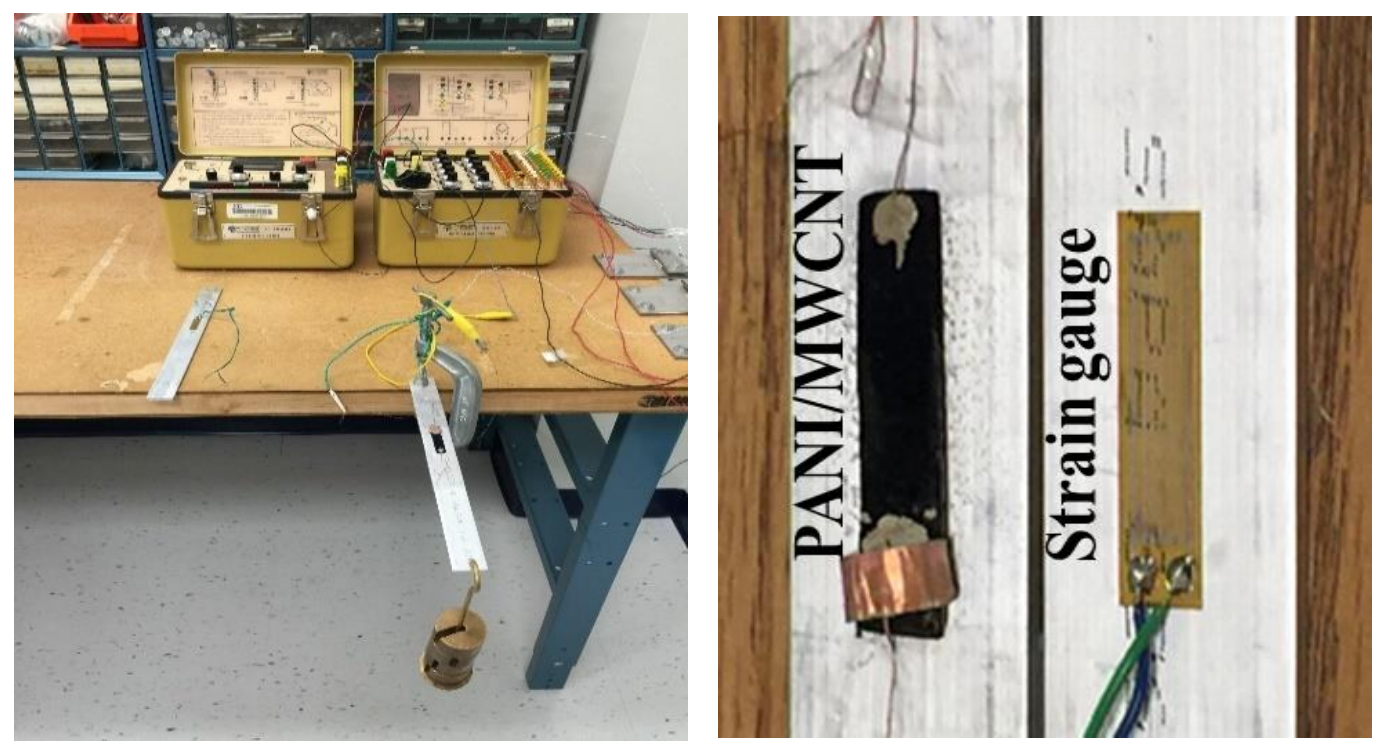

Figure 4.8 Strain sensor test setup.

\subsubsection{Sample Setup for Drift, Static Tensile Test for the PANI/11\%MWCNT}

The sensor was bonded to the center of a $1.55 \mathrm{~mm}$ thick 6061-T6 Aluminum rectangular bar using 3M CA100 liquid instant adhesive. Each specimen was held in place by a weight of $1.963 \mathrm{~N}$, which is equivalent to a mass of $200 \mathrm{~g}$, and dried at room temperature for $24 \mathrm{hrs}$. The bonding area was prepared to ensure a smooth surface, as is normally defined for a foil strain gage, with no oxide formations on the surface. A foil strain gage, Micro-Measurements N2A-00-10CBE-350, $3 \mathrm{~cm}$ long x $0.3 \mathrm{~cm}$ wide, was bonded on the back side of the Al beam at the same location as the sensor sample. $24 \mathrm{AWG}$ solid wire was used to connect decade resistor boxes manufactured by Eisco Labs in a Wheatstone bridge setup for the sensor or the foil strain gage. Prior to testing, resistance of the sensor and strain gage was measured and the corresponding decade resistance boxes were set to match. The decade resistor boxes have a resolution of about $1 \mathrm{ohm}$. This caused the initial millivolt potential difference to rarely start at zero. To compensate for this, where 
appropriate, start points were shifted to allow for comparison of different sensors. Constant voltage was provided during testing by $\mathrm{B} \& \mathrm{~K}$ precision power supply (1745A). The voltage supply was set to 5 volts for measured resistance above 20 ohms. For resistance below 20 ohms, the voltage supply was set to 2 volts. Signals for the drift and strain measurement tests were collected using National Instruments NI cDAQ-9172 board and LabView software. Linearity and sensitivity of the sensor were evaluated using quasi-static loadings. These loadings were carried out using a United SSTM-2K model tensile test machine.

\subsubsection{Drift Test Procedures for the PANI/11\% MWCNT Sensor}

The mounted sample was connected to the measurement system and placed in a no load and ambient temperature condition for at least 24 hours prior to testing. The test equipment was then turned on, and at least 60 minutes of data were collected for each sample. The drift test was performed several times for the sake of repeatability. The results were collected.

\subsubsection{Static Test Procedures for the PANI/11\% MWCNT Sensor}

The mounted sample was placed in the tensile testing machine and connected to the measurement system. The system was left idle for at least 1 hour prior to testing to remove any accumulated stresses due to mounting. Signal collection was initiated. The tensile test system was set to extend at a rate of $0.00254 \mathrm{~cm}+/-0.00127 \mathrm{~cm}$ per minute. This way the system was operating quasi-statically. The system was set to increase ramp force at the set extension rate until $1557 \mathrm{~N}$ of force was achieved. The system was then set to instantaneously reduce load to $0 \mathrm{~N}$. This procedure was repeated multiple times to determine repeatability of the results and hysteresis in the system. 


\subsubsection{Results and Discussion}

\subsubsection{Morphological Characterization of the PANI/22\% MWCNT Film}

SEM images were obtained to determine how well the MWCNT and the PANI were dispersed to form a homogeneous composite. SEM images of pure MWCNT, pure PANI and PANI/22\% MWCNT nanocomposites films are given in Figure 4.9. Compared with the random distribution of MWCNT, which displayed in Figure 4.9 (a) and (a'), the surface of pure PANI was much smoother (Figure 4.9 (b) and (b')). The surface smoothness of PANI/22\% MWCNT nanocomposite films is characterized to be between the pure PANI and pure MWCNT, which indicated that while polymerization of PANI with the presence of MWCNT, most MWCNT have been entrapped into the PANI films. Furthermore, the PANI/MWCNT nanocomposites showed the homogeneous coating of PANI onto the MWCNT indicating that carbon nanotubes were well dispersed in the polymer matrix, which is consistent with the TEM test results shown in Figure 4.10.

TEM images were obtained to determine how well the MWCNT and the PANI were integrated to form a homogeneous composite. TEM images of pure MWCNT and PANI/22\% MWCNT are shown in Figure 4.10. The diameter of the PANI/22\% MWCNT are about 70 $\mathrm{nm}$ (Figure 4.10 (b)) while the diameter of the MWCNT are about 10-20 nm (Figure 4.10 (a)). It appears that the MWCNT nanofibers were coated with a dense PANI layer (see Figure 4.10 (a)). Furthermore, these TEM images clearly displayed that the walls of PANI/MWCNT nanofibers were rougher than walls of MWCNT nanofibers. It indicated that the polymerization of PANI was at least part of the nucleation growth and the MWCNT was acting like a nucleating agent, which led to a new interwoven fibrous structure (Figure $4.10(b))$. 

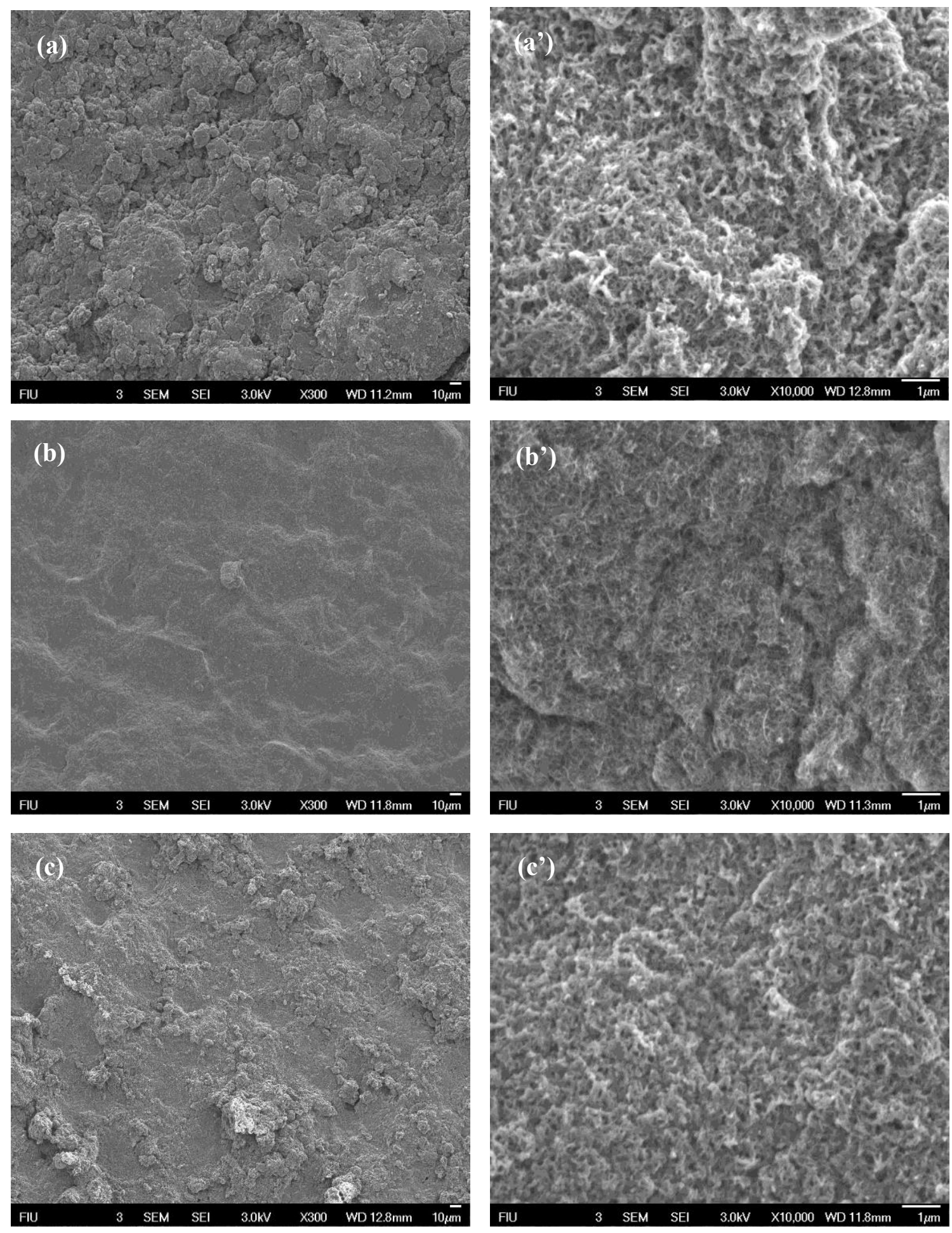

Figure 4.9 SEM image of (a) pure MWCNT, (b) pure PANI and (c) PANI/22\% MWCNT. 

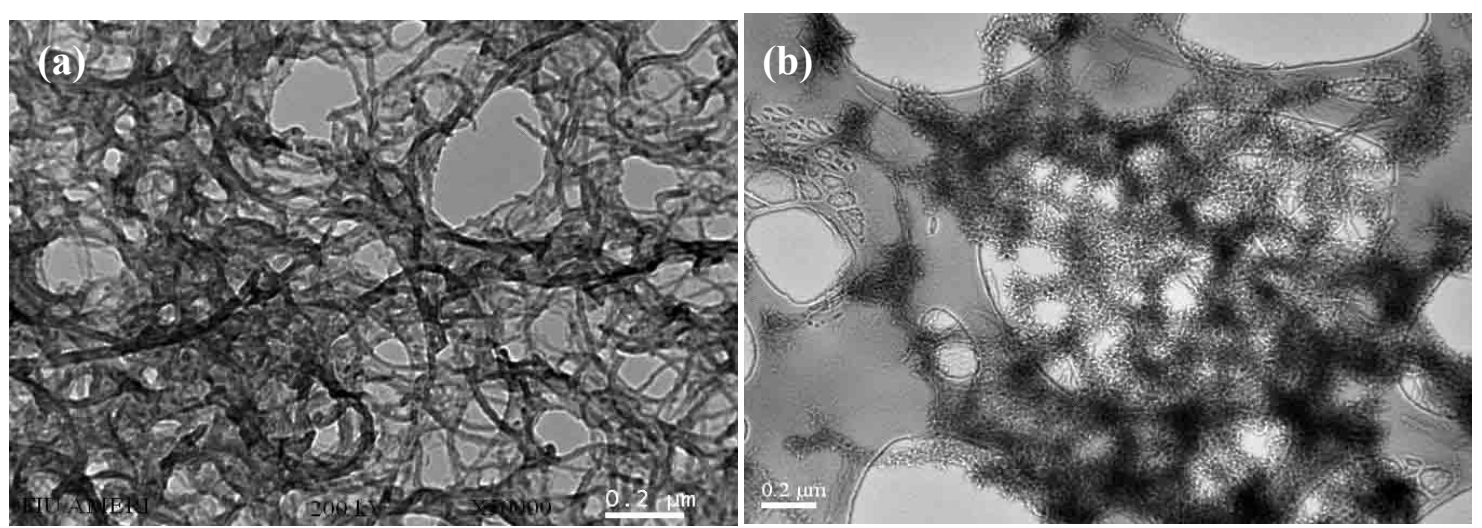

Figure 4.10 TEM images of (a) pure MWCNT and (b) PANI/22\% MWCNT nanocomposite films. (a'), (b') and (c') were higher magnification $(\times 10,000)$ images of $(a),(b)$ and (c), respectively.

\subsubsection{Damping Ratios Comparison of Different Component PANI/MWCNT}

As mentioned previously, the reason for the creation of the composite is for use as a sensor-actuator with damping capabilities. To check on the damping capability aspect, several tests were performed. For the first test, free vibration trial results are shown in Figure 4.11 .

Five trials were run for each of the different composites listed. The tests indicated that the PANI/11\% MWCNT composite displayed higher damping ratios than pure PANI, PANI $/ 22 \%$ MWCNT, PANI/44\% MWCNT and pure MWCNT when clamped either close to the uncovered $*$ side or close to the sample \# side (see Figure 4.3 for locations). The damping ratio of PANI/11\% MWCNT was 1.8 times larger than that of pure PANI and 1.35 times higher than PANI/22\% MWCNT when clamped on the uncovered* side. However, the damping ratio of PANI/11\% MWCNT was double that of the pure PANI and 1.43 times more than PANI/22\% MWCNT when clamped on the sample \# side. Also, when clamped on the sample \# side, the damping values were 2.2 to 3.1 times higher for each composite 
when compared to being clamped on the uncovered $*$ side. As the standard deviation was small in all cases, the results were deemed to be statistically significant.

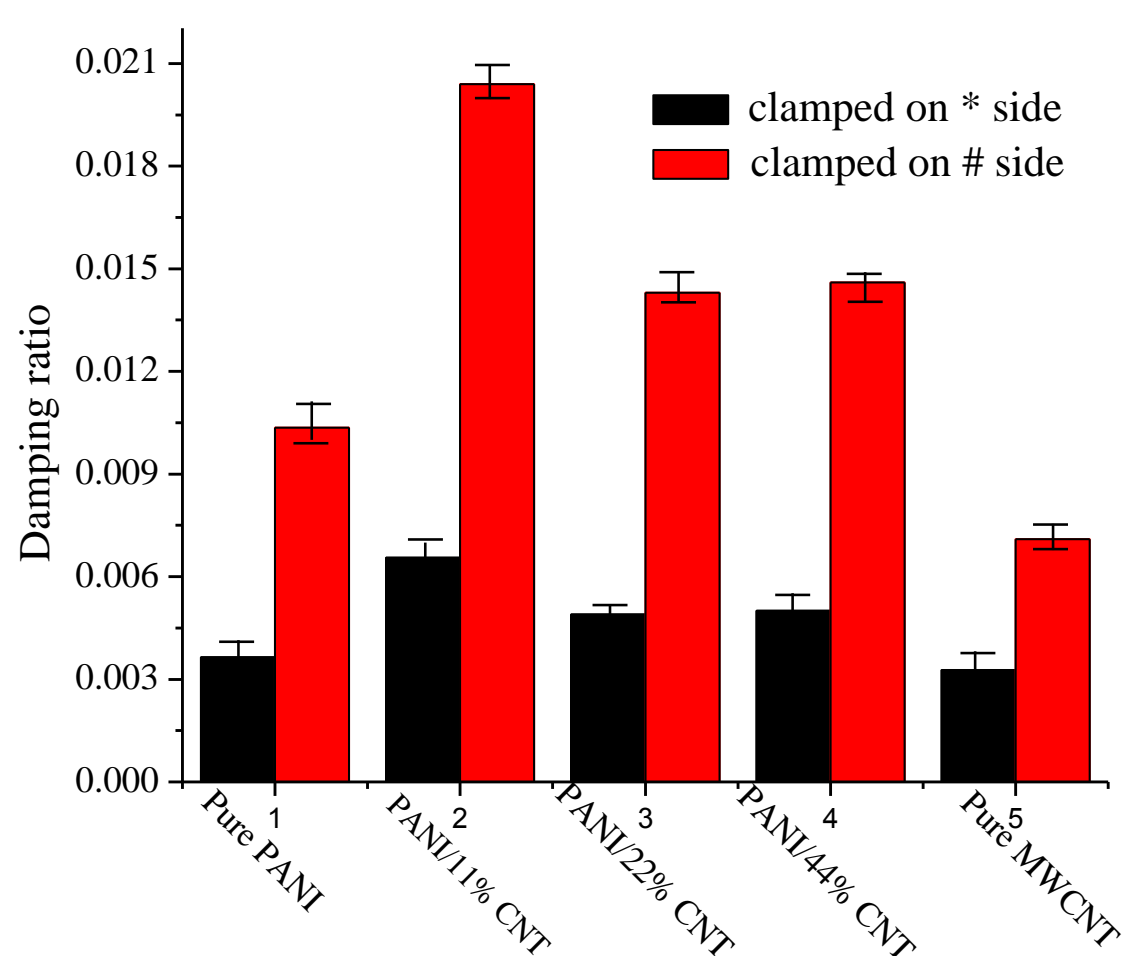

Figure 4.11 Damping ratios and standard deviation of rectangular pure PANI, PANI/11\% MWCNT, PANI $/ 22 \%$ MWCNT, PANI $/ 44 \%$ MWCNT and pure MWCNT clamped on the uncovered * side and sample \# side.

The damping ratio of PANI $11 \%$ MWCNT composite was 0.00656 when clamped on the uncovered * side; however, it reached a value of 0.0204 when clamped to the sample \# side. Thus, it indicated that where the samples were placed on the base beams played an important role in the damping results. Further, all PANI/MWCNT combinations had damping ratios that were much better than pure MWCNT alone. Our MWCNT damping ratio results were similar to those found by Li and Levy (Li and Levy, 2007a). 


\subsubsection{Comparison Single Side and Both Sides' Attachment of PANI/MWCNT}

For the second test (see Figure 4.4 for the configuration of the test setup), free vibration test results showed that the highest damping ratio of the PANI/11\% MWCNT sample attached to only one side of Al base beam was 0.00382 . However, the highest damping ratio achieved for the double-sided coverage reached a value of 0.072 , which is 18.85 times higher than for the single sided coverage.

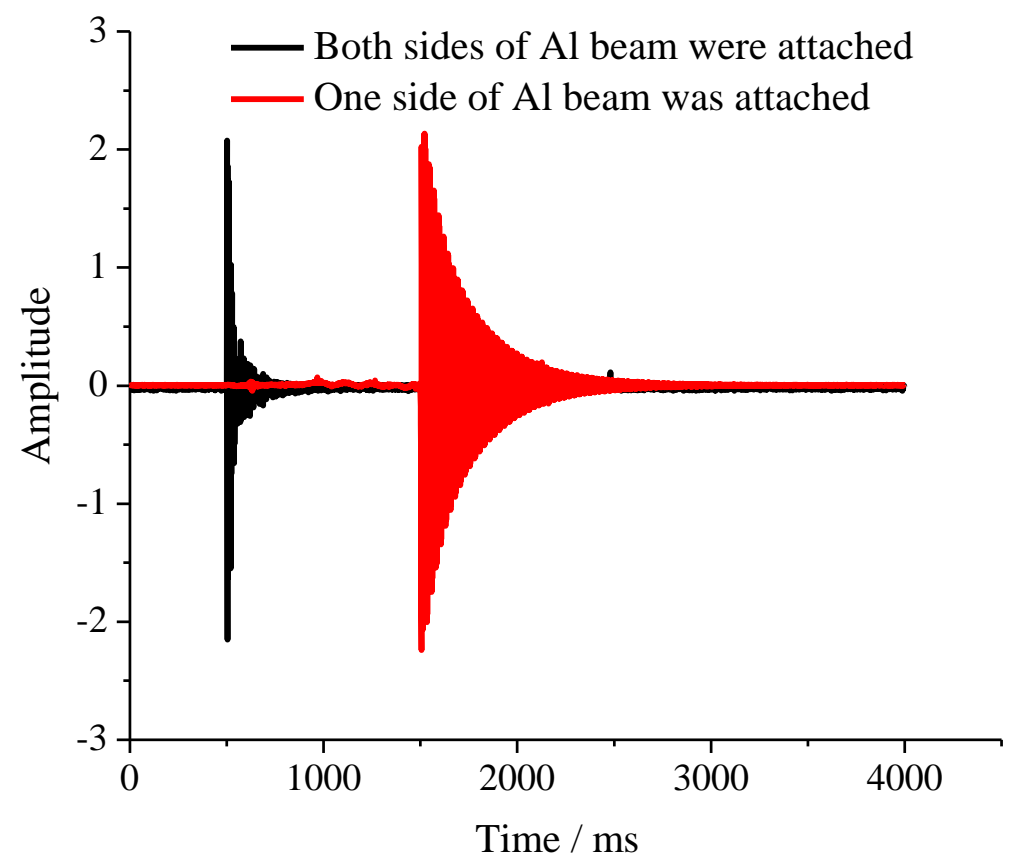

Figure 4.12 Comparison of free vibration damping test between PANI/11\% MWCNT single side covered (red) and PANI/11\% MWCNT double sides covered (black) Al base beam.

By comparing the vibration traces shown in the Figure 4.12, we noted that when the system vibrated from the same relative starting conditions, the double-sided coverage (black trace) attenuated the vibrations in a much shorter period of time than its single sided 
counterpart. In other words, the damping effect was dramatically increased when both sides of the $\mathrm{Al}$ base beam were covered by PANI/11\% MWCNT. This result follows the trends found in Chen and Levy (Chen and Levy, 1996) for a constrained double sandwich viscoelastic material covering an $\mathrm{Al}$ base beam.

Our PANI/MWCNT system was specifically created to replace such a constrained viscoelastic system, saving on weight of the constraining layer and the viscoelastic material, yet still maintaining the constraining layer stiffness through the MWCNT and introducing damping through the PANI for vibration attenuation.

\subsubsection{The Thickness Effect of Alu22minum Base Beam}

For the third test (see Figure 4.5 for the configuration of the test setup), the width of the PANI/MWCNT sample was $0.8 \mathrm{~cm}$, while the width of the Al beam was $0.9 \mathrm{~cm}$. Thus, $88.89 \% \mathrm{Al}$ beam was covered by the sample in the width direction. From the data shown in Figure 4.13, it could be seen that for $2.4 \mathrm{~mm}$ thickness Al beam, the highest damping ratio was 0.00492 when the beam was clamped at (a) in Figure 4.13. The damping ratio increased when the distance between the clamp and the sample was increased. It reached a maximum value of 0.00685 when the distance between the clamps to the sample was 5.4 $\mathrm{cm}$. Then, it decreased to 0.00452 when the distance was $7.7 \mathrm{~cm}$ from the clamp to the sample.

Meanwhile, for $1.25 \mathrm{~mm}$ thickness Al beam, the same pattern was deduced as described for the $2.4 \mathrm{~mm}$ thick beam but the values were higher. The damping ratio reached a maximum of 0.0079 when the distance from the clamp to the sample was $1.7 \mathrm{~cm}$. Also, compared with the sample attached onto the thick Al beam, the PANI/MWCNT attached onto the thin $\mathrm{Al}$ beam peaked, i.e., had higher damping ratios when the clamped condition 
occurred closer to the sample $(0.0082$ at approximately $2.5 \mathrm{~cm}$ from the sample vs. 0.0072 at approximately $4 \mathrm{~cm}$ from the sample), as shown in Figure 4.13 . The damping behavior shown based upon the beam thickness is also consistent with what was found by Chen and Levy (Levy and Chen, 1994; Chen and Levy, 1996).

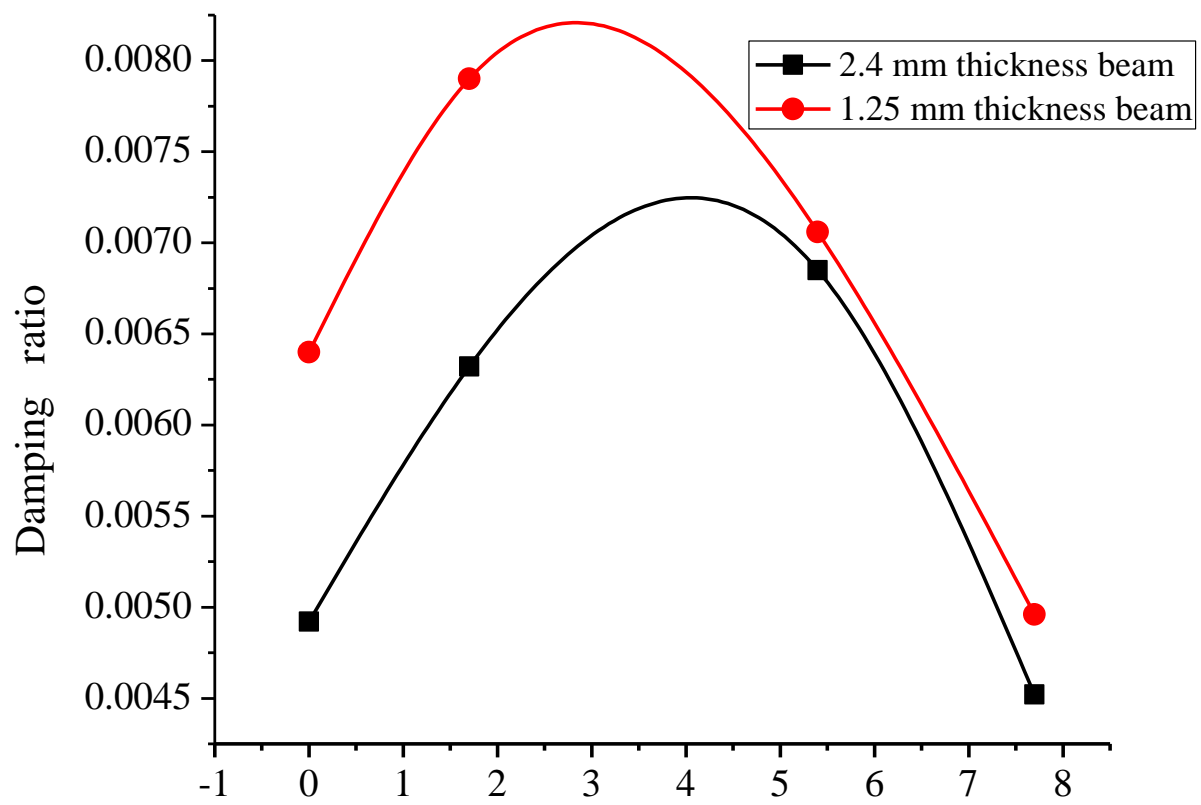

Distance from the clamp to the sample $(\mathrm{cm})$

Figure 4.13 Damping ratios of $2.4 \mathrm{~mm}$ and $1.25 \mathrm{~mm}$ thickness Al beams with different distance from the clamped side of the beam to the right end of the sample.

\subsubsection{Damping Ratios of Different Compression Force of PANI/MWCNT}

For the fourth test, the impact of the $289 \mathrm{~N}$ and $334 \mathrm{~N}$ "squeezing" force was studied (See Figure 4.6 for the configuration of test setup for the Al base beam with single side attachment). The free vibration trial results are shown in Figure 4.14.

Five trials were run for each of clamping locations listed. Standard deviation is also provided in Figure 4.14. The trend of damping ratios increased as the sample covered more 
of the beam. The effect of both the $289 \pm 3 \% \mathrm{~N}$ and $334 \pm 3 \% \mathrm{~N}$ applied squeezing force on the damping ratios were not a critical factor as is shown in Figure 4.14. For every single clamping location, the damping ratios for both $289 \pm 3 \% \mathrm{~N}$ and $334 \pm 3 \% \mathrm{~N}$ PANI/MWCNT samples had overlapped standard deviation, an indication that the results were not statistically different from each other. Therefore, the compression force used to create the PANI/MWCNT nanocomposites has very little impact on their damping ratios.

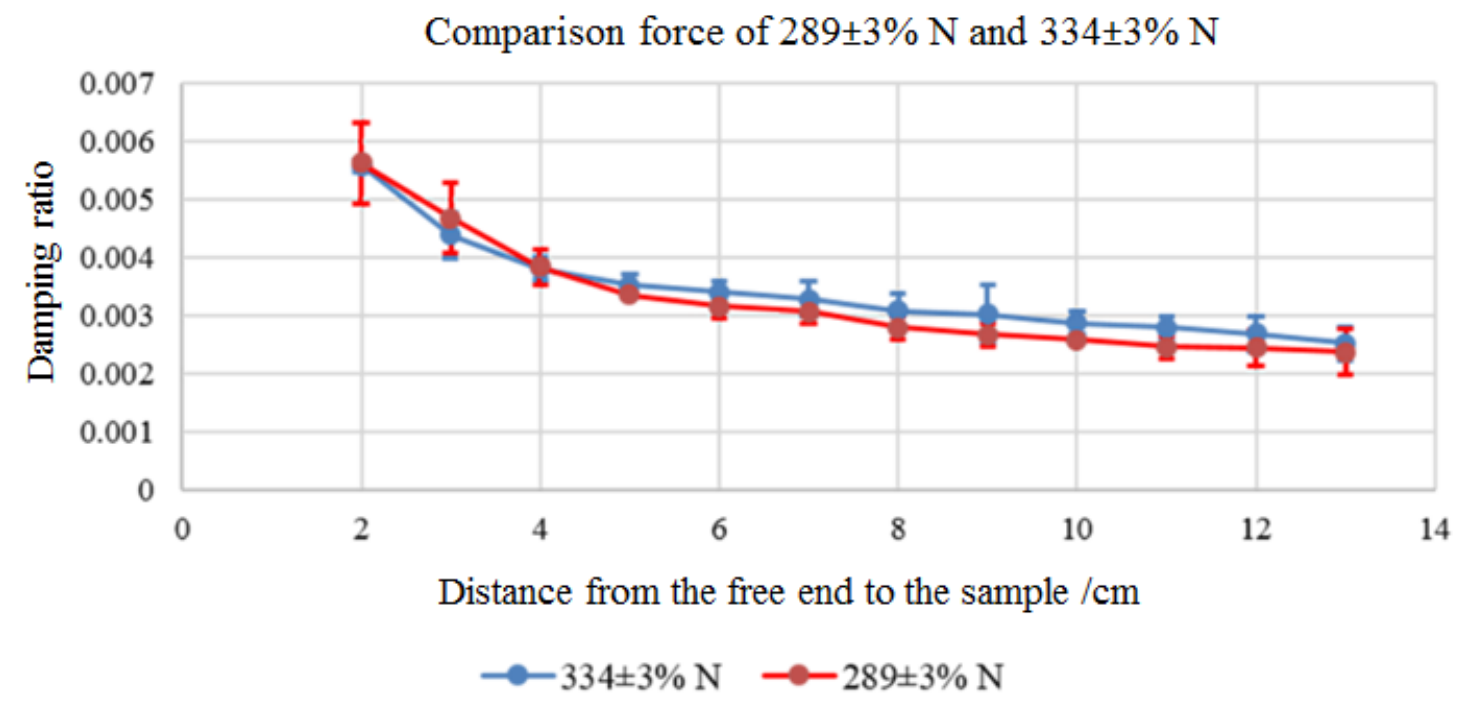

Figure 4.14 Damping ratio comparison between $289 \pm 3 \% \mathrm{~N}$ and $334 \pm 3 \% \mathrm{~N}$ compression force of PANI/MWCNT when clamped on the free end side. Error bars are provided for each test.

\subsubsection{Damping Results for Single Side Attachment}

As mentioned previously, the reason for the creation of the composite was for use as a sensor-actuator that also had damping capabilities. To check on the damping capability aspect, several tests were performed. Figure 4.6 is the configuration of the Al base beam single side attachment tests setup. Free vibration trial results are shown in Figure 4.15. 
Five trials were run for each of the clamped locations listed in Figure 4.6. The results clearly showed that the damping ratio increased as the distance from the free end to the sample decreased for clamping on both the free end side, ${ }^{*}$, and the sample side, \# (see Figure 4.15, red and green data respectively). However, when clamped on the sample side, \#, damping ratios were found to decrease as the distance from the free end to the sample was further decreased. After that, it increases again and achieved the highest damping value of 0.0131 .

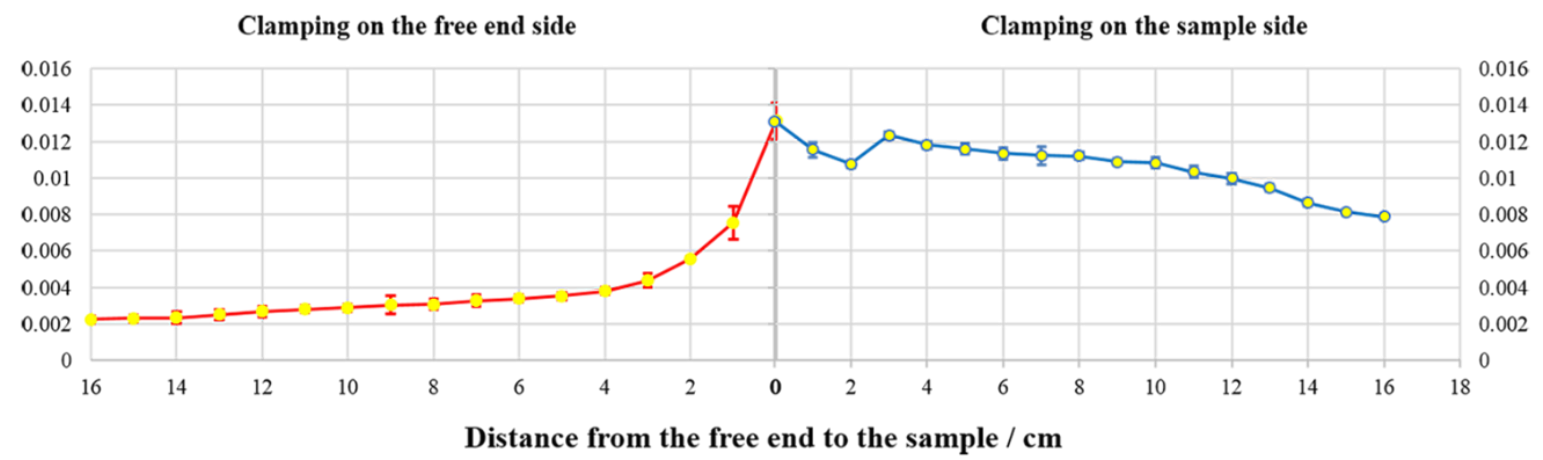

Figure 4.15 Damping ratios of single side attached PANI/MWCNT clamped on the sample side and on the free end side. Error bars are provided for each test.

In most cases, when the beam was clamped on the free end side *, the damping ratios were lower than when the beam was clamped on the sample side, \#. For example, the highest damping ratios was 0.0131 when clamped on free end side, *, where the distance from sample to the free end was $0 \mathrm{~cm}$. It was the only case for which both clamping conditions (free end side or sample side) were the same. However, when the distance from sample to the free end was $1 \mathrm{~cm}$ and clamping was on the free end side, *, the damping ratio was still lower (0.00757) than the damping ratio when clamped on the sample side, \#, 
and the free end was at its furthest location from the sample, i.e., $16 \mathrm{~cm}(0.00788)$. Finally, when the sample was symmetrically located with respect to the ends, the damping ratio values had to match.

\section{2.6.7 Damping Results for Single Side Attachment}

For the fifth test, namely the double sides' attachment setup shown in Figure 4.7, the damping ratio displayed similar type trends as the damping ratios for single sided attachment (See Figure 4.16). However, the double sides' attachment had overall higher damping ratios compared with single side attachment. It reached the highest damping ratio of 0.019 with double sides' attachment when the sample was $7 \mathrm{~cm}$ away from the free end. This meant that there was more uncovered beam when the maximum damping ratio was reached in this setup that in the single side attachment setup, and, that single side attachment required almost complete beam coverage to achieve the best damping ratio. Also, sample side clamping, \#, damping ratios were higher that free end side, ${ }^{*}$, clamping damping ratios.

The relationship of loss factor, $\eta$ and beam coverage $\mathrm{L} 1 / \mathrm{L}$ is presented for single sided attachment and double sided attachment for the two clamping conditions previously discussed. The results are given in Figure 4.17. The loss factor, $\eta$, is twice the damping ratio for small damping ratios up to 0.3 (Rao, 2004; Li and Levy, 2009). For sample side clamping, $\eta$ increased as coverage length $\mathrm{L} 1 / \mathrm{L}$ increased. Then the loss factor, $\eta$ decreased before increasing again as the coverage length $\mathrm{L} 1 / \mathrm{L}$ continued to increase. The relative coverage length $\mathrm{L} 1 / \mathrm{L}$ increased since the beam length was being decreased which the sample length L1 was unchanged. The trend for double sided attachment was found to follow the trends discussed by Rao (2004) and Levy and Chen (1994), i.e., higher lost 
factors occurred as sample length to beam length $(\mathrm{L} 1 / \mathrm{L})$ increased before decreasing as L1/L tended to 1 .

For beams clamped on the non-sample side, $*$, the loss factor, $\eta$, increased with coverage length, L1/L, though its value was several times smaller than its value when the beam was clamped on the sample side, \#. This is because the sample, in this case, acted as a small mass at the end of the beam which modified the coverage length effects (Chen and Levy, 1996).

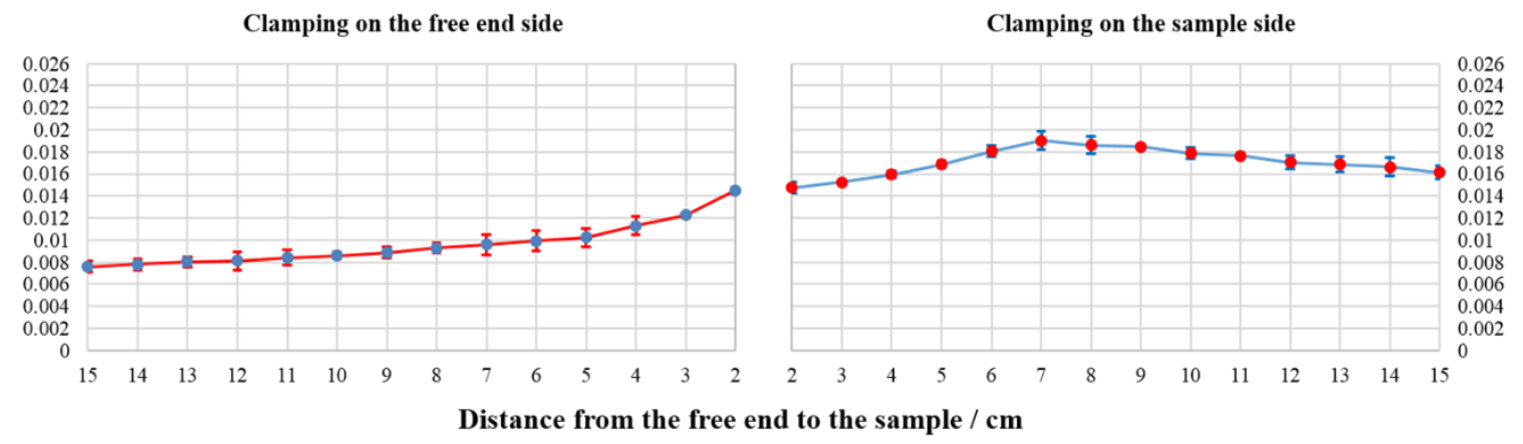

Figure 4.16 Damping ratios of both sides attached PANI/MWCNT clamped on the free end side and on the sample side. Error bars are provided for each test.
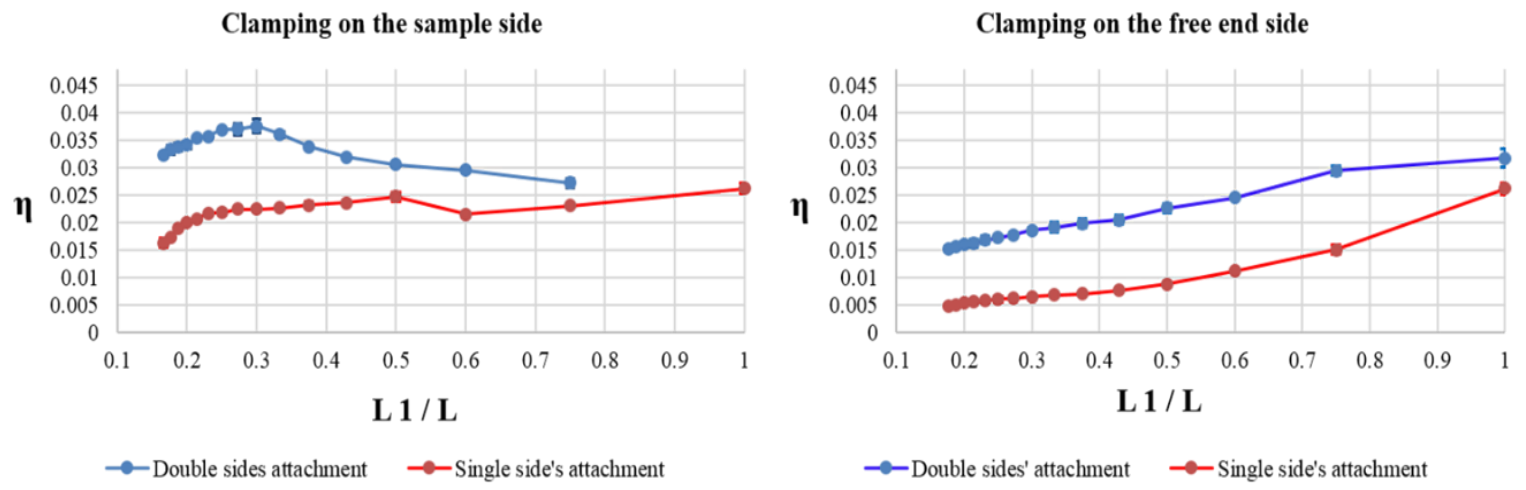

Figure 4.17 The relationship of loss factor $(\eta)$ and beam coverage $\mathrm{L} 1 / \mathrm{L}$ between single side and double sided attachment of the PANI/MWCNT films for both clamping conditions. 


\subsubsection{Strain Sensing Properties of PANI/MWCNT}

\subsubsection{Comparison of Different Components of PANI/MWCNT}

All three samples were covered with a thin layer of electrical non-conductive adhesive. The voltage output, $\mathrm{V}$, was found to vary too much during the first five minutes after the load was applied. The data recorded in Table 4.3 was after the loads had been applied for 5 min., and when the reading had stabilized already. During the first 5 minutes of the loading the data varied too much to record. From Figure 4.18, it showed that PANI/11\% MWCNT composite films data is linear, while the other two composites are not. It indicated that PANI/11\% MWCNT composite films have better linearity with regarding to the strain sensing properties than PANI $/ 22 \%$ MWCNT composite films and PANI $/ 44 \%$ MWCNT composite films.

Table 4.3 Raw data strain sensing test comparison of different components of PANI/MWCNT composite films.

\begin{tabular}{|c|c|c|c|}
\hline Loads/N & PANI/11\%CNT/mV & PANI/22\%CNT/mV & PANI $/ 44 \% \mathrm{CNT} / \mathrm{mV}$ \\
\hline 0 & -15.630 & -15.378 & -15.252 \\
\hline 2.4525 & -15.622 & -15.374 & -15.294 \\
\hline 4.4145 & -15.611 & -15.383 & -15.319 \\
\hline 6.3765 & -15.602 & -15.413 & -15.317 \\
\hline 8.3385 & -15.589 & -15.429 & -15.305 \\
\hline 10.3005 & -15.578 & -15.435 & -15.303 \\
\hline
\end{tabular}




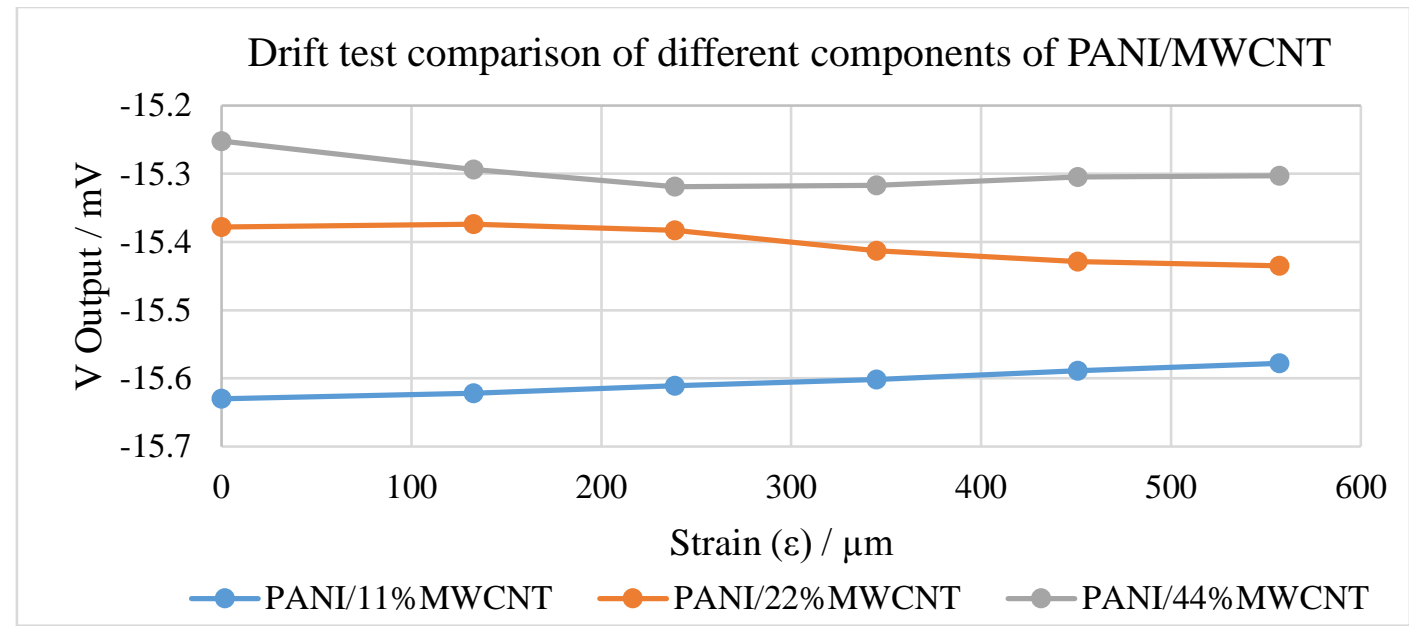

Figure 4.18 Strain sensor test comparison of different components of PANI/MWCNT.

\subsubsection{The Repeatability of Strain Sensing Property of PANI/ MWCNT Composites}

$1.962 \mathrm{~N}$ load were continued to be added onto one end of the cantilevered $\mathrm{Al}$ beam at 5 min interval. Then, it was unloaded all the loads and waited for $30 \mathrm{~min}$. Then, it was repeated the loading procedure twice more.

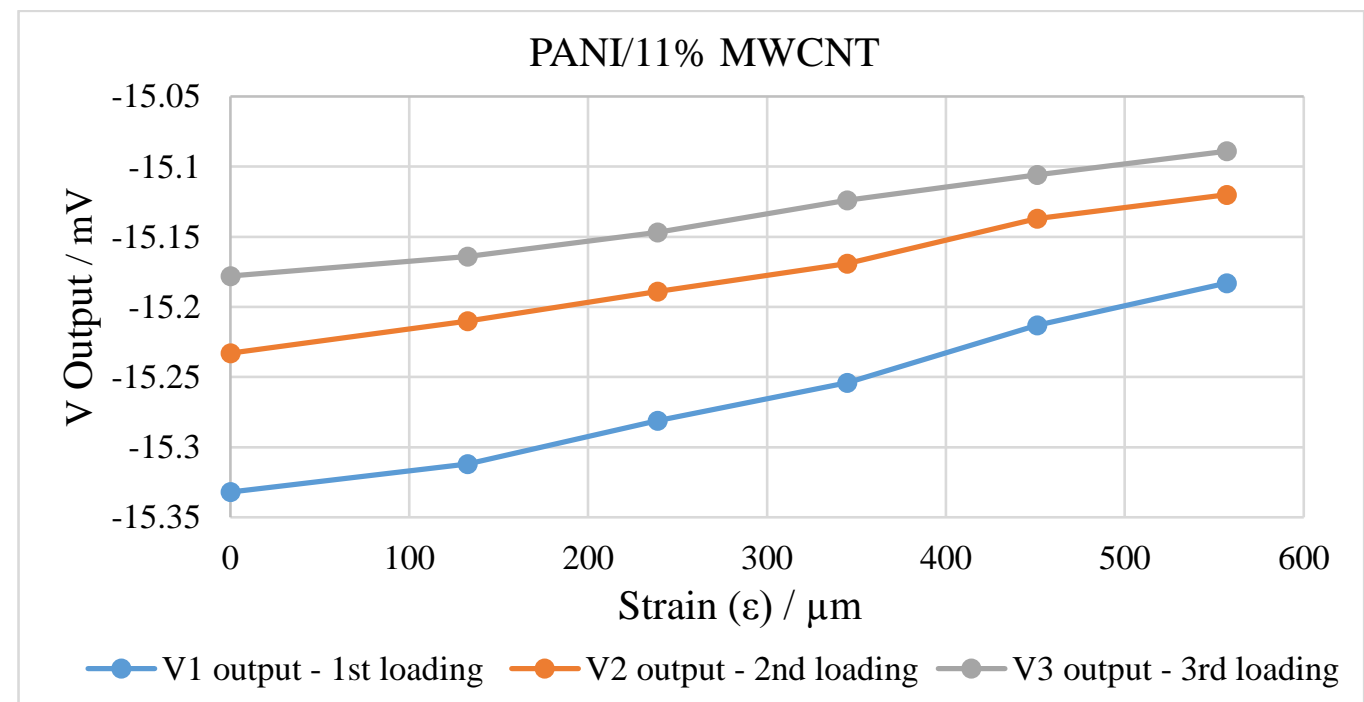

Figure 4.19 (a) The linearity and repeatability of strain sensor test of PANI/11\% MWCNT, PANI/22\% MWCNT and PANI/44\% MWCNT, showing load vs. output. 


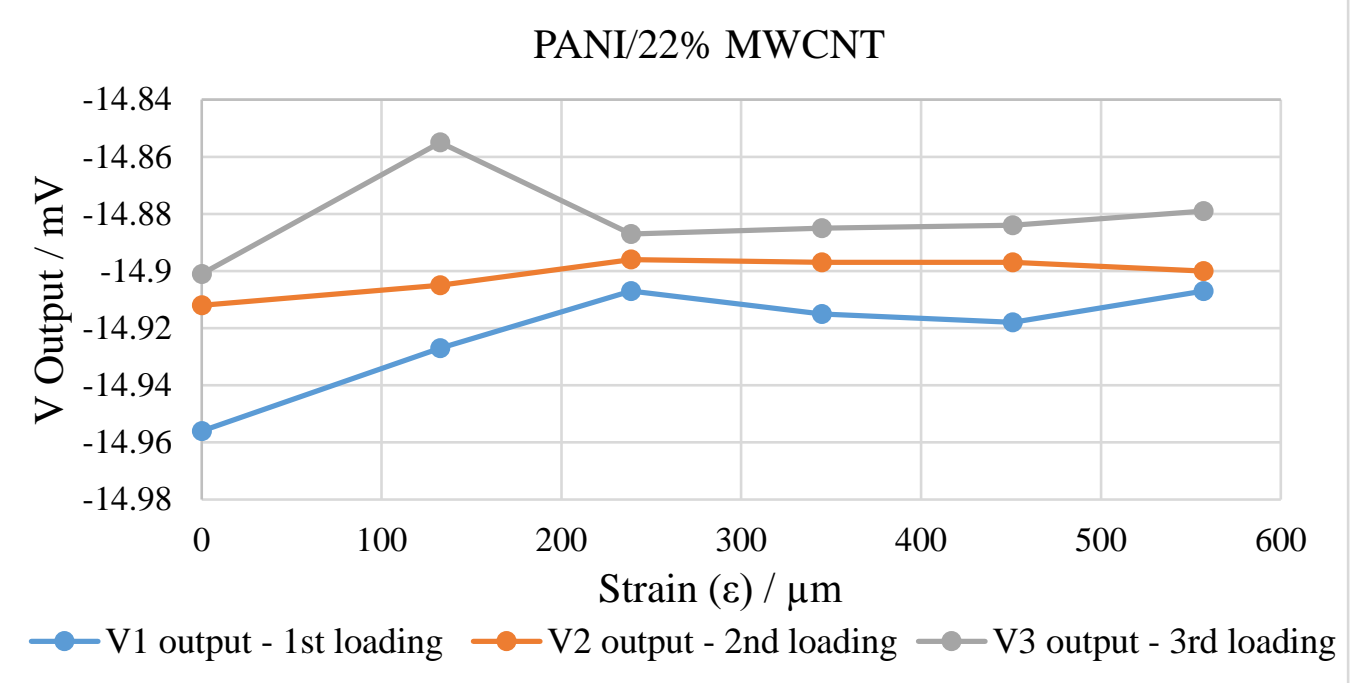

Figure 4.19 (b) The linearity and repeatability of strain sensor test of PANI/11\% MWCNT, PANI/22\% MWCNT and PANI/44\% MWCNT, showing load vs. output.

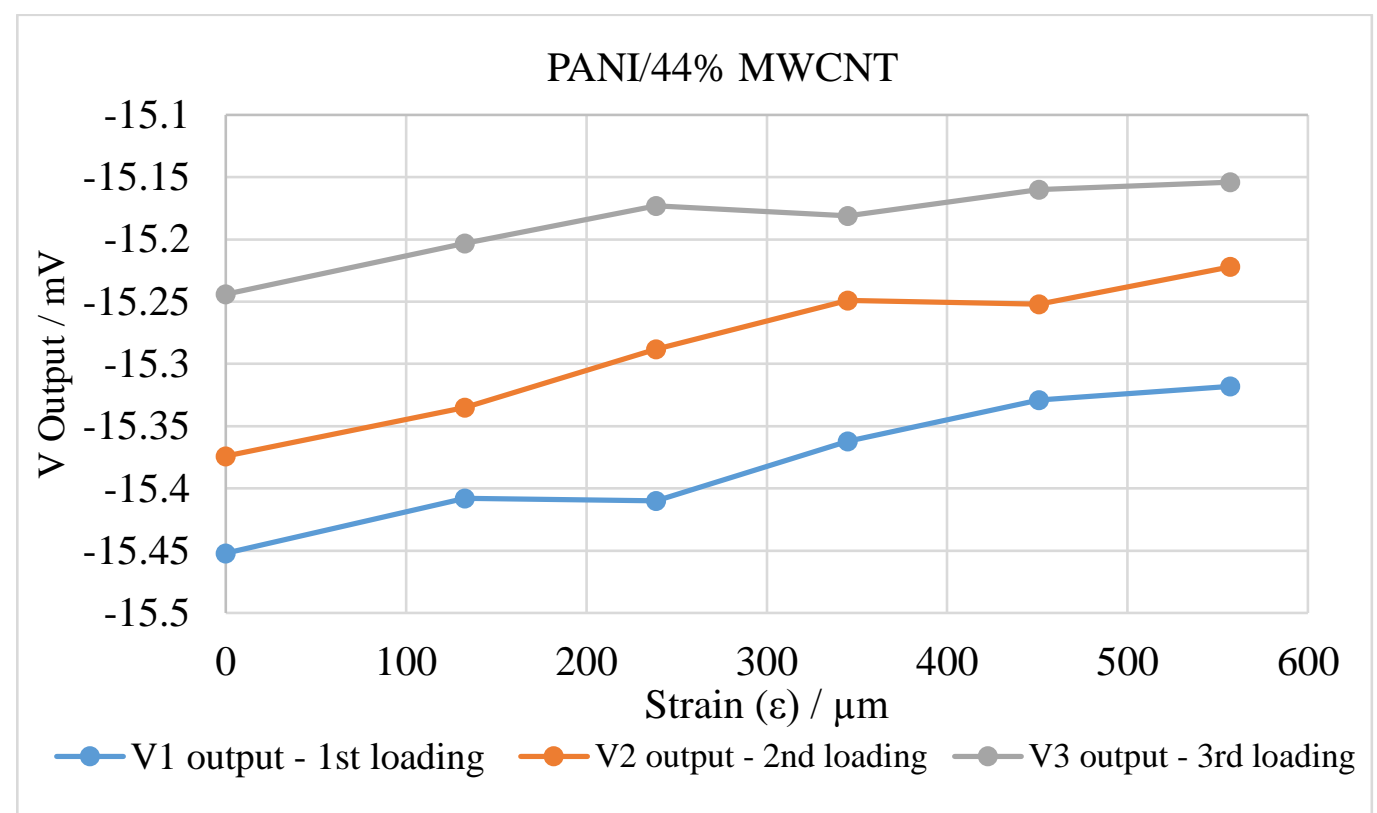

Figure 4.19 (c) The linearity and repeatability of strain sensor test of PANI/11\% MWCNT, PANI/22\% MWCNT and PANI/44\% MWCNT, showing load vs. output.

Though all the three composites varied a lot during each repeat process, the PANI/11\% MWCNT showed better linearity in each repeat process than the other two composites, 
namely PANI/22\% MWCNT and PANI/44\% MWCNT, which have shown in Figure 4.19 $(\mathrm{a}-\mathrm{c})$

\section{2.7.3 Dynamic Sensing Capability of the PANI/MWCNT}

Sensing capability of the sensor was evaluated by inputting a known frequency to the end of the beam with an attached sensor via a test bed to which the beam and sensor were attached (see Figure 4.20 for the test bed). The input signal applied was from 10 to 5000 Hz. Shown here in Figures 4.21 and 4.22 are results of the captured signals up to $1000 \mathrm{~Hz}$. Figure 4.23 represents the superposition of the previous graphs shown in Figures 4.21 and 4.22. This was undertaken to show that the PANI/MWCNT sensor was much more sensitive over a larger range of frequencies.
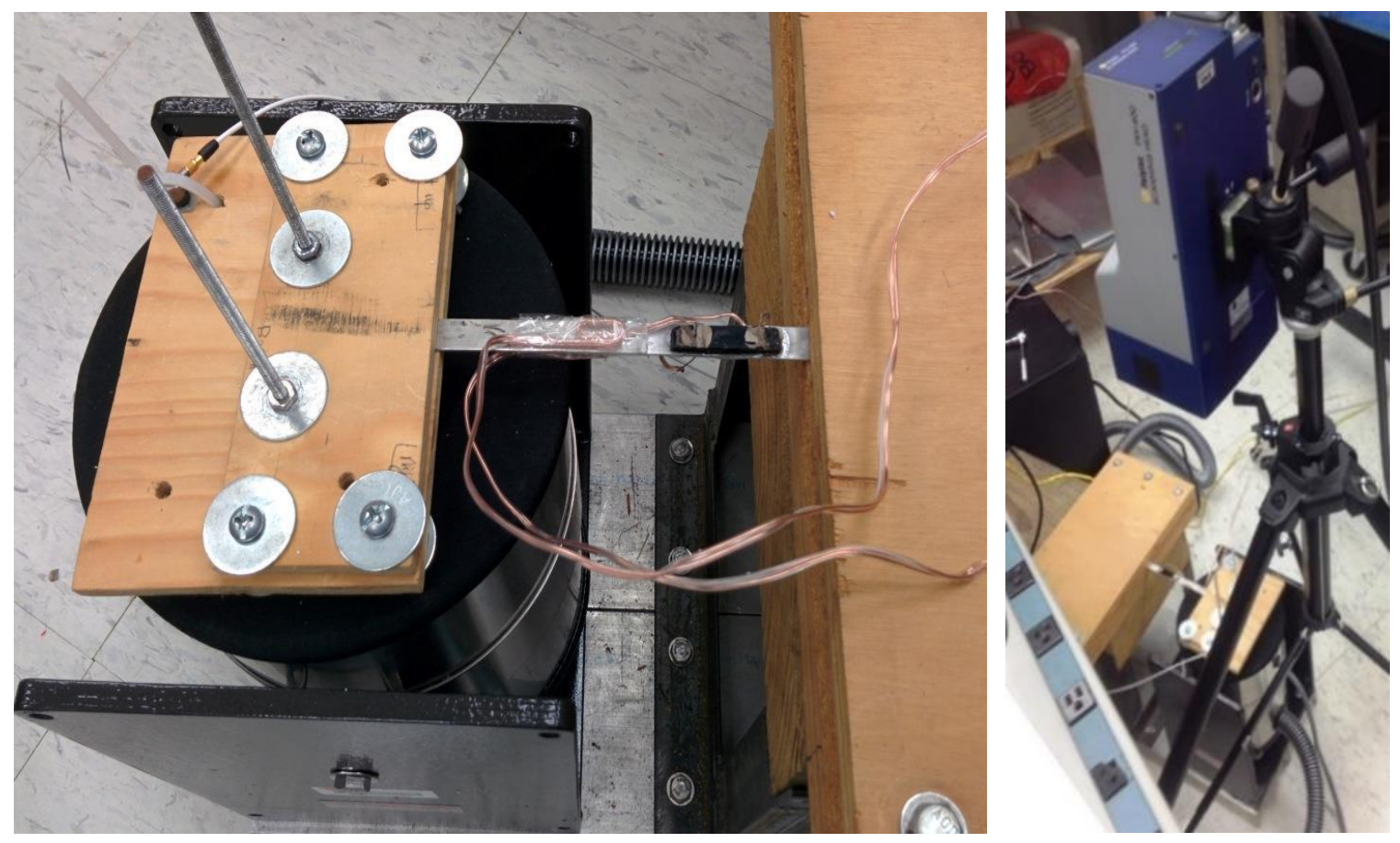

Figure 4.20 Beam and sensor in dynamic testing mode. Beam and sensor being interrogated by the laser vibrometer. 


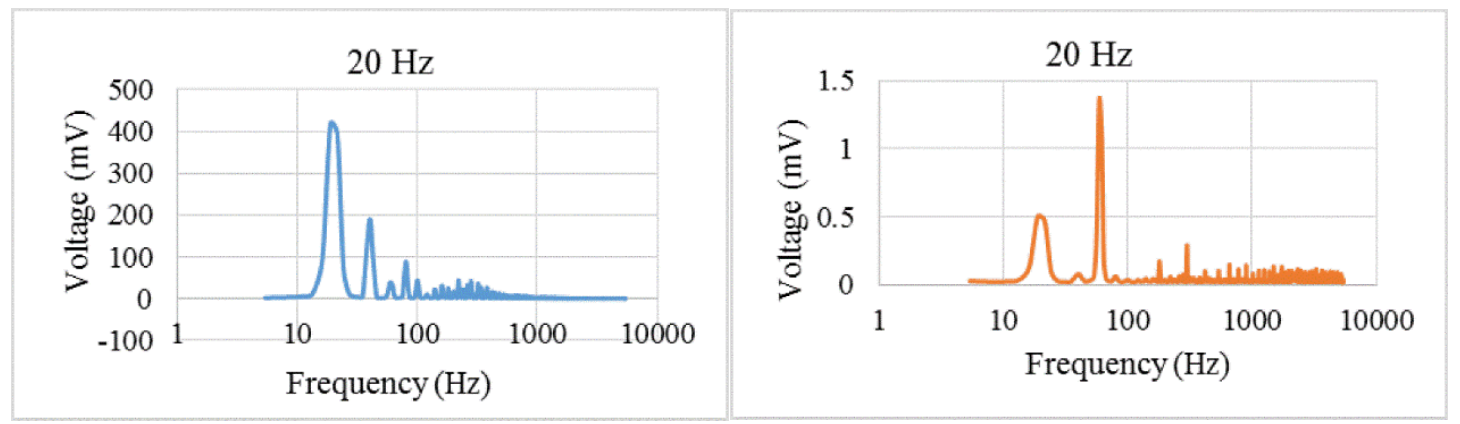

(a)PANI/MWCNT-Magnitude:418.030mV; (b)Foil Strain Gage-Magnitude:0.498 mV;
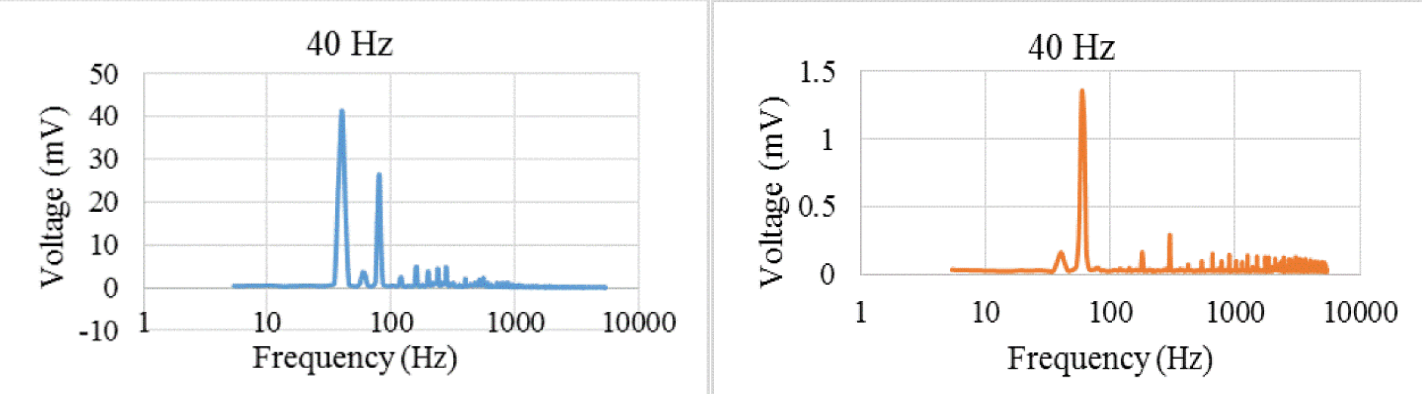

(c)PANI/MWCNT-Magnitude:41.296 mV; (d) Foil Strain Gage-Magnitude:0.163 mV;

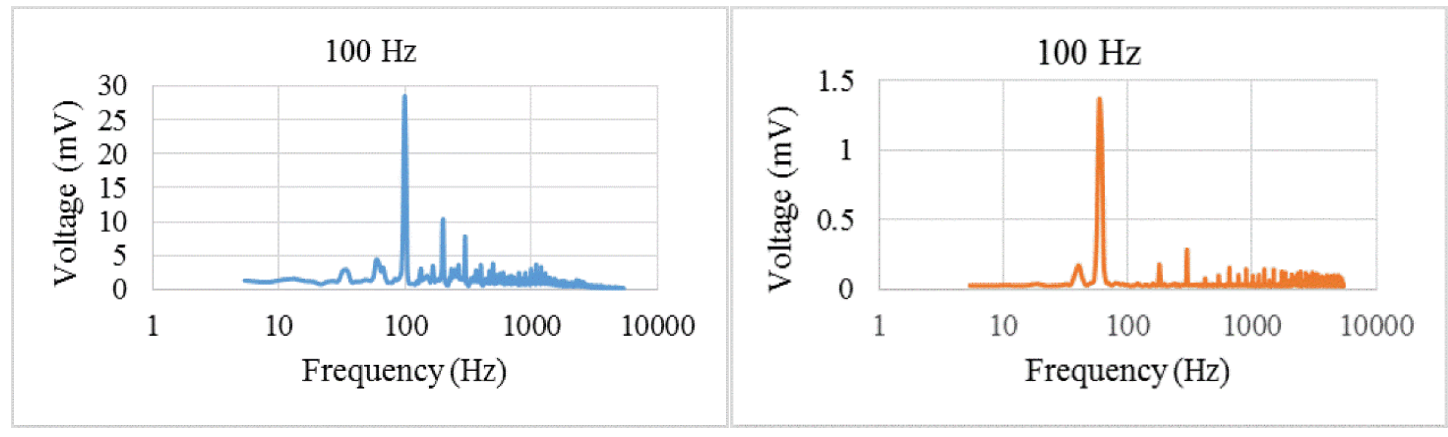

(e) PANI/MWCNT-Magnitude:28.278 mV; (f) Foil Strain Gage-Magnitude:0.031 mV.
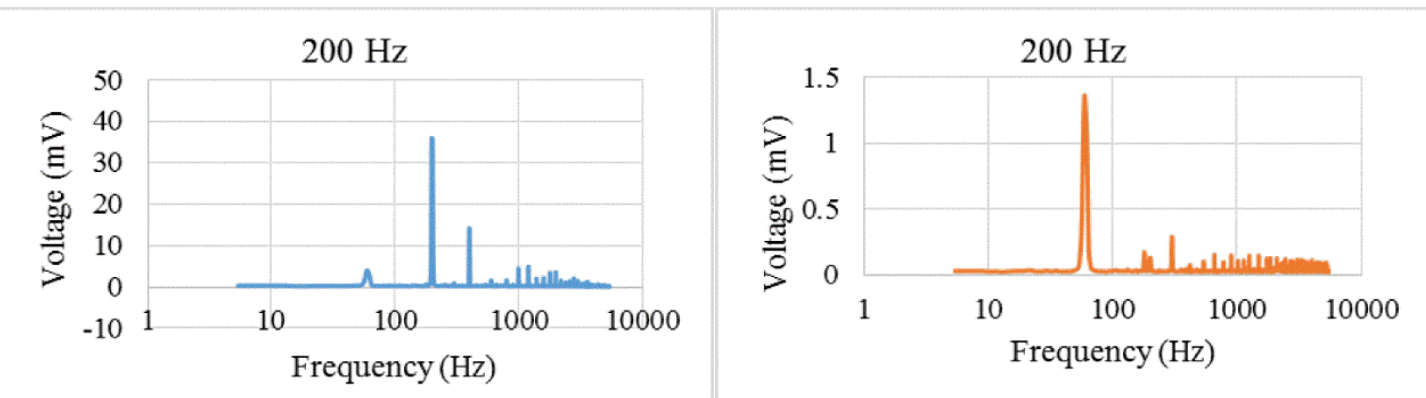

(g) PANI/MWCNT-Magnitude:35.981mV; (h) Foil Strain Gage-Magnitude: 0.130 mV;

Figure 4.21 Frequency response functions (FRF) of the PANI/MWCNT sensor and foil strain gage for frequency range $10-200 \mathrm{~Hz}$. 


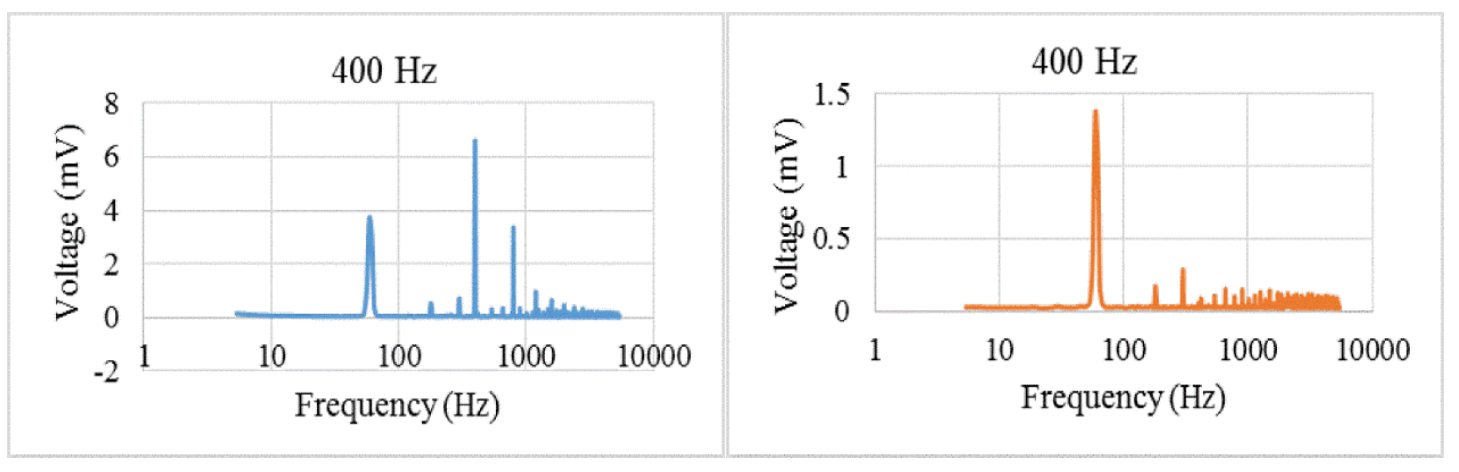

(a) PANI/MWCNT-Magnitude: $6.615 \mathrm{mV}$; (b) Foil Strain Gage-Magnitude: $0.058 \mathrm{mV}$;
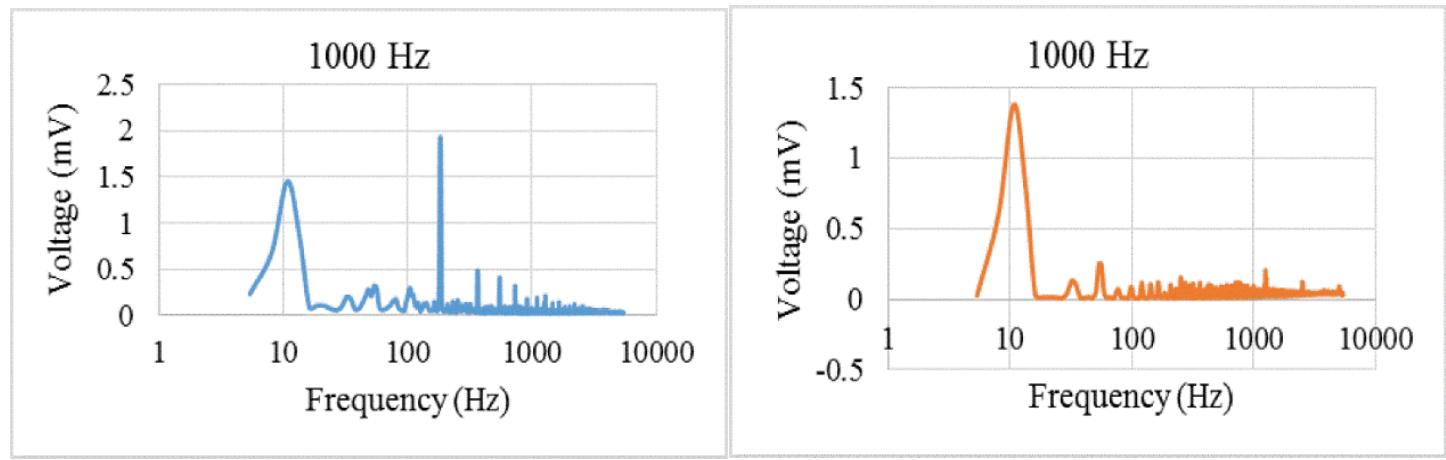

(c) PANI/MWCNT-Magnitude: $0.060 \mathrm{mV}$; (d) Foil Strain Gage-Magnitude: $0.064 \mathrm{mV}$.

Figure 4.22 Frequency response functions (FRF) of the PANI/MWCNT sensor and foil strain gage for 400-1000 Hz.

For Figures 4.23 (a-b) we noted that the magnitudes of the sensed signals were 100 times higher for the sensor than for the strain gage, whereas in Figures 4.23 (c-d) the magnitudes for the sensor were on the order of 20 times higher than those sensed by the strain gage. It was found that the PANI/MWCNT sensor was much more sensitive than the foil strain gages up until $400 \mathrm{~Hz}$ and the signal produced the highest peak in the FRF graphs. Beyond that frequency, either the two produced similar magnitudes or were not the highest peaks distinguishable. The magnitudes listed represent the signal height sensed by the PANI/MWCNT sensor (blue) and the strain gage (orange). 

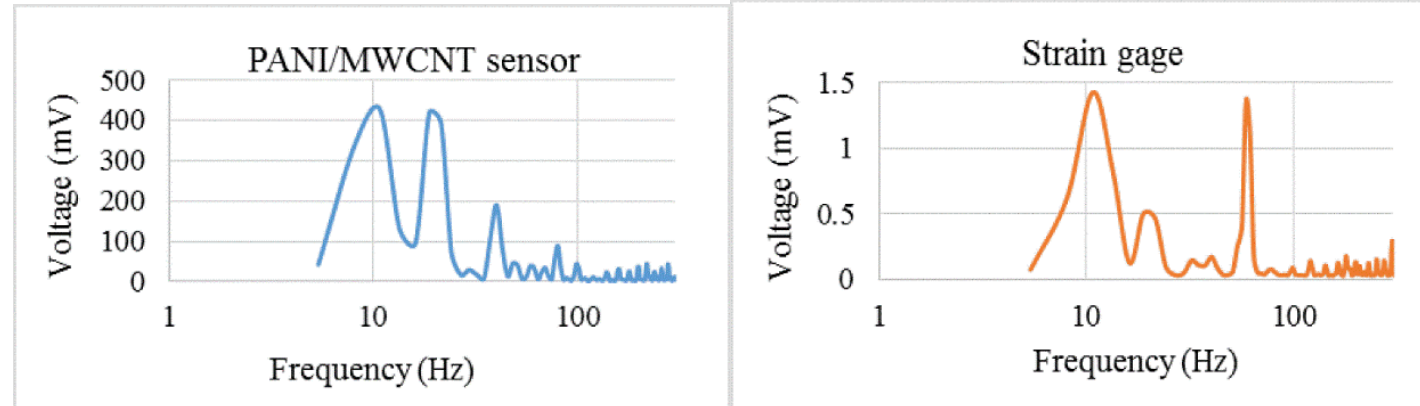

(a) FRF-PANI/MWCNT-sensor (10-1000 Hz); (b) FRF-Foil strain gage (10-1000 Hz);
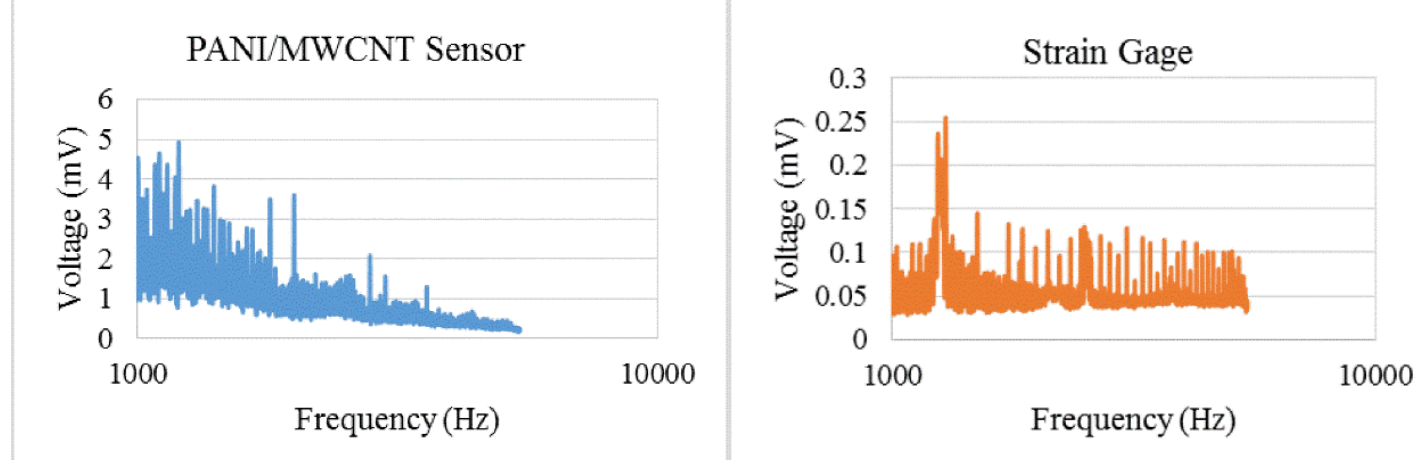

(c) FRF - PANI/MWCNT (1000 - 5000 Hz); (d) FRF- oil strain gage $(1000$ - $5000 \mathrm{~Hz})$.

Figure 4.23 Superposition of the frequency response functions of the PANI/MWCNT sensor and foil strain gage for low and high frequency. Graphs represent a superposition of the previous graphs to indicate the superior sensitivity of the PANI/MWCNT in the $10-1000 \mathrm{~Hz}$ range and the $1000-5000 \mathrm{~Hz}$ range.

\section{2.7.4 The Drift Test of PANI/MWCNT Sensor}

To determine the stability of the sensor, a drift test was employed. The drift test was carried out for the sample and for the strain gage for a period of 30 minutes or more (see Figures 4.26-4.27). The voltage data were captured and for the sensors were shifted so that average recorded signal value was zero. This allowed for direct comparison of the sensors on the graph. The change in voltage per hour for typical results were also tabulated below (see Table 4.4). The correlation of the data was small because significant noise was generated in the results due to the high sampling rate (see, e.g., Figure 4.27). Therefore, other measurements given in succeeding sections do not 
include the correlation values. Figure 4.26 shows typical drift test results for one strain gage and three sample sensors. Sensor 3 data is given as the orange trace in the figure and its trend line is given also by the orange dotted line in the figure. It was noted that the strain gage (blue trace) showed almost no changes over the test period though there was a slight downward slope to the line. The sample sensors exhibited very small slopes, as well. Table 4.4 provides the voltage changes for the same sensors and strain gage of Figure 4.26. Figures 4.27 (a-b) show typical sampling data for one of the samples and the strain gage where the sensor sample's voltage changes by $-0.1298 \mathrm{mV} / \mathrm{hr}$ while the foil strain gage changes by $-0.0866 \mathrm{mV} / \mathrm{hr}$ (the black dotted lines in the figures). The results of this testing showed that some sensor samples were unstable and had significant drift while others were stable but consistently had a drift that was of the same order of magnitude as the strain gage. This result was reasonable since our testing apparatus could not be moved and there was no way to isolate our samples from outside vibrations. Foil strain gages are known to be stable in this type of environment.

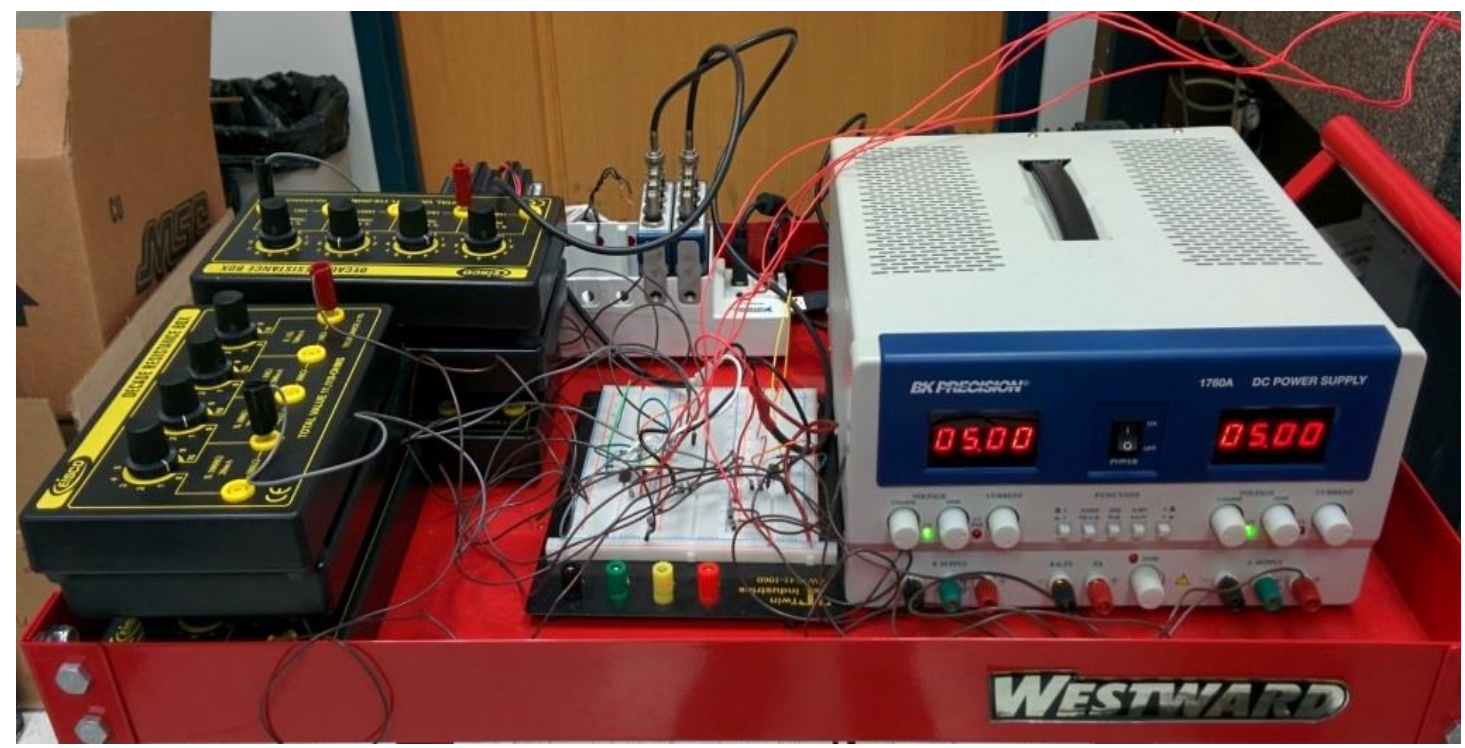

Figure 4.24 Wheatstone bridge setup for load sensing. 


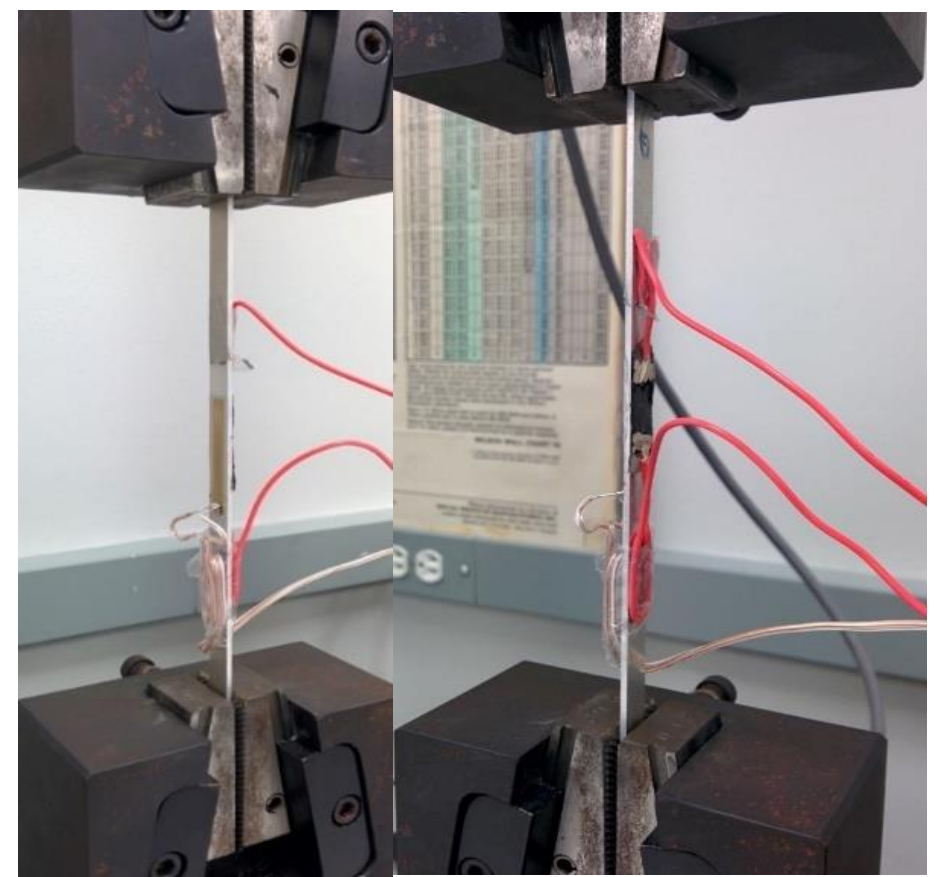

Figure 4.25 Same beam with strain gage showing and with sensor showing, both in loading mode.

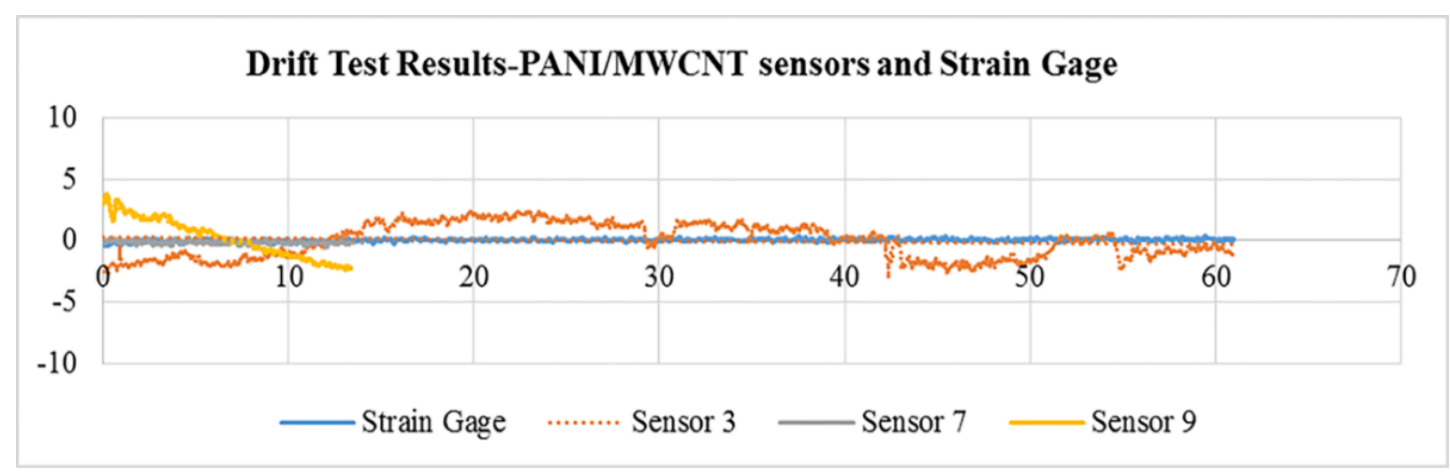

Figure 4.26 PANI/MWCNT sensor and strain gage drift test. Drift trend lines for typical PANI/MWCNT sensor and strain gage. Vertical scale is in $\mathrm{mV}$; horizontal scale is in minutes.

Table 4.4 Trend line Slopes for Figure 4.26

\begin{tabular}{|l|l|l|l|}
\hline Sample & $\Delta \mathrm{mV} /$ hour & Sample & $\Delta \mathrm{mV} /$ hour \\
\hline Sensor 3 (orange trace) & -0.1298 & Sensor 9 (gold trace) & -24.008 \\
\hline Sensor 7 (gray trace) & -0.4353 & Strain Gage (blue trace) & 0.1773 \\
\hline
\end{tabular}




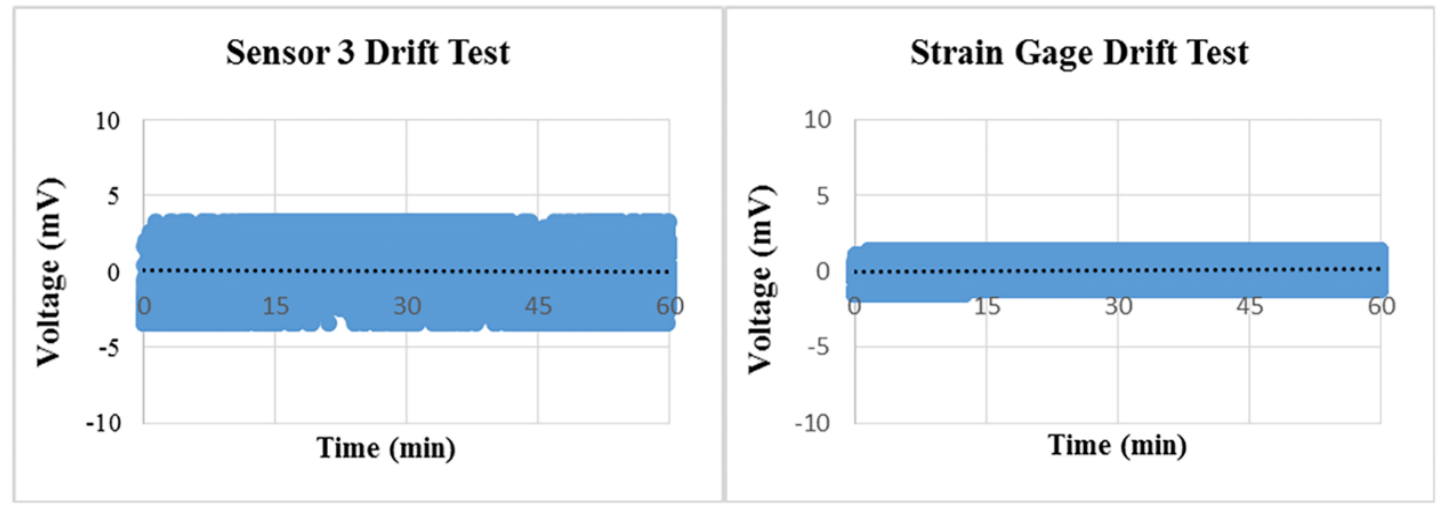

Figure 4.27 Drift test for PANI/MWCNT (sensor 3) and drift test for strain gage.

\subsubsection{Linearity and Repeatability of PANI/MWCNT Sensor}

Linearity and repeatability are two very important characteristics for strain sensors.

It was desirable that the relationship between the applied strain and the output voltage be linear. The source voltage for the sensor and strain gage in Figure 4.28 were equivalent.

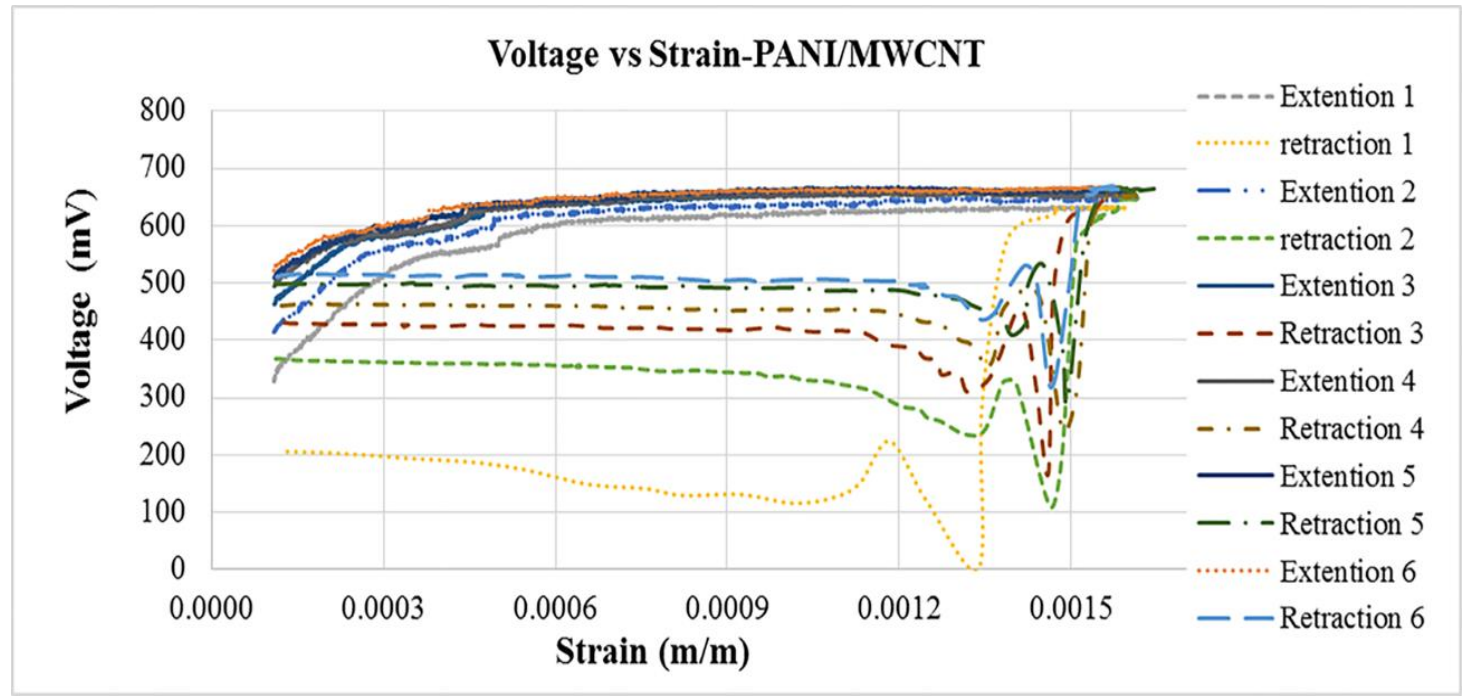

Figure 4.28 Voltage-strain curves for the PANI/MWCNT sensor. Note initial linearity of the sensor then marked change in slope in which there is an apparent step, then linear once again at much lower slope. 
Table 4.5 Slopes and intercepts for the PANI/MWCNT sensor given in Figure 4.28.

\begin{tabular}{|l|l|l|l|l|l|l|}
\hline Slope [mV/strain] & Run 1 & Run 2 & Run 3 & Run 4 & Run 5 & Run 6 \\
\hline Section 1 & 810574 & 832298 & 726813 & 606265 & 554590 & 508097 \\
\hline Section 2 & 270135 & 212610 & 216208 & 184250 & 210864 & 190580 \\
\hline Section 3 & 25577 & 26860 & 26117 & 18852 & 20537 & 26283 \\
\hline Intercept [mV] & Run 1 & Run 2 & Run 3 & Run 4 & Run 5 & Run 6 \\
\hline Section 1 & 265.91 & 329.22 & 393.02 & 438.98 & 455.68 & 476.29 \\
\hline Section 2 & 440.67 & 492.51 & 514.36 & 528.52 & 531.09 & 541.62 \\
\hline Section 3 & 594.96 & 607.21 & 624.92 & 630.15 & 635.24 & 628.53 \\
\hline
\end{tabular}

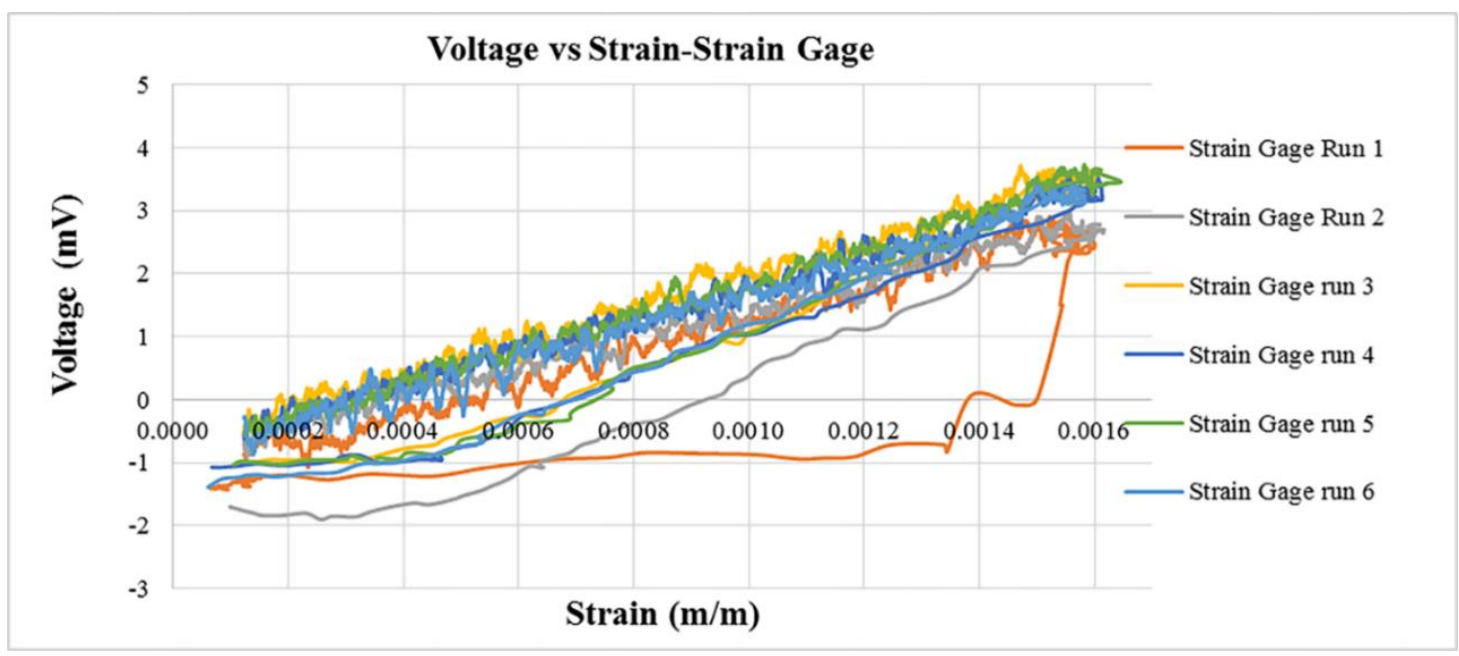

Figure 4.29 Voltage- strain curve for strain gage. The strain gage shows linear Voltagestrain behavior until unload and returns to the same start value.

Figures 4.28 and 4.29 show typical data for samples that responded as desired.

This dataset of one such sample shows six quasi-static loadings and instantaneous unload cycles. This figure also provides typical data obtained from testing both the 
sample sensor and the strain gauge. Shown are the results of the strain sensing capability of PANI/11\% MWCNT composites sensor and this information is compared to the standard foil strain gage. Each test consisted of a 4-5 minutes' pre-load preparation period, a three-minute loading period and a 3 second unloading period. Generally, both the sensor's and the strain gage's electric resistance change was essentially linear to the applied strain and was related to the gage factor. Whereas the strain gage's gage factor applied over the entire range, for the PANI/11\% MWCNT, this had to be recalculated over three ranges. The first 4-5 minutes of the test (not shown here) indicated that the system was in pre-load mode and had a residual strain to which both the sensor and strain gage had to adapt before the test was begun. Each unloading occurred about 3 seconds after releasing the previous load. Again, a "no load" pre-load period was introduced before the loading was begun for the next test. The strain gage appeared to have returned to "zero" but the PANI/MWCNT sensor appeared to show some hysteresis. The standard foil strain sensor responded quickly according to the load applied to the beam. However, PANI/MWCNT composites sensor did not completely return to "zero".

As a new load was applied increasing the strain, the PANI/MWCNT composite sensor responded and sensed the strain initially linearly with the input. Yet, the slope for the sensor appeared to have 3 distinct regions: rapid change up to strains of 0.0002 0.0004 , then a lower slope to about a strain of $0.0004-0.0006$, then an even lower slope over the remainder of the test cycle before the load was removed. However, when one evaluated the six cycles we noted the similarity of the load-time graphs. We noted that 
the sensor was more sensitive showing changes in voltage that were several orders larger than those of the strain gage. This was true for all 3 regions. For the strain gage, the results of 6 tests were similar. Therefore, it was concluded that the PANI/MWCNT composite sensor sensed similarly to the conventional foil strain gage but the slope of the load displacement curve (i.e., voltage/strain curve) was not uni-valued, though in terms of repeatability and linearity it was repeatable. When one compared the slope of the stress-strain curve in these three regions (not shown here), the slopes were found to be within a few percent of each other.

We also noted a small step in the load time curve towards the middle of the second linear region, which indicated that the internal makeup of the PANI/MWCNT underwent some type of change, perhaps slippage of the MWCNT over each other, thereby reducing the sensor gage factor. We also noted that when the load was suddenly released a "wave-like" behavior appeared in the trace and was due to the tensile machine not having an unloading program that used an unloading rate that was the negative of the loading rate. Yet, as the sensor cycled through several cycles the unloading trace and the loading trace tended toward stable and similar traces.

\subsection{Conclusions}

Different composites of PANI/MWCNT sensors were created to evaluate their morphological properties and sensing and damping capabilities versus a standard foil strain gage. The sensor's damping capabilities was also compared with a constrained layer damping system. 
PANI/MWCNT nanocomposites were synthesized through an in situ polymerization method. Frit compression method was introduced to make PANI/MWCNT nanocomposite films/discs. SEM and TEM results showed that this technique produced a coating of PANI onto the MWCNT.

Preliminary free vibration tests result showed that PANI/11\% MWCNT displayed higher damping ratio than pure PANI, PANI/22\% MWCNT, PANI/44\% MWCNT and pure MWCNT, meaning that further addition of MWCNT to increase the electrical conductivity of the composite reduced the damping capability of the PANI/MWCNT composite. The location of the clamped support of the cantilever beam was very important to the magnitude of the damping ratio while performing the free vibration damping tests. When the beam was clamped close to the sample end, with the sample between the clamp and the free end, it displayed higher damping ratios than when the beam was clamped to the free, uncovered end.

Damping test were also run for both single and double sided PANI/11\% MWCNT coverage on $\mathrm{Al}$ base beam. The results showed that by attaching the nanocomposite sample to both sides of $\mathrm{Al}$ beam, the damping ratio was much higher than for the single sided coverage. For example, the damping ratio reached a value of 0.072 when both sides were attached, which was 18.85 times higher than the single side attached with clamping on the sample side. Also, the free end vibration test results showed that for the single sided and double sided attachment damping ratios followed the same trends. However, the double-sided attachment was found to have higher damping ratios when the sensor was placed close to the clamped end of the beam. 
The relationship of loss factor, $\eta$, and beam coverage length, L1/L, was discussed for single sided attachment and double sided attachment. The loss factor, $\eta$, increased as coverage length, $\mathrm{L} 1 / \mathrm{L}$, increased. The loss factor, $\eta$, then decreased before increasing again as the coverage length, L1/L, continued to increase. The trend for double sided attachment was found to follow the trends discussed by Rao (2004) and Levy and Chen (1994).

The thinner $\mathrm{Al}$ base beams were found to have higher damping ratios than the thicker Al base beams. The damping ratios were again found to have a maximum that depended on the amount of coverage of the beam by the sample. This could be explained by the fact that the beam's critical damping constant, which equals $(4 \mathrm{~km})^{1 / 2}$, depended on the square of the beam's height if one used a single degree of freedom representation of the beam-sample system. Since the damping ratio was inversely proportional to the critical damping constant, then decreases in beam height caused increases in damping ratio, as experimentally shown.

The PANI/MWCNT sensor's relation between load and displacement or strain cannot be described by a single equation as found for a strain gage. The loaddisplacement relationship appeared to have three regions in which the relationship was quasi-linear. This was in line with an observation reported elsewhere (Levy, 2014), namely, adding too much PANI to the sensor patch tended to destroy the linear sensing capability of the sensor patch.

Loading cycle tests showed that though there appeared to be residual strain in PANI/MWCNT sensor after the load was removed, the PANI/MWCNT sensor seemed 
to lag and showed some hysteresis in the next loading cycle. However, both the sensor and the foil strain gage reacted linearly when re-engaged.

The dynamic sensing test results showed that over the range of $10-1000 \mathrm{~Hz}$, the PANI/MWCNT composite sensor was consistently superior to the foil strain gage for sensing purposes since the highest peak consistently corresponded to the input frequency and was much higher (e.g., 820 times higher at $20 \mathrm{~Hz}$ ) than those of the strain gage. At higher frequencies, the composite sensor performed better but the signal peaks were not the largest.

Drift test of the sensor showed that it was stable. Other drift tests performed (Levy, 2014), showed that the PANI/MWCNT sensor was stable over many hours changing by $0.25 \mathrm{mV}$ only over a $20 \mathrm{~h}$ period.

Lastly, not all samples created were capable sensors. However, the ones that worked produced repeatable results. In comparison with viscoelastic (VEM) constrained layer dampers, the same size PANI/MWCNT sensor may or may not be a weight saver; but would surely be cheaper to produce. 


\section{CHAPTER V}

PREPARATION OF POLYURETHANE/MULTIWALLED CARBON NANOTUBE

AND CHARACTERIZATION OF THEIR STRUCTURAL DAMPING AND

\section{STRAIN SENSING PROPERTIES}

\subsection{Introduction}

Theoretical analysis have shown that the addition of CNT into a polymer matrix can improve the damping ratio and stiffness of the matrix (Xu, 2013; Zhou et al., 2004), while experiments of different MWCNT composite films have verified the theoretical prediction (Xiong et al., 2006; Koratkar et al., 2002). Furthermore, MWCNT films have been demonstrated to show the capability of being strain sensors (Li et al., 2008; Zhou et al., 2004). PU has been shown to have unique dynamic properties in combination with its high load-bearing capacity, properties which were broadly studied for vibration control applications. Studies have been completed testing the damping properties of MWCNT in PU matrix, for example, Sasikumar et al. (2012) and Xiong et al. (2006). However, due to the large aspect ratio of the CNT, the dispersion and directional alignment of the MWCNT in the polymer matrix was still found to be a great challenge.

Herein, several methods were used to make PU/MWCNT/ZnO nanorods/ nanowires composites films and PU/Buckypaper films. The advantages and disadvantages of each method were compared. Finally, a layer by layer attachment method was employed to make PU/Buckypaper composites, and then they were evaluated for their sensing and damping capabilities versus a standard foil strain gage 
and constrained layer damping system. A free vibration test method was adopted to study their damping properties. Static and dynamic strain sensing properties were also investigated. One of the advantages of such a composite sensor was its light weight compared to a passively constrained layer damper.

\subsection{Experimental Sections}

\subsubsection{Mold Casting Method to Make Pu/MWCNT/ZnO Nanowires Composites}

Piezoelectric materials, as the most popular smart material in the last two decades, had been successfully used in many fields. When the aspect ratio of $\mathrm{ZnO}$ nanowires was larger than 100 , those $\mathrm{ZnO}$ nanowires were found to have piezoelectric properties (Zhao et al., 2004). When the piezoelectric $\mathrm{ZnO}$ nanowires and CNT were embedded into polymer matrix, it was expected that an internal circuit will be formed, which changes the electric energy into joule heating, thereby enabling this composite's vibration control properties.

\subsubsection{The procedure of Fabrication $\mathrm{ZnO}$ nanowires}

$\left(\mathrm{NH}_{4}\right)_{2} \mathrm{CO}_{3}$ and $\mathrm{Zn}\left(\mathrm{NO}_{3}\right)_{2} \cdot 6 \mathrm{H}_{2} \mathrm{O}$ were dissolved in distilled water to form $1.0 \mathrm{M}$ of $\left(\mathrm{NH}_{4}\right)_{2} \mathrm{CO}_{3}$ solution and $1.0 \mathrm{M}$ of $\mathrm{Zn}\left(\mathrm{NO}_{3}\right)_{2}$ solution, respectively. $10 \mathrm{ml}$ of $1.0 \mathrm{M}$ $\mathrm{Zn}\left(\mathrm{NO}_{3}\right)_{2}$ solution was dropped slowly into $20 \mathrm{ml}$ of $1.0 \mathrm{M}\left(\mathrm{NH}_{4}\right)_{2} \mathrm{CO}_{3}$ solution, then stirred vigorously to obtain a precipitate. The precipitate was filtered and repeatedly rinsed with distilled water to remove the residual reactants. Then, it was dispersed in $70 \mathrm{ml}$ distilled water by stirring for $5 \mathrm{~min}$. The obtained suspension was transferred into a Teflon-lined $100 \mathrm{ml}$ hydrothermal reactor. The hydrothermal synthesis was conducted 
at $160{ }^{\circ} \mathrm{C}, 180^{\circ} \mathrm{C}$ and $200{ }^{\circ} \mathrm{C}$, for $12 \mathrm{~h}, 16 \mathrm{~h}$ and $20 \mathrm{~h}$ in an oven (See Table 5.1), respectively. After the reaction was completed, the white product was collected and washed by alcohol. Finally, the $\mathrm{ZnO}$ nanorods/nanowires were obtained after drying at $90{ }^{\circ} \mathrm{C}$ for $12 \mathrm{~h}$ (Kim et al., 2011.).

The morphology of the as-synthesized $\mathrm{ZnO}$ products was observed by TEM. The TEM images, selected area electron diffraction (SAED) pattern of the $\mathrm{ZnO}$ nanowires, were obtained by using an accelerating voltage of $200 \mathrm{kV}$. It could be seen that when the obtained precipitate was under $200{ }^{\circ} \mathrm{C}$ hydrothermal reaction for $20 \mathrm{~h}$, the $\mathrm{ZnO}$ nanowires were obtained.

Table 5.1 Conditions of synthesizing $\mathrm{ZnO}$ nanorods/nanowires.

\begin{tabular}{|l|l|l|l|l|l|l|l|l|l|}
\hline Reaction time / h & \multicolumn{3}{|c|}{12} & \multicolumn{3}{c|}{16} & \multicolumn{3}{c|}{20} \\
\hline Temperature $/{ }^{\circ} \mathrm{C}$ & 160 & 180 & 200 & 160 & 180 & 200 & 160 & 180 & 200 \\
\hline
\end{tabular}

From the TEM image of the as-synthesized $\mathrm{ZnO}$ nanowires shown in Figure 5.1(c), most of the $\mathrm{ZnO}$ nanowires had diameters of 20-200 nm and lengths longer than $20 \mu \mathrm{m}$ (See Figure 5.2), indicating the aspect ratio was more then 100:1. These nanowires were relatively straight and their surfaces were very smooth. A typical SAED pattern (Figure 5.1(d)) taken from a single $\mathrm{ZnO}$ nanowire was indexed as a hexagonal structure of $\mathrm{ZnO}$ recorded from the $\left[\begin{array}{llll}1 & 0 & \overline{2} & 2\end{array}\right]$ zone axis, which indicated that the as-synthesized $\mathrm{ZnO}$ nanowires were similar in nature to single crystals. 

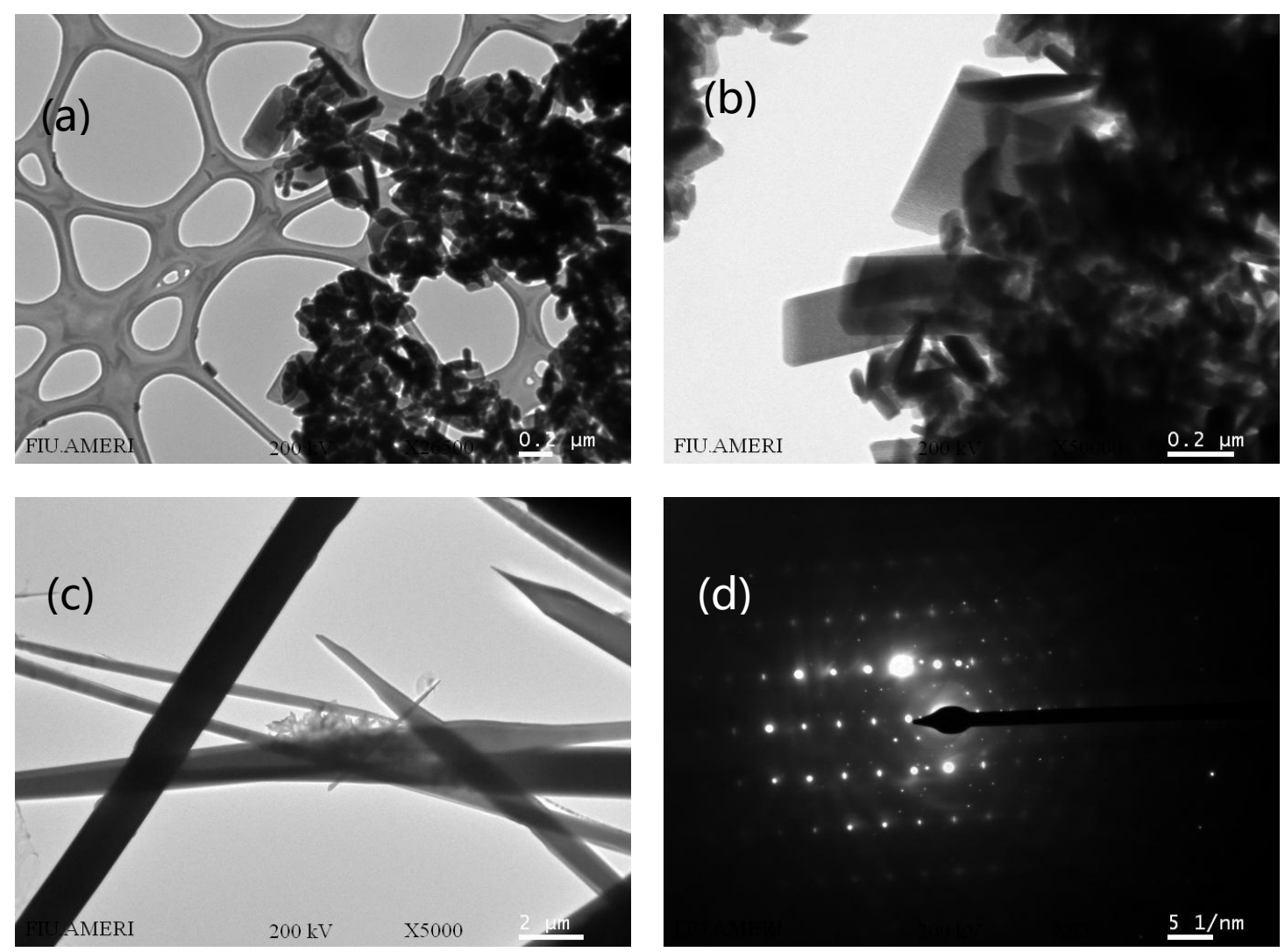

Figure 5.1 TEM image of the hydrothermal synthesized $\mathrm{ZnO}$ at (a) $160{ }^{\circ} \mathrm{C}$, (b) $180{ }^{\circ} \mathrm{C}$, (c) $200{ }^{\circ} \mathrm{C}$ for $20 \mathrm{~h}$ and (d) a typical SAED pattern of a single $\mathrm{ZnO}$ nanowire.
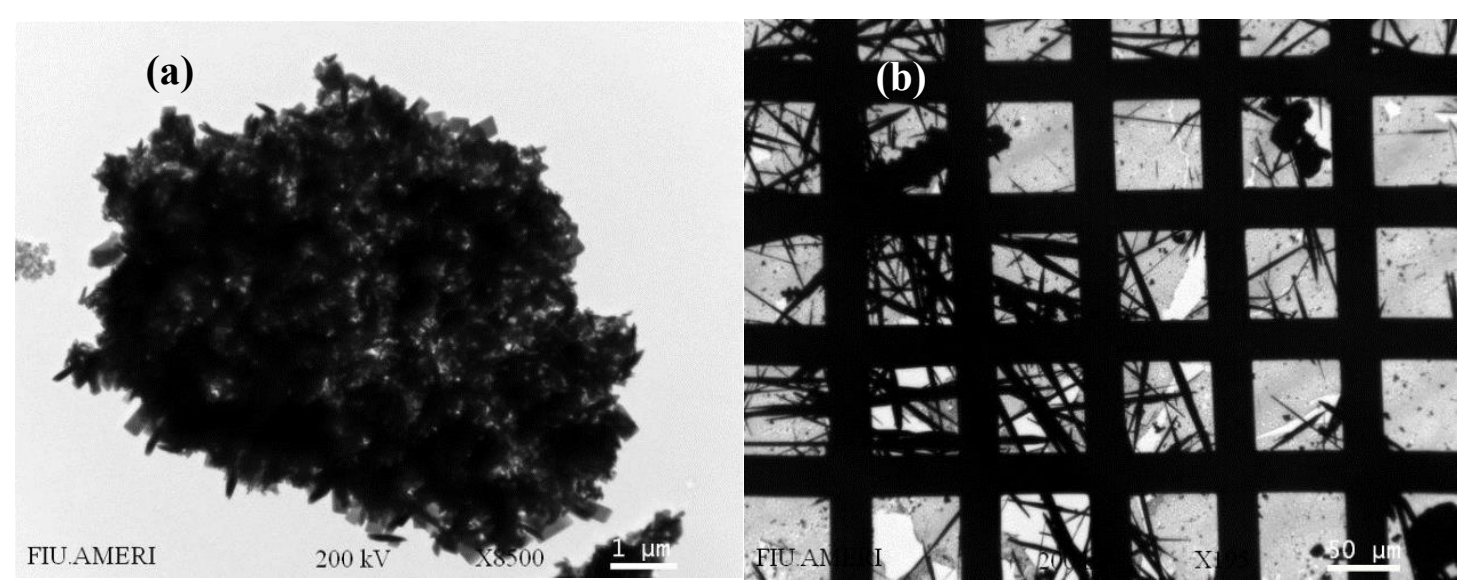

Figure 5.2 TEM images of the hydrothermal synthesized $\mathrm{ZnO}$ at (a) $160{ }^{\circ} \mathrm{C}$ and (b) 200 ${ }^{\circ} \mathrm{C}$ for $20 \mathrm{~h}$.

Also, the evolution of the products by changing the temperature and time of the hydrothermal reaction were studied. When the temperature was below $200{ }^{\circ} \mathrm{C}$, such as 
$160{ }^{\circ} \mathrm{C}$ or even $180{ }^{\circ} \mathrm{C}$, the $\mathrm{ZnO}$ particle did not grow into nanowires even if the other reaction conditions remained the same. The synthesized $\mathrm{ZnO}$ at $160^{\circ} \mathrm{C}$ for $20 \mathrm{~h}$ shown in Figure 5.1(a) and Figure 5.2(a) indicated that the $\mathrm{ZnO}$ have diameters of 12-25 $\mathrm{nm}$ and their lengths just about $0.2 \mu \mathrm{m}$.

Table 5.2 Diameters, length and aspect ratio of nano-ZnO in different synthesizing conditions.

\begin{tabular}{|c|c|c|c|}
\hline Synthesis temperature $/{ }^{\circ} \mathrm{C}$ & Diameter/nm & Length $/ \mu \mathrm{m}$ & Aspect ratio \\
\hline 160 & $12 \sim 25$ & $0.1 \sim 0.3$ & $3 \sim 6$ \\
\hline 180 & $20 \sim 100$ & $0.2 \sim 0.4$ & $5 \sim 10$ \\
\hline 200 & $20 \sim 1000$ & $20 \sim 200$ & $>100$ \\
\hline
\end{tabular}

\subsubsection{Dry Heat Treatment of MWCNT}

Dry heat treatment was performed for the refinement of MWCNT to improve its dispersion efficiency. About $8 \mathrm{~g}$ MWCNT was spread equally into a $2 \times 4 \mathrm{~cm}$ bottom of ceramic bowl. Then the bowl was put into a muffle furnace for 80 minutes with the temperature of $450{ }^{\circ} \mathrm{C}$ for the refinement.

5.2.1.3 Fabrication of PU/MWCNT, PU/ZnO and PU/MWCNT/ZnO nanowires films

Polyurethanes are the most commonly used viscoelastic materials for vibration control. Herein, PU was used as the polymer matrix due to their good adhesive properties, lack of by-products, and near-zero shrinkage during polymerization (Ishida 
and Allen, 1996). Piezoelectric $\mathrm{ZnO}$ nanowires and CNT were used both as the power generator and conductive component. Thus, an internal circuit was expected to be formed, which was expected to transform the electric energy into joule heating. The transformation of the vibration energy into Joule heat inside the composite was expected to cause damping of the vibration. By controlling the resistance of the composite, the damping process was expected to be enhanced (Uchino, 2000).

\subsubsection{Production of PU/DMF Solution}

Dimethylflouride (DMF) was used as dispersion solvent to produce dispersion solution (Rijn et al., 2004). Four gram of PU, $40 \mathrm{ml}$ DMF and one magnetic stir bar were put into $50 \mathrm{ml}$ conical flask. Then, the conical flask was put on the magnetic stirrer to stir for $24 \mathrm{~h}$ at $80^{\circ} \mathrm{C}$.

5.2.1.5 Production of PU/MWCNT, PU/ZnO Nanowires and PU/MWCNT/ZnO Nanowires Films

Four $\mathrm{ml}$ PU/DMF was put into a $15 \mathrm{ml}$ glass tube. Sixteen $\mathrm{mg}$ of well treated MWCNT (ZnO nanowires, or both 16mg MWCNT and 16mg ZnO nanowires) were added into it. The well-sealed tube was treated by ultrasonic waves for $3 \mathrm{~h}$ under normal temperature. Then, a homogenizer was used to mix them further for 1 hour at room temperature at a mixing speed of $30,000 \mathrm{rpm}$. The mixed solution was poured immediately into a silicone oil pretreated $2.5 \times 2.5 \mathrm{~cm}$ glass mold and then put into 110 ${ }^{\circ} \mathrm{C}$ oven for $2 \mathrm{~h}$ to evaporate the DMF. After cooling down, the films were peeled off the glass mold very carefully. Thus, the PU/MWCNT, PU/ZnO nanowires and 
$\mathrm{PU} / \mathrm{MWCNT} / \mathrm{ZnO}$ nanowires films were produced. The thickness of the produced films was about $150 \mu \mathrm{m}$.

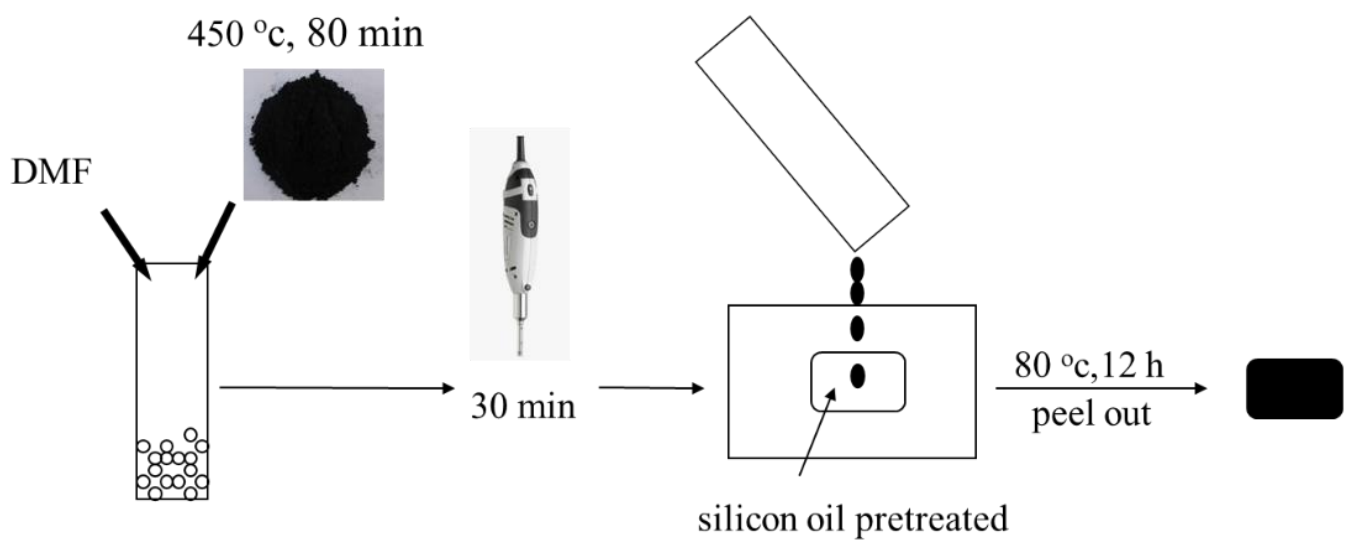

Figure 5.3 Procedures of making PU/MWCNT/ZnO nanorods/nanowires composites films.

5.2.2 Binding Layer Method to Make PU/MWCNT/ZnO Nanorods/Nanowires Films

The procedures to make PU/MWCNT was the same as Section 5.2.1.5. Pure PU layer was made by the following procedure: Four gram of PU, $40 \mathrm{ml} \mathrm{DMF}$ and one magnetic stir bar were put into $50 \mathrm{ml}$ conical flask. Then, the conical flask was put on the magnetic stirrer to stir for $24 \mathrm{~h}$ at $80{ }^{\circ} \mathrm{C}$. The solution was poured immediately into a silicone oil pretreated $2.5 \times 2.5 \mathrm{~cm}$ glass mold and then put into $110{ }^{\circ} \mathrm{C}$ oven for $2 \mathrm{~h}$ to evaporate the DMF. After cooling down, the films were peeled off the glass mold very carefully. Hence, the pure PU film was produced. The PU/MWCNT/ZnO nanorods composite films were made by attaching the PU layer to the Al base beam first, and then the PU/MWCNT layer was attached upon the PU layer. Finally, the MWCNT/ZnO nanorods were poured upon the PU/MWCNT layer (see Figure 5.4). Then, the 
composites films were produced by putting the previous combination of layers into a $110^{\circ} \mathrm{C}$ oven for two hours.

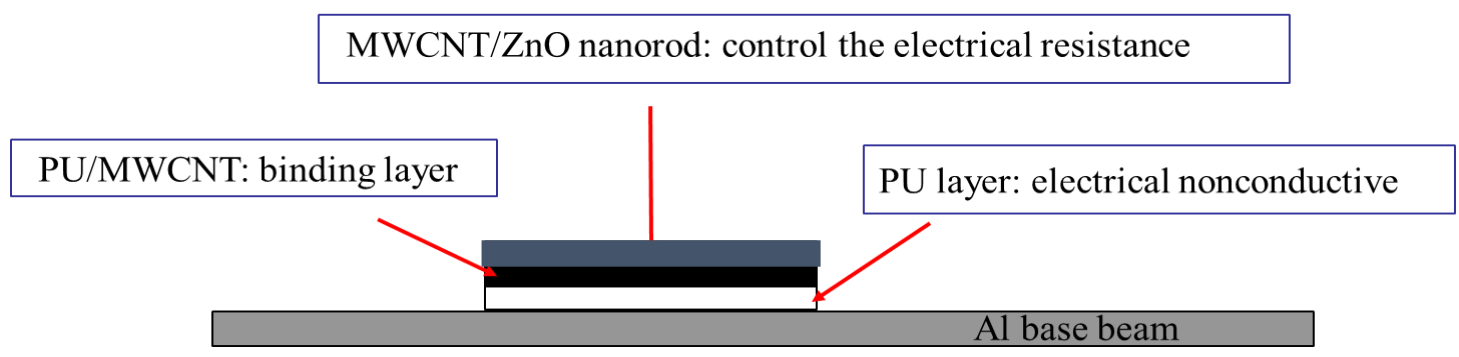

Figure 5.4 Schematic diagram of make PU/MWCNT/ZnO nanorods composites films.

\subsubsection{Oriented Method to Make PU/MWCNT/ZnO Nanorods/Nanowires Films}

As Figure 5.5 shows below, MWCNT and $\mathrm{ZnO}$ nanorods were brushed upon pure PU film, which was previously attached on to the Al base beam. With this fabrication method, we expected to evaluate the properties of oriented nanomaterials such as MWCNT and ZnO nanorods/nanowires and how they performed in composites films.

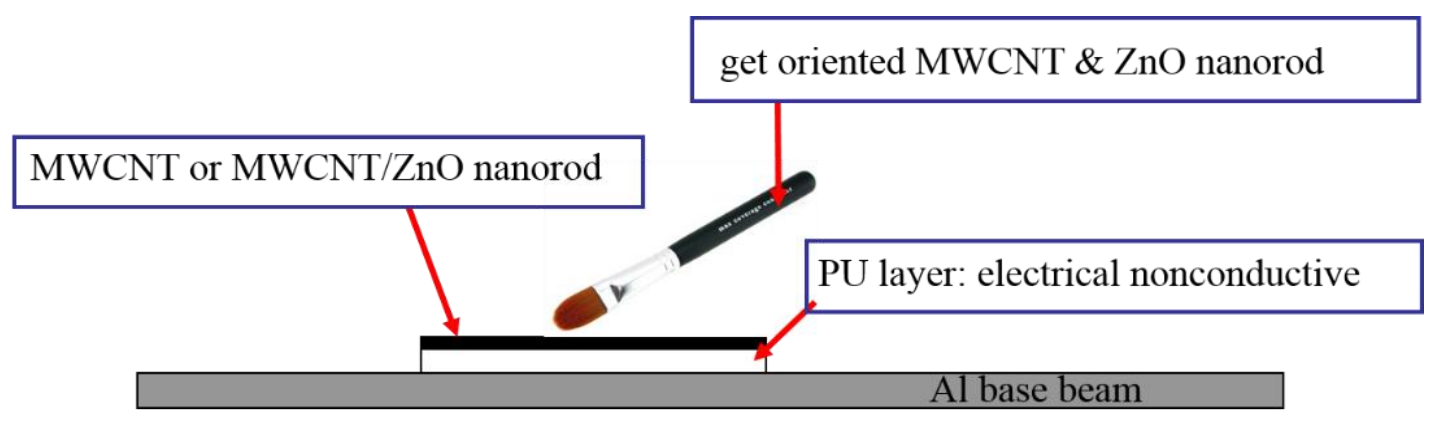

Figure 5.5 Schematic diagram of make PU/MWCNT/ZnO nanorods composites films. 


\subsubsection{Problems Encountered with Those Methods}

\subsubsection{Disadvantages of Mold Casting method}

Mold casting method used to make PU/MWCNT/ZnO nanorods composites films was ideally expected to produce an internal circuit. Then, it was expected that any change in the electric energy of the composite would be converted into joule heating which would enable this composite to have vibration control properties. Though the composites films made by this method showed damping properties could be achieved, such films were found not to have both damping and strain sensing properties.

Firstly, in order to test the sample using both the free vibration test method and the static and dynamic strain sensing, it was necessary that the PU/MWCNT/ZnO nanorods/nanowires composites films be attached to the Al base beams. To avoid the effect of the electric conductive properties of the Al base beam, non-conductive adhesive was used to attach the $\mathrm{PU} / \mathrm{MWCNT} / \mathrm{ZnO}$ nanorods/nanowires composites films to the $\mathrm{Al}$ base beam, as shown in Figure 5.6. However, PU/MWCNT/ZnO nanorods/nanowires composites films were found to react with the non-conductive adhesive causing the sample to curl and lose full contact with the Al base. This effectively nullified the sample's ability to sense strain changes.

Secondly, the PU/MWCNT/ZnO nanorods/nanowires composites films that were made were expected to have a suitable electrical resistance, which was necessary for the sample to be used as a strain sensor. However, our PU/MWCNT/ZnO nanorods/nanowires composites films displayed too large a value of electrical resistance for a strain sensor. This is because the PU is an electrical non-conductive 
material and the combination of the PU/MWCNT/ZnO is dominated by the PU causing the value of the electrical resistance of the film to be too high.

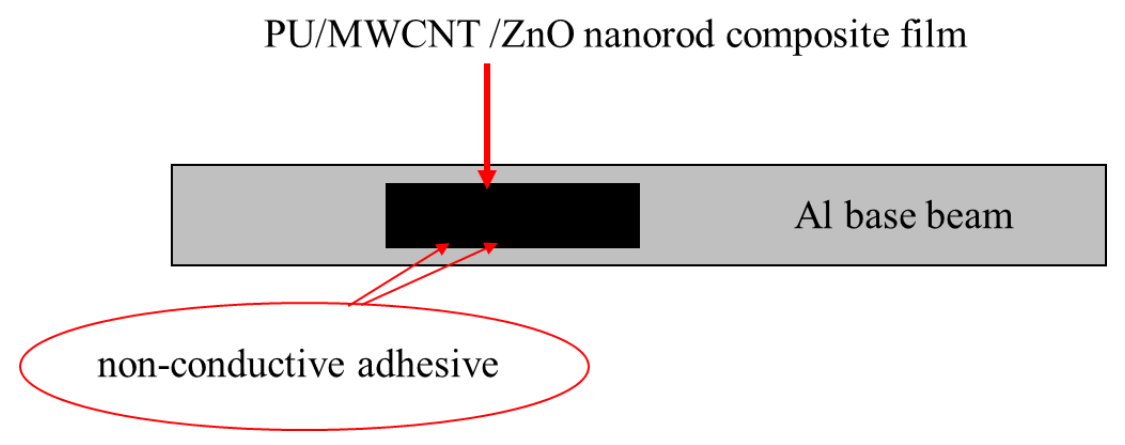

Figure 5.6 Schematic image of PU/MWCNT/ZnO nanorods/nanowires composites films attached upon Al base beam with non-conductive adhesive.

\subsubsection{Disadvantages of Binding Layer Method and Oriented Method}

Because of the disadvantages in using the mold casting method, a new methodology was used to make PU/MWCNT/ZnO nanorods/nanowires composites films, namely, the binding layer method and oriented method were attempted to make the composite film, the details of these methods are found in section 5.2.2 and 5.2.3. Also, an attempt was made to use pure PU layer to replace the non-conductive adhesive as a means of binding the sample to the Al base beam. This attempt also did not work.

We also tried to place the MWCNT (or/and $\mathrm{ZnO}$ nanorods) upon the film to avoid the electrical non-conductive problem we encountered with the mold casting method. However, there were technical difficulties to do this. So, a layer by layer attachment method was employed and is explained in the following sections. 


\subsubsection{Layer by Layer Attachment Method to Prepare Pu/Buckypaper}

Buckypaper was bought from Nano-lab (Newton, MA, www.nano-lab.com). The procedures Nano-lab provides to make Buckypaper is briefly summarized in the following paragraph.

MWCNT was purified by placing into the mixed acids (hydrochloric acid $(\mathrm{HCl})$ and nitric acid $\left.\left(\mathrm{H}_{2} \mathrm{SO}_{4}\right)\right)$ to remove the catalyst iron particles. The MWCNT were then washed with deionized water until they were neutralized. Then, the material was filtered, dried and reground into powder. Nanosperse AQ, which is a surfactant was added into MWCNT to form a stable suspension. Finally, the suspension was pressed through a filter, resulting in the MWCNT as residual film on the filter surface. The Buckypaper was obtained when peeled from the filter surface. The Buckypaper was made of hollowstructured MWCNT with 15 $\pm 5 \mathrm{~nm}$ outer diameter, about 5-20 microns in length and purity higher than $95 \%$. The thickness of the MWCNT film was around 100 microns and the relative density was about $50 \%$.
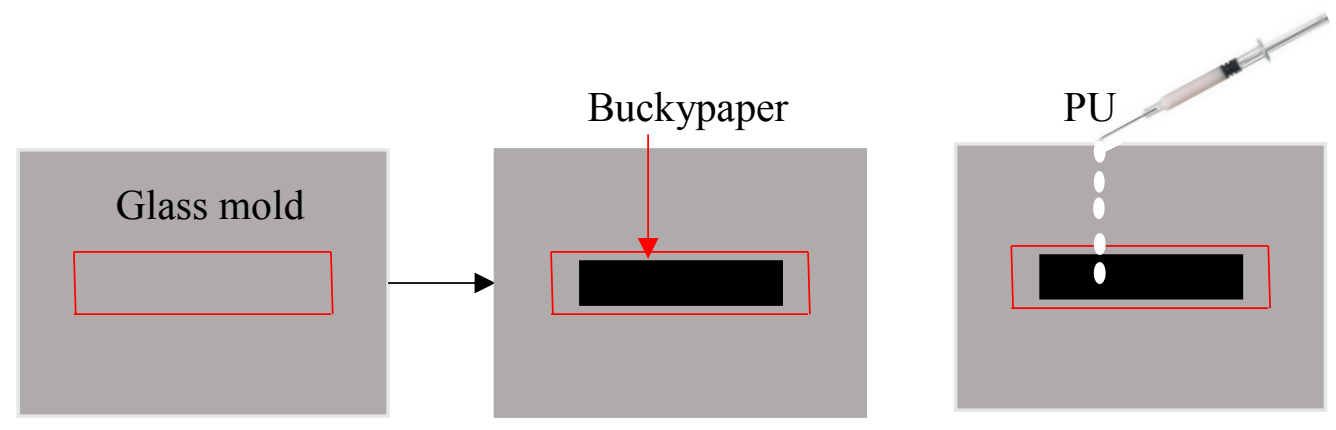

Figure 5.7 The process for preparation of PU/Buckypaper.

The Buckypaper received from Nano-lab was cut into $3.5 \mathrm{~cm} \times 0.9 \mathrm{~cm} \times 100 \mu \mathrm{m}$ sections and placed into $6 \mathrm{~cm} \times 1.3 \mathrm{~cm} \times 0.3 \mathrm{~cm}$ glass mold very carefully so that there was no space between the mold and the Buckypaper. 
Four grams PU were dissolved into $50 \mathrm{ml}$ DMF. Four ml of PU/DMF were cast in the Buckypaper filled glass mold. Then, the mold was put in $100^{\circ} \mathrm{C}$ oven to dry for 5 h. After that, the PU/MWCNT composites were obtained by peeling them off the glass mold. The thickness of the PU is about $80 \mu \mathrm{m}$. So, the total thickness of the PU/MWCNT is around $180 \mu \mathrm{m}$. The process is briefly shown in Figure 5.7.

\subsubsection{Sample Setup for Free Vibration Tests}

\subsubsection{Single Side Attachment Mode}

The first single side attachment model was aimed to evaluate the differences in performance among pure PU, Buckypaper and PU/Buckypaper composites. Firstly, 3.5 $\mathrm{cm} \times 0.9 \mathrm{~cm}($ length $\times$ width $)$ of pure PU, Buckypaper and PU/Buckypaper with $80 \mu \mathrm{m}$, $100 \mu \mathrm{m}$ and $180 \mu \mathrm{m}$ in thickness, respectively, were attached to $9.5 \mathrm{~cm} \times 0.9 \mathrm{~cm} \times$ $0.048 \mathrm{~cm}$ aluminum base beam single side by a thin layer of adhesive (see Figure 5.8). Three very thin layers of nonconductive 3M CA100 liquid instant adhesive were used to cover these samples to separate the samples from the environment. Each specimen was dried at room temperature for $24 \mathrm{hrs}$. The base beam material used was 6061-T6 Aluminum.

The red dashed lines shown in Figure 5.9 defined the two possible locations where the beam was clamped. The side of the Aluminum base beam without the attachment of samples were tested then cut $1 \mathrm{~cm}$ at a time and the results were compared. The unclamped side was then made to vibrate and the damping ratio was determined. 


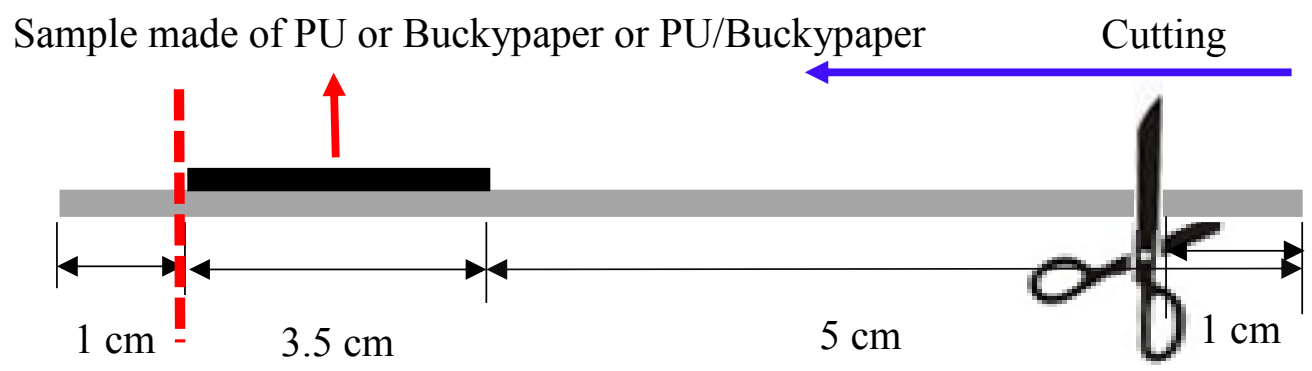

Figure 5.8 Schematic images of single side attachment. The dimension of samples, Al beams and the locations where samples were attached, the locations (red line) where the beam was clamped and the directions and lengths for beam removal.

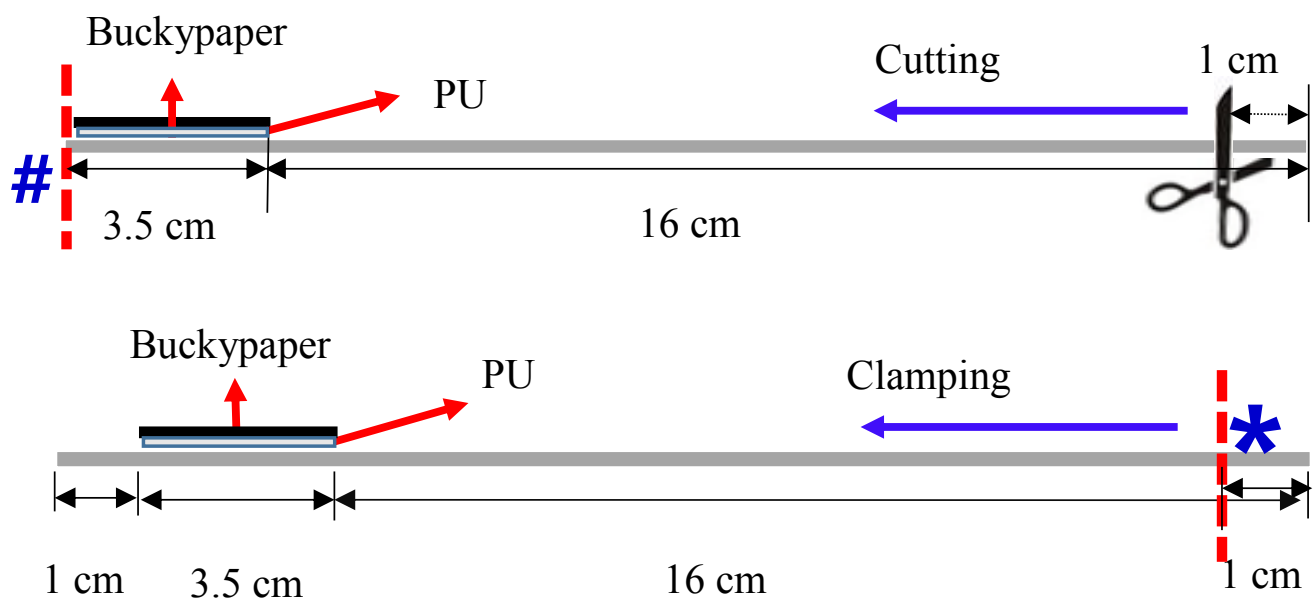

Figure 5.9 Schematic images of single side's attachment. The dimension of samples, Al beams and the locations where samples were attached, the locations of sample side \# and free end side * where the beam was clamped and the directions and lengths for both beam removal and clamping.

The second single side attachment model was built to investigate how the damping changed as a function of the coverage of PU/Buckypaper to aluminum base beams. The PU/Buckypaper with dimension of $3.5 \mathrm{~cm} \times 0.9 \mathrm{~cm} \times 0.018 \mathrm{~cm}$ was attached upon a $20.5 \mathrm{~cm} \times 0.9 \mathrm{~cm} \times 0.048 \mathrm{~cm}$ Aluminum base beam on one side (see Figure 5.9) using a thin layer of glue as discussed in the previous paragraph. The specimen was dried at 
room temperature for $24 \mathrm{hrs}$. Free vibration comparisons were made by using either the sample side, \#, or the uncovered side, *, alternately, as the fixed support for the cantilever beam. The red dashed lines shown in Figure 5.9 defined the locations where the beam was clamped. The side of the Al base beam without the attachment of samples were either cut or clamped $1 \mathrm{~cm}$ at a time until the clamp was next to the sample. The unclamped side was then made to vibrate and the damping ratio was determined and the results were compared.

\subsubsection{Both Sided Attachment Mode}

All the dimensions of sample and aluminum base beams, the clamped locations and the directions and lengths for both cutting and clamping were kept the same as the second single side attachment mode described above. The only change that was made was that the aluminum base was covered on both sides by the sample (See Figure 5.10).
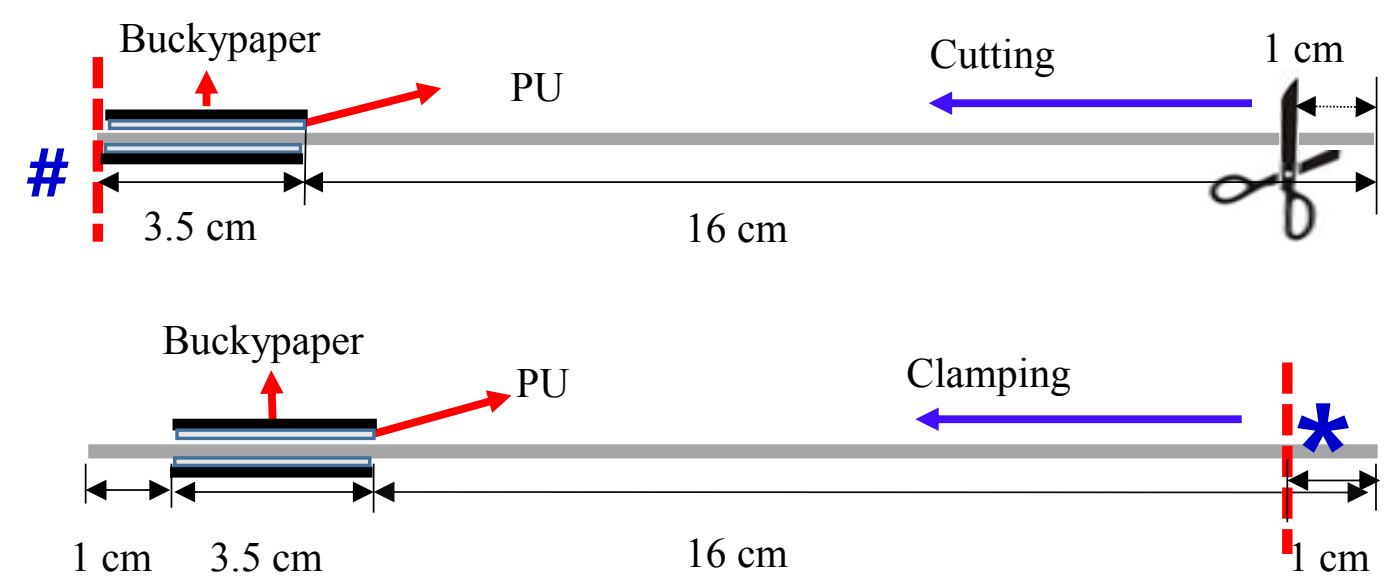

Figure 5.10 Schematic images of double sides' attachment. The dimension of samples, Al beams and the locations where samples were attached, the locations of sample side \# and free end side * where the beam was clamped and the directions and lengths for both beam removal and clamping. 


\subsubsection{Damping Test Methodology}

The cantilever beams of Figures 5.8, 5.9 and 5.10 were given the same, small initial displacement and then released for free vibration. Unclamped end displacements were measured by a laser vibrometer and a two channels digital oscilloscope was used display and store the signal. Five trials were made for each setup and the mean value of the damping ratio was calculated. This ratio can be related to the logarithmic decrement of consecutive maxima of end displacements and can be correlated to how much vibration energy is removed from the sample. The higher the damping ratio, the better the sample acted as a damper.

\subsubsection{Sample Setup for Drift, Static Tensile Test, and Dynamic Tests}

The sensor was bonded to the center of a 50-mil thick 6061-T6 Al rectangular bar using 3M CA100 liquid instant adhesive. Each specimen was held in place by a weight and dried in room temperature for $24 \mathrm{hrs}$. The bonding area was prepared to ensure a smooth surface, as is normally defined for a foil strain gage, with no oxide formations on the surface. A foil strain gage, Omega SGD-30/350-LY40, $3 \mathrm{~cm}$ long x $0.3 \mathrm{~cm}$ wide, was bonded to the opposite side of the substrate. 24 AWG solid wire was used to connect decade resistor boxes manufactured by Eisco Labs in a Wheatstone bridge setup for the sensor or the foil strain gauge. Prior to testing, resistance of the sensor and strain gage was measured and the corresponding decade resistance boxes were set to match. The decade resistor boxes have a resolution of about $1 \mathrm{Ohm}$. This causes the initial millivolt potential difference to rarely start at zero. To compensate for this, where 
appropriate, data points were shifted to allow for comparison of different sensors. Constant voltage was provided during testing by B\&K precision power supply (1745A). The voltage supply was set to 5 volts for measured resistance above 20 Ohms. For resistance below $20 \mathrm{Ohms}$, the voltage supply was set to 2 volts. Signals from the drift and strain measurement tests were collected using National Instruments NI cDAQ-9172 board and LabView software. Signals from the Dynamic sensing tests were collected using Data Translation DT9837C and SpectraPLUS-DT. Linearity and sensitivity of the sensor were evaluated using quasi-static loadings. These loadings were carried out using a United SSTM-2K model tensile test machine.

\subsubsection{Drift Test Procedures}

The mounted sample was connected to the measurement system and placed in a no load and ambient temperature condition for at least 24 hours prior to testing. The test equipment was then turned on, and at least 30 minutes of data were collected for each sample. The drift test was performed several times for the sake of repeatability.

\subsubsection{Static Test Procedures}

The mounted sample was placed in the tensile testing machine and connected to the measurement system. The system was left idle for at least 1 hour prior to testing to remove any accumulated stresses due to mounting. Signal collection was initiated. The tensile test system was set to extend at a rate of $0.001 "+/-0.0005$ " per minute. This way the system was operating quasistatically. The system was set to increase ramp force at the set extension rate until $400 \mathrm{lbs}$. of force was achieved. The system was then set to 
instantaneously reduce load to $0 \mathrm{lbs}$. This procedure was repeated multiple times to determine repeatability of the results and hysteresis in the system.

\subsubsection{Dynamic Test Procedures}

The sample was mounted onto a cantilever beam system. The sensor was located as close as possible to the fixed end. The free end was attached to a vibration drum that was vibrated at specified frequencies. An accelerometer was attached to the free end, as close to the center of the vibration drum as possible. The accelerometer linked into a feedback loop that ensured that the acceleration of the free end was correct according to the frequency versus acceleration profile. This profile was set up such that the acceleration started at $10 \mathrm{~Hz}$ and $10 \mathrm{~g}$ 's, ramped up to $20 \mathrm{~Hz}$ and $20 \mathrm{~g}$ 's, then held a constant acceleration of $20 \mathrm{~g}$ 's from $20 \mathrm{~Hz}$ to $6000 \mathrm{~Hz}$. The control system was then set to run a specified frequency or the system was swept across a range of frequencies. The resulting signals were collected and analyzed using the Hanning FFT algorithm. The peak hold data for the signals were collected over the course of 500 signal samples using a sampling rate of $11000 \mathrm{~Hz}$ for an input frequency range of 10 to $1000 \mathrm{~Hz}$ and $60000 \mathrm{~Hz}$ for an input frequency range of 1000 to $5000 \mathrm{~Hz}$. Five sets of peak hold data were taken and analyzed.

\subsection{Results and Discussion}

\subsubsection{Damping Ratios of PU, Buckypaper and PU/Buckypaper Composites}

The free end vibration test has already shown that the Buckypaper has strain sensing properties (Buffa et al., 2007). Also, PU has been broadly studied because it 
has excellent damping ability. Herein, the free end vibration test method was adopted to study the damping behaviors of PU, Buckypaper and their layer by layer PU/Buckypaper composite. For the Al base beam the single side attachment free vibration trial results are shown in Figure 5.11 (see Figure 5.8 for the configuration of the tests setup). Many tests were run and no delamination in the PU/Buckypaper composites was observed in any of the specimens used.

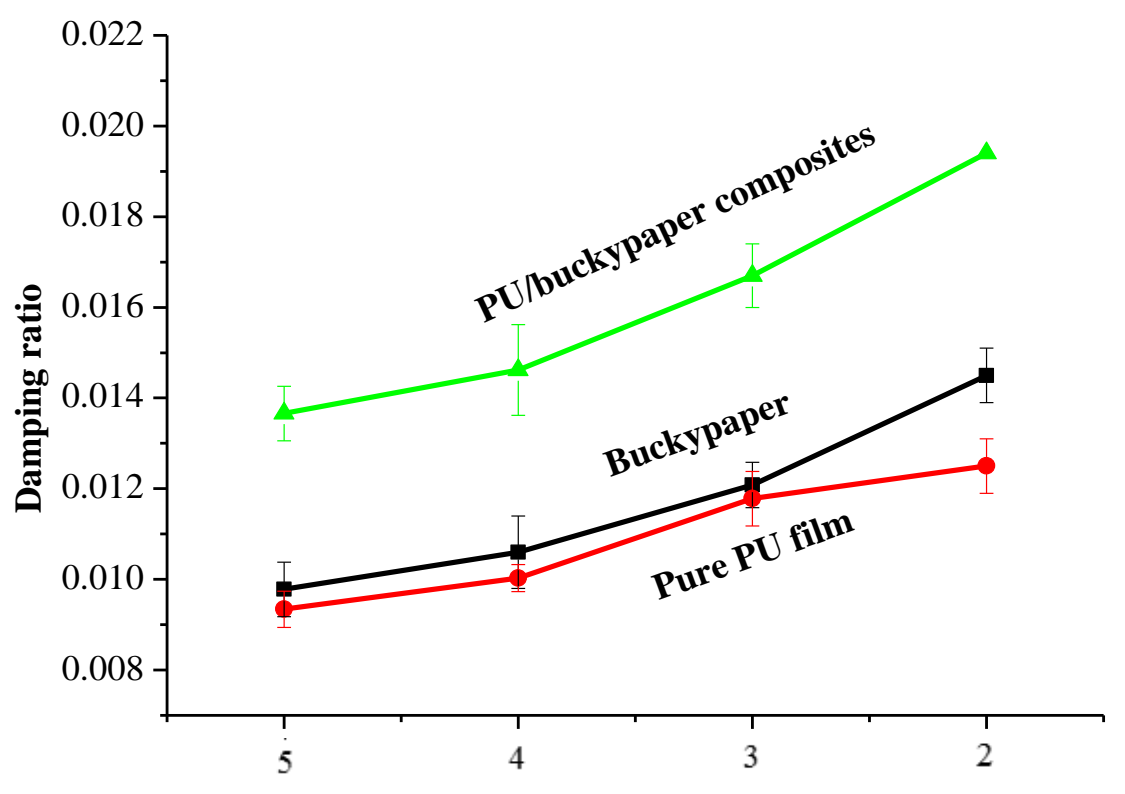

Distance from the free end to the sample $/ \mathrm{cm}$

Figure 5.11 Damping ratios comparison of PU, Buckypaper and PU/Buckypaper composites. Error bars are shown for each sample at each beam length.

From Figure 5.11, PU/Buckypaper sensors have higher damping ratios than their individual components (pure PU film and Buckypaper) in different lengths of Al base beams. Also, with the length of the beam decreased, the damping ratios of pure PU film, Buckypaper and their combination PU/Buckypaper film increased. In other word, with the beam coverage increased, the damping ratios of pure PU film, Buckypaper and their 
combination PU/Buckypaper film increased. The differences of damping ratios between pure PU and Buckypaper are not distinguished for with the four different coverage of the Al base beam.

\subsubsection{Damping Results for Single Side Attachment}

As mentioned previously, the reason for the creation of the composite was for use as a sensor-actuator with damping capabilities. To check on the damping capability aspect, several tests were performed. For the Aluminum base beam, single sided attachment test (see Figure 5.9 for the configuration of the tests setup), free vibration trial results are shown in Figure 5.12.

Five trials were run for each of clamping conditions listed. The results clearly showed that the damping ratio initially increased as the distance from the free end to the sample decreased for both clamping on the free end side * and clamping on the sample side \# (see Figure 5.12). Then, it decreased as the distance from the free end to the sample further decreased for both clamping on the free end side * and clamping on the sample side \#. For both clamping on the free end side * and clamping on the sample side \#, the damping ratio reached the highest values 0.0124 and 0.0202 , respectively, when the distance from the free end to the sample was $6 \mathrm{~cm}$. Comparing the highest damping ratios, 0.0124 and 0.0202 , when the distance from the free end to the sample was the same $6 \mathrm{~cm}$, it was determined that clamping on the sample side \# was $162.9 \%$ higher than when clamping on the free end side *. The trends were found to follow previous published results for constrained layer viscoelastic dampers. 

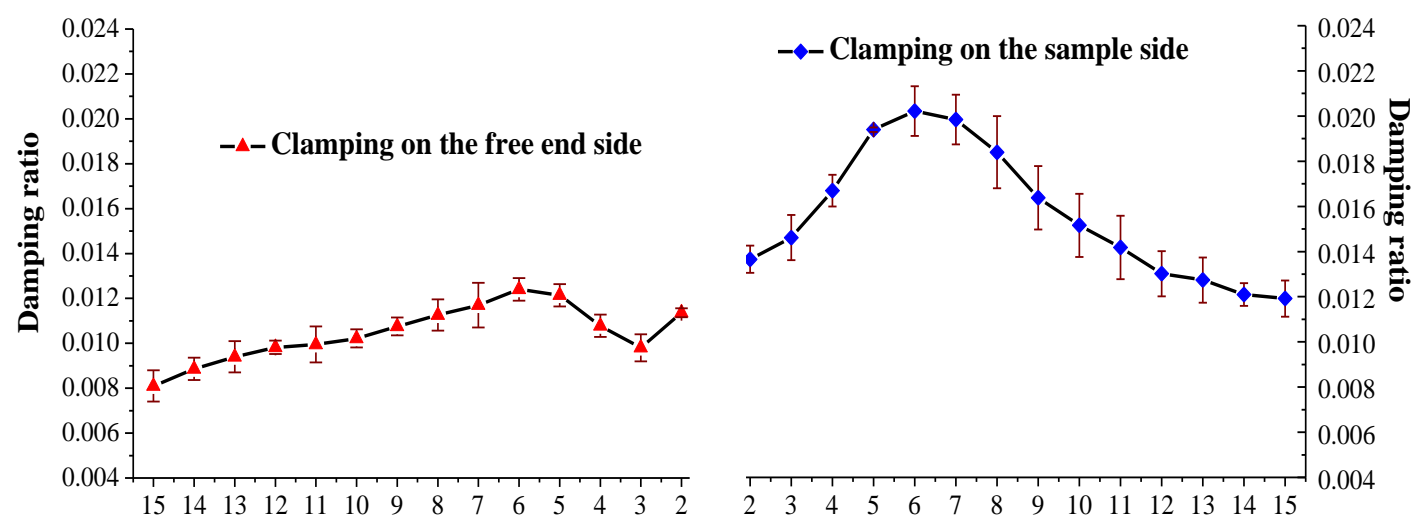

Distance from the free end to the closest side of the sample $(\mathrm{cm})$

Figure 5.12 Damping ratios of single side attached PU/Buckypaper clamped on the free end $*$ side and the sample \# side. Error bars are shown for each result shown.

There was a further increase of the damping ratio after it decreased for the case where the clamping was on the free end side *. This was reasonable when compared with the trend of the damping ratio of clamping on the sample side. The damping ratios remained lower for each new beam length trial when clamped on the free end side compared with the value for each beam length trial when clamped on the sample side. However, the damping ratios had to be the same when the beam being tested became symmetric with respect to the vertical axis; meaning, it didn't matter whether you tested the clamped side or the free end side, both had to be the same configuration leading to the same damping ratio.

\subsubsection{Damping Results for Single Side Attachment}

The double sides' attachment setup which is shown in Figure 5.10 displayed the same trend of damping ratios as single sided attachment (See Figure 5.13). However, the double-sided attachment had higher damping ratios compared with single side 
attachment. It reached the highest damping ratio of 0.033 with double sides' attachment when the sample's right end was $3 \mathrm{~cm}$ away from the free end. Also, the damping ratio differences between clamping on the sample side and clamping on the free end with the same distance from the free end to the sample was bigger for double sided attachment than for the single sided attachment. An increase in damping ratio when clamped on the free end side was noted, which was just like the single side attachment trend. The Ycoordinate value was very close at the 0 -cm value of $\mathrm{X}$-coordinate, which was expected.

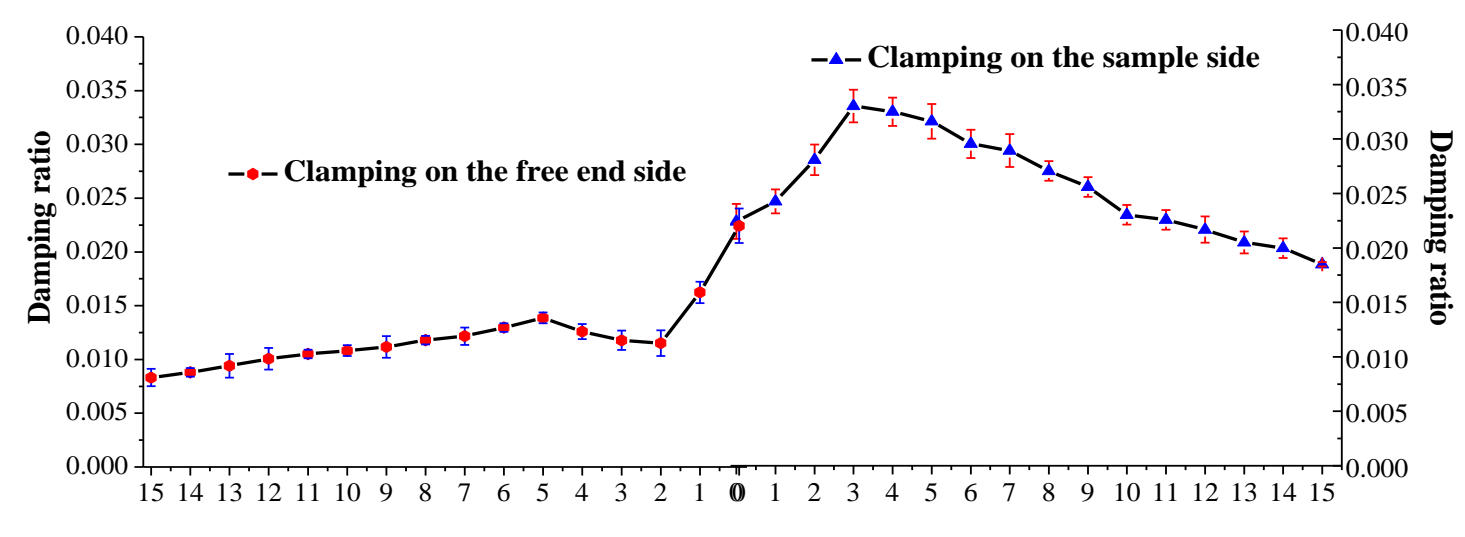

Distance from the free end to closest side of the sample $(\mathrm{cm})$

Figure 5.13 Damping ratios of double sides attached PU/Buckypaper clamped on the uncovered * side and sample \# side. Error bars are shown for each result shown.

When one compared the loss factor as a function of the non-dimensional coverage length in both these cases, the graphs of Figure 5.14 are obtained. Here, the loss factor, $\eta$, is twice the damping ratio for small damping ratios up to 0.3 (Li et al., 2014; Rao, 2004). Similarities were noted in trends of the loss factor for the sample side clamping condition, \#, when compared to Chen and Levy (1996). First the double-sided coverage produced a higher loss factor which increased as the sample length to the beam length 
$(\mathrm{L} 1 / \mathrm{L})$ increased and then the loss factor began to decrease as beam coverage, $\mathrm{L} 1 / \mathrm{L}$ tended to 1 .

An analysis of the loss factor versus non-dimensional coverage length when clamping was on the free end side, $*$, is also undertaken. This analysis was found to apply for the single side and for the double side coverage, as well. The loss factor for small $\mathrm{L} 1 / \mathrm{L}$ is best be described as that for a sample starting to act as a passive layer type damping element with an end mass. But as the beam material was removed, thereby increasing $\mathrm{L} 1 / \mathrm{L}$, the loss factor of the sample transitioned to more of a loss factor for a passive layer type damping element covering more and more of the beam. We noted that the loss factor, $\eta$, first increased with coverage length, $\mathrm{L} 1 / \mathrm{L}$, though its value was several times smaller than its value when the beam was clamped on the sample side. In this configuration, the sample acted as a small mass at the end of the beam and coverage length effects dominated. Then the loss factor was found to decrease to a minimum value before increasing again as $\mathrm{L} 1 / \mathrm{L}$, the dimensionless coverage length, increased. This decrease was probably due to the fact that as more beam was removed; the sample started to act as a larger end mass compared to the mass of the beam and that becomes the dominating effect. Such a system would tend to execute relatively larger end displacements, and, according to Rao (2004), for viscoelastic materials, this would imply a lower loss factor (see Rao (2004)). As more beam material was removed, a second increase occurred in the loss factor due to the sample transitioning from being like an end mass to beginning to cover most the beam. This behavior was like that found in Chen and Levy (1996) for the vibration of a viscoelastic constrained layer damping 
of a beam with end mass. However, this increase-decrease-increase phenomenon in the loss factor was noticed for both the single side and double side coverage of the beam. Though Chen and Levy $(1994,1996)$ investigated the change in L1/L by increasing L1 and keeping L fixed, the behavior seen in this investigation can be deduced from the results found in (Chen and Levy, 1996; Levy and Chen, 1994) and follow the trends therein. However, the differences in loss factor between single sided and double sided coverage were much larger in (Chen and Levy, 1996) compared to what was noted here because of the materials used. Yet, the trends in (Chen and Levy, 1996) and shown in this work were very similar.

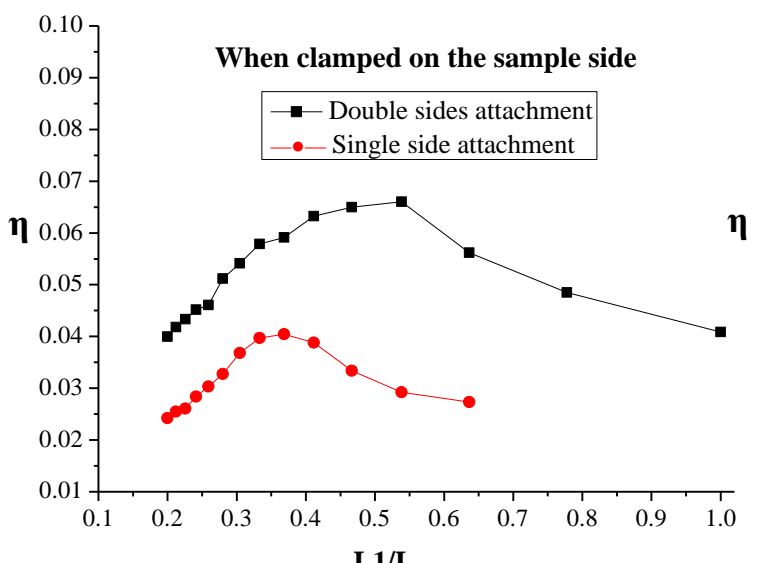

L1/L

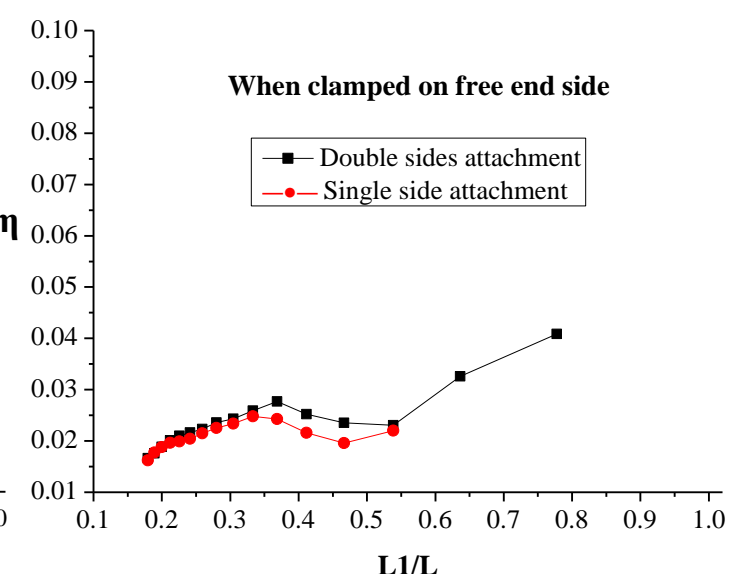

$\mathbf{L} 1 / \mathbf{L}$

Figure 5.14 Comparison between single side and double sided attachment of the PU/Buckypaper sample for both clamping conditions.

\subsubsection{Static Sensing Properties}

\subsubsection{The Drift Effect}

The stability of each sensor was tested and typical results for some samples and strain gage are shown below. The drift test was executed for the sample and for the 
strain gage for a period of 30 minutes or more. The voltage data were captured and for the sensors were shifted so that average recorded signal value was zero. This allowed for direct comparison of the sensors on the graph. The change in voltage per hour for typical results are also tabulated below. The correlation of the data was small because significant noise was generated in the results due to the high sampling rate. Therefore, other measurements given in succeeding sections do not include the correlation values.

Table 5.3 Trend line slopes and R-values.

\begin{tabular}{|l|c|c|}
\hline Type of Sample & $\Delta \mathrm{mV} / \mathrm{hour}$ & $\mathrm{R}^{2}$ \\
\hline PU/Buckypaper-blue line & $-0.6184 \mathrm{mV} / \mathrm{h}$ & 0.0353 \\
\hline PU/Buckypaper-black line & $-1.7925 \mathrm{mV} / \mathrm{h}$ & 0.0437 \\
\hline Strain Gauge-green line & $0.0866 \mathrm{mV} / \mathrm{h}$ & 0.0003 \\
\hline
\end{tabular}

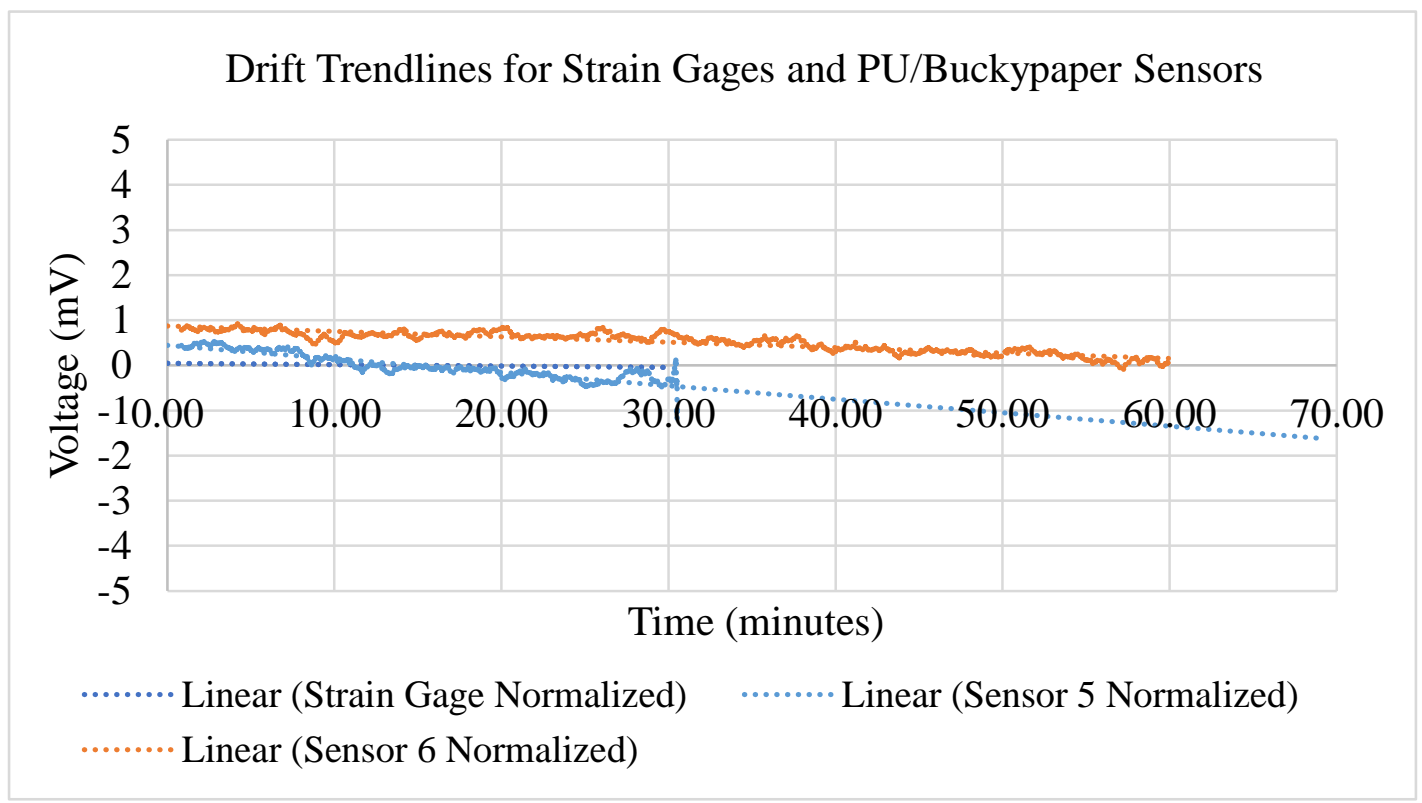

Figure 5.15 (a) Drift trendlines for typical PU/Buckypaper sensor and strain gage. 


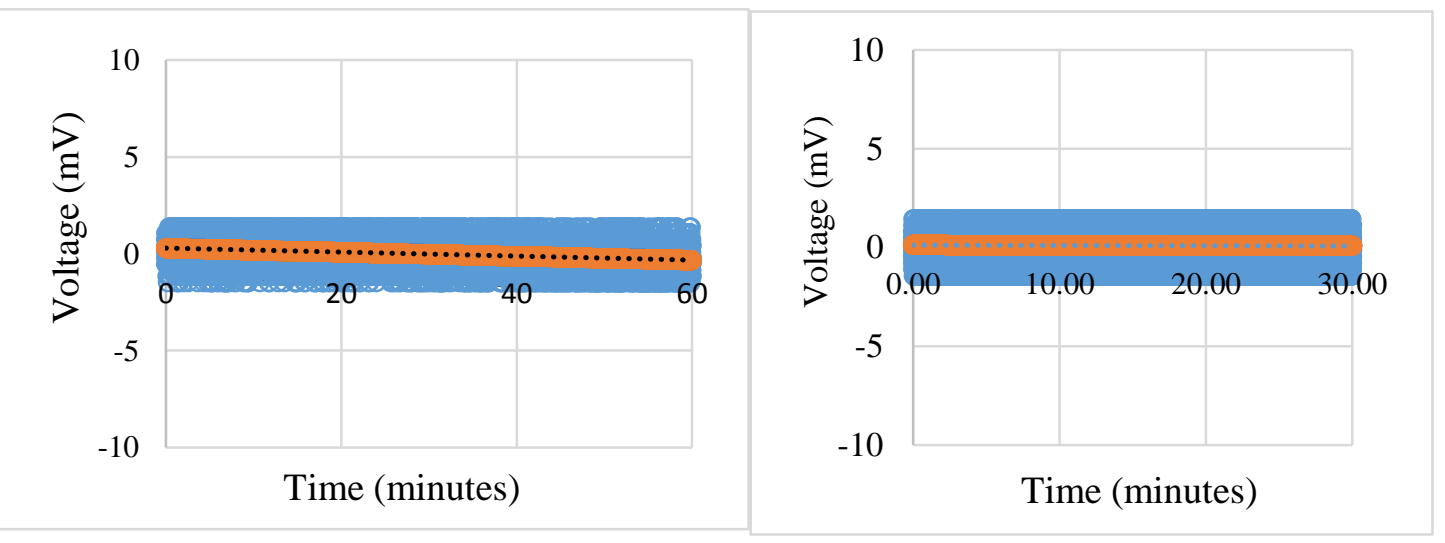

Figure 5.15 (b) Drift test for PU/Buckypaper sample (sensor 5) (left) and (c) drift test for strain gage (right).

Figure 5.15 (a) shows typical results for one strain gage and two sample sensors. The strain gage showed almost no changes over the test period though there was a slight downward slope to the line. The sample sensors also exhibited very small slopes as well. Figures 5.15 (b) and 5.15 (c) show actual traces for one of the samples and the strain gage where the sensor voltage changed by $-0.6184 \mathrm{mV} / \mathrm{hr}$ while the foil strain gage changed by $-0.0866 \mathrm{mV} / \mathrm{hr}$. The results of this testing showed that some samples were unstable and had significant drift while others were much more stable but consistently had a drift that was one order of magnitude larger than that of the strain gage. This was reasonable since our testing apparatus could not be moved and had no way of isolating our samples from outside vibrations. Foil strain gages were consistent and are known to be stable in this type of environment.

\subsubsection{Linearity and Repeatability - Static Tensile Test}

Linearity and repeatability are two very important characteristics for strain sensors. It is desirable that the relationship between the applied strain and the output voltage be 
linear. The source voltage for the sensor and the foil strain gage in Figure 5.16 are equivalent.

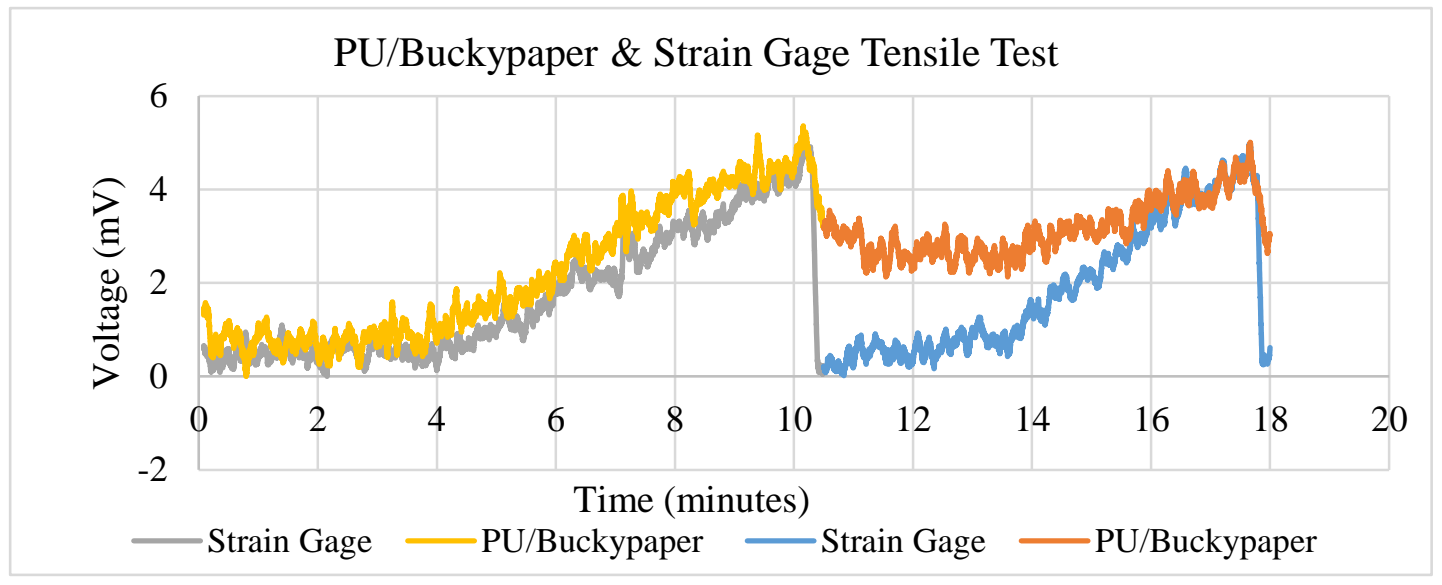

Figure 5.16 Raw data of repeat loading comparison of PU/Buckypaper and strain gage.

Figures 5.16-5.18 shows typical data for a specific sample that responded as desired. This specific dataset were a result of two quasi-static loadings and instantaneous unload cycles. Figure 5.16-5.18 provides typical data obtained from testing both the sample sensor and the strain gauge. Provided are the results of the first tensile test loading in which the strain sensing behavior of PU/Buckypaper composites sensor acted similarly to the conventional foil strain gage. Generally, both the sensor's and the strain gage's electric resistance change was essentially linear to the applied strain and was related to the gage factor (Figure 5.16). The first 4 minutes of the test indicated that the system had a residual strain to which the sensor and strain gage had to adapt to before the test was begun. The second loading occurred about 30 seconds after releasing the previous load. Again, a "no load" period between the $10^{\text {th }}$ and the $13^{\text {th }}$ minute was introduced 
before the loading was begun again. The strain gage appeared to have returned to "zero" but the PU/Buckypaper sensor appeared to lag and showed some hysteresis. The conventional foil strain sensor responded quickly according to the load applied to the beam. However, the PU/Buckypaper film sensor did not completely return to "zero".

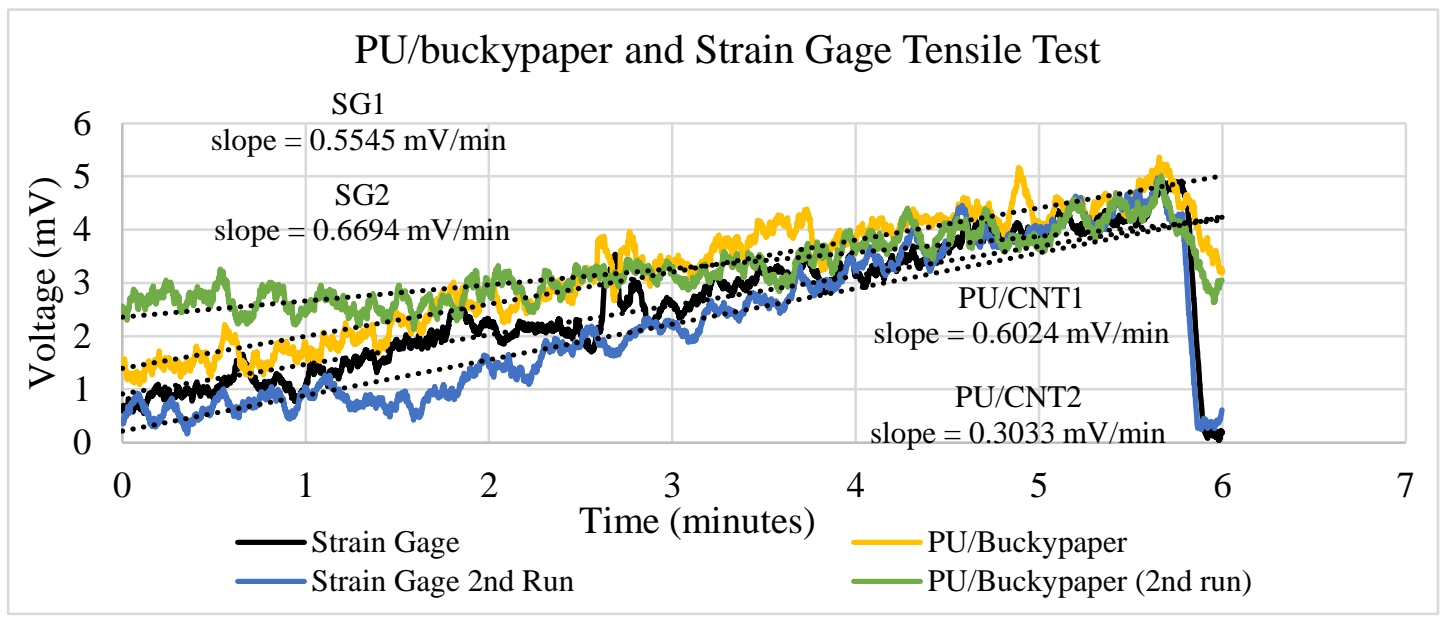

Figure 5.17 Linearity of repeat loading comparison of PU/Buckypaper and strain gage.

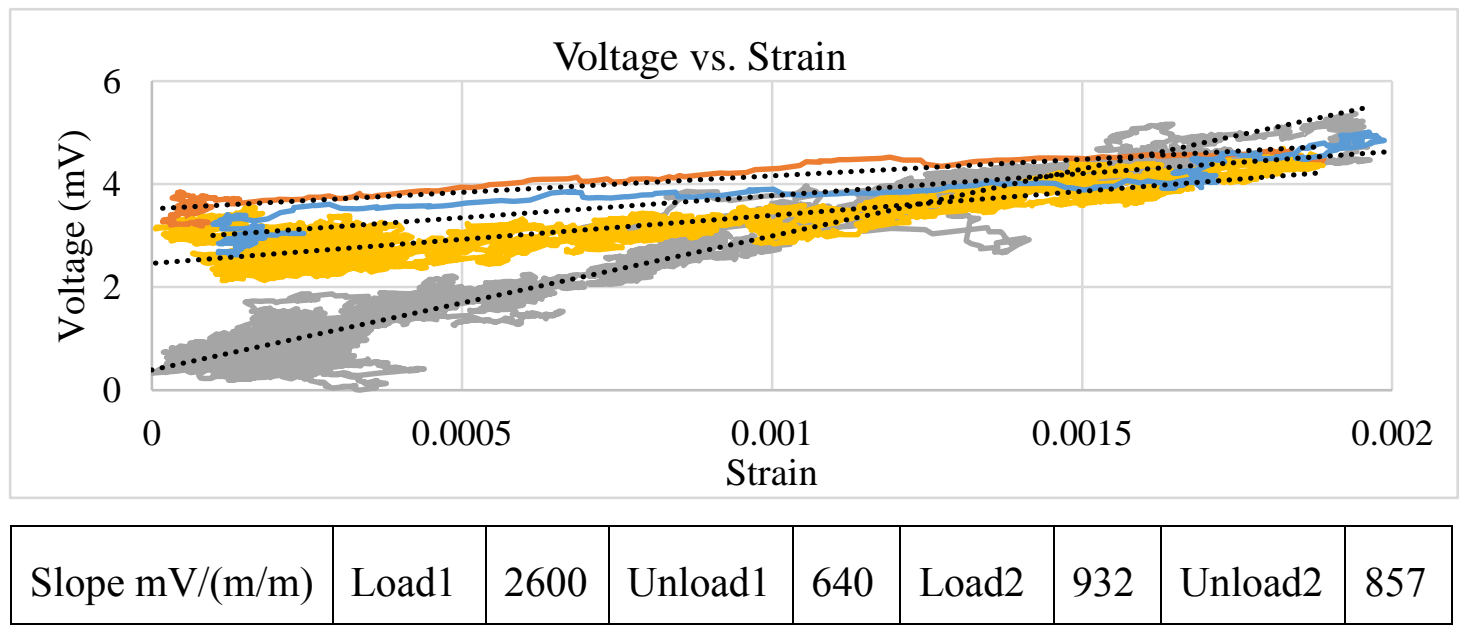

Figure 5.18 The loading/unloading comparison of PU/Buckypaper versus strain-first load (gray)/unload (red) and second load (yellow)/unload (blue) of the sensor.

As a new load was applied, thereby increasing the strain, the PU/Buckypaper composite sensor responded and sensed the strain in a linear way with the input. Yet, 
the slope of the response graph for the sensor appeared to be different than during the first load cycle. Hence, both sensors have a linear voltage output. By factoring out the lag in response of the second loading, all four output trend lines were deemed relatively parallel (Figures 5.17-5.18). Additionally, Figure 5.18 gives the voltage response relative to strain, as well, and the slopes of those lines for both types of sensors. Since the input voltage and output voltage were equivalent or relatively similar, so were the gage factors. Therefore, the PU/Buckypaper composite sensor was sensing similarly to the conventional foil strain gauge in terms of repeatability and linearity.

\subsubsection{Dynamic Sensing Characteristic}

Dynamic response performance is an important criterion for sensors. Standard foil strain gages were used for comparison. Herein, the frequency sweep method was used to study the sensitivity of the PU/Buckypaper and the foil strain gages to input frequencies. Frequency domain from $10 \mathrm{~Hz}$ to $1000 \mathrm{~Hz}$ was studied and the results are presented in Figure 5.17. The FFT graphs consistently showed a response at $60 \mathrm{~Hz}$ of $0.418 \mathrm{mV}$ for the PU/Buckypaper sensor and $1.47 \mathrm{mV}$ for the foil strain gage. This response was due to background source that was always present, namely the wall outlet source, and can be disregarded. The results displayed in Figures 5.17(a)-(n) were typical results for the sensor and strain gage.

Shown in Figures 5.19(a)-5.19(f) are the sensing results over the range of $10 \mathrm{~Hz}$ to $200 \mathrm{~Hz}$. In this range the PU/Buckypaper composite sensor was either the same or was superior to the normal foil strain gage for sensing purposes since the highest peak consistently occurred at the location of the input frequency. The foil strain gage was 
superior at $10 \mathrm{~Hz}$ and $200 \mathrm{~Hz}$ in the sense that it gave a significantly larger voltage response when compared to the composite sensor. In higher frequencies, the composite sensor either performed better or both signals were not very different from each other for the purposes of sensing.

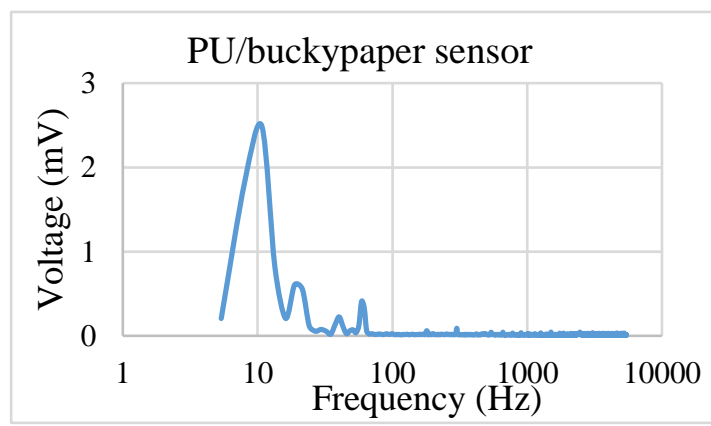

(a) $10 \mathrm{~Hz}$ - Magnitude: $2.4727 \mathrm{mV}$.

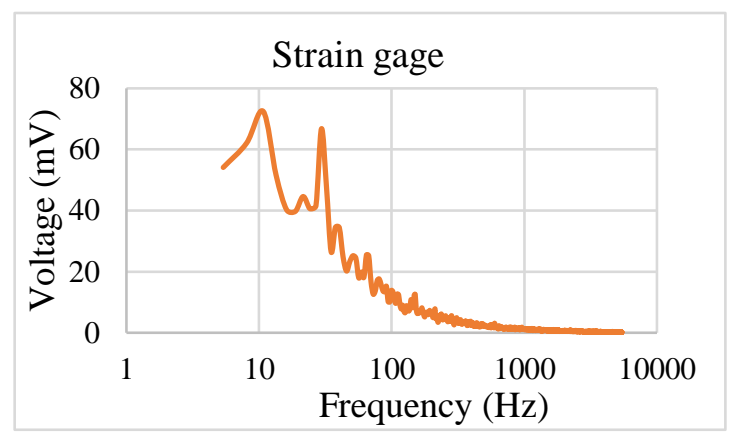

(b) $10 \mathrm{~Hz}$ - Magnitude: $70 \mathrm{mV}$.

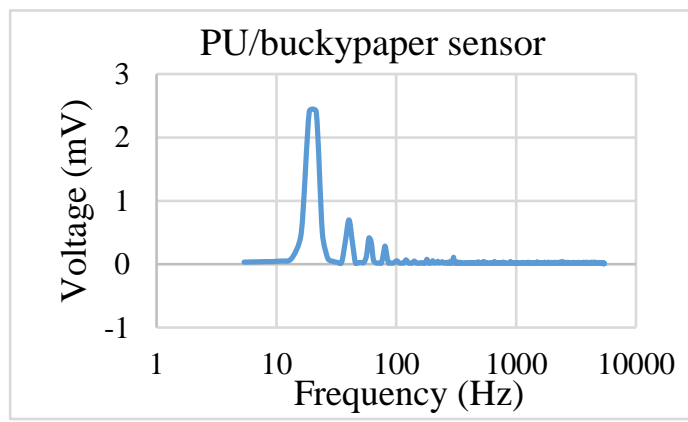

(c) $20 \mathrm{~Hz}$ - Magnitude: $2.4082 \mathrm{mV}$.

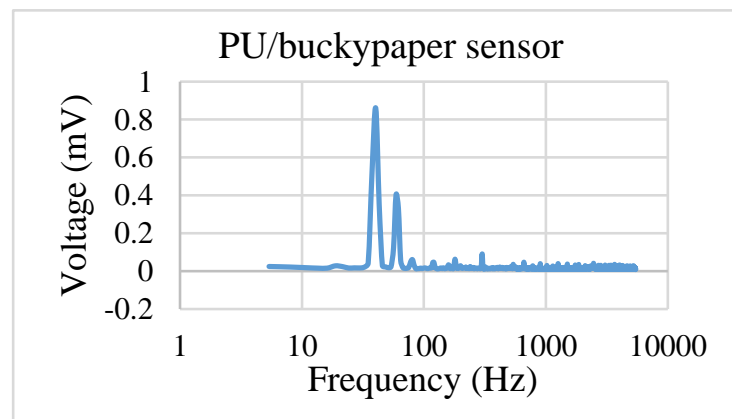

(e) $40 \mathrm{~Hz}$ - Magnitude: $0.8601 \mathrm{mV}$.

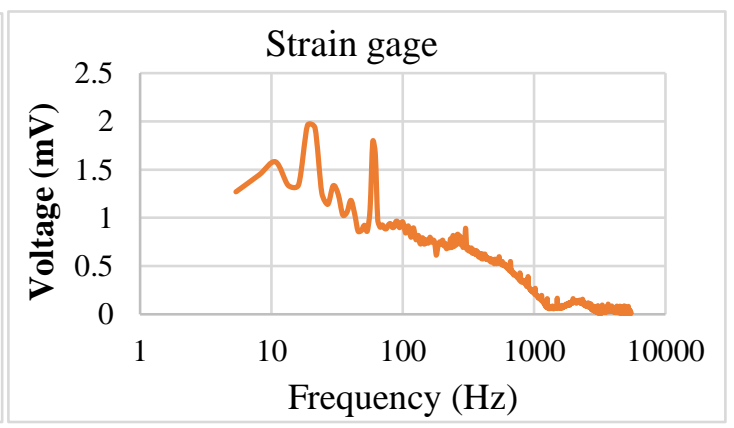

(d) $20 \mathrm{~Hz}$ - Magnitude: $1.9579 \mathrm{mV}$.

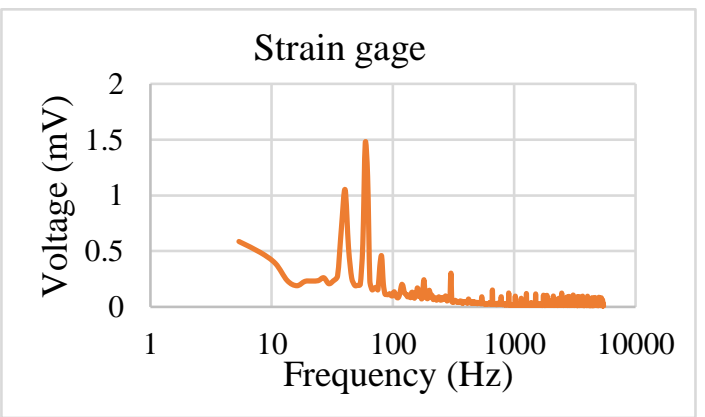

(f) $40 \mathrm{~Hz}$-Magnitude: $1.0515 \mathrm{mV}$.

Figure 5.19 Frequency response comparison of the strain gages and the PU/Buckypaper. 


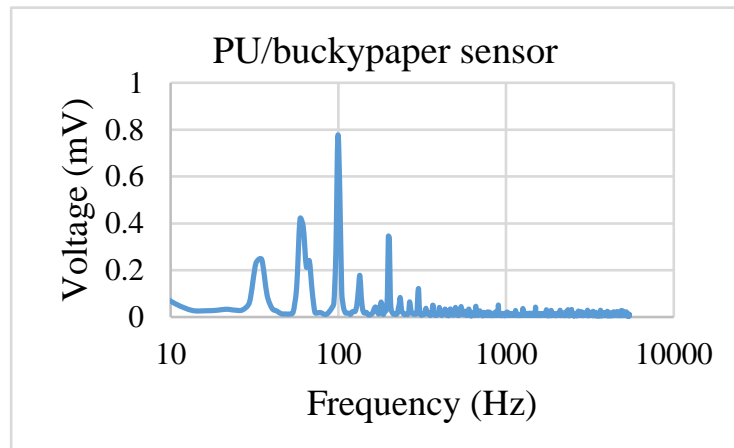

(a) $100 \mathrm{~Hz}$ - Magnitude: $0.7716 \mathrm{mV}$.

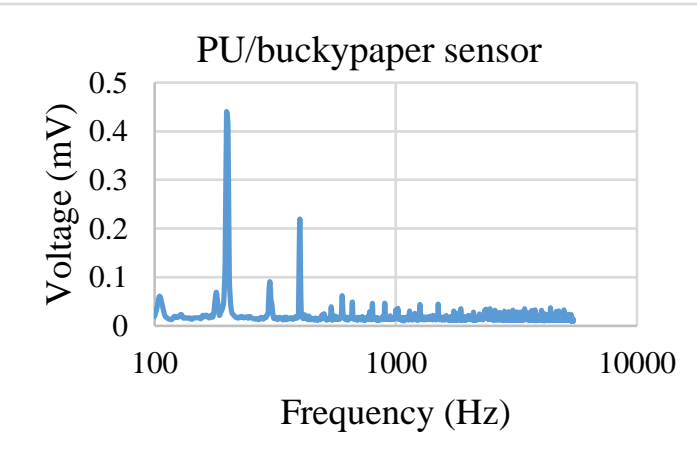

(c) $200 \mathrm{~Hz}$ - Magnitude: $0.4374 \mathrm{mV}$.

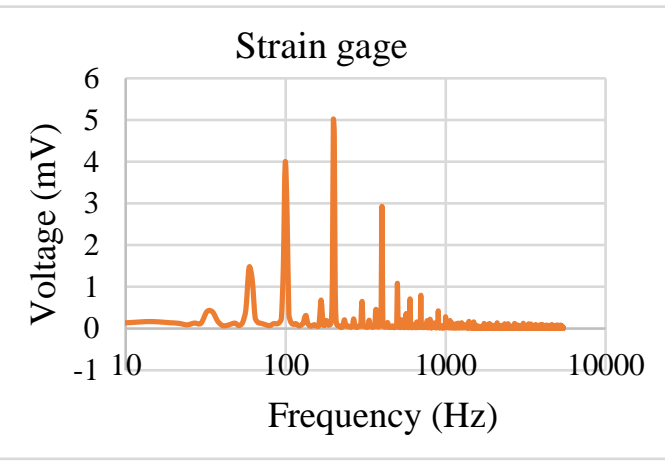

(b) $100 \mathrm{~Hz}$ - Magnitude: $3.9748 \mathrm{mV}$.

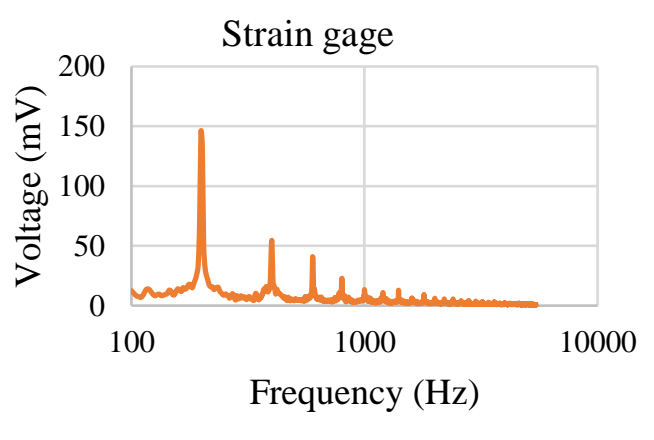

(d) $200 \mathrm{~Hz}$ - Magnitude: $145.43 \mathrm{mV}$.

Figure 5.20 Frequency response comparison of the strain gages and the PU/Buckypaper.

Figures 5.20 (a)-5.20 (d) indicates the frequency response in the frequency range 100-200 Hz. When the frequency was increased, the voltage output for both strain gage and PU/Buckypaper composites decreased significantly. The voltage output of PU/Buckypaper composites dropped to $0.77 \mathrm{mV}$ at a frequency of $100 \mathrm{~Hz}$ and the strain gage drops to about $3.98 \mathrm{mV}$. When the frequency was further increased to $1000 \mathrm{~Hz}$, the voltage output of both sensors remained of the same order, $0.0403 \mathrm{vs} .0 .0365 \mathrm{mV}$ (see Figures 5.21(a)-(d)). It means that the PU/Buckypaper composites strain sensors appeared to be better for structural health monitoring, which frequently uses the change of the natural frequency as an index for detecting small cracks. 


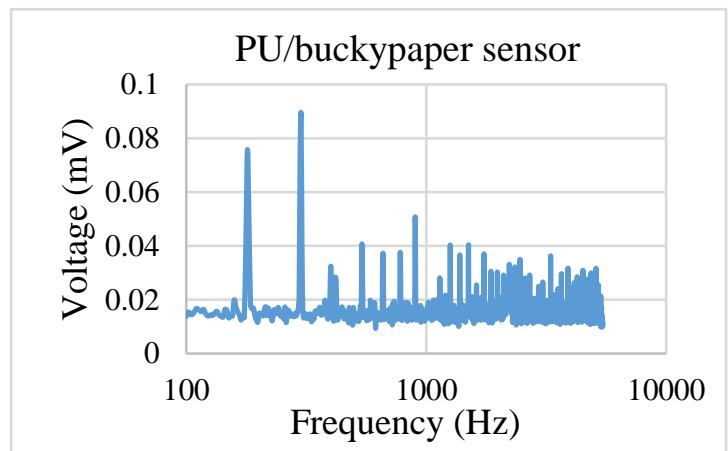

(a) $400 \mathrm{~Hz}$ - Magnitude: $0.0325 \mathrm{mV}$.

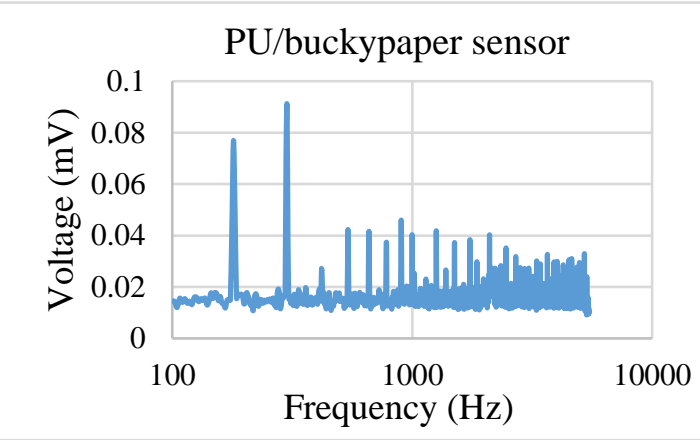

(c) $1000 \mathrm{~Hz}$ - Magnitude: $0.0403 \mathrm{mV}$.

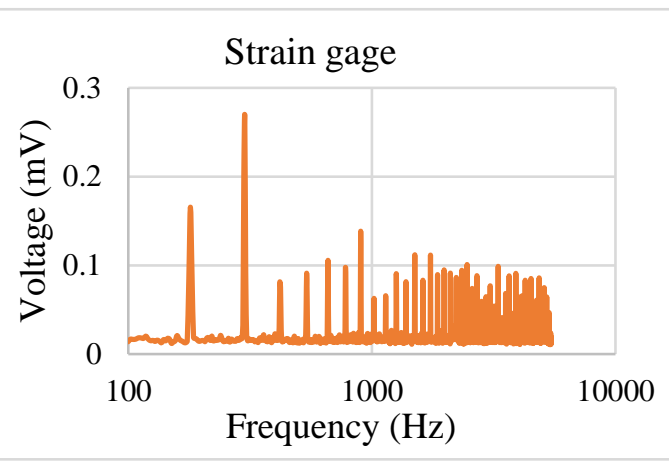

(b) $400 \mathrm{~Hz}$ - Magnitude: $0.0229 \mathrm{mV}$.

Figure 5.21 Frequency response comparison of the strain gages and the PU/Buckypaper.

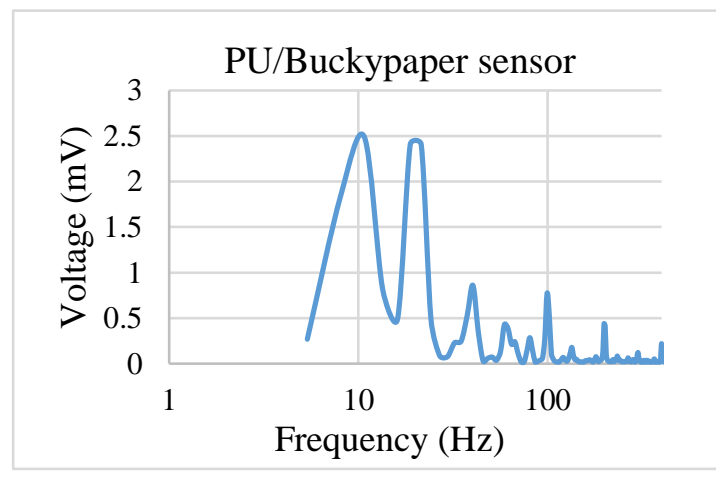

(a) FRF superposition - (10-400 Hz).

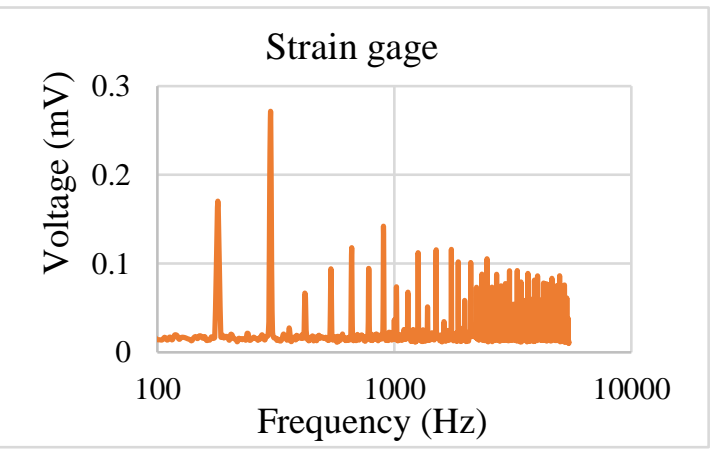

(d) $1000 \mathrm{~Hz}$ - Magnitude: $0.0365 \mathrm{mV}$.

Figure 5.22 Superposition of the frequency response functions of the PU/Buckypaper sensor and foil strain gage in the $10-400 \mathrm{~Hz}$ range.

Figures 5.22(a) and 5.22(b) provides the superposition of the FRFs shown in Figures 5.20-5.21 and represent the response of PU/Buckypaper or of the strain gage, 
respectively, to the different input frequencies. This is not a true FRF because the frequency sweep method must be used to generate these graphs. The graphs were drawn to provide a fuller picture of the dynamic sensing capabilities of each sensor. This is fund advantageous for the comparison of the sensor and strain gage because it showed how the signal could be used for frequency sensing. The graph for the PU/Buckypaper composite sensor showed a sensor that responded to frequencies in the range of 10 to $200 \mathrm{~Hz}$ while at the same time showing that each frequency signal was distinguishable from each other. The foil strain gage did not have this capability. The $10 \mathrm{~Hz}$ foil strain gage response was found to eclipse all the frequency responses up to $200 \mathrm{~Hz}$ and appeared to show a peak at $30 \mathrm{~Hz}$, a frequency for which no sampling was made. For the reasons stated, the PU/Buckypaper composite sensor appears to have responded in a superior manner when compared to a foil strain gage.

\subsection{Conclusion}

High aspect ratio $(>100)$ of $\mathrm{ZnO}$ nanowires were successfully synthesized. A PU/Buckypaper sensor was created to evaluate its sensing and damping capabilities versus a standard foil strain gage and constrained layer damping system. The free end vibration test results showed that for the single side attachment, the damping ratios for both PU film and Buckypaper were close, while PU/Buckypaper composites had higher damping ratios than their individual components. For example, with $63.6 \%$ coverage of PU/MWCNT on Al base beam, the damping ratio reached 0.194 , which was $35 \%$ to $56 \%$ higher than their individual components. For both single sided and double sided 
attachment, the damping ratios when clamping of the beam occurred on the sample side were much higher than when clamping of the beam occurred on the free end side.

The relationship between the loss factor and beam coverage, L1/L, was discussed for single and double sided attachment. Briefly, the loss factor, was found to increase with increasing coverage length, L1/L. The loss factor, then decreased before increasing again as the $\mathrm{L} 1 / \mathrm{L}$ continued to increase. The trend for double sided attachment was found to follow the trends discussed by Rao (2004) and Levy and Chen (1994).

The possibility of using the PU/Buckypaper as a strain sensor was also investigated. Though, there was a drift for the PU/Buckypaper sensor during the one-hour long drift test, the slope was relatively small. For example, for a one hour drift test, the voltage change for PU/MWCNT was $-0.6184 \mathrm{mV}$.

Two loading cycle tests showed that though there was residual strain for both the PU/Buckypaper sensor and the standard foil strain sensor for both loading cycles, the PU/Buckypaper sensor appeared to lag and showed some hysteresis in the second loading cycle. However, both sensors had a linear voltage output and all four output trend lines were deemed relatively linear, and, in the first cycle, also parallel. Therefore, the PU/Buckypaper composite sensor sensed in a similar fashion to the standard foil strain gauge in terms of repeatability and linearity.

The dynamic sensing test results showed that over the range of $10 \mathrm{~Hz}$ to $200 \mathrm{~Hz}$, the PU/Buckypaper composite sensor was comparable to or superior to foil strain sensor for sensing purposes since PU/MWCNT sensors showed more distinguished frequency response than foil strain gage. In higher frequencies, the composite sensor either 
performed better than the conventional foil strain gage or both signals in the sensor and the foil strain gage were not detectable to any accuracy for sensing- It means that the PU/Buckypaper sensor has the potential to be strain sensor.

Lastly, not all samples created were capable sensors. However, the ones that worked produced repeatable results. In comparison to viscoelastic material (VEM) constrained layer dampers discussed in (Chen and Levy, 1996; Levy and Chen, 1994), the same size PU/Buckypaper sensor would have a density that is $40 \%$ of the VEM and $6.4 \%$ of the density of the constraining layer, and would have similar damping behavior, but would have more useful attributes for a sensor. 


\section{CHAPTER VI}

\section{DISCUSSION OF YOUNG'S MODULUS}

Young's modulus, also known as the elastic modulus, is a measure of the stiffness of a solid material. It is a mechanical property of linear elastic solid materials. It defines the relationship between stress (force per unit area) and strain (proportional deformation) in a material (Roylance, 2008). For vibration and damping analysis of the structure with damping materials, complex-valued modulus containing elastic modulus and loss factor is frequently used by many researchers (e.g., Chen and Levy, 1996, 1998; Rao, 2004; Li et al, 2007), for it can fully consider the energy dissipation due to the damping materials.

There are several methods to test the Young's modulus of a materials. They include conventional tension method, pressure difference method, two-hydrophones method, vibration method and nanoindentation method. For each method, the deviations are different. For example, buckypaper has been demonstrated by nanoindentation method to have Young's modulus that can vary from $0.1 \mathrm{G}$ Pa to $12 \mathrm{G} \mathrm{Pa}$ (Li and Levy, 2007). This has happened because the random spaghetti-type arrangement of large numbers of MWCNT resulted in large local porosity and agglomerates. If the indenter happened to be applied in a very "solid" area with many aggregated MWCNT, the measured Young's modulus would be high, around $12 \mathrm{G} \mathrm{Pa}$. However, if the indenter happened to be applied in an "empty" location, the load relaxation then would take a long time and the measured Young's modulus would be a much smaller number.

The Nanoindentation method is useful for the atomic level characterization of 
Young's modulus. However, it requires strict smoothness of the tested sample. If the smoothness of the sample surface was not good, the deviation would be very high. Also for the two components composites, for example, for our samples, the results of nanoindentation of PANI/11\% MWCNT and PANI/ 22\% MWCNT would be scattered, which as shown in Figures 6.1 and 6.2, respectively.

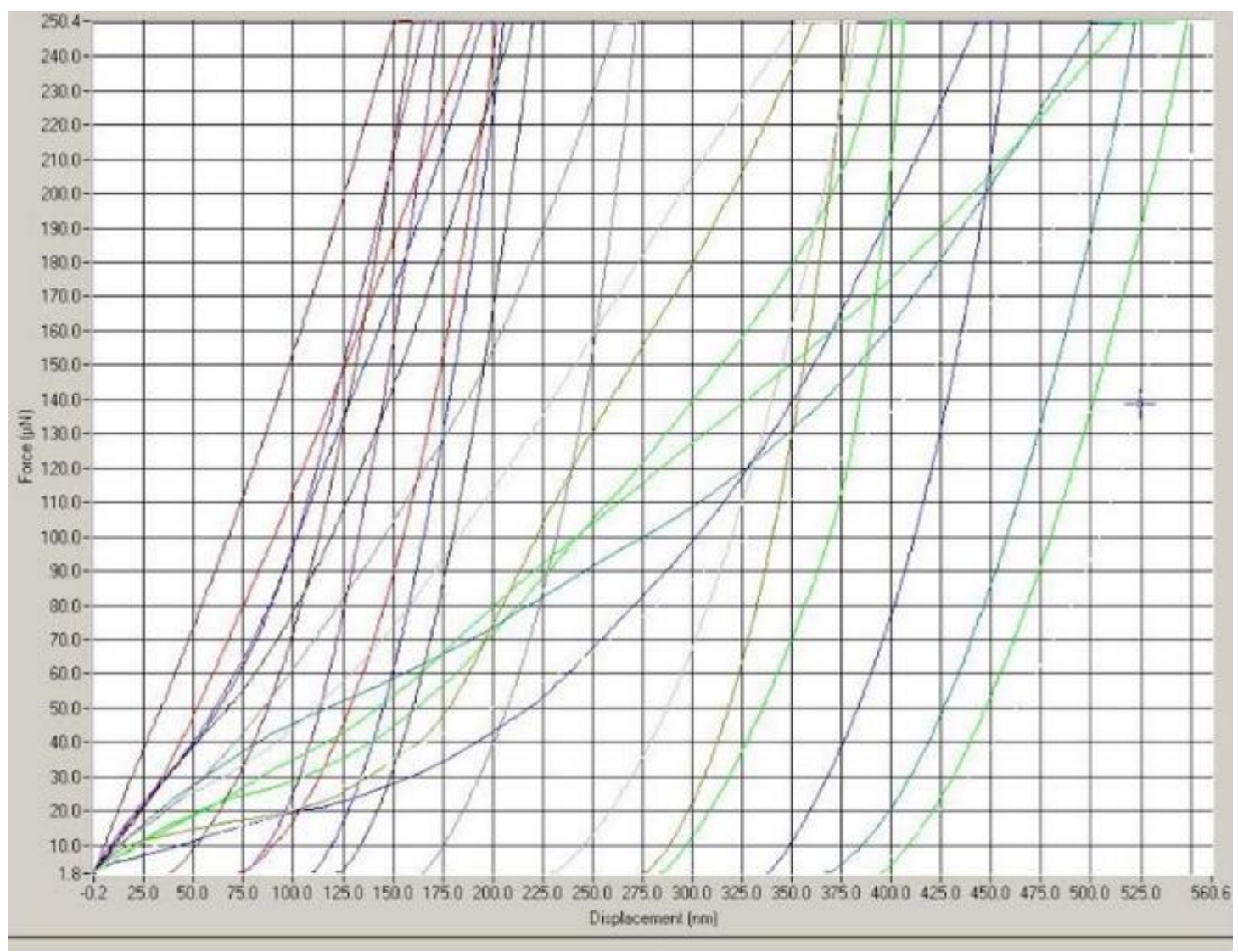

Figure 6.1 Nanoindentation (loading-unloading curves) of PANI/11\% MWCNT sensor.

Their Young's modulus from these tests were found to be $2.72 \pm 1.54 \mathrm{G} \mathrm{Pa}$ and 2.37 $\pm 0.9125 \mathrm{G} \mathrm{Pa}$. There were several reasons for this phenomenon. Two of the most important ones were the non-smoothness of the samples' surface and the different components of the composites. As was found in Chapter III, the surface smoothness of 
PANI/MWCNT was between their individual components and PANI was found to have the smoothest surface of both pure MWCNT and PANI/MWCNT composite for the same fabrication method. The other reason was the different proportion of their components. MWCNT has been demonstrated to have Young's modulus of 0.91 1.24 T Pa (Qia, 2003), which is much higher than the PANI whose Young's modulus was reported as 1.3 $\pm 0.2 \mathrm{G} \mathrm{Pa}$ (Valentová, 2010).

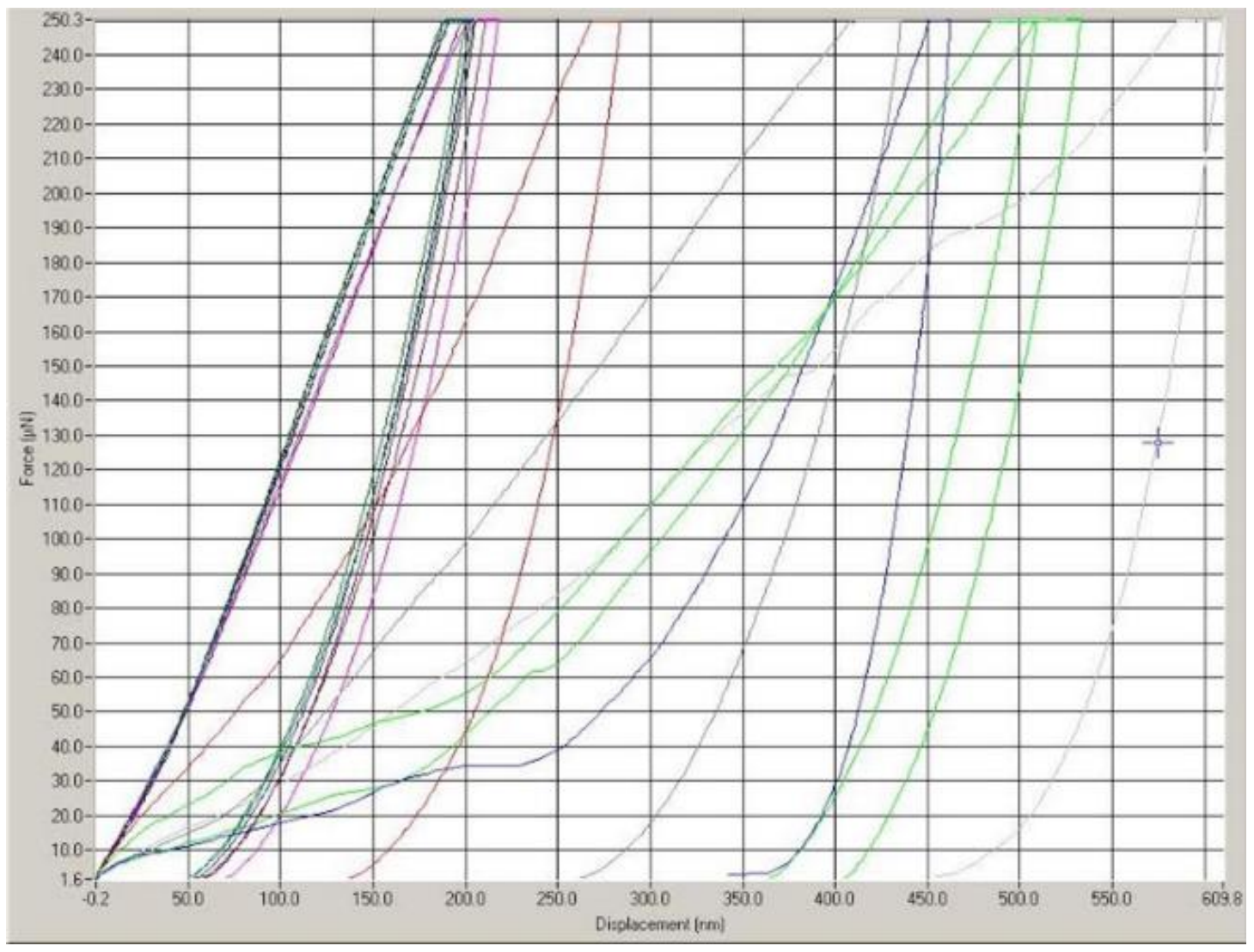

Figure 6.2 Nanoindentation (loading-unloading curves) of PANI/22\% MWCNT sensor.

Figure 6.3 was the result of the MTS tension test and shows the load-displacement relationship of the PANI/11\% MWCNT. Three loading cycles were applied to the same sample. From the Figure 6.3, the first and second line are almost parallel to each other. 
However, for the third run, the sample broke and the grips moved away from each other showing more displacement when no load was applied. This was reasonable, since our sample is a polymer composite and its internal structure will be adjusted once the strain was applied, especially the first time the strain was applied, which was also demonstrated in Chapter IV.

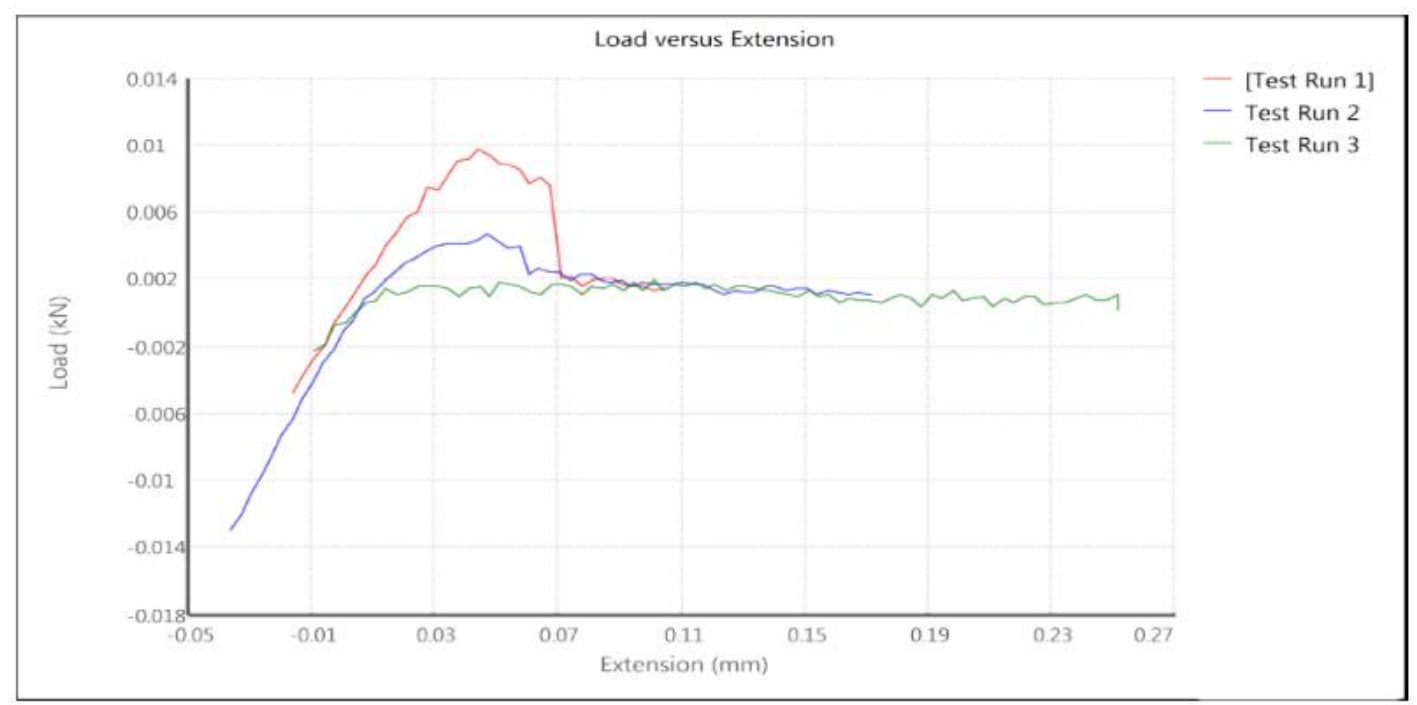

Figure 6.3 Tension test method (load-displacement) result for PANI/11\% MWCNT.

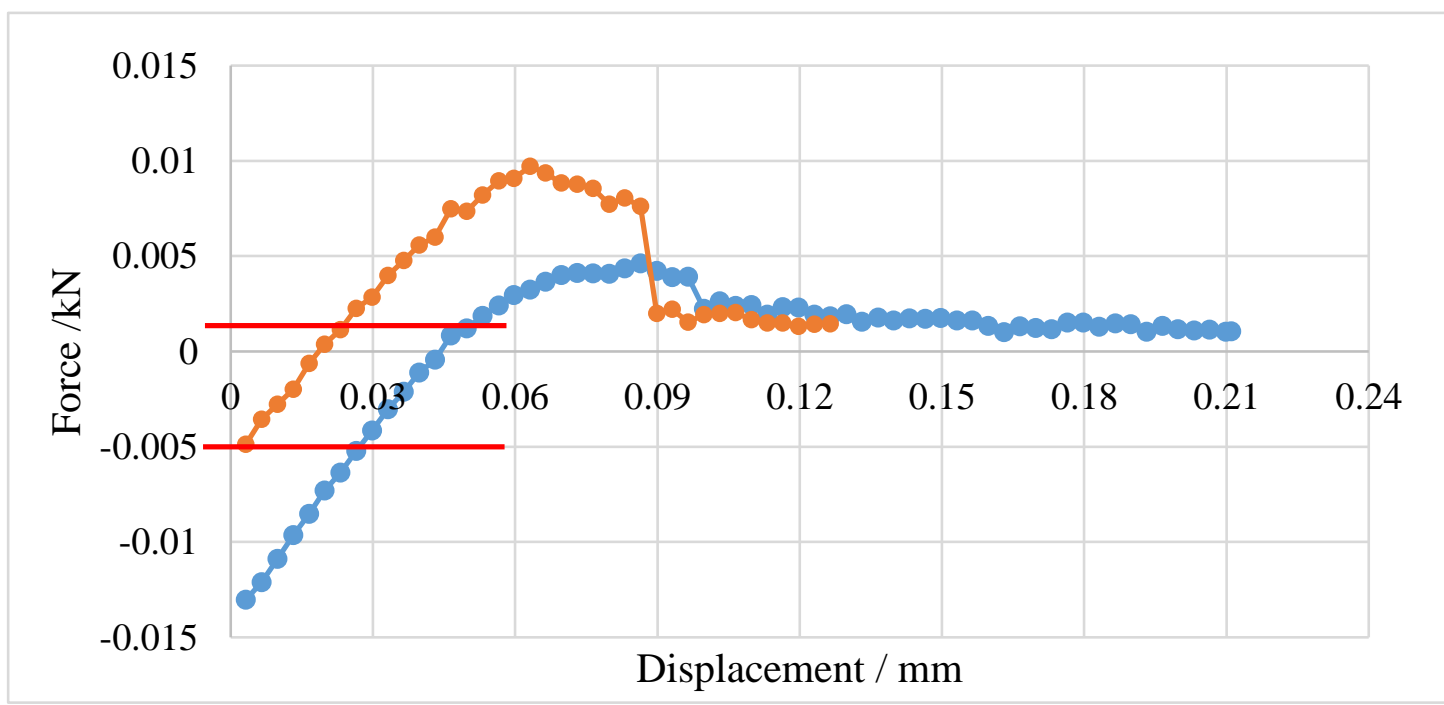

Figure 6.4 Tension test $\left(1^{\text {st }}\right.$ and $2^{\text {nd }}$ load-displacement) result for PANI/11\% MWCNT. 
From the $1^{\text {st }}$ and $2^{\text {nd }}$ load and displacement curves data was extracted and is shown in Figure 6.4. The Young's Modulus was calculated over the common set of loading points shown in red. The Young's modulus was then calculated using a linear least squares method and values of $0.1833 \mathrm{G} \mathrm{Pa}$ and $0.1864 \mathrm{G}$ Pa were obtained, respectively, showing a difference of only $1.6 \%$. The linear least square method correlation coefficient for both lines were also compared and were found to be 0.9970 and 0.9947 , respectively, which meant that the linear least squares method had excellent fidelity to the data.

However, the values of Young's modulus of PANI/11\% MWCNT tested by nanoindentation and tension methods were very different because the MTS test measured the global value of the Young's Modulus while the nanoindentation test measured the local value and the two results are found to be very different. 


\section{CHAPTER VII \\ CONCLUSION AND FUTURE WORK}

In this dissertation, Polyaniline/Multi-walled carbon nanotube film composites and PU/Buckypaper film composites were prepared and their structural damping properties and strain sensing properties were mainly investigated. Based on these experimental results, frit compression method made PANI/MWCNT composites films and layer by layer method made PU/MWCNT films show that they have structural damping properties simultaneously with strain sensing properties. The main conclusions are summarized here.

\subsection{The Multifunctional PANI/MWCNT Composites Films}

Both circular and rectangular shapes of PANI/MWCNT composites films were made by frit compression method. Different compositions of PANI/MWCNT nanocomposites films were compared by testing their structural damping properties and strain sensing properties. Free vibration tests result showed that PANI/11\% MWCNT displayed higher damping ratios than pure PANI, PANI/22\% MWCNT, PANI/44\% MWCNT and pure MWCNT.

The location of the clamped support of the cantilever beam was very important to the magnitude of the damping ratio while performing the free vibration damping tests. When the beam was clamped close to the sample end, with the sample between the clamp and the free end, it displayed higher damping ratios than when the beam was clamped to the free, uncovered end. The results showed that by attaching the 
nanocomposite sample to both sides of $\mathrm{Al}$ beam, the damping ratio was much higher than for the single sided coverage. By attaching the nanocomposite sample to both sides of $\mathrm{Al}$ beam, the damping ratio reached a value of 0.072 , which is 18.85 times higher than for the single sided coverage. The relationship of loss factor, $\eta$, and beam coverage length, L1/L, was discussed for single sided and double sided attachment. The loss factor, $\eta$, increases as coverage length, L1/L, increases. The loss factor, $\eta$, then decreases before increasing again as the coverage length, L1/L, continues to increase.

The strain sensor result showed that pure PANI, PANI/11\% MWCNT, PANI/22\% MWCNT and PANI/44\% MWCNT showed some stability like traditional foil strain gauge. The PANI/MWCNT sensor's relation between load and displacement or strain is not described by a single equation as found for a strain gage. It appears to have three regions in which the relationship is quasi-linear. This is in line with an observation reported elsewhere (Levy, 2015), namely, adding too much PANI to the sensor patch destroys the linear sensing capability of the sensor patch.

The dynamic sensing test results showed that over the range of $10-1000 \mathrm{~Hz}$, the PANI/11\% MWCNT composite sensor was consistently superior to foil strain gage for sensing purposes since the highest peak consistently corresponded to the input frequency and was much higher, for example, at $20 \mathrm{~Hz}, 820$ times higher than those of the strain gage. In higher frequencies, the composite sensor performed better but the signal peaks were not the largest. Drift test of the sensor showed that it was stable. It showed that the PANI/MWCNT sensor was stable over many hours changing by 0.25 $\mathrm{mV}$ only over a $20 \mathrm{~h}$ period. 


\subsection{The Multifunctional PU/Buckypaper Films}

PU/Buckypaper films were made by pouring liquid PU upon Buckypaper. Free end vibration test results show that PU/Buckypaper composites have higher damping ratios than their individual components. The relationship of loss factor, and beam coverage L1/L was discussed for single sided and double sided attachment. Briefly, the loss factor, increases with coverage length $\mathrm{L} 1 / \mathrm{L}$ increases. The loss factor, then decreases before increasing again as the coverage length $\mathrm{L} 1 / \mathrm{L}$ continues to increase.

For the strain sensor test, though, there is a drift for the PU/Buckypaper sensor during the one-hour long drift test, the slope is relatively small. And the PU/Buckypaper composite sensor senses similarly to the standard foil strain gauge in terms of repeatability and linearity. The dynamic sensing test results showed that over the range of $10 \mathrm{~Hz}$ to $200 \mathrm{~Hz}$, the PU/Buckypaper composite sensor was comparable to or superior to foil strain sensor for sensing purposes since PU/MWCNT sensors showed more distinguished frequency response than foil strain gage. In higher frequencies, the composite sensor either performed better or both signals were not detectable to any accuracy for sensing than the conventional foil strain gage. In higher frequencies, the composite sensor either performed better or both signals were not detectable to any accuracy for sensing.

\subsection{PU/ZnO/MWCNT composites}

$\mathrm{ZnO}$ nanowires with high aspect ratio large than 100 were successfully synthesized. However, it's difficulty to get good dispersion of MWCNT and ZnO nanowires/ 
nanorods into the PU matrix, which lead to the value of the electrical value of the $\mathrm{PU} / \mathrm{MWCNT} / \mathrm{ZnO}$ nanowires/nanorods composites films too high to be strain sensors.

\subsection{Future Research}

The following topics are potential research areas of interest in the future:

(a) Discussion of the partially covered PANI/MWCNT multifunctional materials. As we already did a lot of experiments in this area, there is a need to define the theoretical support for the partially covered PANI/MWCNT multifunctional materials. In this manner, a further analysis of the data can be accomplished when evaluating changes in parameters.

(b) Investigation of the combination of CNT, Zinc Oxide nanorod and polymer as strain sensors and structural damping materials and their preparation methods. The Zinc Oxide nanorod has good piezeoelectrical properties. However, the problem is how to create this type of composite and how to achieve good dispersion of $\mathrm{ZnO}$ and MWCNT into the polymer matrix.

(c) Study of the environmental effects, such as temperature, humidity, fast cooling and heating, on the multifunctional materials. As has been discussed by Li et al. (2007), environmental effects can play an important role in the mechanical characterization of the composite.

(d) Mathematical modeling for the MWCNT composites by either molecular dynamics methods or other methods to explore its electrical and mechanical properties, which will further our understanding of multifunctional materials for both damping and strain sensing properties. 


\section{References}

Agrawal, R., Nieto, A., Chen, H., Mora, M. and Agarwal, A., 2013, Nanoscale damping characteristics of boron nitride nanotubes and carbon nanotubes reinforced polymer composites. ACS Applied Materials \& Interfaces, 5 (22): 12052-12057.

Alamusi, W., Yuan, F., Surina, Y., Li, Hu, N., Ning, H. M., Liu, Y. L., Wu, L. K., Atobe, S. and Fukunaga, H., 2013, Ultrasensitive strain sensors of multiwalled carbon nanotube/epoxy nanocomposite using dielectric loss tangent. Applied Physics Letters, 103: 221903/1-221903/5.

Almkhelfe, H., Carpena-Nunez, J., Back, T. C. and Amama, P. B., 2016, Gaseous product mixture from Fischer-Tropsch synthesis as an efficient carbon feedstock for low temperature CVD growth of carbon nanotube carpets. Nanoscale, 8:13476-13487.

Alnefaie, K. A., Aldousari, S. M. and Khashaba, U. A., 2013, New development of selfdamping MWCNT composites: Composites Part A: Applied Science and Manufacturing, 52:1-11.

Alva, A. and Raja, S., 2014, Damping characteristics of epoxy-reinforced composite with multiwall carbon nanotubes: Mechanics of Advanced Materials and Structures, 21(3): 197-206.

Ansari, R., Faghih, M., Shojaei, V., Mohammadi, Gholami, R. and Sadeghi, F., 2014, Nonlinear forced vibration analysis of functionally graded carbon nanotubereinforced composite Timoshenko beams: Composite Structures, 113: 316-327.

Anton, S. R., Erturk A. and Inman, D. J., 2010, Multifunctional self-charging structures using piezoceramics and thin-film batteries. Smart Materials and Structures, 19 (11): 115021/1-115021/15.

Balani, K., Bakshi, S. R., Chen, Y., Laha, T. and Agarwal, A., 2007, Role of powder treatment and carbon nanotube dispersion in the fracture toughening of plasmasprayed aluminum oxide-carbon nanotube nanocomposite. Journal of Nanoscience and Nanotechnology, 7: 3553-3562.

Bandarian, M., Shojaei, A. and Rashidi, A. M., 2011, Thermal, mechanical and acoustic damping properties of flexible open-cell polyurethane/multi-walled carbon nanotube foams: effect of surface functionality of nanotubes. Polymer International, 60: 475-482.

Bayer, O., 1947, Das Di-Isocyanat-Polyadditionsverfahren (Polyurethane). Angewandte Chemie, 59: 257-272. 
Boz, U., and Basdogan, I., 2015, IIR filtering based adaptive active vibration control methodology with online secondary path modeling using PZT actuators. Smart Materials and Structures, 24: 125001/1-125001/12.

Buffa, F., Abraham, G. A., Grady, B. P. and Resasco, D., 2007, Effect of nanotube functionalization on the properties of single-walled carbon nanotube/polyurethane composites. Journal of Polymer Science, Part B: Polymer Physics, 45: 490-501.

Carmona, F., Canet, R. and Delhaes, P., 1987, Piezoresistivity of heterogeneous solids. Journal of Applied Physics, 61(7): 2550-2557.

Carmona, F. and Amarti, A.E., 2016, Temperature and pressure dependence of the conductivity anisotropy of unidirectional short carbon fiber filled polymers. Journal of Materials Research, 7(1): 117-123.

Chang, F.Y., Wang, R.H., Yang, H., Lin, Y.H., Chen, T.M. and Huang, S.J., 2010, Flexible strain sensors fabricated with carbon nano-tube and carbon nano-fiber composite thin films. Thin Solid Films, 518: 7343-7347.

Chang L., 2005, Foundations of MEMS: Upper Saddle River, NJ 07458, Pearson Prentice Hall, 22 p.

Chen, M., Chen, C.D. and Yang, Y.S., 2001, Torsion of the previously normal uterine adnexa. Acta Obstetricia et Gynecologica Scandinavica, 80 (1): 58-61.

Chen, Q. and Levy, C., 1996, Vibration characteristics of partially covered doublesandwich cantilever beam. AIAA Journal, 34 (12): 2622-2626.

Chen, Q. and Levy, C., 1998, Comparison of theory with experimental data for a partially covered double-sandwich cantilever beam. International Journal of Mechanical Sciences, 40(4): 399-403.

Chen, Y.R. and Weng, C.I., 2007, Electronic properties of zigzag carbon nanotubes under uniaxial strain. Carbon, 45(8): 1636-1644.

Costa, P., Silvia, C., Viana, J. C. and Lanceros Mendez, S., 2014, Extruded thermoplastic elastomers styrene-butadiene-styrene/carbon nanotubes composites for strain sensor applications. Composites, Part B: Engineering, 57: $242-249$.

Crosby, H. L., 1980, Centrifuge with torsional vibration sensing and signaling. pp.18.

Dharap, P., Li, Z., Nagarajaiah, S. and Barrera, E. V., 2004, Nanotube film based on single-wall carbon nanotubes for strain sensing. Nanotechnology,15(3):379-382. 
El-Raheb, M. and Wagner, P., 1986, Damped Response of Shells by a Constrained Viscoelastic Layer. Journal of Applied Mechanics, 53 (4): 902-908.

Fang, C., Lei, W., Zhou, X., An, N. and Li, J., 2015, Heat-resistant high-damping polyurethane elastomer and preparation method thereof. pp. 13

Fazita, N. M. R., Jayaraman, K., Bhattacharyya, D., Hossain, S. M., Haafiz, M. M. K. and H.P.S., K. A., 2015, Disposal options of bamboo fabric-reinforced poly(Lactic) acid composites for sustainable packaging: biodegradability and recyclability. Polymers, 7(8): 1476-1496.

Feng J., 2009, Method for preparing superhydrophobic surface by using nano-particles for assisting micromolding. Chinese Patent: CN101537682A.

Feng, J., Zhong, M. and Lin, W., 2012, Fabrication of polyethylene surface with stable superhydrophobicity by nanoparticle assisted thermal micromolding process. Journal of Nanoscience and Nanotechnology, 12 (3): 2679-2684.

Ferry, J. D., 1970, Viscoelastic Properties of Polymers. 2nd edition. pp.671.

Finegan, I. C., Tibbetts, G. G. and Gibson, R. F., 2003, Modeling and characterization of damping in carbon nanofiber/polypropylene composites. Modeling and Characterization of Nanostructured Materials, 63 (11): 1629-1635.

Frogley, M. D., Zhao, Q. and Wagner, H. D., 2002, Polarized resonance Raman spectroscopy of single-wall carbon nanotubes within a polymer under strain. Phys. Rev. B, 65(11): 113413.

Gong, Z., Yan, X., Gong, J., Wang, J. and Wang, B., 2012, Investigation of free volume and damping property for polycarbonate/multiwalled carbon nanotube composites by positron annihilation technology. Journal of Applied Polymer Science, 125: 4028-4033.

Hazarika, A. and Maji, T. K., 2014, Strain sensing behavior and dynamic mechanical properties of carbon nanotubes/nanoclay reinforced wood polymer nanocomposite. Chemical Engineering Journal, 247: 33-41.

He, J., Zhong, F., Gao, X., Zhu, J., Sun, S. and Li, W., 2008, Damping materials composed of polyurethane, piezoelectric ceramic powder and polyaniline coating ceramic. Cailiao Kexue Yu Gongyi, 16: 506-510.

He, X., Liu, G., Yan, B., Suo, H. and Zhao, C., 2016, Significant enhancement of electrochemical behaviour by incorporation of carboxyl group functionalized carbon nanotubes into polyaniline based supercapacitor. European Polymer Journal, 83: 53-59. 
Hong, S. M., Lee, H. J., Han, T. H., Han, K. B. and Choi, Y., 2012, Development of strain sensor using carbon nanotubes film. IACSIT International Journal of Engineering and Technology, 4: 413-415.

Hong, S. Y., Varadan, V. V. and Varadan, V. K., 1992, Experiments on active vibration control of a thin plate using disk type piezoceramic actuators and sensors. Act. Mater. Adapt. Struct., 707-711.

Hu, N., Fukunaga, H., Lu, C., Kameyama, M. and Yan, B., 2005, Prediction of elastic properties of carbon nanotube reinforced composites. Proceedings of the Royal Society A: Mathematical, Physical and Engineering Science, 461(2058):16851710 .

Hu, N., Nunoya, K., Pan, D., Okabe, T. and Fukunaga, H., 2007, Prediction of buckling characteristics of carbon nanotubes. International Journal of Solids and Structures, 44 (20): 6535-6550.

Hu, N., Karube, Y., Yan, C., Masuda, Z. and Fukunaga, H., 2008, Tunneling effect in a polymer/carbon nanotube nanocomposite strain sensor. Acta Materialia, 56 (13): 2929-2936.

Hu, N., Li, Y., Nakamura, T., Katsumata, T., Koshikawa, T. and Arai, M., 2012, Reinforcement effects of MWCNT and VGCF in bulk composites and interlayer of CFRP laminates. Nanomechanics and Nanocomposites: Mechanical Behaviors, 43 (1): 3-9.

Huang, F., Vanhaecke, E. and Chen, D., 2010, In situ polymerization and characterizations of polyaniline on MWCNT powders and aligned MWCNT films. Catalysis Today, 150: 71-76.

Iijima, S., 1991, Helical microtubules of graphitic carbon: Nature, 354 (6348): 56-58.

Ikhouane, F. and Dyke, S. J., 2007, Modeling and identification of a shear mode magnetorheological damper. Smart Materials and Structures, 16 (3): 605.

Ishida, H. and Allen, D. J., 1996, Physical and mechanical characterization of near-zero shrinkage polybenzoxazines. Journal of Polymer Science Part B-polymer Physics, 34 (6): 1019-1030.

Jing, X., Geng, D., Chai, K., Wang, S. and Dong, W., 2015, Method for preparation of polyurethane elastomer vibration damper plate used in high-speed railway. Chinese Patent: 104877103 A 20150902.

Johnson, C. D., 1995, Design of passive damping systems. Journal of Vibration and Acoustics, 117:171-176. 
Kang, I., Kim, M., Shanov, V. and Shi, D., 2006, A carbon nanotube strain sensor for structural health monitoring. Smart Materials and Structures, 15 (3): 737-748.

Kawabe, H., Tsukiyama, N. and Yoshida, K., 2006, Active vibration damping based on neural network theory. Materials Science \& Engineering, A: Structural Materials: Properties, Microstructure and Processing, A442 (1): 547-550.

Kehlenbach, M., 2006, Actuator and sensor system for composite structures. US Patent: US7017421B2.

Kerwin, E. M., 1959, Damping of flexural waves by a constrained viscoelastic layer. The Journal of the Acoustical Society of America, 31 (7): 952-962.

Kim, J. W., Choi, H. J., Lee, H. G. and Choi, S. B., 2001, Damping characteristics of polyaniline-based electrorheological fluid. Journal of Industrial and Engineering Chemistry, 7: 218-222.

Kim, S. J., Kim, J. H. and Park, J. H., 2011, Measurement of characteristics of PU/MWNT films made of modified MWNT by dry heat treatment. Journal of Fiber Bioengineering and Informatics, 3 (4): 216-223.

Hu, N., Nunoya, K., Pan, D., Okabe, T. and Fukunaga, H., 2007, Prediction of buckling characteristics of carbon nanotubes. International Journal of Solids and Structures, 44 (20): 6535-6550.

Kireitseu, M., 2006, Vibration damping/dynamic properties of CNT-reinforced composite structures. NSTI Nanotechnol. Conf. Trade Show: Nano Science and Technology Institute, pp. 198-201.

Kogelnik, H. J., Adam, N., Prolingheuer, E. C. and Henrichs, P., 1992, Microcellular polyurethane elastomers as damping components in various applications. American Society of Mechanical Engineers, 38: 41-54.

Koratkar, N., Wei, B. and Ajayan, P. M., 2002, Carbon nanotube films for damping applications. Advanced Materials, 14: 997-1000.

Lall, A. K., Asnani, N. T. and Nakra, B. C., 1987, Vibration and damping analysis of rectangular plate with partially covered constrained viscoelastic layer. Journal of Vibration, Acoustics, Stress, and Reliability in Design, 109 (3): 241-247.

Levy, C., 2014, Interim progress report for proposal: no. 58940-EG-REP, Army Research Office.

Levy, C. and Chen, Q., 1994, Vibration analysis of a partially covered, double sandwich-type, cantilever beam. Journal of Sound and Vibration, 177(1): 1529. 
Li, X., and Levy, C., 2007a, Multi-walled carbon nanotube film as strain sensors for structural vibration control. in NSTI Nanotech 2007, Nanotechnol. Conf. Trade Show: CRC Press, p. 256-259.

Li, X., and Levy, C., 2007b, Multi-walled carbon nanotube film as strain sensors for structural vibration control. in NSTI Nanotech 2007, Nanotechnol. Conf. Trade Show: CRC Press, p. 256-259.

Li, X. and Levy, C., 2009, A novel strain gauge with damping capability. Sensors \& Transducers Journal, 7: 5-14.

Li, X., Levy, C. and Elaadil, L., 2008, Multiwalled carbon nanotube film for strain sensing. Nanotechnology, 19: 045501/1-045501/7.

Li, X., Wei, J., Chai, Y., Zhang, S. and Zhou, M., 2015, Different polyaniline/carbon nanotube composites as Pt catalyst supports for methanol electro-oxidation. Journal of Materials Science, 50(3):1159-1168.

Li, Y., Hori, N., Arai, M., Hu, N., Liu, Y. and Fukunaga, H., 2009, Improvement of interlaminar mechanical properties of CFRP laminates using VGCF. Special Issue: CompTest 2008, 40 (12): 2004-2012.

Li, Y., Hu, N., Yamamoto, G., Wang, Z., Hashida, T., Asanuma, H., Dong, C., Okabe, T., Arai, M. and Fukunaga, H., 2010, Molecular mechanics simulation of the sliding behavior between nested walls in a multi-walled carbon nanotube. Carbon, 48 (10): 2934-2940.

Li, Y., Liu, Y., Peng, X., Yan, C., Liu, S. and Hu, N., 2011, Pull-out simulations on interfacial properties of carbon nanotube-reinforced polymer nanocomposites. Computational Materials Science, 50 (6): 1854-1860.

Li, Y., Hu, K., Jiao, H., Liu, X., Wang, Q., Pan, G., Zhang, X. and Wang, T., 2015, In situ polymerization, thermal, damping, and mechanical properties of multiwalled carbon nanotubes/polyisobutylene-based polyurethane nanocomposites. Polymer Composites, 36 (1): 198-203.

Li, Y., Jiao, H., Pan, G., Wang, Q. and Wang, T., 2016, Mechanical and damping properties of carbon nanotube-modified polyisobutylene-based polyurethane composites. Journal of Composite Materials, 50: 929-936.

Li, Z., Dharap, P., Nagarajaiah, S., Barrera, E. V. and Kim, J. D., 2004, Carbon nanotube film sensors. Advanced Materials, 16 (7): 640-643.

Lin W., Rotenberg, Y., Fekrmandi, H., Ward, K. P. and Levy, C., 2015, Multifunctional materials of polyurethane/buckypaper for structural vibration damping and strain sensing. IJIRSET, 4 (12): 12531-12544. 
Lin, W., Rotenberg, Y., Ward, K., Fekrmandi, H. and Levy, C., 2016, Polyaniline/MultiWalled Carbon Nanotube Composites for Structural Vibration Damping and Strain Sensing. Journal of Materials Research, 1-11.

Liu, A., Wang, K. W. and Bakis, C. E., 2010, Multiscale damping model for polymeric composites containing carbon nanotube ropes. Journal of Composite Materials, 44: $2301-2323$.

Liu, J., Engquist, I. and Berggren, M., 2013, Double-gate light-emitting electrochemical transistor: confining the organic $\mathrm{p}-\mathrm{n}$ junction. Journal of the American Chemical Society, 135(33): 12224-12227.

Liu, L., Pan, C., Zhang, L. and Guo, B., 2016, A novel and non-cytotoxic self-healing supramolecular elastomer synthesized with small molecular biological acids. Macromolecular Rapid Communications, Ahead of Print.

Liu, S., Hu, N., Yamamoto, G., Cai, Y., Zhang, Y., Liu, Y., Li, Y., Hashida, T. and Fukunaga, H., 2011, Investigation on CNT/alumina interface properties using molecular mechanics simulations. Carbon, 49(11): 3701-3704.

Liu, Y., Li, R., Peng, H. and Han, Z., 2012, Preparation and damping properties of cupsstacked carbon nanotubes(CSCNTs)/Prime 20. Advanced Materials Research (Durnten-Zurich, Switzerland), 557-559: 493-496.

Liu, Y., Huang, J., Yang, B., Sumpter, B. and Qiao, R., 2014, Duality of the interfacial thermal conductance in graphene-based nanocomposites. Carbon, 75: 169-177.

Liu, Y., Li, J., Li, F., Li, W., Yang, H., Zhang, X., Liu, Y. and Ma, J., 2016, A facile preparation of $\mathrm{CoFe}_{2} \mathrm{O}_{4}$ nanoparticles on polyaniline-functionalised carbon nanotubes as enhanced catalysts for the oxygen evolution reaction. Journal of Materials Chemistry A, 4(12): 4472-4478.

Loh, K. J., Lynch, J. P., Shim, B. S. and Kotov, N. A., 2008, Tailoring piezoresistive sensitivity of multilayer carbon nanotube composite strain sensors. Journal of Intelligent Material Systems and Structures, 19:747-764.

Lu, X., Dou, H., Yang, S., Hao, L., Zhang, L., Shen, L., Zhang, F. and Zhang, X., 2011, Fabrication and electrochemical capacitance of hierarchical graphene/polyaniline/carbon nanotube ternary composite film. Electrochimica Acta, 56(25): 9224-9232.

Lundberg, B. and Sundqvist, B., 1986, Resistivity of a composite conducting polymer as a function of temperature, pressure, and environment: Applications as a pressure and gas concentration transducer. Journal of Applied Physics, 60(3): 1074-1079. 
Ma, C. C., Chuang, K. C. and Pan, S. Y., 2011, Polyvinylidene fluoride film sensors in collocated feedback structural control: application for suppressing impactinduced disturbances. IEEE transactions on ultrasonics, ferroelectrics, and frequency control, 58: 2539-54.

Malte, H., Wichmann, G., Buschhorn, S. T., Böger, L., Adelung, R. and Schulte, K., 2008, Direction sensitive bending sensors based on multi-wall carbon nanotube/epoxy nanocomposites. Nanotechnology, 19 (47): 475503/1$475503 / 5$.

Masuda, H., Tanaka, K. and Sato, T., 1997, A study on passive-adaptive vibration control elements using dilatant materials. Nippon Kikai Gakkai Ronbunshu, Chen, 63: 4122-4127.

McLachlan, D. S., Blaszkiewicz, M. and Newnham, R. E., 1990, Electrical resistivity of composites. Journal of the American Ceramic Society, 73(8): 2187-2203.

Meincke, O., Kaempfer, D., Weickmann, H., Friedrich, C., Vathauer, M. and Warth, H., 2004, Mechanical properties and electrical conductivity of carbon-nanotube filled polyamide- 6 and its blends with acrylonitrile/butadiene/styrene. Polymer, 45(3): 739-748.

Mottaghitalab, V., Xi, B., Spinks, G. M. and Wallace, G. G., 2006, Polyaniline fibres containing single walled carbon nanotubes: Enhanced performance artificial muscles. Synthetic Metals, 156 (11-13): 796-803.

Nakamura, R., Imanishi, J., Imamura, H. and Naribayashi, I., 2004, Polyester compositions containing certain plasticizers with excellent recyclability and biodegradability. US patent: US20150232660.

Odden, D., and Spencer, A., (1996). Phonology: theory and description. Oxford \& Cambridge, Mass. Blackwell Publishers. 13(3): 446-449.

Oh, I. G., Oh, J. H., Jung, J. H., Ju, W. H. and Kim, W. H., 2016, Composition for vibration damping material including polyurethane and graphene-carbon nanotube structure. Korea patent: KR 2016029237.

Okamoto, Y., and W. Brenner, 1964, Polymers: in Organic Semiconductors, Reinhold.

Park, C. H. and Baz, A., 1999, Vibration control of bending modes of plates using active constrained layer damping. Journal of Sound and Vibration, 227(4): 711-734.

Park, M., Kim, H. and Youngblood, J. P., 2008, Strain-dependent electrical resistance of multi-walled carbon nanotube/polymer composite films. Nanotechnology, 19 (5): 55705/1-55705/6. 
Paulitsch, C., Gardonio, P. and Elliott, S. J., 2006, Active vibration control using an inertial actuator with internal damping. The Journal of the Acoustical Society of America, 119: 2131-40.

Pramanik, P. K., Khastgir, D., De, S. K. and Saha, T. N., 1990, Pressure-sensitive electrically conductive nitrile rubber composites filled with particulate carbon black and short carbon fibre. Journal of Materials Science, 25(9): 3848-3853.

Qia, H. J., Teob, K. B. K., Lauc, K. K. S., Boycea, M. C., Milneb, W. I., Robertsonb, J. and Gleasonc, K.K., 2003, Determination of mechanical properties of carbon nanotubes and vertically aligned carbon nanotube forests using nanoindentation, Journal of the Mechanics and Physics of Solids, 51: 2213 - 2237.

Qiao, Y., Li, C. M., Bao, S. J. and Bao, Q. L., 2007, Carbon nanotube/polyaniline composite as anode material for microbial fuel cells. Journal of Power Sources, 170(1): 79-84.

Qu, Y., Dai, K., Zhao, J., Zheng, G., Liu, C., Chen, J. and Shen, C., 2014, The strainsensing behaviors of carbon black/polypropylene and carbon nanotubes/polypropylene conductive composites prepared by the vacuumassisted hot compression. Colloid and Polymer Science, 292: 945-951.

Rao, S.S., 2004, Mechanical vibrations: Pearson-Prentice Hall, Fourth Edition. pg. 150 and pp. 686-687.

Ramaratnam, A. and Jalili, N., 2006, Reinforcement of piezoelectric polymers with carbon nanotubes: pathway to next-generation sensors. Journal of Intelligent Material Systems and Structures, 17 (3): 199-208.

Ramaratnam, A., Jalili, N. and Rajoria, H., 2004, Development of a novel strain sensor using nanotube-based materials with applications to structural vibration control. Proceedings of SPIE-The International Society for Optical Engineering, 5503: $478-485$.

Ramesh, T. C. and Ganesan, N., 1994, Finite element analysis of cylindrical shells with a constrained viscoelastic layer. Journal of Sound and Vibration, 172 (3): 359370.

Renninger J., 2004, Understanding damping techniques for noise and vibration control. http://foamsealant.com.au/understanding-damping-techniques-for-noise-andvibration-control/.

Rein, M. D., Breuer, O. and Wagner, H. D., 2011, Sensors and sensitivity: carbon nanotube buckypaper films as strain sensing devices. Composites Science and Technology, 71 (3): 373-381. 
Rijn, C. V., Vogelaar, L., Nijdam, W., Barsema, J. and Wessling, M., 2004, Method of making a product with a micro or nano sized structure and product, US Patent: US $20040028875 \mathrm{~A} 1$.

Roylance, D., 2008, Mechanical properties of materials. MIT.

Saini, P., Choudhary, V., Singh, B. P., Mathur, R. B. and Dhawan, S. K., 2009, Polyaniline-MWCNT nanocomposites for microwave absorption and EMI shielding. Materials Chemistry and Physics, 113 (2-3): 919-926.

Sasikumar, K., Manoj, N. R., Ramesh, R. and Mukundan, T., 2012, Carbon nanotubepolyurethane nanocomposites for structural vibration damping. International Journal of Nanotechnology, 9:1061-1071.

Shui, X. P. and Chung, D. D. L., 1996, A piezoresistive carbon filament polymer-matrix composite strain sensor. Smart Materials and Structures, 5 (2): 243-246.

Shimamura, Y., Kageyama, K., Tohgo, K. and Fujii, T., 2011, Cyclic behavior of electrical resistance type low stiffness, large strain sensor by using carbon nanofiber/flexible epoxy composite. Key Engineering Materials, 462-463: $1200-1205$.

Simotwo, S. K., DelRe, C. and Kalra, V., 2016, Supercapacitor electrodes based on high-purity electrospun polyaniline and polyaniline-carbon nanotube nanofibers. ACS Applied Materials \& Interfaces, 8: 21261-21269.

Sivakkumar, S. R., Kim, W. J., Choi, J. A., MacFarlane, D. R., Forsyth, M. and Kim, D. W., 2007, Electrochemical performance of polyaniline nanofibres and polyaniline/multi-walled carbon nanotube composite as an electrode material for aqueous redox supercapacitors. Journal of Power Sources, 171 (2): 10621068 .

Sobha, A. P. and Narayanankutty, S. K., 2015, Improved strain sensing property of functionalised multiwalled carbon nanotube/polyaniline composites in TPU matrix. Sensors and Actuators A: Physical, 233: 98-107.

Sreekala, R., Muthumani, K., Lakshmanan, N., Gopalakrishnan, N., Sathishkumar, K., Reddy, G. R. and Parulekar, Y. M., 2010, A study on the suitability of NiTi wires for passive seismic response control. Journal of Advanced Materials, 42: 65-76.

Stanway, A., Rademaker, M. and Newman, P., 2004, Healing of severe ulcerative necrobiosis lipoidica with cyclosporin. Australasian Journal of Dermatology, 45 (2): 119-122.

Stanway, R., Sproston, J. L. and El-Wahed, A. K., 1996, Adaptive vibration control using an electrorheological squeeze-flow damper: 110-120. 
Stanway, E. R., Bunker, A. J. and McMahon, R. G., 2003, Lyman break galaxies and the star formation rate of the Universe at $\mathrm{z} \approx 6$. Monthly Notices of the Royal Astronomical Society, 342 (2): 439-445.

Stegmaier, P., 2014, Supporting and vibration damping component and production method. Germany patent: DE 102013009523.

Suhr, J., Koratkar, N., Keblinski, P. and Ajayan, P., 2005, Viscoelasticity in carbon nanotube composites. Nat Mater, 4 (2): 134-137.

Suhr, J., Zhang, W., Ajayan, P. M. and Koratkar, N. A., 2006, Temperature-activated interfacial friction damping in carbon nanotube polymer composites. Nano Letters, 6: 219-223.

Suzuki, K., Yataka, K., Okumiya, Y., Sakakibara, S., Sako, K., Mimura, H. and Inoue, Y., 2016, Rapid-response, widely stretchable sensor of aligned MWCNT/elastomer composites for human motion detection. ACS Sensors, 1: $817-825$.

Tasis, D., Tagmatarchis, N., Bianco, A. and Prato, M., 2006, Chemistry of carbon nanotubes. Chemical Reviews, 106 (3): 1105-1136.

Taya, M., Kim, W. J. and Ono, K., 1994, Electro-mechanical coupling behavior of conductive short fiber reinforced elastomers. AMD, 193: 61-72.

Tehrani, M., Safdari, M., Boroujeni, A. Y., Razavi, Z., Case, S, Dahmen, K., Garmestani, H. and Al-Haik, M. S., 2013, Hybrid carbon fiber/carbon nanotube composites for structural damping applications. Nanotechnology, 24:155704/1-155704/11.

Tian, S., Cui, F. and Wang, X., 2008, New type of piezo-damping epoxy-matrix composites with multi-walled carbon nanotubes and lead zirconate titanate. Materials Letters, 62 (23): 3859-3861.

Tian, S. and Wang, X., 2008, Fabrication and performances of epoxy/multi-walled carbon nanotubes/piezoelectric ceramic composites as rigid piezo-damping materials. Journal of Materials Science, 43: 4979-4987.

Toker, M., 2014, A high performance solid polyurethane elastomer seismic isolator with or without an elastomer core or lead core. Patent: WO 2014104995.

Tombler, T. W., Zhou, C., Alexseyev, L., Kong, J., Dai, H., Liu, L., Jayanthi, C. S., Tang, M. and Wu, S. Y., 2000, Reversible electromechanical characteristics of carbon nanotubes under local-probe manipulation. Nature, 405 (6788): 769-772.

Torvik, P. J., 2009, Coatings for enhanced passive damping. Ceramic Engineering and Science Proceedings, 29: 3-18. 
Torquato, S., Hyun, S. and Donev, A., 2003, Optimal design of manufacturable threedimensional composites with multifunctional characteristics. Journal of Applied Physics, 94 (9): 5748-5755.

Tran, V. C., Nguyen, V. H., Nguyen, T. T., Lee, J. H., Huynh, D. C. and Shim, J. J., 2016, Polyaniline and multi-walled carbon nanotube-intercalated graphene aerogel and its electrochemical properties. Synthetic Metals, 215: 150-157.

Trindade, M. A. and Maio, C. E. B., 2008, Multimodal passive vibration control of sandwich beams with shunted shear piezoelectric materials. Smart Materials and Structures, 17(5): 055015/1-055015/10.Takahashi, H., Arisaka, H. and Saegusa, T., 2016, Shaker and active vibration control device. Japanese patent: JP2016151298 A 20160822.

Uchino, K, 2000, Ferroelectric Devices. New York, Marcel Dekker.

Ungar, E. E. and Kerwin, E. M., 1962, Loss factors of viscoelastic systems in terms of energy concepts. The Journal of the Acoustical Society of America, 34 (7): 954957.

Valentová, H. and Stejskal, J., 2010, Mechanical properties of polyaniline, Synthetic Metals, 160: 832-834.

Varadan, V. K., Subramanian, H. and Varadan, V. V., 1998, Wireless remote microaccelerometer. Proceedings of SPIE-The International Society for Optical Engineering, 3316: 497-503.

Wang, C. Y., Mottaghitalab, V., Too, C. O., Spinks, G. M. and Wallace, G. G., 2009, Polyaniline and polyaniline-CNT composite fibres as battery materials in ionic liquid electrolyte. in Electrospun Nanofibers Nanotubes Res. 1-19.

Wang, L., Maruf, S. H., Maniglio, D. and Ding, Y., 2012, Fabrication and characterizations of crosslinked porous polymer films with varying chemical compositions. Polymer, 53: 3749-3755.

Wang, X., Yong, Z. Z., Li, Q. W., Bradford, P. D., Liu, W., Tucker, D. S., Cai, W., Wang, H., Yuan, F. G. and Zhu, Y. T., 2013, Ultrastrong, stiff and multifunctional carbon nanotube composites. Materials Research Letters, 1: 19-25.

Wang, X., Wang, X. and Zou, W., 2016, Preparation and photocatalytic property of polyaniline/carbon nanotubes composite. Guisuanyan Xuebao, 44: 71-74.

Whitby, R. L. D., Fukuda, T., Maekawa, T., James, S. L. and Mikhalovsky, S. V., 2008, Geometric control and tuneable pore size distribution of buckypaper and buckydiscs. Carbon, 46 (6): 949-956. 
Wichmann, M. H. G., Buschhorn, S. T., Gehrmann, J. and Schulte, K., 2009, Piezoresistive response of epoxy composites with carbon nanoparticles under tensile load. Physical Review B: Condensed Matter and Materials Physics, 80: 245437/1-245437/8.

Xia, H., 1993, Fiber-enhanced viscoelastic damping polymers and their application to passive vibration control. $125 \mathrm{pp}$.

Xie, Z., Peng, B., Zhang, J., Zhang, X., Yue, Z. and Li, L., 2015, Highly (100)-oriented $\mathrm{Bi}\left(\mathrm{Ni}_{1 / 2} \mathrm{Hf}_{1} / 2\right) \mathrm{O}_{3}-\mathrm{PbTiO}_{3}$ relaxor-ferroelectric films for integrated piezoelectric energy harvesting and storage system. Journal of the American Ceramic Society, 98: 2968-2971.

Xie, Z., He, Z., Feng, X., Xu, W., Cui, X., Zhang, J., Yan, C., Carreon, M. A., Liu, Z. and Wang, Y., 2016, Hierarchical sandwich-like structure of ultrafine N-rich porous carbon nanospheres grown on graphene sheets as superior lithium-ion battery anodes. ACS Applied Materials \& Interfaces, 8: 10324-10333.

Xiong, J., Zheng, Z., Qin, X., Li, M., Li, H. and Wang, X., 2006, The thermal and mechanical properties of a polyurethane/multi-walled carbon nanotube composite. Carbon, 44: 2701-2707.

Xu, W. and Allen, M. G., 2013, Deformable strain sensors based on patterned MWCNTs/polydimethylsiloxane composites. Journal of Polymer Science Part B: Polymer Physics, 51 (20): 1505-1512.

Xu, W., Xie, Z., Cui, X., Zhao, K., Zhang, L., Dietrich, G., Dooley, K. M. and Wang, Y., 2015, Hierarchical graphene-encapsulated hollow $\mathrm{SnO}_{2} \mathrm{SnS}_{2}$ nanostructures with enhanced lithium storage capability. ACS Applied Materials \& Interfaces, 7: 22533-22541.

Yan, T., Li, C., Li, C., Sun, J., Hu, F., Zhou, C., Chen, Y. and Yan, C., 2016, Manufacture method of polyurethane vibration damper containing nano filler.Chinese patent: CN 105693968 A 20160622.

Yin, G., Hu, N., Karube, Y., Liu, Y., Li, Y. and Fukunaga, H., 2011, A carbon nanotube/polymer strain sensor with linear and anti-symmetric piezoresistivity. Journal of Composite Materials, 45: 1315-1323.

Yoshikawa, S., Ota, T., Newnham, R. and Amin, A., 1990, Piezoresistivity in polymerceramic composites. Journal of the American Ceramic Society, 73 (2): 263-267.

Zhan, Y. H., Patel, R., Lavorgna, M., Piscitelli, F., Khan, A., Xia, H. S., Benkreira, H. and Coates, P., 2010, Processing of polyurethane/carbon nanotubes composites using novel minimixer. Plastics, Rubber and Composites, 39: 400-410. 
Zhang, W., Suhr, J. and Koratkar, N., 2006, Carbon nanotube/polycarbonate composites as multifunctional strain sensors. Journal of Nanoscience and Nanotechnology, 6: $960-964$.

Zhao, C., Yin, Z. and Wang, J., 2015, Efficient electrochemical conversion of $\mathrm{CO}_{2}$ to $\mathrm{HCOOH}$ using Pd-polyaniline/CNT nanohybrids prepared in situ. Chem. Electro. Chem, 2 (12): 1974-1982.

Zhao, M. H., Wang, Z. L. and Mao, S. X., 2004, Piezoelectric characterization of individual zinc oxide nanobelt probed by piezoresponse force microscope. Nano Letters, 4 (4): 587-590.

Zheng, Q., Ma, Z. and Gong, S., 2016, Multi-stimuli-responsive self-healing metallosupramolecular polymer nanocomposites. Journal of Materials Chemistry A: Materials for Energy and Sustainability, 4: 3324-3334.

Zhong, X., Bailey, N. A., Schesing, K. B., Bian, S., Campos, L. M. and Braunschweig, A. B., 2013, Materials for the preparation of polymer pen lithography tip arrays and a comparison of their printing properties. Journal of Polymer Science, Part A: Polymer Chemistry, 51: 1533-1539.

Zhou, X., Shin, E., Wang, K. W. and Bakis, C. E., 2004, Interfacial damping characteristics of carbon nanotube-based composites. Developments in carbon nanotube and nanofibre reinforced polymers, 64 (15): 2425-2437. 
VITA

WEIWEI LIN

\section{EDUCATION}

2007

B. S. Polymer Materials Science and Engineering

Excellent Student Scholarship. 2003-2007.

Ningbo Institute of Technology, Zhejiang University

Ningbo, Zhejiang, China

2010

M. S. Materials Physics and Chemistry

Zhejiang University of Technology

Hangzhou, Zhejiang, China

2016

Doctoral Candidate in Materials Engineering

Teaching Assistant 2010 - 2011

Research Assistant 2011 - 2016

Dissertation Year Fellowship, Summer and Fall Semester, 2016

Society for Optics and Photonics (SPIE), Student Member 2014-present

Materials Research Society (MRS), Student Member 2014-present

Florida International University

Miami, Florida, United States

\section{PUBLICATIONS}

1. Lin, W., Rotenberg, Y., Ward, K., Fekrmandi, H. and Levy, C., "Polyaniline/MultiWalled Carbon Nanotube Composites for Structural Vibration Damping and Strain Sensing", Journal of Materials Research, 1-11, 2016.

2. Lin, W., Rotenberg, Y., Ward, K., Fekrmandi, H. and Levy, C., "Multifunctional Materials of Polyurethane/Buckypaper for Structural Vibration Damping and Strain Sensing”, IJIRSET, 4 (12): 12531-12544, 2015.

3. Lin, W., Kong, X. and Levy, C., "Preparation of Polyaniline/Multiwall Carbon Nanotubes Nanocomposites”, Sensors \& Transducers, 187 (4): 129-137, 2015.

4. Feng, J., Zhong, M. and Lin, W., "Fabrication of Polyethylene Surface with Stable Superhydrophobicity by Nanoparticle Assisted Thermal Micromolding Process", Journal of nanoscience and nanotechnology, 12 (3): 2679-2684, 2012. 
5. Lin, W., Zhong, M. and Feng, J., "Characteristics and Methods of Superhydrophobic Surface Anticoagulation Property”, JCRTER, 13 (51): 1011310116, 2009.

6. Feng, J., Lin, W., Zheng, J., Zhong, M. and Huang, B., "Method for preparing superhydrophobic surface by using nano-particles for assisting micromolding", Chinese Patent No. CN 101537682 A, 2009.

7. Zheng, J., Lin, W. and Feng, J., "Fabricating TiO2/PU composite superhydrophobic film by micro-molding”, Biomaterials congress, 2009, Tianjing, China.

8. Lin, W., Rotenberg, Y., Ward, K., Fekrmandi, H. and Levy, C., "Preparation of Polyurethane/Buckypaper composites films and characterization of their Structural Vibration Damping and Strain Sensing Properties", 4th International Conference and Exhibition on Materials Science and Engineering, 2015, Orlando, USA.

9. Lin, W. and Levy, C., "Preparation of Buckypaper/DYAD/Polyanilline/Multiwall Carbon Nanotubes and Characterization of Their Damping Properties", BIT's 5th Annual World Congress of Nano Science \& Technology, 2015, Xi'an, China.

10. Lin, W. and Levy, C., "Synthesizing of Polyaniline/Multiwall Carbon Nanotubes Composites and Characterization of Their Damping and Strain Sensing Properties", GPSC's annual Scholarly Forum, 2016, FIU. 